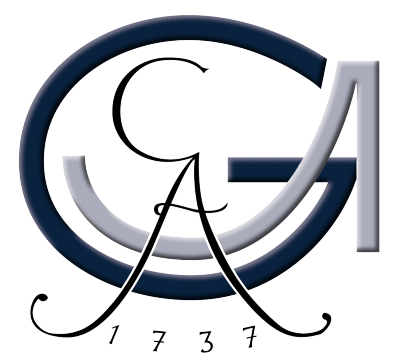

\title{
Influence of lons on the Assembly of Vimentin Intermediate Filaments
}

\author{
Dissertation \\ for the award of the degree \\ "doctor rerum naturalium" \\ of the Georg-August-Universität Göttingen
}

within the doctoral program

Göttingen Graduate School of Neuroscience, Biophysics and Molecular Bioscience (GGNB) of the Georg-August-University School of Science (GAUSS)

\author{
submitted by \\ Manuela Denz \\ from Augsburg (Germany)
}

Göttingen, 2019 



\title{
Members of the Thesis Committee:
}

\author{
Prof. Dr. Sarah Köster
}

Institute for X-Ray Physics

Georg-August-Universität, Göttingen

Prof. Ph.D. Thomas Burg

Department for Biological Micro- and Nanotechnology

Max Planck Institute for Biophysical Chemistry, Göttingen

\section{Prof. Dr. Ralf Ficner}

Institute for Microbiology and Genetics

Georg-August-Universität, Göttingen 



\section{Members of the Examination Board:}

Referee: Prof. Dr. Sarah Köster

Institute for X-Ray Physics

Georg-August-Universität, Göttingen

$2^{\text {nd }}$ Referee: Prof. Ph.D. Thomas Burg

Department for Biological Micro- and Nanotechnology

Max Planck Institute for Biophysical Chemistry, Göttingen

\section{Further members of the Examination Board:}

\section{Prof. Dr. Ralf Ficner}

Institute for Microbiology and Genetics

Georg-August-Universität, Göttingen

Prof. Dr. Tim Salditt

Institute for X-Ray Physics

Georg-August-Universität, Göttingen

Prof. Dr. Andreas Janshoff

Institute for Physical Chemistry

Georg-August-Universität, Göttingen

\section{Prof Dr. Helmut Grubmüller}

Department of Theoretical and Computational Biophysics

Max Planck Institute for Biophysical Chemistry, Göttingen

Date of Oral Examination:

10.02.2020 


\section{Contents}

$\begin{array}{lll}\text { List of Abbreviations } & \text { XI }\end{array}$

List of Figures $\quad$ XIII

List of Tables $\quad$ XVII

1 Introduction 1

References . . . . . . . . . . . . . . . . . . . . . . . 3

2 State of the Art and Theoretical Background 5

2.1 Intermediate Filaments . . . . . . . . . . . . . . . . . . . . . . . . 5

2.1.1 The Cytoskeleton . . . . . . . . . . . . . . . . . . . . . . . . 5

2.1.2 Architecture of Intermediate Filaments . . . . . . . . . . . . . . . 6

2.1.3 Assembly of Intermediate Filaments . . . . . . . . . . . . . . . . 7

2.2 Microscopy . . . . . . . . . . . . . . . . . . . . . . . . . . . . . 9

2.2.1 Fluorescence Microscopy. . . . . . . . . . . . . . . . . . . . . 9

2.2.2 Atomic Force Microscopy. . . . . . . . . . . . . . . . . . . . . 11

2.3 Small Angle X-Ray Scattering . . . . . . . . . . . . . . . . . . . . . . 13

2.3.1 General Principles of SAXS . . . . . . . . . . . . . . . . . . . . 13

2.3.2 SAXS Instruments. . . . . . . . . . . . . . . . . . . . . . . . 19

2.3.3 Measurement Principle and Data Treatment . . . . . . . . . . . . . 20

2.3.4 Data Analysis . . . . . . . . . . . . . . . . . . . . . . . . . 21

2.3.5 Applications of SAXS . . . . . . . . . . . . . . . . . . . . . . 23

2.4 Microfluidics . . . . . . . . . . . . . . . . . . . . . . . . . . . . 24

2.4.1 Flow at Low Reynolds Number . . . . . . . . . . . . . . . . . . 24

2.4.2 Mixing in Microfluidic Devices . . . . . . . . . . . . . . . . . . 25

2.4.3 Microfluidic Devices for Diffusive Mixing . . . . . . . . . . . . . . 26

References . . . . . . . . . . . . . . . . . . . . . . 28 
$3 \quad$ Materials and Methods $\quad 35$

3.1 Protein Reconstitution . . . . . . . . . . . . . . . . . . . . . . . . 35

3.2 Protein Assembly . . . . . . . . . . . . . . . . . . . . . . . . . . 36

3.2.1 SAXS Experiments . . . . . . . . . . . . . . . . . . . . . . . 37

3.2.2 Microscopy Experiments . . . . . . . . . . . . . . . . . . . . 37

3.3 Static SAXS Experiments. . . . . . . . . . . . . . . . . . . . . . . . 38

3.3.1 In-house SAXS Setup . . . . . . . . . . . . . . . . . . . . . . 38

3.3.2 Calibration Measurements . . . . . . . . . . . . . . . . . . . . 39

3.3.3 Measurements . . . . . . . . . . . . . . . . . . . . . . . . . 40

3.3.4 Data Processing . . . . . . . . . . . . . . . . . . . . . . . . 41

3.4 Microscopy Experiments . . . . . . . . . . . . . . . . . . . . . . . 43

3.4.1 Atomic Force Microscopy. . . . . . . . . . . . . . . . . . . . . 44

3.4.2 Fluorescence Microscopy. . . . . . . . . . . . . . . . . . . . . 44

3.5 Time-Resolved SAXS Experiments . . . . . . . . . . . . . . . . . . . . 44

3.5.1 Finite Element Method Simulations . . . . . . . . . . . . . . . . 44

3.5.2 Preparation of Microfluidic Channels . . . . . . . . . . . . . . . . 46

3.5.3 Experimental Setup at ESRF - ID13 Beamline. . . . . . . . . . . . . 50

3.5.4 Experimental Setup at SLS - cSAXS Beamline. . . . . . . . . . . . . 50

3.5.5 Sample Preparation and Measurement at the Beamline . . . . . . . . 51

3.5.6 Data Processing . . . . . . . . . . . . . . . . . . . . . . . 53

3.6 Materials . . . . . . . . . . . . . . . . . . . . . . . . 55

References . . . . . . . . . . . . . . . . . . . . . 57

4 Comparison of Different Buffers and their Effects on Intermediate Filaments 61

4.1 Importance of Buffers . . . . . . . . . . . . . . . . . . . . . . . . . 61

4.2 Influence of the Buffers on Tetrameric Protein . . . . . . . . . . . . . . . 63

4.3 Influence of the Buffers on Filamental Protein . . . . . . . . . . . . . . . 66

4.4 Summary and Discussion . . . . . . . . . . . . . . . . . . . . . . . 68

References . . . . . . . . . . . . . . . . . . . 73

$5 \quad$ The Influence of Ions on the Assembly of Intermediate Filaments 77

5.1 The Role of Ions for Intermediate Filaments ． . . . . . . . . . . . . . . 77

5.2 Vimentin Filaments Assembled in the Presence of Monovalent Ions . . . . . . 79

5.3 Vimentin Filaments Assembled in the Presence of Divalent Ions ． . . . . . . 85

5.4 Vimentin Filaments Assembled in the Presence of Hexammine-cobalt(III)

Chloride . . . . . . . . . . . . . . . . . . . . . . . . . . . . . 91

5.5 Vimentin Filaments Assembled in the Presence of Spermine . . . . . . . . . 95 
5.6 Summary and Discussion . . . . . . . . . . . . . . . . . . . . . . . 99

References . . . . . . . . . . . . . . . . . . . . . . 109

6 Cyclic olefin copolymer as an X-ray compatible material for microfluidic devices 113

6.1 Abstract . . . . . . . . . . . . . . . . . . . . . . . . . . . . . 114

6.2 Introduction . . . . . . . . . . . . . . . . . . . . . . . . . . 114

6.3 Materials and Methods . . . . . . . . . . . . . . . . . . . . . . . . 116

6.3.1 Device Fabrication and Usage . . . . . . . . . . . . . . . . . . . 116

6.3.2 Protein Purification and Assembly . . . . . . . . . . . . . . . . . 118

6.3.3 Small Angle X-Ray Scattering . . . . . . . . . . . . . . . . . . . 120

6.3.4 Data Analysis . . . . . . . . . . . . . . . . . . . . . . . . . 121

6.3.5 Flow Simulations . . . . . . . . . . . . . . . . . . . . . . . . 122

6.4 Results and Discussion . . . . . . . . . . . . . . . . . . . . . . . . 123

6.4.1 Device Characterization . . . . . . . . . . . . . . . . . . . . . 123

6.4.2 Protein in Flow . . . . . . . . . . . . . . . . . . . . . . . . . 125

6.5 Conclusions. . . . . . . . . . . . . . . . . . . . . . . . . . . 125

6.6 Acknowledgments . . . . . . . . . . . . . . . . . . . . . . . . . . 127

6.7 Supplementary Information . . . . . . . . . . . . . . . . . . . . . . 128

References . . . . . . . . . . . . . . . . . . . . . . 133

7 Summary, Discussion and Outlook 139

References . . . . . . . . . . . . . . . . . . . . . . 144

$\begin{array}{lll}\text { A Calculation of the Correction Factor } & 147\end{array}$

A.1 Calculating the Correction Factor . . . . . . . . . . . . . . . . . . . . 147

References . . . . . . . . . . . . . . . . . . . . . 150

B Model Fits $\quad 151$

B.1 Model Fits of Vimentin Assembled in the Presence of $\mathrm{KCl}$. . . . . . . . . . 152

B.2 Model Fits of Vimentin Assembled in the Presence of $\mathrm{NaCl}$. . . . . . . . . . 156

B.3 Model Fits of Vimentin Assembled in the Presence of $\mathrm{MgCl}_{2}$. . . . . . . . . . 160

B.4 Model Fits of Vimentin Assembled in the Presence of $\mathrm{CaCl}_{2}$. . . . . . . . . . 164

B.5 Model Fits of Vimentin Assembled in the Presence of Hexammine-cobalt(III)

chloride . . . . . . . . . . . . . . . . . . . . . . . . . . . . . . 168

B.6 Model Fits of Vimentin Assembled in the Presence of Spermine . . . . . . . . 173

$\begin{array}{ll}\text { List of Publications } & 177\end{array}$

$\begin{array}{ll}\text { Acknowledgement } & 179\end{array}$ 


\section{List of Abbreviations}

\begin{tabular}{ll}
\hline Abbreviation & Full name \\
\hline AFM & atomic force microscopy \\
Be-CRL & beryllium compound refractive lenses \\
BG & background \\
CF & correction factor \\
COC & cyclic olefin copolymer \\
DTT & 1,4-di-thiothreitol \\
EDTA & ethylenediaminetetraaceti acid \\
EGTA & ethylene glycol-bis(2-aminoethylether)-N, N,N',N'-tetraacetic acid \\
EM & electron microscopy \\
FEM & finite element method \\
F(q) & form factor \\
HMDS & hexamethyldisilazane \\
IF & intermediate filament \\
MAC & methylamine hydrochloride \\
MF & microfilament \\
MOPS & 3-(N-morpholino)propanesulfonic acid \\
MT & mircotubles \\
MW & molecular weight \\
NA & numerical aperture \\
PB & phosphate buffer \\
PBS & phosphate-buffered saline \\
pddf & pair distance distribution function \\
PDMS & polydimethylsiloxane \\
Pe & Péclet number \\
PVC & polyvinylchloride \\
Re & Reynolds number \\
SAXS & small ange X-ray scattering \\
$S(q)$ & structure factor \\
TRIS & tris(hydroxymethyl)aminomethan \\
ULF & unit-length-filament \\
USAXS & ultra small angle X-ray scattering \\
WAXS & wide angle X-ray scattering \\
wt & wildtype \\
\hline & \\
\hline
\end{tabular}




\section{List of Figures}

2.1 Fluorescence images of cytoskeletal filaments. . . . . . . . . . . . . 5

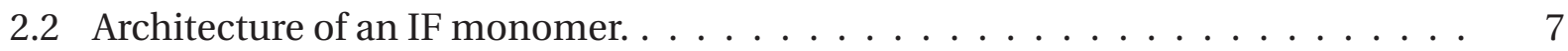

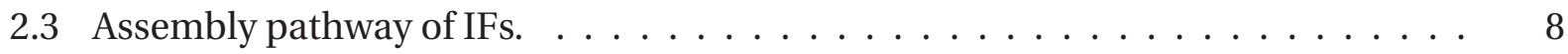

2.4 The principle of fluorescence microscopy. . . . . . . . . . . . . . . 10

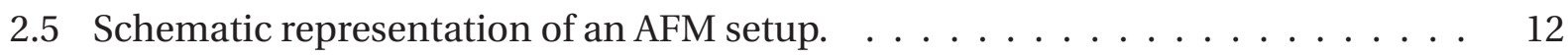

2.6 Schematic representation of the SAXS principle. . . . . . . . . . . . 13

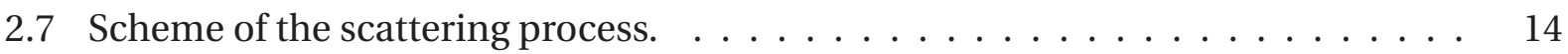

2.8 Schematic representation of the form factor and structure factor. $\ldots \ldots \ldots$

2.9 Calculated form factors of different shaped objects. . . . . . . . . . . . . . 18

2.10 Example of polydispersity. . . . . . . . . . . . . . . . . . . . . 19

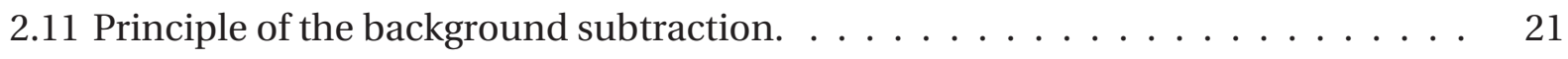

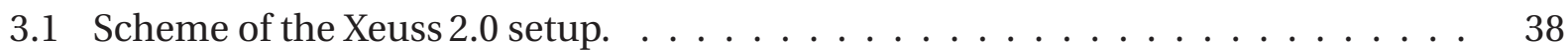

3.2 Scattering patterns of the calibration measurements. . . . . . . . . . . . 40

3.3 Variance of capillary diameters. . . . . . . . . . . . . . . . 41

3.4 Description of the model used for fitting the data. . . . . . . . . . . . . 43

3.5 Device geometry and protein stream width. $\ldots \ldots \ldots \ldots \ldots$

3.6 Manufacturing steps of the device master. . . . . . . . . . . . . . . . 46 
3.7 Manufacturing of UV-curable adhesive/Kapton devices. . . . . . . . . . . . . . . 49

3.8 Scheme of the experimental hutch II of the ID13 beamline at the ESRF $\ldots \ldots \ldots$

3.9 Representation of the cSAXS beamline at the SLS . . . . . . . . . . . . . 51

3.10 Sample holder for the microfluidic devices. . . . . . . . . . . . . . . 52

3.11 Schematic representation of the mesh used in synchrotron experiments. . . . . . 53

4.1 Typical AFM images of tetrameric vimentin in different buffers. . . . . . . . . 63

4.2 Scattering profiles of vimentin filaments assembled with $100 \mathrm{mM} \mathrm{KCl}$ in $2 \mathrm{mM}$ MOPs buffer. . . . . . . . . . . . . . . . . . . . . . . . . . 64

4.3 Scattering profiles of tetrameric vimentin at low (2 $\mathrm{mM})$ and high (20 $\mathrm{mM})$ buffer concentration. . . . . . . . . . . . . . . . . . . .

4.4 Guinier analysis of the scattering profiles of vimentin in the the three buffers (TRIS, PB and MOPS) at low (2 mM) and high (20 mM) concentration. . . . . . . .

4.5 AFM images of vimentin assembled to filaments in $100 \mathrm{mM} \mathrm{KCl}$ in different buffers. 67

4.6 Scattering profiles of vimentin filaments at low $(2 \mathrm{mM})$ and high $(20 \mathrm{mM})$ buffer

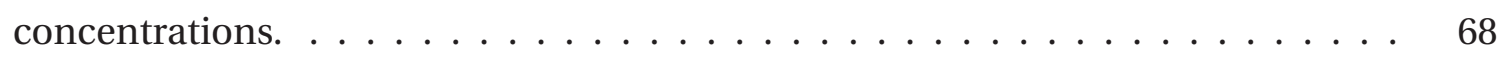

4.7 Guinier analysis of the scattering profiles of vimentin filaments in the low ( $2 \mathrm{mM})$ and high $(20 \mathrm{mM})$ concentrations of the three buffers (TRIS, PB and MOPS). . . 68

4.8 Comparison of vimentin tetramers in $\mathrm{PB}$ at $\mathrm{pH} 7.5$ and $8.4 \ldots \ldots \ldots \ldots$

4.9 Force strain curves for vimentin filaments. . . . . . . . . . . . . . . . 71

5.1 Scheme of a vimentin monomer and the corresponding amino acids sequence of vimentin. . . . . . . . . . . . . . . . . . . . . 78

5.2 Scattering profiles of vimentin assembled with monovalent ions at various ion

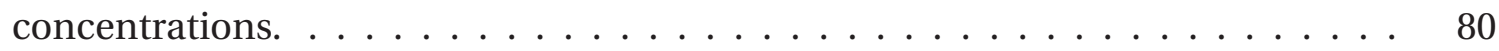

5.3 Guinier analysis of the scattering profiles for vimentin filaments assembled with

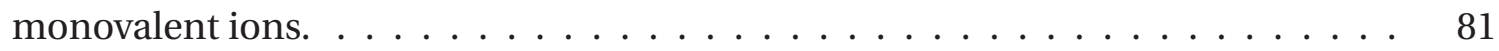

5.4 Scattering profiles of vimentin assembled with $20 \mathrm{mM} \mathrm{NaCl}$ and the respective fit. 83 
5.5 Analysis of vimentin assembled with $\mathrm{KCl}$ or $\mathrm{NaCl}$ at different concentrations using a model for micelles. . . . . . . . . . . . . . . . . . . . . . . . . . .

5.6 Fraction of tetramers in the measured solution for vimentin protein assembled with monovalent ions. . . . . . . . . . . . . . . . . . . . . . . . . 84

5.7 Fluoresence microscopy images of vimentin assembled with monovalent ions. .

5.8 Scattering profiles of vimentin assembled with divalent ions at eight different con-

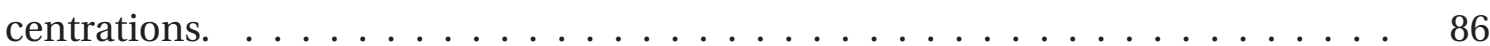

5.9 Guinier analysis of vimetnin filaments assembled with $\mathrm{CaCl}_{2}$ and $\mathrm{MgCl}_{2} \ldots \ldots$. . . 87

5.10 Analysis of vimentin assembled with $\mathrm{CaCl}_{2}$ and $\mathrm{MgCl}_{2}$ using a micelle model. . .

5.11 Fraction of tetramers in the measured solution for vimentin protein assembled with $\mathrm{MgCl}_{2}$ and $\mathrm{CaCl}_{2} \ldots \ldots \ldots \ldots \ldots \ldots \ldots$

5.12 Scattering profiles of vimentin assembled by $4 \mathrm{mM} \mathrm{MgCl}_{2}$ and the respective fit.

5.13 Fluorescence microscopy images of vimentin assembled with divalent ions. . . .

5.14 Scattering profiles of vimentin assembled with the trivalent salt hexamminecobalt(III) chloride in the range of $0.01 \mathrm{mM}$ to $0.5 \mathrm{mM}$. . . . . . . . . . . . . . . .

5.15 Analysis of the scattering profiles of vimentin assembled with the trivalent ion hexammine-cobalt(III) chloride . . . . . . . . . . . . . . . . . .

5.16 Analysis of vimentin assembled with hexammine-cobalt(III) chloride using the model.

5.17 Fraction of tetramers in the measured solution for vimentin protein assembled with hexammine-cobalt(III) chloride. . . . . . . . . . . . . . . . . . . .

5.18 Fluorescence microscopy image of vimentin assembled with $0.05 \mathrm{mM}$ hexammine-cobalt(III) chloride. . . . . . . . . . . . . . . . . . 95

5.19 Scattering profiles of vimentin assembled with spermine. . . . . . . . . . . . 96

5.20 Analysis of the scattering profiles of vimentin assembled with spermine. . . . . .

5.21 Analysis of vimentin assembled with the tetravalent molecule spermine using the model described by Pedersen. 
5.22 The calculated fraction of tetramers in the measured solution for vimentin protein assembled with the tetravalent molecule spermine. . . . . . . . . . . . . . . . 99

5.23 Microscopy image of vimentin assembled with $0.03 \mathrm{mM}$ spermine. . . . . . . . 99

5.24 Model fit to vimentin assembled in the presence of $2 \mathrm{mM} \mathrm{CaCl}_{2} \ldots \ldots \ldots$

5.25 The precipitation threshold observed for vimentin assembled by different ions against the valency of the ions. . . . . . . . . . . . . . . . . 105

5.26 Radius of gyration of the cross-section calculated from the Guinier region in dependence of the ionic strength. . . . . . . . . . . . . . . . 106

$5.27 I(0)$ values against the radius of gyration from Guinier analysis. . . . . . . . . . 107

6.1 Manufacturing steps of a COC device. . . . . . . . . . . . . . . . . . . 117

6.2 Geometry of the microfluidic device. . . . . . . . . . . . . . . . . . 119

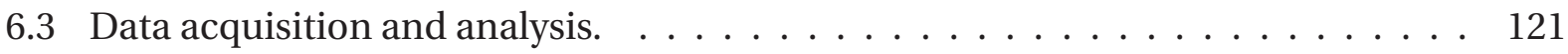

6.4 Colloid scattering curves with corresponding form factor. . . . . . . . . . . . . . 124

6.5 Vimentin measurement in a COC device, performed at the cSAXS beamline, SLS. 126

A.1 Scattering profile of water after background subtraction and correction with the transmission values. . . . . . . . . . . . . . . . . . . . . . . . 148

A.2 Scattering profiles of water (without the sample chamber in vacuum) and glassy

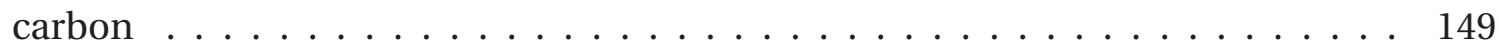




\section{List of Tables}

2.1 Overview of IF classes, IF protein examples and their occurrence . . . . . . . . . 6

3.1 Overview of all used buffers and their respective concentrations and $\mathrm{pH}$ values. . $\quad 36$

3.2 Overview of ions used for assembly experiments in $2 \mathrm{mM}$ MOPS buffer at $\mathrm{pH} 7.5$ and their respective concentrations during SAXS measurements . . . . . . . . 37

3.3 Overview of used ions in $2 \mathrm{mM}$ MOPS buffer at $\mathrm{pH} 7.5$ and their respective concentrations during microscopy measurements . . . . . . . . . . . . 38

3.4 Overview of the different sample to detector distances, the corresponding $q$-range and the respective real space dimensions. . . . . . . . . . . . . . 39

3.5 Used chemicals and the respective company . . . . . . . . . . . . . . . 55

5.1 Results of the Guinier analysis of vimentin assembled with $\mathrm{KCl} / \mathrm{NaCl}$ at different

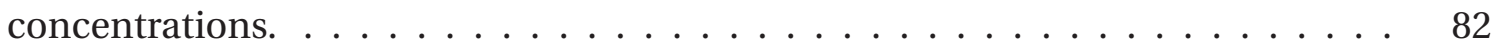

5.2 Results of the Guinier analysis of vimentin assembled with $\mathrm{CaCl}_{2} / \mathrm{MgCl}_{2}$ at different concentrations. . . . . . . . . . . . . . . . . . . .

5.3 Results of the Guinier analysis of vimentin assembled with hexammine-cobalt(III) chloride at different concentrations. . . . . . . . . . . . . . . . . .

5.4 Results of the Guinier analysis of vimentin assembled with the tetravalent spermine at different concentrations. . . . . . . . . . . . . . . . . . . . 


\section{Chapter 1}

\section{Introduction}

The human body consists of many different cell types, which enclose various molecules like DNA, lipids or proteins. All of these molecules acquire a plethora of functions, like structural components in the cell membrane (lipids) or storing the genetic information (DNA). There are many different proteins with a large variety of functions ranging from signal transduction to stabilizing tasks, or carrying out chemical reactions. A protein system that every eukaryotic cell exhibits is the cytoskeleton, which is composed of three different types of protein filaments: microfilaments, microtubules and intermediate filaments. Besides the filaments, numerous crosslinkers and motor proteins are necessary for the correct function of the cytoskeleton. An appropriate interplay of the protein system is necessary for the function of the cytoskeleton. To understand the physical and chemical properties of the system, it is of interest to understand the functions of each underlying protein individually. Thus, in vitro studies are performed on single protein classes like e.g. intermediate filaments. It is not only important to understand the function of the protein itself but also its assembly process. Occasionally protein aggregates are formed instead of the fully functional protein, and the causes as well as the effects need to be understood. For some proteins, like the class of intermediate filaments, ions have an influence on the assembly process [1]. As the cell itself contains a large variety of ions [2], it is important to understand the fundamental influences of different ions on the assembly of intermediate filaments.

In this context, the assembly process of the intermediate filament vimentin is investigated in detail. In vitro, the assembly of vimentin can be initiated by adding monovalent ions [3-5]. In addition, it has been shown that the assembly of vimentin protein into filaments can be initiated by divalent or even trivalent ions. Differences in the filament radius, the homogeneity of the radius of the filaments as well as aggregation threshold were found [3, 6-9]. In all the mentioned studies only one or two different ions were investigated and thus, no complete picture 
of different ions on the assembly process is available. In this thesis, the assembly of vimentin in the presence of six different ions, with varying valencies is investigated and compared in a systematic way. Using small angle X-ray scattering the lateral assembly of the filaments can be detected, meaning the radius of the filament as well as the molecular weight can be retrieved. Using different microscopy techniques (atomic force microscopy and fluorescence microscopy) the elongated filaments can be observed and it can be investigated whether single filaments or networks are formed. The combination of both methods allows for information on the architecture of the assembled filament itself.

Besides static experiments, where only the resulting filament upon addition of the ion is observed, it is important to investigate the temporal and spatial evolution of the assembly process, to get better insights into time scales of the assembly process as well as the structural evolution of the protein. This can be achieved by combining microfluidics with, e.g., small angle X-ray scattering. The assembly is initiated after mixing the assembly reagent with the protein. By following the flow using X-rays, the assembly process can be followed temporally. However, it is crucial for these experiments to have an appropriate microfluidic device which is resistant to $\mathrm{X}$-rays so that data of good quality can be obtained.

In Chapter 2 an overview on the cytoskeleton with special focus on the intermediate filaments is given. The architecture as well as the assembly process are described in more detail. In the second half of the Chapter, the applied methods are introduced. First, different microscopy techniques are briefly introduced, followed by a longer introduction to small angle X-ray scattering, as small angle X-ray scattering is the main method used in the scope of this thesis. Besides a short introduction to the general principle of small angle X-ray scattering, data treatment and analysis will be described. In the last part of the Chapter, an introduction to microfluidics will be given. In Chapter 3 the applied methods and the materials used are described and listed respectively.

The importance of buffers in in vitro studies and comparing the effects of different buffers on the vimentin protein is presented in Chapter 4 . A buffer that has previously not been used for intermediate filament studies, will be compared to two commonly used buffers. The protein is investigated with small angle X-ray scattering as well as atomic force microscopy. In Chapter 5 , the influence of different ions on the assembly of vimentin filaments is investigated. The assembly of vimentin in the presence of six different ions is investigated with small angle X-ray scattering and fluorescence microscopy. The results from Chapter 6 are published as "Cyclic olefin copolymer as an X-ray compatible material for microfluidic devices" [10]. In Chapter 7, the results are summarized and the main conclusions drawn. Finally, a short outlook for future experiments is given. 


\section{References}

1. Block, J., Schroeder, V., Pawelzyk, P., Willenbacher, N. \& Köster, S. Physical properties of cytoplasmic intermediate filaments. Biochim. Biophys. Acta, Mol. Cell Res. 1853, 30533064 (2015).

2. Janmey, P. A., Slochower, D. R., Wang, Y.-H., Wen, Q. \& Cēbers, A. Polyelectrolyte properties of filamentous biopolymers and their consequences in biological fluids. Soft Matter 10, 1439 (2014).

3. Brennich, M. E. et al. Impact of ion valency on the assembly of vimentin studied by quantitative small angle X-ray scattering. Soft Matter 10, 2059-2068 (2014).

4. Mücke, N., Kirmse, R., Wedig, T., Leterrier, J. \& Kreplak, L. Investigation of the morphology of intermediate filaments adsorbed to different solid supports. J. Struct. Biol. 150, 268-276 (2005).

5. Lopez, C. G., Saldanha, O., Huber, K. \& Köster, S. Lateral association and elongation of vimentin intermediate filament proteins: A time-resolved light-scattering study. P. Natl. Acad. Sci. U.S.A. 113, 11152-11157 (2016).

6. Wu, H. et al. Effect of the divalent cations zinc and calcium on the structure and mechanics of reconstituted vimentin intermediate filaments (2019).

7. Dammann, C., Herrmann, H. \& Köster, S. Competitive Counterion Binding Regulates the Aggregation Onset of Vimentin Intermediate Filaments. Isr. J. Chem. 56, 614-621 (2015).

8. Hofmann, I., Herrmann, H. \& Franke, W. W. Assembly and structure of calcium-induced thick vimentin filaments. Eur. J. Cell Biol. 56, 328-341 (1991).

9. Herrmann, H., Häner, M., Brettel, M., Ku, N.-O. \& Aebi, U. Characterization of distinct early assembly units of different intermediate filament proteins. J. Mol. Biol. 286, 1403-1420 (1999).

10. Denz, M. et al. Cyclic olefin copolymer as an X-ray compatible material for microfluidic devices. Lab Chip 18, 171-178 (2018). 


\section{Chapter 2}

\section{State of the Art and Theoretical Background}

\subsection{Intermediate Filaments}

In the following section, a short introduction to intermediate filaments (IFs) will be given. The role of IFs in the cell, their architecture and the assembly process of IFs is described.

\subsubsection{The Cytoskeleton}

The cytoskeleton of eukaryotic cells is a 3D network composed of three main cytoskeletal proteins: microfilaments (MFs, diameter 7-9 nm), microtubules (MTs, diameter $24 \mathrm{~nm}$ ) and intermediate filaments (IFs, diameter $10 \mathrm{~nm}$ ) shown in Fig. 2.1.
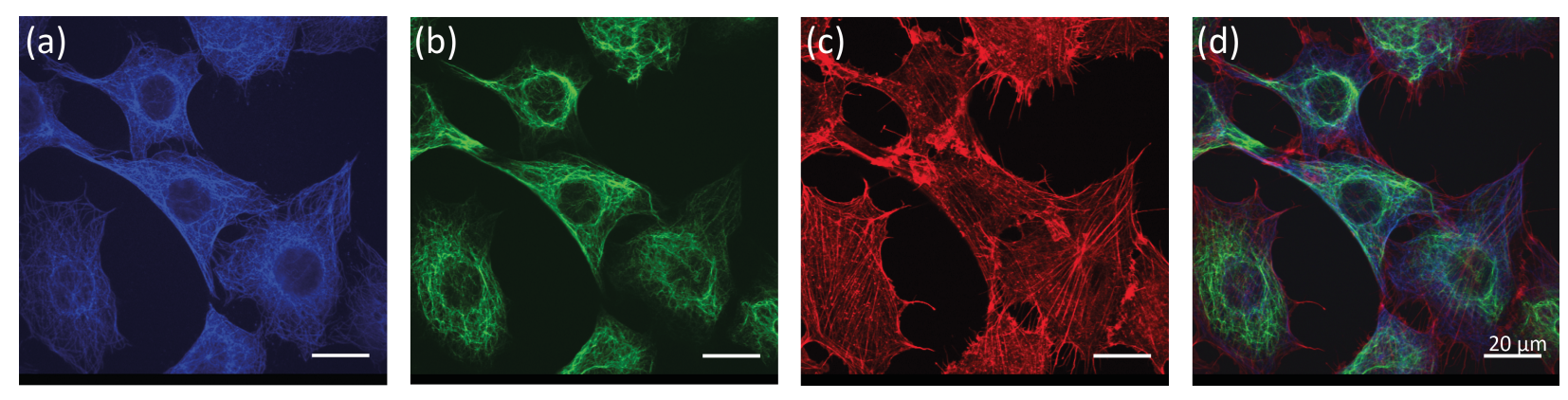

Figure 2.1: Fluorescence images of cytoskeletal filaments. The three different cytoskeletal proteins (a) MTs (b) vimentin (IFs), and (c) actin (MFs) in 3 T3 fibroblast cells. (d) Overlay of all three channels. Note that the contrast in (a) is enhanced for better visualization. Images courtesy of Dr. Ulrike Rölleke.

Among the many functions the cytoskeleton carries out, there are three main tasks. (I) The cytoskeleton organizes the cell contents, (II) it is necessary for cell migration and shape change of the cell, and (III) it connects the cell to the environment [1-3]. Every cell has to be adapted to a certain environment [1]. Especially during cell division, the cytoskeleton undergoes drastic 
changes [4]. Thus, the cytoskeleton is one of the main contributors to the mechanical properties of the cell. In addition, signal transduction and cellular transport is conducted over the cytoskeletal filaments [5-7].

It is the interplay of all filaments which make the cytoskeleton this powerful and determines the mechanical properties of cells [8]. Each filament class has its own unique functions. The polar MT are hollow cylinders comprising 13 protofilaments and they are the most rigid filaments in the cytoskeleton $[9,10]$. They are mainly involved in intracellular transport (of, e.g., proteins, vesicles and organelles) and important for the positioning of organelles in the cell [7, 11]. Furthermore, they play a key role during mitosis as they form the spindle apparatus which divides the chromosomes during the cell division process [3]. MFs are polar helical polymers of actin $[9,10]$. Their main function is to maintain the cell shape and they are furthermore involved in cell signalling and cell migration [7]. Compared to MFs and MTs, IFs are the least well studied filaments. They are more flexible than MFs and MTs and they introduce mechanical stability to the cell, especially when the cell is deformed.

\subsubsection{Architecture of Intermediate Filaments}

In contrast to the conserved MFs or MTs, IFs are highly diverse between different cell types [12]. For example, mesenchymal cells express the IF vimentin, whereas epithelial cells express keratin IFs and neurofilaments are found in neurons [2]. Until now, over 70 different genes for IFs have been found in humans [13], which are divided into five classes regarding their sequence homology $[1,9,12]$.

Table 2.1: Overview of IF classes, IF protein examples and their occurrence. Table adopted from [1, 9]

\begin{tabular}{lll}
\hline Class & Intermediate Filaments & Occurrence \\
\hline I & acidic keratins & epithelia, hair, horn \\
II & basic-neutral keratins & epithelia, hair, horn \\
III & vimentin & mesenchymal cells \\
& desmin & muscle cells \\
& glial fibrillary acidic protein & astrocytes \\
& synemin & muscle cells \\
IV & neurofilaments & neurons \\
& nestin & neuroepithelial stem cells \\
& $\alpha$-internexin & neurons \\
V & lamins & nucleus \\
\hline
\end{tabular}

Even though IFs are highly diverse and have different roles within the cell, they share the same secondary structure. This conserved structure consists of a rod domain flanked by a random coiled head (N-terminus) and a random coiled tail domain (C-terminus). The rod domain of 
IFs consists of an $\alpha$-helix divided into three coiled-coils, namely the coil 1A and 1B, and a coil 2 region, separated by linkers L1 and L12 (Fig. 2.2) [9, 14].

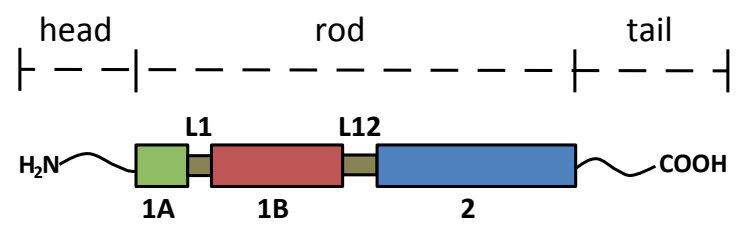

Figure 2.2: Architecture of an IF monomer. The secondary structure consists of a head, rod and tail domain. The head and tail domains are intrinsically disordered, whereas the rod domain consists of an $\alpha$-helix divided in three coiled-coils (coil 1A, coil 1B and coil 2) connected by the linkers L1 and L12.

It has been shown that at the beginning of coil 2, a so called stutter region exist in all IFs studied so far [3]. The normal heptad repeat $(\text { abcde } f g)_{n}$ is interrupted by a hendecad repeat $[14,15]$. The length of the rod domain is conserved throughout all IFs. It is approximately $43 \mathrm{~nm}$ long and shows similar segments of patterns in all IFs, with the exception of the IF lamin, which is slightly longer $[9,14,16]$. The length of the head and tail region however, varies from only a few amino acids to several hundreds of amino acids [3, 17].

\subsubsection{Assembly of Intermediate Filaments}

Another difference between IFs and MF and MT is the assembly process. Whereas the assembly of MF and MT is nucleotide driven and needs additional energy [7], IF assembly is nonnucleotide driven. Furthermore, it follows a hierarchical pathway and the IFs have the possibility to assemble into 8 - $12 \mathrm{~nm}$ wide filaments $[18,19]$. Fig. 2.3 schematically shows the assembly process of vimentin, however, the process can be transferred to all other assembly processes of IFs and is thus kept generic in the following.

Two monomers form a parallel coiled-coil dimer. This dimer can either be a homodimer (dimer of two monomers of the same kind; e.g. vimentin) or a heterodimer (two monomers from different kinds; e.g. keratin). In the case of keratin, the two monomers usually come from different sequence homology classes (class I and II) [20]. However, it has also been shown, that keratin K8 can form homodimers. Yet, the keratin K8 homodimers do not assemble into filaments [21]. Two dimers then form an anti-parallel, half-staggered tetramer. In vitro, this process occurs spontaneously when reducing denaturating agents (e.g. urea). It has been shown that vimentin forms tetramers even at an urea concentration of $5 \mathrm{M}$ [17, 22]. All IF proteins, with the exception of lamins, form tetramers when no denaturing agents (e.g. urea) are present [3].

Many tetramers then laterally assemble to form so called unit-length-filaments (ULFs), which have a length of roughly $58 \mathrm{~nm}$ [17]. The number of tetramers within an ULF depends on the 


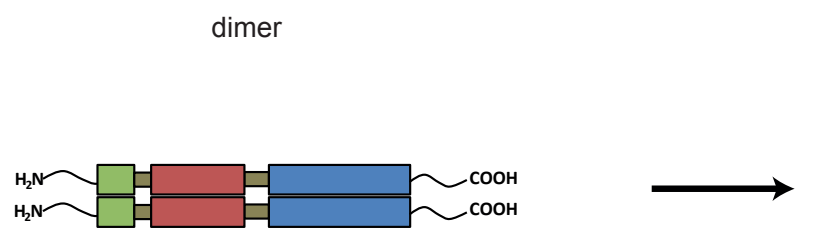

filament

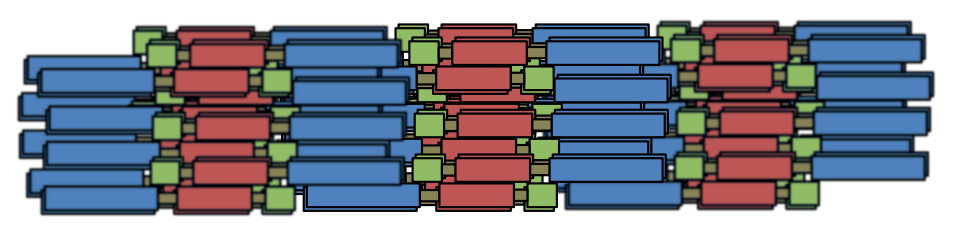

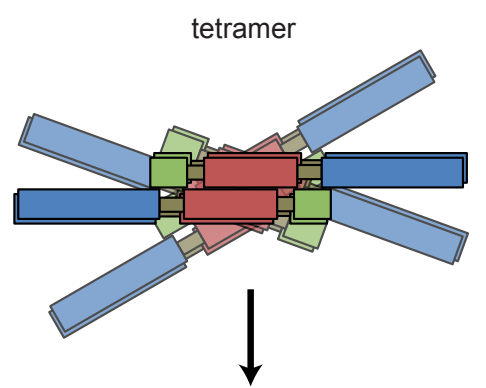

unit-length filament (ULF)

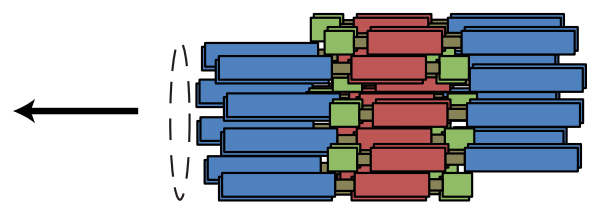

Figure 2.3: Assembly pathway of IFs: First two monomers assemble into a parallel coiled-coil dimer (homo- or heterodimer). Two dimers then assemble in an anti-parallel half staggered way to form tetramers. Several tetramers assemble laterally to form ULFs. The number of tetramers per ULF depends on the IF. ULFS then longitudinal anneal to from filaments. For some IFs a final compaction step is performed.

IF. For example vimentin forms ULFs with an average of 32 monomers, whereas keratin forms ULFs with approximately 16 subunits [23-25]. These numbers are only average numbers, as it was shown that the number of subunits does not only change between different IF types, but also between the same IF type and even within one filament [11, 17, 26].

ULFs then anneal longitudinally to form $\mu \mathrm{m}$ long filaments. Some IFs (e.g. vimentin, desmin) undergo an additional compaction step, to form mature filaments [27]. The persistence length of IFs ranges from 0.3 - $2 \mu \mathrm{m}$ depending on the IF [28, 29]. In vitro, vimentin assembly can be performed by adding monovalent ions or by changing the $\mathrm{pH}$ and the ionic strength of the buffer. The assembly of keratin is, for example, initiated by decreasing the $\mathrm{pH}$ and increasing the ionic strength of the buffer [21, 25, 30]. For vimentin, it is sufficient to add $\mathrm{KCl}$ to trigger the assembly $[24,31,32]$.

To assemble the IF protein, two different methods can be utilized. A slow assembly can be performed, where the assembly buffer is brought to the protein via dialysis. This procedure leads to relatively homogeneous thick filaments. Another way of assembling the protein is via the kickstart method, where the assembly buffer and the protein are mixed instantaneously [33]. This method results in less homogeneous filaments, with a larger variance in the filament diameter [16]. It is speculated, that the inhomogeneity in the filament diameter comes from the varying subunit number in the ULF $[11,26]$.

In the fully formed filament, the tails of the monomers protrude from the filament [3, 20, 34]. It 
was shown that the tails are not necessary for assembly [35], however they seem to play a role in controlling the filament width [17]. As the tails are protruding from the filament, they are also important in network formation [3]. On the contrary, it has been shown that the head domain, positioned in the filament core, is essential for assembly [12]. By truncating the head domain of several IFs, it was shown that those were not able to form filaments on their own [12].

\subsection{Microscopy}

In this study, two different microscopy techniques are used to visualize vimentin filaments, namely fluorescence microscopy and atomic force microscopy (AFM). A brief introduction to fluorescence microscopy and AFM will be given.

To visualize and observe all kinds of samples, different methods and techniques can be used. A very simple way is using a light microscope. It is not clear who invented the first light microscope, however one potential inventor is Zacharias Janssen who invented the microscope in the 1590s [36]. In the late $17^{\text {th }}$ century Antoni van Leeuwenhoek developed a single lens microscope, with which he was able to observe bacteria [36]. Since then, microscopes have constantly been improved and nowadays, many different techniques (for example: phase contrast, fluorescence, bright field) can be used. Even with all these improvements the optical resolution, that expresses the minimum distance between two object points necessary to separate them, is limited by:

$$
d=\frac{1.22 \lambda}{2 \mathrm{NA}}
$$

where $d$ is the distance between two objects, $\lambda$ is the wavelength and NA is the numerical aperture. The resolution can be increased by using superresolution techniques, electron microscopy (EM), or AFM. With the mentioned techniques distances down to a few nm can be resolved.

\subsubsection{Fluorescence Microscopy}

The main literature used for this Subchapter are [37, 38]. Fluorescence is based on the principle of an excited electron returning to the ground state, by which the electron emits the absorbed energy in the form of a photon. The pathway for an electron to decay into the ground state after excitation is shown in the Jablonski diagram (Fig. 2.4a) [39].

If a molecule, for example a fluorophore is excited by a photon, absorption takes place, meaning that an electron leaves the ground state $\left(S_{0}\right)$ for the excited state $\left(S_{1}\right)$. The absorption process is very fast on the timescales of $10^{-15} \mathrm{~s}$. Both the ground state as well as the excited state have several vibrational levels. If the excited electron is in a higher vibrational level in the $S_{1}$ state, it will 
(a)

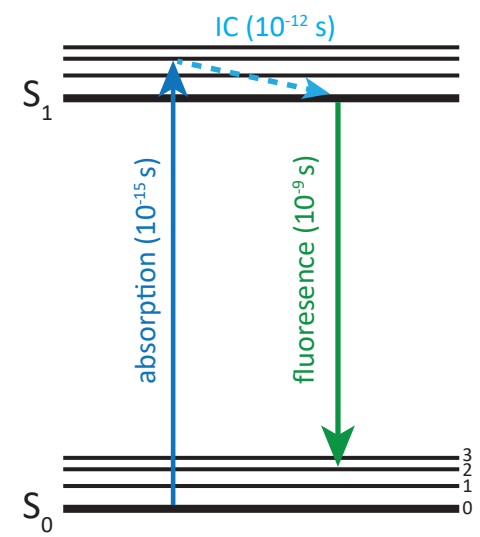

(b)

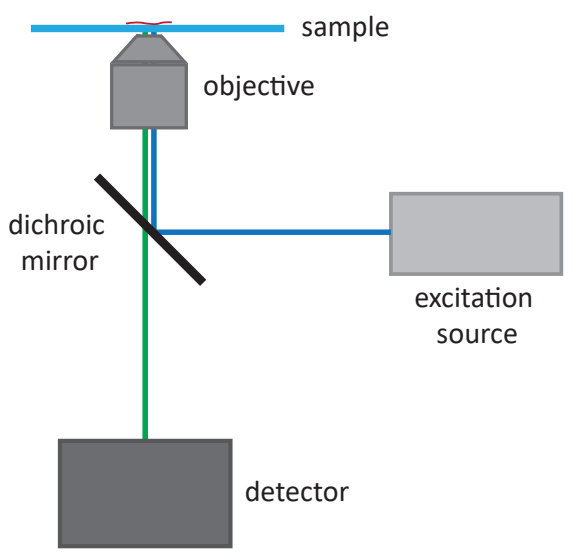

Figure 2.4: The principle of fluorescence microscopy. (a) The Jablonski diagram. If a molecule is excited a photon is absorbed and the excited state usually at a higher vibrational energy level is reached. By losing energy in a non-radiative manner (IC), the lowest level of the excited state is reached. Now fluorescence can occur where the molecule relaxes into the ground state. (b) A schematic representation of a fluorescence microscope. Light (blue) from an excitation source is reflected by a dichroic mirror (black bar). After the light passes the objective the sample is illuminated. The sample emits light at a longer wavelength as explained in (a). The emitted light (here shown in green) passes through the dichroic mirror and the emitted light is then recorded by a detector.

first relax to the lowest vibrational level in $S_{1}$ via internal conversion (IC). IC usually happens on timescales of $10^{-12} \mathrm{~s}$ and results in the loss of energy. If the electron then returns to the $\mathrm{S}_{0}$ state this can happen via fluorescence, which is on the order of $10^{-9} \mathrm{~s}$. Due to the reduction of energy through the IC, the returned molecule has a longer wavelength than when it was excited. This shift was first observed by Sir G. G. Stokes and is called the Stokes shift. The emission spectra, is not only shifted to a longer wavelength but is also the mirrored absorption spectra.

A fluorophore is characterized by its quantum yield and the fluorescence lifetime. The quantum yield $(\Phi)$ is described by:

$$
\Phi=\frac{\text { number of emitted photons }}{\text { number of absorbed photons }} .
$$

In an ideal case the quantum yield would be one, however, due to the internal conversion and the resulting loss of energy, the quantum yield is always lower than one.

The fluorescence lifetime is the average time the molecule spends in the excited state before returning to the ground state. The fluorescence lifetime can be written as:

$$
\tau_{f l}=\frac{1}{\text { number of emitted photons }} .
$$

This principle is applied in fluorescence microscopy. A schematic overview on the principle of a 
fluorescence microscope is shown in Fig. 2.4b. Light from an excitation source (e.g. laser-light) is reflected by a dichroic mirror. A dichroic mirror is a mirror which reflects a certain wavelength while the remaining light spectrum will pass through it. The reflected light is focused with an objective on the sample and excites the fluorophores, which are normally bound to the system under investigation. As described above, the photon is absorbed and the emitted fluorescence spectra has a longer wavelength than the excitation spectrum. The emitted light passes through the objective and the dichroic mirror and is recorded on a detector. A drawback of fluorescence microscopy is that the sample under investigation needs to be labeled. Bulky fluorophores, which might be bigger than the protein itself, can influence the activity of the protein under investigation [40]. Furthermore, the resolution is limited by the used wavelength (see Equation 2.1).

\subsubsection{Atomic Force Microscopy}

A different way to image a sample is by using AFM. As the name already indicates, AFM measures forces which emerge between the sample and the probe while the sample is scanned and thus, the resolution is not limited by Equation 2.1 but rather by the probe used during measurements. The forces between the sample and the probe can be described with the Lennard-Jones potential [41]:

$$
V_{i}=E_{0}\left[\left(\frac{r_{0}}{r_{i}}\right)^{12}-2\left(\frac{r_{0}}{r_{i}}\right)^{6}\right]
$$

where $E_{0}$ describes the binding energy, $r_{0}$ denotes to the equilibrium distance of the LennardJones potential and $r_{i}$ is the distance between the probe and the $i^{\text {th }}$ atom. The Lennard-Jones potential describes the interaction between uncharged and unbound atoms. While at large distances between the atoms, the attractive forces like Van-der-Waals dominate, the repulsive forces (Pauli repulsion) increase if the distance between the atoms is reduced.

To perform an AFM measurement, five components are necessary [42]. (I) First of all, a hard probe (tip) on a soft cantilever is needed to scan the sample. (II) A system which detects the deflection of the cantilever, (III) as well as a feedback system to monitor the deflection is required. (IV) A high precision scanning stage is needed to move the sample so it can be rasterized. (V) Finally, a system is required which converts the measured deflection into an image. In Fig. 2.5, the basic principle of an AFM measurement is visualized.

The probe itself is made out of a hard material like diamond so it will not deform during the experiments [42]. The cantilever on the other hand, has a soft spring constant and a high resonance frequency. The forces between the sample and the probe causes the cantilever to deflect 


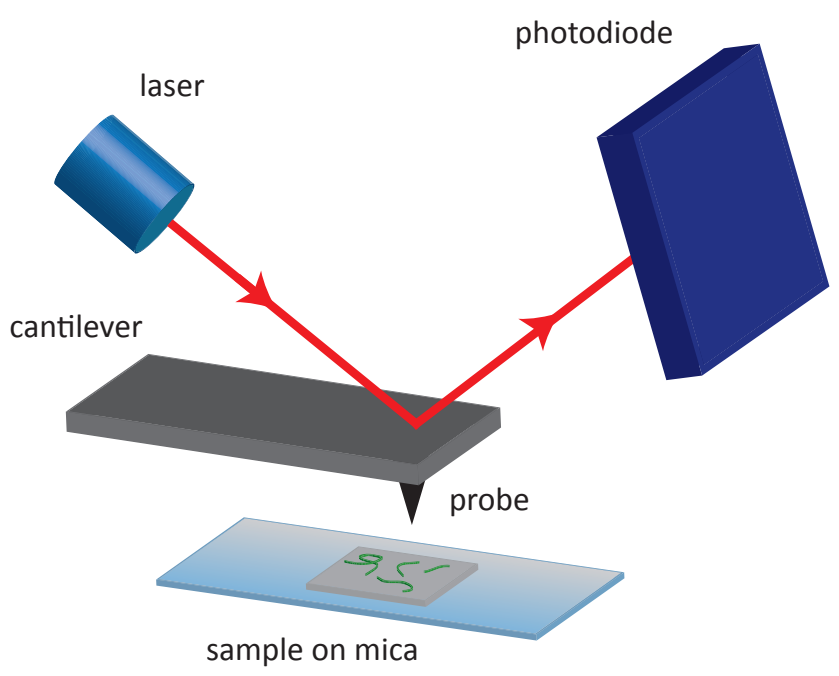

Figure 2.5: Schematic representation of an AFM setup. The sample on a mica slice is scanned by a sharp probe attached to the cantilever. A laser is focused on the tip of the cantilever and the deflection of the cantilever is recorded with a photodiode.

according to Hooks law [43]:

$$
F=-k x
$$

where $F$ is the applied force to the cantilever, $k$ the spring constant of the cantilever and $x$ the displacement of the cantilever upon interaction. To detect the deflection of the cantilever a laser is used. The laser is focused on the tip of the cantilever and the reflected laser signal is recorded. If the cantilever bends upon contact with the sample, the angle of the recorded laser light changes. The change in the angle can then be transformed into an image $[42,44]$.

For imaging the sample two different measuring modes can be applied [43, 44]. In the contact mode, the sample and the probe are in contact at all times and the distance between the sample and the probe never changes, thus the probe is pressed against the sample with a constant force. This is achieved by adapting the $z$-position of the sample stage using Piezo-regulators. A drawback however is, that the sample and the probe can get damaged through the contact. Furthermore, in the case of soft samples, the probe can move the sample while measuring in contact mode. The second measuring mode is the tapping mode, where the cantilever oscillates near its resonant frequency and only "taps" the sample. This measuring mode is more suitable for imaging experiments. By just "tapping" the sample, less artifacts e.g. moving of the sample can be introduced [43-45]. Like fluorescence microscopy, also AFM has a drawback in the sample preparation. Samples need to be brought on special surfaces which can introduce artifacts or change the mechanical properties of the sample $[28,46]$. 


\subsection{Small Angle X-Ray Scattering}

In this study, small angle X-ray scattering (SAXS) is used as the main method to investigate the effect of buffers or ions on the assembly of vimentin IFs. Furthermore, SAXS is used in microfluidic studies to observe the assembly process of IFs. In the following section, an introduction to SAXS is given, followed by a description of data treatment and ways to analyze SAXS data.

\subsubsection{General Principles of SAXS}

Another way to improve the resolution is to use X-rays. An advantage of many X-ray techniques is that no labeling or slicing of the sample is needed, however the measuring technique is quite different to light microscopy. The wavelength of X-rays is approximately 0.1 to $10 \AA$ [47]. In SAXS experiments, length scales from 1-100 nm can be observed, however, with ultra small angle Xray scattering (USAXS) or/and wide angle X-ray scattering (WAXS), the limits on both sides can be extended. The first SAXS measurements were performed in the 1930s by André Guinier and Otto Kratky and the first biological SAXS experiments were performed in the 1950s on proteins like hemoglobin [48]. At that time, the data analysis was limited. Nowadays, thanks to further developments of the technique, data interpretation and analyzing tools, the structure (size and shape), the molecular weight, the cross-section and the surface of macromolecules can be investigated [48-50]. Another advantage of SAXS is that the samples under investigation can be liquid or solid. Fig. 2.6 shows a typical SAXS setup. The X-rays scatter at the sample and the scattered signal is recorded on a detector.

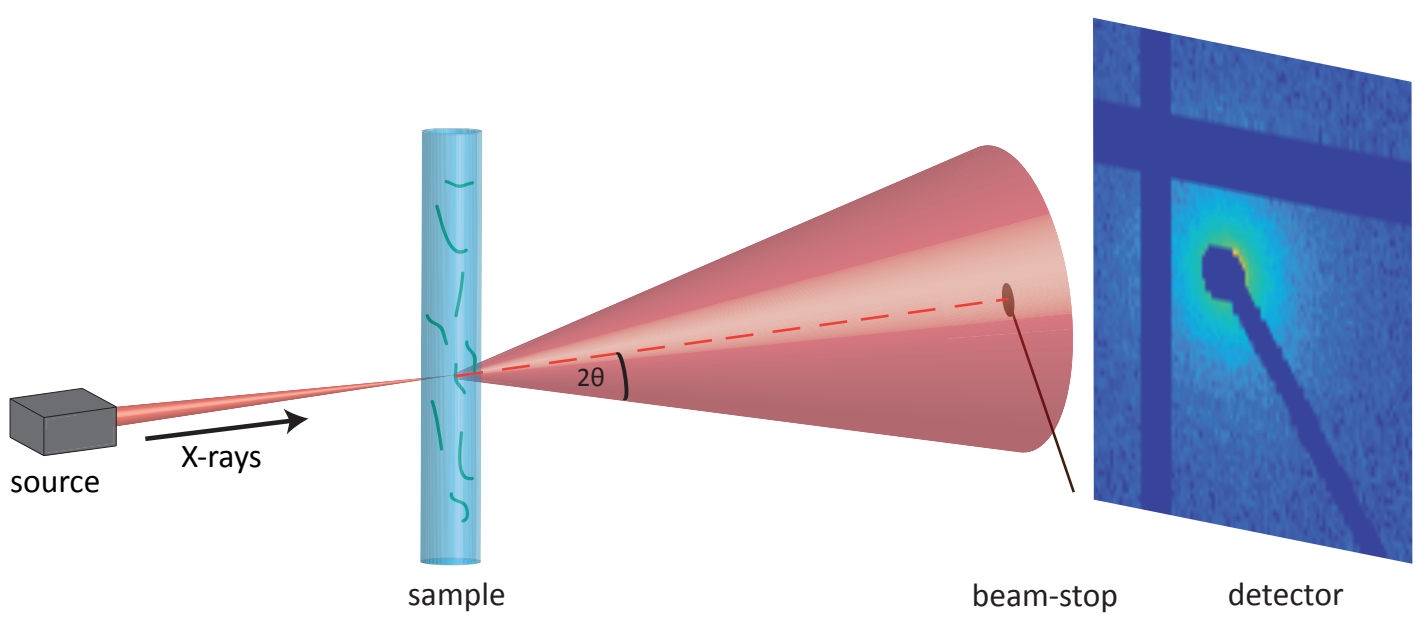

Figure 2.6: Schematic representation of the SAXS principle. X-rays are produced in the source and focused. If the $X$-rays hit the sample, the $X$-rays scatter at the electrons of the particles. The primary beam intensity is blocked by a beam-stop and the scattered signal is recorded on a detector. 
$\mathrm{X}$-rays can be described as electromagnetic waves. As soon as they hit the sample, the X-rays can be scattered by the electrons within the excited volume and the signal is recorded at some distance on a detector. The distance between the sample and the detector can be correlated to the measured angles and defines which length scales are observed. The farther the detector is away from the sample, the smaller the angles which can be recorded and thus, the larger the detected length scales in real space. Usually, the primary beam is blocked by a beam-stop, as the incident beam would damage the detector. The size of the beam-stop limits the lowest achievable $q$-value. However, due to recent detector development it is possible for the primary beam to be detected without damaging the detector and giving access to lower $q$-values [48]; e.g the Xeuss 3.0 from Xenocs (Sassenage, France) is a SAXS machine without a beam-stop. As every particle in the excited volume can contribute to the scattering, SAXS is a bulk technique, but due to the small beam size, it only needs small amounts of sample (several tenth of $\mu \mathrm{L}$ ).

\section{The Scattering Process}

X-rays have an electromagnetic origin and plane wave properties [48]. The electric $(\vec{E})$ and magnetic $(\vec{H})$ fields are perpendicular to each other [51]. By irradiating a sample, a part of the X-rays will pass through the sample. A fraction will be absorbed and transformed in other types of energy (e.g. fluorescence, heat or radiation) and a third part will be scattered (Fig. 2.7a) [51, 52]. Scattering can occur in two different ways; either with the loss of energy (inelastic scattering) or without losing energy (elastic scattering) [51].

(a)

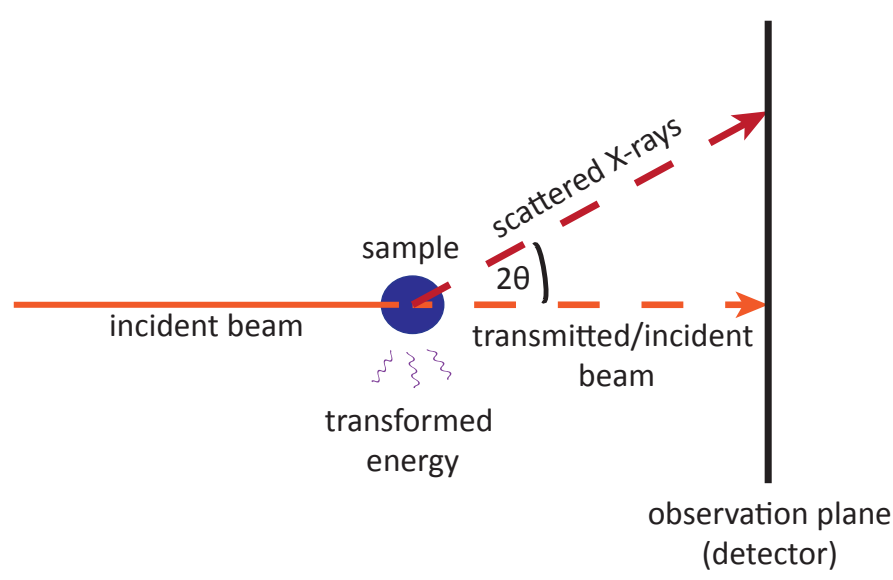

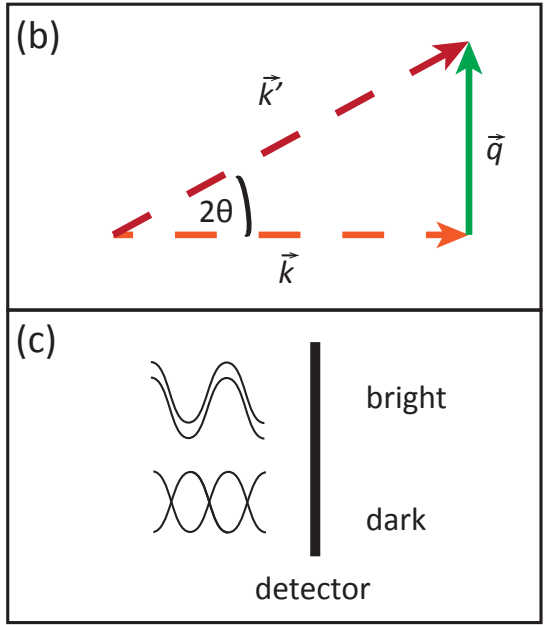

Figure 2.7: Scheme of the scattering process. (a) The incident X-ray beam scatters at the sample. The X-rays can be absorbed and the energy can be transformed into, e.g, heat, or the X-rays can be transmitted or scattered by the sample. The signal is detected on an observation plane (detector). (b) Vector diagram of the scattering process, where $\vec{k}$ denotes the incoming beam and $\vec{k}^{\prime}$ to the scattered X-rays. (c) Sketch of incoming waves. If the waves are in phase a bright spot is visible on the detector. If the waves are out of phase, there will be a dark spot on the detector. Images are based on [49, 52, 53]. 
Compton scattering is a form of inelastic scattering. It is produced when a photon hits an electron and a part of the photon energy is transferred to the electron [51]. Due to the energy transfer, the scattered radiation has a different wavelength and therefore does not result in any interference. The scattered signal does not hold any structural information and can be neglected as in SAXS experiments only small angles are investigated [50].

Rayleigh scattering is an example of elastic scattering of X-rays at small particles. [51, 52]. In this case, the photon does not transfer any energy if it collides with an electron. The electron starts to oscillate with the same frequency as the incoming X-rays and the electron emits radiation with the same frequency, however the direction of the scattered radiation is different. The emitted radiation produces scattering which carries the object structural information.

The efficiency with which X-rays are scattered is expressed as the scattering cross-section $(\sigma)$ [54].

$$
\sigma=\frac{\text { scattered energy }}{\text { incident energy per unit area }}
$$

By multiplying the scattering cross-section of one particle by the particle density, the macroscopic scattering cross-section can be defined. With solution SAXS measurements, mostly macromolecules are investigated. In this case, it is not possible to measure discrete atoms or electrons as the molecules are too large, and the signal of an individual atom is smeared out [54].

When X-rays interact with matter, not only one but many electrons start to oscillate and the emitted waves, which are synchronized with the incoming plane waves, produce interference patterns at the detector. If two waves are in phase, the interference is constructive and a bright spot on the detector is visible. If two waves are out of phase, they cancel each other out and a dark spot is visible on the detector (Fig. 2.7c). If waves are only partly in phase, a less bright spot on the detector is visible. By collecting this information from all pixels on the detector, a 2D scattering pattern can be recorded. The scattering pattern provides information about the orientation and the distances of atoms within the illuminated area. The distances are expressed by the momentum transfer or scattering vector " $\vec{q}$ ", which is the difference between the wave vectors from the scattered wave $\vec{k}^{\prime}$ and the incident wave $\vec{k}$ (Fig. 2.7b)

$$
\vec{q}=\vec{k}^{\prime}-\vec{k}
$$

The momentum transfer can also be expressed using the scattering angle $2 \theta$ (angle between $\vec{k}$ and $\left.\vec{k}^{\prime}\right)$ :

$$
q=\frac{4 \pi}{\lambda} \sin (\theta)
$$


where $\lambda$ is the wavelength of the radiation. As the scattering is measured in reciprocal space, the real space distance $D$ corresponding to $q$ is $D=2 \pi / q$ [55] and, therefore, $q$ has the dimension $\mathrm{nm}^{-1}$. By performing an azimuthal integration, the 2D scattering pattern can be transformed into a $1 \mathrm{D}$ scattering profile, where the scattering intensity $I(q)$ is plotted against $q$.

In general the measured scattering intensity for randomly oriented, centrosymmetric and identical particles can be described as:

$$
I(q)=F(q) \cdot S(q)
$$

where $F(q)$ is the form factor and $S(q)$ the structure factor $[55,56]$.

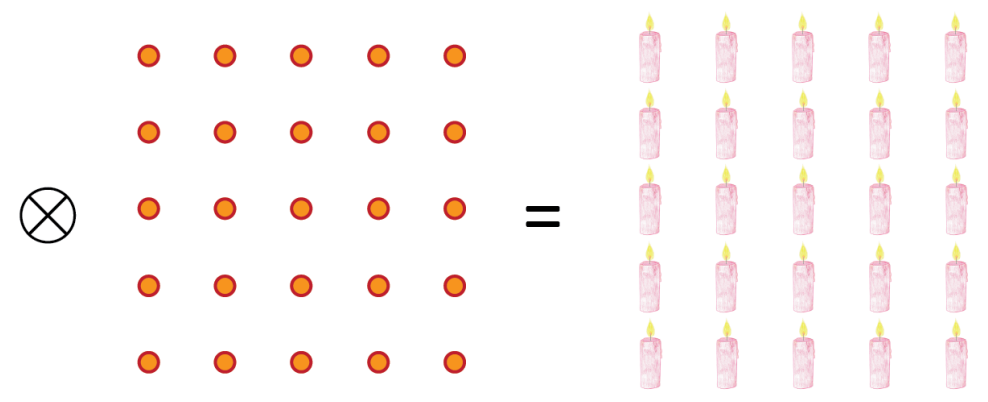

Figure 2.8: Schematic representation of the form factor and structure factor. The form factor is represented by the image (candle) on the left hand side. The lattice (orange circles) describes the structure factor. By convolution of the form factor and the structure factor, the crystal structure is produced.

Fig. 2.8 schematically describes the form factor and the structure factor. If both a form factor as well as a structure factor is present, the measured intensity corresponds to the convolution of both and represents the crystal structure.

\section{The Form Factor}

$F(q)$ describes the scattering due to the electrons of a single particle. A general description of the form factor can be written as:

$$
F(q)=\int \rho(\vec{r}) e^{(i \vec{q} \vec{r})} \mathrm{d} \vec{r}
$$

where $\rho(\vec{r})$ is the density of the particle. If the sample is very diluted so that particles are far away from each other and do not interact, the form factor alone is sufficient to describe the scattering profile while the structure factor (which is related to interparticle interactions) is negligible.

Lets consider a solid sphere with a radius $R$ and a density $\rho(\vec{r})=\rho$. Furthermore, the volume $\mathrm{d} V$ is defined as $\mathrm{d} V=r^{2} \sin \theta \mathrm{d} \theta \mathrm{d} \phi \mathrm{d} r$. The particle has spherical symmetry so that $\vec{q}=\left(0,0, q_{z}\right)$. Writing the scalar product $\vec{q} \vec{R}=q R \cos \theta$, the form factor of a sphere can be rewritten as: 


$$
F(q)=\rho \int_{0}^{R} \int_{0}^{\pi} \int_{0}^{2 \pi} e^{-i q r \cos \theta} r^{2} \sin \theta \mathrm{d} \theta \mathrm{d} \phi \mathrm{d} r .
$$

The integrals are solved one after each other and the steps are shown in the following. First an integration in respect to $\phi$ yields:

$$
F(q)=2 \pi \rho \int_{0}^{R} \int_{0}^{\pi}(\cos (q r \cos (\theta))+i \sin (q r \cos (\theta))) r^{2} \sin \theta \mathrm{d} \theta \mathrm{d} r
$$

Using a variable substitution with $u(q, r, \theta)=q r \cos (\theta)$ and $\mathrm{d} u(q, r, \theta)=-q r \sin (\theta)$ the equation above can be rewritten as:

$$
F(q)=2 \pi \rho \int_{0}^{R} \int_{0}^{\pi}(\cos (u(q, r, \theta))+i \sin (u(q, r, \theta))) r^{2} \frac{-\mathrm{d} u(q, r, \theta)}{q r} \mathrm{~d} \theta \mathrm{d} r
$$

Integrating with respect to $\theta$ yields:

$$
F(q)=2 \pi \rho \int_{0}^{R} 2 \frac{r}{q} \sin (q r) \mathrm{d} r
$$

Finally an integration in respect to $r$ yields:

$$
F(q)=\frac{4 \pi}{q} \rho\left(\frac{\sin (q R)-q R \cos (q R)}{q^{2}}\right)
$$

Rewriting the form factor

$$
F(q)=4 \pi \rho R^{3}\left(\frac{\sin (q R)-q R \cos (q R)}{(q R)^{3}}\right)
$$

and replacing $\frac{4}{3} \pi R^{3}=V$ it follows:

$$
F(q)=3 V \rho\left(\frac{\sin (q R)-q R \cos (q R)}{(q R)^{3}}\right) .
$$

As $V$ and $\rho$ are both constants the form factor of a solid and homogeneous sphere is described by:

$$
F(q)=3\left(\frac{\sin (q R)-q R \cos (q R)}{(q R)^{3}}\right) .
$$

Fig. 2.9 shows the calculated form factors of a sphere of radius $R=25 \mathrm{~nm}$, an infinitely thin disc of radius $R=25 \mathrm{~nm}$, an infinitely thin rod of length $L=25 \mathrm{~nm}$ and a cylinder of radius $R=25 \mathrm{~nm}$ and length $L=100 \mathrm{~nm}$ using the formulas taken from $[51,57,58]$ : 
- Infinitely thin disk with radius $R$ :

$$
F(q)=\frac{2}{(q R)^{2}}\left(1-\frac{B_{1}(2 q R)}{q R}\right)
$$

where $B_{1}$ is the first order Bessel function.

- Infinitely thin rod with length $L$ :

$$
F(q)=\frac{2 \operatorname{Si}(q L)}{(q L)}-\frac{\left.4 \sin ^{2}(q L / 2)\right)}{(q L)^{2}}
$$

where

$$
\operatorname{Si}(x)=\int_{0}^{x} t^{-1} \sin (t) d t
$$

- Cylinder with radius $R$ and length $L$ :

$$
F(q)=\int_{0}^{\pi / 2}\left[\frac{2 B_{1}(q R \sin \alpha)}{q R \sin \alpha} \frac{\sin (q L \cos \alpha / 2)}{q L \cos \alpha}\right]^{2} \sin \alpha d \alpha
$$

When the dilute solution contains particles identical in size and shape, the total form factor is given by the form factor of one particle multiplied by the number of particles in the solution [51].

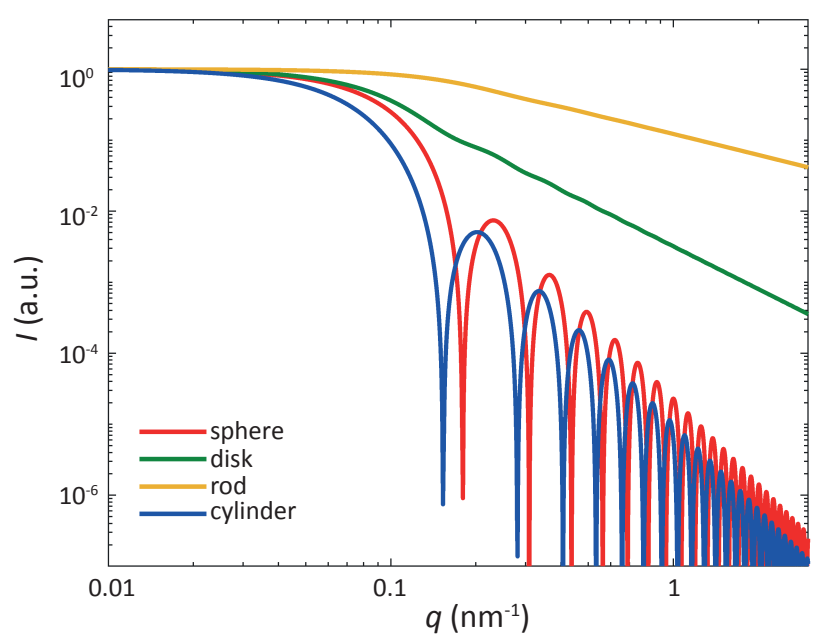

Figure 2.9: Calculated form factors of different shaped objects. A sphere (red, $R=25 \mathrm{~nm}$ ), an infinitely thin disc (green, $R=25 \mathrm{~nm}$ ), an infinitely thin rod (yellow, $L=25 \mathrm{~nm}$ ) and a cylinder (blue $R=25 \mathrm{~nm} ; L=100 \mathrm{~nm}$ ).

However, if the particles do not have the same size or shape, the form factors of all particles are summed and the defined minima of each form factor are smeared out. This phenomenon 
is called polydispersity $[51,55]$. An example is given in Fig. 2.10, where the scattering curve of spheres with three different radii are shown $(R=10 \mathrm{~nm} ; 15 \mathrm{~nm} ; 20 \mathrm{~nm})$, as well as an average curve of all three to mimic polydispersity. It can be observed that the low $q$-value regime is unchanged, however, the minima are smeared out.

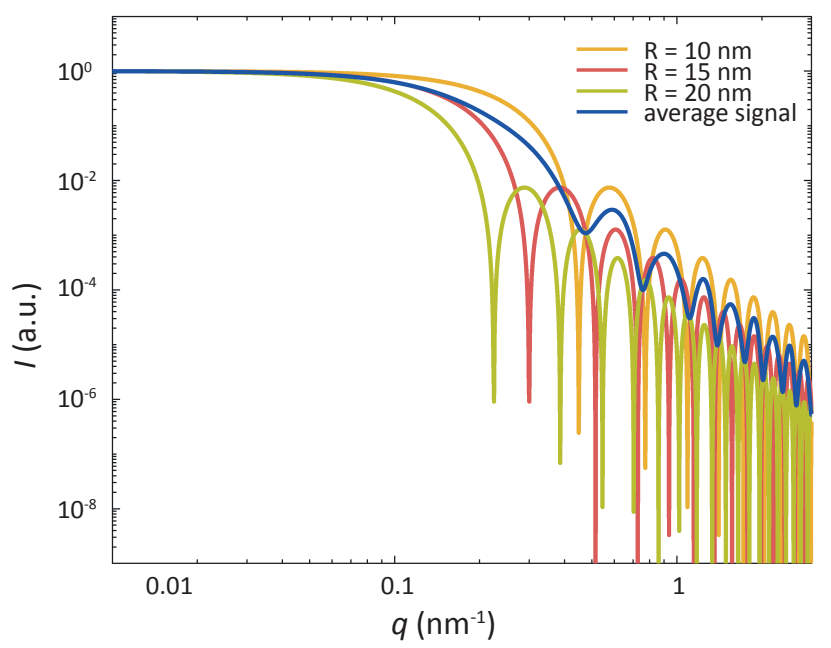

Figure 2.10: Example of polydispersity. To visualize polydispersity, the form factors for three differently sized spheres $(R=10 \mathrm{~nm}, 15 \mathrm{~nm}$ and $20 \mathrm{~nm}$ ) are calculated (yellow, red and green curves respectively) and the average is calculated (blue curve).

\section{The Structure Factor}

$S(q)$, also called "lattice symmetry", is mostly dominant in crystalline structures. If a sample is densely packed and the distances between different particles are on the same length scales as distances within a particle, the resulting scattering pattern is influenced by the neighboring particles as well. The structure factor provides information about the particle position with respect to other particles. In liquid samples, the structure factor describes the strength of interaction between two particles. In an uncorrelated sample, the structure factor $S(q)=1$, thus the scattering signal is only dependent of the form factor [56].

\subsubsection{SAXS Instruments}

All SAXS setups have the same basic components, comprising an X-ray source, a collimation setup and beam focusing optics, as well as a detector to record the data. There are different types of SAXS instruments available. For in-house experiments a Kratky camera setup, the Bonse-Hart instrument or a slit- or pinhole collimation setup can be used [49]. All those instruments produce X-rays in a X-ray tube. When the electrons are emitted by the cathode, the accelerated 
electrons hit the anode and thereby, X-rays are generated. The wavelength of the emitted Xrays is defined by the anode material. To further improve the power and life-time of a source, rotating anodes have been developed [49-52]. The anode rotates and thus the wear per area is reduced. For high quality measurements the generated X-rays should be as monochromatic as possible, as otherwise the scattering effects would be smeared out [51]. In-house, X-ray mirrors or metal foils can be used to monochromatize the beam [49]. Furthermore, the beam needs to be shaped and cleaned from parasitic scattering. Therefore, a good collimation system is of high importance for a good SAXS experiment. To shape the beam and make it less divergent, the beam can either be collimated by pinholes or by slits. The point collimation yields a circular beam with a relatively small beam size, however the measurement time is rather long due to a low primary intensity [52]. Line collimation systems have a long but thin beam, which has a higher scattering intensity than point collimated beams, however the large beam causes slit smearing [52].

Even though in-house machines are constantly improved, it is sometimes necessary to perform the experiment at a synchrotron. Synchrotrons have a much higher photon flux and smaller beam sizes than in-house sources. At synchrotrons particle accelerators are used and the electrons are stored in a storage ring. Radiation is produced in bending magnets [51, 52, 56]. By placing wigglers or undulators between the bending magnets, in the straight part of the storage ring radiation can be produced as well [51]. The radiation produced is not monochromatized, however, by using single-crystal monochromators the wavelength distribution is much more narrow than what can be achieved with mirrors or metal foils. Even if the wavelength distribution of the beam at the synchrotron is more narrow compared to in-house setups the beam needs to be focused. Especially if a high primary intensity is needed at the sample. Slits and curved mirrors are often used to focus the beam. Furthermore, beryllium compound refractive lenses [59] or multilayer zone plates [60] are used as well to focus the beam.

\subsubsection{Measurement Principle and Data Treatment}

In the following a short explanation will be given, on how a SAXS measurement for particles in solution is performed. It has already been mentioned that with X-rays every electron within the illuminated area contributes to the scattering signal and thus to the recorded signal. This is also true of the solvent in which the particles of interest are solubilized as well as the container, e.g., the capillary in which the solution is filled. Therefore, the actual experiment consists of two measurements. First, the container filled with only the solvent and afterwards the container filled with the solvent plus the particles of interest is measured. To obtain the signal of the particles alone, the container measurement needs to be subtracted (Fig. 2.11). 

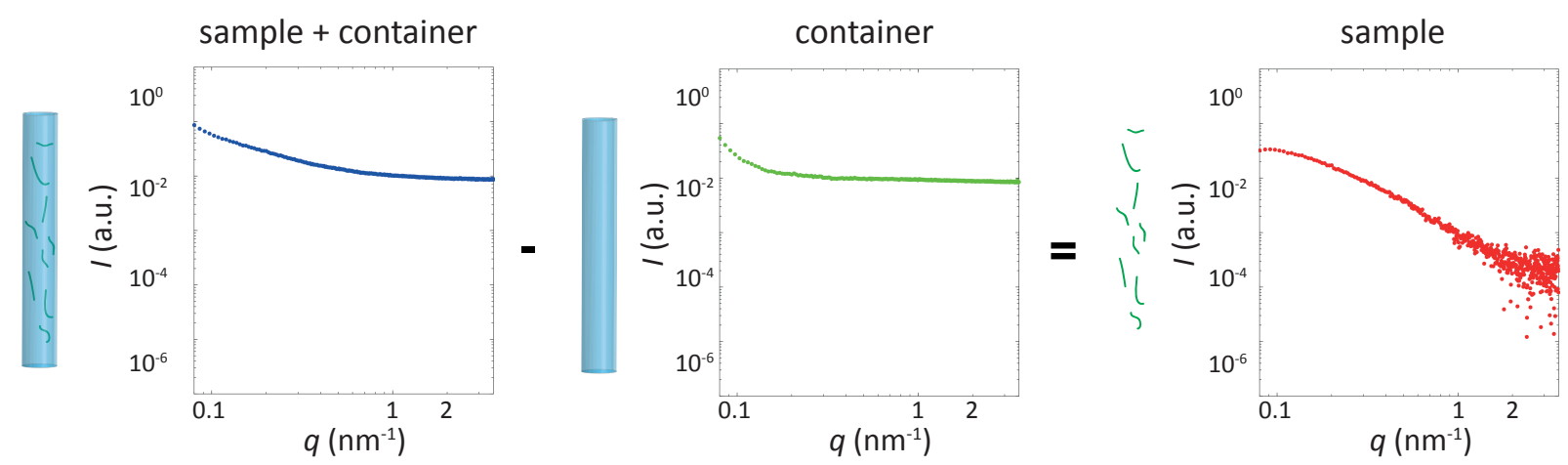

Figure 2.11: Principle of the background subtraction. For solution SAXS measurements the sample of interest is in solution inside a container. To retrieve the signal of the sample alone, the background (solution + container) is measured separately and needs to be subtracted.

Additionally, the SAXS signals should be corrected to improve the data quality. Data should be corrected by the transmission values [49]:

$$
T=\frac{I_{1}}{I_{0}}
$$

where $I_{0}$ is the incident beam and $I_{1}$ the incident beam with the sample placed in the beam path. Furthermore, the signal should be corrected for the exposure time $t_{s}$, the thickness of the sample $d_{s}$ and the concentration of the sample. In a last step, the data can be brought to absolute scale $\left(\mathrm{cm}^{-1}\right)$ by multiplying the data by the correction factor (CF). The correction factor is specific for the used setup $[49,54,61,62]$. By bringing the data on absolute scale, e.g. the molecular weight, the volume fractions or the specific surface area of the scatterers can be determined. A detailed description on how to calculate the CF is given in Appendix A.

\subsubsection{Data Analysis}

For analyzing SAXS data, different regimes can be evaluated yielding different types of information. Which region can be analyzed and what information can be gained, depends on the data quality and on the measured $q$-range. In general, three different regions can be analyzed (the Guinier region, the intermediate region and the Porod region). By analyzing the Guinier region, information about the size of the scatterers can be retrieved. Analyzing the intermediate region, shape information are retrieved and by analyzing the Porod region surface information is gained. Furthermore, the whole scattering curve can be analyzed by using a model. In the following, several analyzing methods will be explained briefly. 


\section{Guinier's Approximation and Pair Distance Distribution Function}

To characterize the size of the particle (sample), Guinier approximation or the pair distance distribution function (pddf) can be utilized. If the measurement is good enough in quality the $I(0)$ value as well as the radius of gyration $\left(R_{g}\right)$ can be extracted. $I(0)$ is the intensity scattered to zero angle, which cannot be measured but obtained by extrapolation of the scattering profile [48]. It is related to the molecular weight by:

$$
I(0)=\frac{c \Delta \rho^{2} v^{2} \mathrm{MW}}{N_{A}} .
$$

With $c$ as the particle concentration, $v$ the particles specific volume, MW the molecular weight and $N_{A}$ Avogadro's number. $\Delta \rho$ is the scattering contrast, which is the electron density of the particle and the solution $\left(\Delta \rho=\rho_{\text {particle }}-\rho_{\text {solution }}\right)[48,54] . R_{g}$ is defined as the root-mean-squared distance from the center of gravity of a particle [51].

For the Guinier analysis only the very small $q$-values are taken into account. Guinier showed that data can be fitted linearly when plotting $\ln (I(q))$ against $q^{2}$. The $R_{g}$ values can be retrieved from the slope of the fit, whereas the intercept with the $I(q)$ axis denotes to the $I(0)$ value [48, 51]. The following relations were found for small $q$-values and are important for this thesis [51, 58]:

- Sphere:

$$
I(q)=I(0) \exp \left(\frac{-q^{2} R_{g}^{2}}{3}\right)
$$

with the corresponding radius of gyration:

$$
R_{g}^{2}=\frac{3}{5} R^{2}
$$

- Cylinder:

$$
I(q)=\frac{I(0)}{q} \exp \left(\frac{-q^{2} R_{c}^{2}}{2}\right)
$$

and the corresponding radius of gyration:

$$
R_{g}^{2}=\frac{R^{2}}{2}+\frac{L^{2}}{12}
$$

The Guinier region is defined by limiting the value of the product $q R_{g}$. The limit for the upper bound of $q R_{g}$ is strongly dependent on the shape of the sample. The $q R_{g}$ limit for spherical shapes is 1.3, whereas for elongated objects the limit is smaller. A limit of $q R_{g} \leq 1$ is described 
in literature [56]. However, in biological SAXS this limit is extended to $q R_{g} \leq 1.3$, due to the low numbers of included points for the limit of $q R_{g} \leq 1[55,63]$. Another requirement which is often neglected when performing Guinier analysis is that there should not be any interaction between particles in the sample [56]. If the SAXS curve at low $q$-values increases (upturn), it is an indication for attraction within the sample, whereas a downturn of the intensity at low $q$ values is an indication for repulsive interaction [64-66].

Another way to calculate the radius of gyration as well as the radius in real space, the pddf $(p(r))$ can be applied. By calculating $p(r)$ the whole scattering curve is taken into account. By an indirect Fourier transformation, data are represented in real space [50, 55]. From the pddf information about all distances between atoms within the particle can be retrieved [65]. To retrieve dimension information of a particle (e.g. size) the angles must be smaller than $q=\pi / D_{\max }$, where $D_{\max }$ refers to the largest particle diameter in the sample [65]. For analysis of the pddf, one needs to know $D_{\max }$, which can be difficult in an unknown system like assembling protein. However, if $D_{\max }$ is known and with the assumptions that $p(0)=0$ and the function is not negative at $D_{\max }$, the radius of gyration can be calculated with [65]:

$$
R_{g}^{2}=\frac{\int_{0}^{D_{\max }} r^{2} p(r) d r}{2 \int_{0}^{D_{\max }} p(r) d r}
$$

\section{Porod Analysis}

Analysis of the Porod region is only valid if $q R_{g} \gg 1$, thus for large $q$-values. In this regime the intensity is proportional to the surface area. Furthermore, the intensity at large $q$-values decays with $K_{P} / q^{4}$, where $K_{P}$ is the Porod constant. To analyze the Porod regime, the Porod Invariant:

$$
Q=\int_{0}^{\infty} q^{2} I(q) d q=2 \pi^{2} \int_{V}(\Delta \rho(\vec{r}))^{2} d \vec{r},
$$

can be used, which is equal in reciprocal and in real space [55]. With this assumption both the Porod volume and the specific surface can be calculated on a relative scale [55]. The scattering profile in the Porod regime follows a linear trend when $q^{4} I(q)$ is plotted against $q^{4}$ [55].

\subsubsection{Applications of SAXS}

Covering length scales in the micrometer and nanometer range and retrieving size, shape and surface information of the sample investigated, SAXS is a useful tool used in various fields. As the sample can be solid or liquid and no labeling is required biological samples can be measured directly in their natural environment. SAXS is often used for biological samples to e.g. investigate: protein folding [65, 67], vesicle fusion or adhesion [68] or the assembly processes 
of proteins. In static SAXS experiments, Brennich et al. and Hémonnot et al. investigated the influence of $\mathrm{KCl}$ and $\mathrm{MgCl}_{2}$ on the assembly of the IF proteins vimentin and keratin respectively $[24,25]$. They showed that with increasing ion concentration the filament radius increases and furthermore, the effect of $\mathrm{MgCl}_{2}$ was stronger than with $\mathrm{KCl}$. This systematic investigation of different ion concentrations leading to $\mathrm{nm}$ changes in the filament radius is well detectable with SAXS.

\subsection{Microfluidics}

With microfluidics, the behavior of single or multiple, interacting fluids as well as reagents can be studied. Microfluidics can be used to establish stable 2D or even 3D gradients to study directed cell migration [69], chemotaxis of bacteria for cancer targeting [70] or the self-assembly of collagen in a pH gradient [71]. Another example for the use of microfluidic devices is to follow protein folding [72]. In this section, an introduction to the fundamental physics of microfluidics will be given.

\subsubsection{Flow at Low Reynolds Number}

Most studies using microfluidic devices use incompressible Newtonian fluids such as water. The motion of the flow of an incompressible Newtonian fluid follows the Navier-Stokes equation, which describes the fluid motion:

$$
\rho(\vec{r}, t)\left(\frac{\partial \vec{v}}{\partial t}+(\vec{v} \vec{\nabla}) \vec{v}\right)=-\vec{\nabla} p+\eta \nabla^{2} \vec{v}+\vec{F}_{e x},
$$

where $\rho$ is the fluid density, $\vec{v}$ the velocity of the fluid, $t$ the time, $p$ the pressure, $\eta$ the viscosity and $\vec{F}_{e x}$ external forces. Due to the non-linear term $\rho(\vec{v} \cdot \nabla) \vec{v}$, it is not possible to solve the equation analytically. The Navier-Stokes equation relates the inertial forces of the fluid (left hand side of the equation) with the viscous forces (right hand side) $[73,74]$. The ratio between the inertial forces and the viscous forces is described by a dimensionless number known as the Reynolds number $(R e)[73,74]$ :

$$
R e=\frac{\rho v L}{\eta},
$$

where $\mathrm{L}$ is the characteristic length scale. Depending on whether the inertial or the viscous forces dominate the flow, different flow profiles can be observed. At $R e \ll 1$ viscous forces dominate, meaning the inertial forces can be neglected. The flow is then called laminar flow and is typical in microfluidics as the length scales are in the range of $\mu \mathrm{m}$. Above $R e \gg 1$ the inertial 
forces dominate. Turbulent flow occurs if $R e>2300$ [75], however the value can be smaller if the flow is disturbed for example by obstacles [73]. In the laminar flow regime the inertial forces can be neglected and the flow is time independent and linear. Furthermore, if there are no external forces the Navier-Stokes equation is reduced to the Stokes equation:

$$
\nabla p=\eta \nabla^{2} \vec{v}
$$

\subsubsection{Mixing in Microfluidic Devices}

When mixing wants to be investigated in a microfluidic device, the mass transport (e.g. transport of chemicals, proteins) needs to be considered. Mass transport is described by two processes; convection and diffusion. Convection describes the particle motion in relation to the flow. Diffusion $(D)$ describes the particle motion without any active mass transport and can be described by Fick's first law:

$$
\vec{j}_{\text {diff }}=-D \nabla c
$$

where $D$ is the diffusion and $c$ the concentration. Fick's first law describes that the fluid flux is proportional to the concentration gradient. The flux of the convection can be described by:

$$
\vec{j}_{\text {conv }}=\vec{v} c,
$$

which is simply the product of the flow field and the concentration. The total flux of a system can be described as the sum of Fick's first law and the convectional flux. Inserting the total flux in the continuity equation

$$
\frac{\partial \rho}{\partial t}=-\nabla(\rho \vec{v})
$$

where the concentration corresponds to the density, the convection diffusion equation can be derived:

$$
\frac{\partial c}{\partial t}=\nabla(D \nabla c)-\nabla(\vec{v} c)
$$

For a particle, the diffusion can be described by the Einstein and Smoluchowsky relation:

$$
D=\mu k_{B} T
$$

where $\mu$ is the mobility of the particle, $k_{B}$ is the Boltzmann constant and $T$ the temperature. For small spheres Stokes found: 


$$
\mu=\frac{1}{6 \pi \eta R_{h}},
$$

where $R_{h}$ is the hydrodynamic radius of the particle. Combining the relation Stokes found with the Einstein and Smoluchowsky equation, the diffusion constant for small spheres can be described as follows [74]:

$$
D=\frac{k_{B} T}{6 \pi \eta R_{h}}
$$

with $D$ having the unit $\mathrm{m}^{2} / \mathrm{s}$. With Equation 2.40 it can be observed that the diffusion of a small particle is faster than for a large particle.

Just like the Reynolds number describes the relationship between inertial and viscous forces, the Péclet number describes the relation between the diffusive time and the convective time, meaning the transport of solutes:

$$
P e=\frac{v L}{D} .
$$

Thus the Péclet number is dependent on the fluid velocities, the characteristic length scale and the diffusion coefficient. If the Péclet number is large, the flow follows the flow lines and no mixing is induced. If the Péclet number is small, diffusion is dominant and the mixing is achieved through diffusion. By changing the characteristic length or the flow speed, mixing can be induced.

\subsubsection{Microfluidic Devices for Diffusive Mixing}

Replicating biological processes in microfluidic channels is nowadays common as normally large-scale processes can be scaled down to small devices. A benefit is that only small sample volumes are needed. By manipulating processes in a controlled way, microfluidics are used to investigate unknown processes. The microfluidic chip can hold a single channel or a set of multiple channels connected with each other. By flowing liquids through the channels, the fluids can be mixed or separated. On a macromolecular scale turbulence is used to mix fluids. This is however not applicable in the laminar flow regime, due to the small Reynolds number. Thus, mixing is diffusion driven. As the diffusion time cannot be reduced, a faster mixing can be achieved by reducing the distance the particles have to diffuse.

In the work here, the assembly process of IFs is studied. By flushing protein through a microfluidic channel and mixing it with assembly reagents $(\mathrm{KCl})$, the assembly of the IF is initiated [76]. As a fast mixing of the protein with the assembly reagent is desirable the diffusion length should 
be as short as possible. The diffusion of the protein is on the order of $10^{-11} \mathrm{~m}^{2} / \mathrm{s}$ and the diffusion of $\mathrm{KCl}$ on the order of $10^{-9} \mathrm{~m}^{2} / \mathrm{s}$. Thus, the mixing is mainly dependent on the diffusion of $\mathrm{KCl}$. To achieve a fast mixing, the protein stream should be as narrow as possible. Once the assembly is initiated, the assembly process over time can be investigated by observing the protein in flow. However, the early assembly steps of IFs appear on length scales in the nm-range and therefore, can not be observed with light microscopy. This is one reason why the combination of microfluidics and SAXS is gaining more interest. A challenge is that many device materials are not compatible with SAXS and therefore, there is still a need for reproducible, X-ray compatible, inexpensive and easy-to-make microfluidic devices [77]. The chip itself can be fabricated from many different materials e.g glass, polydimethylsiloxane (PDMS) or plastic [78]. Especially for microscopy experiments PDMS and glass are often used as the devices are easy to fabricate, optically transparent and inexpensive. However, PDMS is not suitable for X-ray experiments. A more detailed description on the needs of microfluidic devices when combined with X-rays and what can be investigated is given in Chapter 6 . 


\section{References}

1. Herrmann, H. \& Aebi, U. Intermediate filaments and their associates: multi-talented structural elements specifying cytoarchitecture and cytodynamics. Curr. Opin. Cell Biol. 12, 7990 (2000).

2. Herrmann, H., Strelkov, S. V., Burkhard, P. \& Aebi, U. Intermediate filaments: primary determinants of cell architecture and plasticity. J. Clin. Invest. 119, 1772-1783 (2009).

3. Herrmann, H. \& Aebi, U. Intermediate Filaments: Structure and Assembly. Cold Spring Harbor Perspect. Biol. 8, a018242 (2016).

4. Conway, J. F. \& Parry, D. A. Intermediate filament structure: 3. Analysis of sequence homologies. Int. J. Biol. Macromol. 10, 79-98 (1988).

5. Forgacs, G., Yook, S. H., Janmey, P. A., Jeong, H. \& Burd, C. G. Role of the cytoskeleton in signaling networks. J. Cell Sci. 117, 2769-2775 (2004).

6. Janmey, P. A. The Cytoskeleton and Cell Signaling: Component Localization and Mechanical Coupling. Physiol. Rev. 78, 763-781 (1998).

7. Alberts, B. et al. Molecular Biology of the Cell Sixth edition (Garland Science Taylor and Francis Group, New York, NY, 2015).

8. Huber, F., Boire, A., López, M. P. \& Koenderink, G. H. Cytoskeletal crosstalk: when three different personalities team up. Curr. Opin. Cell Biol. 32, 39-47 (2015).

9. Herrmann, H. \& Aebi, U. Intermediate Filaments: Molecular Structure, Assembly Mechanism, and Integration Into Functionally Distinct Intracellular Scaffolds. Annu. Rev. Biochem. 73, 749-789 (2004).

10. Wen, Q. \& Janmey, P. A. Polymer physics of the cytoskeleton. Curr. Opin. Solid State Mater. Sci. 15, 177-182 (2011).

11. Block, J., Schroeder, V., Pawelzyk, P., Willenbacher, N. \& Köster, S. Physical properties of cytoplasmic intermediate filaments. Biochim. Biophys. Acta, Mol. Cell Res. 1853, 30533064 (2015).

12. Fuchs, E. \& Weber, K. Intermediate Filaments: Structure, Dynamics, Function and Disease. Annu. Rev. Biochem. 63, 345-382 (1994).

13. Szeverenyi, I. et al. The Human Intermediate Filament Database: comprehensive information on a gene family involved in many human diseases. Hum. Mutat. 29, 351-360 (2008).

14. Chernyatina, A. A., Guzenko, D. \& Strelkov, S. V. Intermediate filament structure: the bottom-up approach. Curr. Opin. Cell Biol. 32, 65-72 (2015). 
15. Strelkov, S. V. \& Burkhard, P. Analysis of Alpha-Helical Coiled Coils with the Program TWISTER Reveals a Structural Mechanism for Stutter Compensation. J. Struct. Biol. 137, 54-64 (2002).

16. Köster, S., Weitz, D. A., Goldman, R. D., Aebi, U. \& Herrmann, H. Intermediate filament mechanics in vitro and in the cell: from coiled coils to filaments, fibers and networks. Curr. Opin. Cell Biol. 32, 82-91 (2015).

17. Herrmann, H. et al. Structure and Assembly Properties of the Intermediate Filament Protein Vimentin: The Role of its Head, Rod and Tail Domains. J. Mol. Biol. 264, 933-953 (1996).

18. Kreplak, L. \& Fudge, D. Biomechanical properties of intermediate filaments: from tissues to single filaments and back. Bioessays 29, 26-35 (2006).

19. Steinert, P. M., Idler, W. W. \& Zimmerman, S. B. Self-assembly of bovine epidermal keratin filaments in vitro. J. Mol. Biol. 108, 547-567 (1976).

20. Kornreich, M., Avinery, R., Malka-Gibor, E., Laser-Azogui, A. \& Beck, R. Order and disorder in intermediate filament proteins. FEBS Lett. 589, 2464-2476 (2015).

21. Lichtenstern, T., Mücke, N., Aebi, U., Mauermann, M. \& Herrmann, H. Complex formation and kinetics of filament assembly exhibited by the simple epithelial keratins K8 and K18. J. Struct. Biol. 177, 54-62 (2012).

22. Winheim, S. et al. Deconstructing the Late Phase of Vimentin Assembly by Total Internal Reflection Fluorescence Microscopy (TIRFM). PLOS ONE 6 (ed Kreplak, L.) e19202 (2011).

23. Dammann, C., Herrmann, H. \& Köster, S. Competitive Counterion Binding Regulates the Aggregation Onset of Vimentin Intermediate Filaments. Isr. J. Chem. 56, 614-621 (2015).

24. Brennich, M. E. et al. Impact of ion valency on the assembly of vimentin studied by quantitative small angle X-ray scattering. Soft Matter 10, 2059-2068 (2014).

25. Hémonnot, C. Y. J., Mauermann, M., Herrmann, H. \& Köster, S. Assembly of Simple Epithelial Keratin Filaments: Deciphering the Ion Dependence in Filament Organization. Biomacromolecules 16, 3313-3321 (2015).

26. Herrmann, H., Häner, M., Brettel, M., Ku, N.-O. \& Aebi, U. Characterization of distinct early assembly units of different intermediate filament proteins. J. Mol. Biol. 286, 1403-1420 (1999).

27. Strelkov, S. V., Herrmann, H. \& Aebi, U. Molecular architecture of intermediate filaments. BioEssays 25, 243-251 (2003). 
28. Mücke, N. et al. Molecular and Biophysical Characterization of Assembly-Starter Units of Human Vimentin. J. Mol. Biol. 340, 97-114 (2004).

29. Nöding, B. \& Köster, S. Intermediate Filaments in Small Configuration Spaces. Phys. Rev. Lett. 108 (2012).

30. Premchandar, A. et al. Analysis of distinct molecular assembly complexes of keratin K8 and K18 by hydrogen-deuterium exchange. J. Struct. Biol. 192, 426-440 (2015).

31. Dammann, C., Nöding, B. \& Köster, S. Vimentin networks at tunable ion-concentration in microfluidic drops. Biomicrofluidics 6, 022009 (2012).

32. Lopez, C. G., Saldanha, O., Huber, K. \& Köster, S. Lateral association and elongation of vimentin intermediate filament proteins: A time-resolved light-scattering study. P. Natl. Acad. Sci. U.S.A. 113, 11152-11157 (2016).

33. Hofmann, I., Herrmann, H. \& Franke, W. W. Assembly and structure of calcium-induced thick vimentin filaments. Eur. J. Cell Biol. 56, 328-341 (1991).

34. Strelkov, S. V., Schumacher, J., Burkhard, P., Aebi, U. \& Herrmann, H. Crystal Structure of the Human Lamin A Coil 2B Dimer: Implications for the Head-to-tail Association of Nuclear Lamins. J. Mol. Biol. 343, 1067-1080 (2004).

35. Herrmann, H., Kreplak, L. \& Aebi, U. in Intermediate Filament Cytoskeleton (eds Omary, M. B. \& Coulombe, P. A.) 3-24 (Academic Press, 2004).

36. Singer, C. Notes on the Early History of Microscopy. Proc. R. Soc. Med. 7, 247-279 (1914).

37. Principles of Fluorescence Spectroscopy 3rd ed. (ed Lakowicz, J. R.) (Springer US, 1983).

38. Noomnarm, U. \& Clegg, R. M. Fluorescence lifetimes: fundamentals and interpretations. Photosynth. Res. 101, 181-194 (2009).

39. Jabłoński, A. Über den Mechanismus der Photolumineszenz von Farbstoffphosphoren. Zeitschrift für Physik 94, 38-46 (1935).

40. Toseland, C. P. Fluorescent labeling and modification of proteins. J. Chem. Biol. 6, 85-95 (2013).

41. Hölscher, H., Allers, W., Schwarz, U. D., Schwarz, A. \& Wiesendanger, R. Interpretation of "true atomic resolution" images of graphite (0001) in noncontact atomic force microscopy. Phys. Rev. B 62, 6967-6970 (2000).

42. Rugar, D. \& Hansma, P. Atomic Force Microscopy. Phys. Today 43, 23-30 (1990).

43. Oliveira, R. D., Albuquerque, D., Cruz, T., Yamaji, F. \& Leite, F. in Atomic Force Microscopy Imaging, Measuring and Manipulating Surfaces at the Atomic Scale (InTech, 2012). 
44. Alsteens, D. et al. Atomic force microscopy-based characterization and design of biointerfaces. Nat. Rev. Mater. 2 (2017).

45. Liu, S. \& Wang, Y. in Advances in Food and Nutrition Research 201-240 (Elsevier, 2011).

46. Mücke, N., Kirmse, R., Wedig, T., Leterrier, J. \& Kreplak, L. Investigation of the morphology of intermediate filaments adsorbed to different solid supports. J. Struct. Biol. 150, 268-276 (2005).

47. MacDonald, C. A. An Introduction to X-Ray Physics, Optics, and Applications (Princeton University Press, 2017).

48. Jacques, D. A. \& Trewhella, J. Small-angle scattering for structural biology-Expanding the frontier while avoiding the pitfalls. Protein Sci. 19, 642-657 (2010).

49. Pauw, B. R. The Impossible Project "-How to do a perfect SAXS measurement in (2011).

50. Small angle X-ray Scattering (eds Glatter, O. \& Kratky, O.) (Academic Press, 1982).

51. Als-Nielsen, J. \& McMorrow, D. Elements of Modern X-ray Physics (John Wiley \& Sons, Inc., 2011).

52. H. Schnablegger, Y. S. The SAXS Guide. Getting acquainted with the principles 3rd ed. (Anton Paar GmbH, 2006).

53. Small-Angle Scattering of X-Rays (eds Guinier, A. \& Fournet, G.) (John Wiley and Sonst. Inc., 1955).

54. Orthaber, D., Bergmann, A. \& Glatter, O. SAXS experiments on absolute scale with Kratky systems using water as a secondary standard. J. Appl. Crystallogr. 33, 218-225 (2000).

55. Svergun, D. I. \& Koch, M. H. J. Small-angle scattering studies of biological macromolecules in solution. Rep. Prog. Phys. 66, 1735-1782 (2003).

56. Neutron and Synchrotron Radiation for Condensed Matter Studies. Theory, Instruments and Methods (eds Baruchel, J., Hodenau, J., Lehmann, M., Regnard, J. \& Schenker, C.) (EDP Sciences - Springer-Verlag, 2010).

57. Pedersen, J. S. Analysis of small-angle scattering data from colloids and polymer solutions: modeling and least-squares fitting. Adv. Colloid Interface Sci. 70, 171-210 (1997).

58. Porod, G. Die Röntgenkleinwinkelstreuung von dichtgepackten kolloiden Systemen. Kolloid-Zeitschrift 125, 51-57 (1952).

59. Schroer, C. G. et al. Hard x-ray nanoprobe based on refractive x-ray lenses. Appl. Phys. Lett. 87, 124103 (2005). 
60. Osterhoff, M., Eberl, C., Soltau, J. \& Krebs, H.-U. Faster scanning and higher resolution: new setup for multilayer zone plate imaging in X-Ray Nanoimaging: Instruments and Methods III (eds Lai, B. \& Somogyi, A.) (SPIE, 2017).

61. Dreiss, C. A., Jack, K. S. \& Parker, A. P. On the absolute calibration of bench-top small-angle X-ray scattering instruments: a comparison of different standard methods. J. Appl. Crystallogr. 39, 32-38 (2006).

62. Fan, L., Degen, M., Bendle, S., Grupido, N. \& Ilavsky, J. The Absolute Calibration of a SmallAngle Scattering Instrument with a Laboratory X-ray Source. J. Phys.: Conf. Ser. 247, 012005 (2010).

63. Mertens, H. D. \& Svergun, D. I. Structural characterization of proteins and complexes using small-angle X-ray solution scattering. J. Struct. Biol. 172, 128-141 (2010).

64. Skou, S., Gillilan, R. E. \& Ando, N. Synchrotron-based small-angle X-ray scattering of proteins in solution. Nat. Protoc. 9, 1727-1739 (2014).

65. Kikhney, A. G. \& Svergun, D. I. A practical guide to small angle X-ray scattering (SAXS) of flexible and intrinsically disordered proteins. FEBS Lett. 589, 2570-2577 (2015).

66. Tuukkanen, A. T., Spilotros, A. \& Svergun, D. I. Progress in small-angle scattering from biological solutions at high-brilliance synchrotrons. IUCrJ 4, 518-528 (2017).

67. Schroer, M. A. et al. High-Pressure SAXS Study of Folded and Unfolded Ensembles of Proteins. Biophys. J. 99, 3430-3437 (2010).

68. Komorowski, K. et al. Vesicle Adhesion and Fusion Studied by Small-Angle X-Ray Scattering. Biophys. J. 114, 1908-1920 (2018).

69. Saadi, W. et al. Generation of stable concentration gradients in 2D and 3D environments using a microfluidic ladder chamber. Biomed. Microdevices 9, 627-635 (2007).

70. Song, J. et al. A microfluidic device for studying chemotaxis mechanism of bacterial cancer targeting. Sci. Rep. 8 (2018).

71. Köster, S., Evans, H. M., Wong, J. Y. \& Pfohl, T. An in-situ Study of Collagen Self-Assembly Processes. Biomacromolecules 9, 199-207 (2008).

72. Waldauer, S. A., Wu, L., Yao, S., Bakajin, O. \& Lapidus, L. J. Microfluidic Mixers for Studying Protein Folding. J. Visualized Exp. (2012).

73. Wieghardt, K. Theoretische Strömungslehre (Göttingen University Press, 2005).

74. Bruus, H. Theoretical microfludics (Oxford University Press, 2008). 
75. Beebe, D. J., Mensing, G. A. \& Walker, G. M. Physics and Applications of Microfluidics in Biology. Annu. Rev. Biomed. Eng. 4, 261-286 (2002).

76. Brennich, M. E. et al. Dynamics of intermediate filament assembly followed in micro-flow by small angle X-ray scattering. Lab Chip 11, 708-716 (2011).

77. Ghazal, A. et al. Recent advances in X-ray compatible microfluidics for applications in soft materials and life sciences. Lab Chip 16, 4263-4295 (22 2016).

78. Duffy, D. C., McDonald, J. C., Schueller, O. J. A. \& Whitesides, G. M. Rapid Prototyping of Microfluidic Systems in Poly(dimethylsiloxane). Anal. Chem. 70. PMID: 21644679, 49744984 (1998). 


\section{Chapter 3}

\section{Materials and Methods}

All chemicals used and their full names are listed in Table 3.5 at the end of this Chapter. For the preparation of buffers and solutions ultrapure-water is used unless otherwise stated. SAXS and AFM experiments are performed with the human vimentin wild-type (wt), meaning not genetically modified protein. Plasmid sequencing shows a mutation at position 2 where the amino acid serine is replaced by alanine. For fluorescence imaging experiments, vimentin wt is mixed with a genetically modified version of human vimentin. In vimentin C328A, the cysteine at position 328 is genetically replaced by alanine. Additionally, 2 glycines and a cysteine are incorporated at the C-terminal of the protein. Vimentin C328A is labeled with ATTO647N-malemide dye by Anna Schepers following the protocols described in [1, 2]. Plasmids for both proteins are provided by Harald Herrmann.

\subsection{Protein Reconstitution}

Protein purification is conducted by Susanne Bauch following the protocol listed in [3]. Human vimentin wt as well as human vimentin C328A is expressed in Escherichia coli bacteria and purified from inclusion bodies. The purified protein is stored in $8 \mathrm{M}$ urea, $5 \mathrm{mM}$ Tris(hydroxymethyl)aminomethan $\mathrm{HCl}$ (TRIS- $\mathrm{HCl}$ ) at a $\mathrm{pH}$ of 7.5, $1 \mathrm{mM}$ ethylenediaminetetraacetic acid (ETDA), $0.1 \mathrm{mM}$ ethylene glycol-bis(2-aminoethylether)-N, N,N',N'-tetraacetic acid (EGTA), $1 \mathrm{mM}$ 1,4-di-thiothreitol (DTT) and $10 \mathrm{mM}$ methylamine hydrochloride (MAC) at $-80^{\circ} \mathrm{C}$. The storage buffer consists of agents conserving the protein and urea, which keeps the protein denatured. Two sets of experiments are performed. First the influence of buffers or different ions on the protein is tested in a steady-state experiment. In a second experiment the assembly process in flow is observed. To this aim, the protein is reconstituted and urea is dialyzed from the protein solution. Dialysis is performed to transfer the protein from the stor- 
age buffer containing $8 \mathrm{M}$ urea to the desired buffer without urea. Table 3.1 shows the three buffers used: Phosphate buffer (PB), 3-(N-morpholino)propanesulfonic acid (MOPS), and TRIS with their concentrations and their respective $\mathrm{pH}$ values. The dialysis is performed in a step-

Table 3.1: Overview of all used buffers and their respective concentrations and $p H$ values.

\begin{tabular}{lll}
\hline Buffer & Concentration $(\mathrm{mM})$ & $\mathrm{pH}$ \\
\hline Phosphate Buffer & 2 & 8.4 \\
& 2 & 7.5 \\
& 20 & 7.5 \\
TRIS-HCl & 2 & 7.5 \\
& 20 & 7.5 \\
MOPS-NaCl & 2 & 7.5 \\
& 20 & 7.5 \\
\hline
\end{tabular}

wise manner at $21^{\circ} \mathrm{C}$. The protein is filled in $50 \mathrm{kDa}$ cut-off dialysis tubing (SpectraPor, CarlRoth $\mathrm{GmbH}$, Karlsruhe, Germany), which is placed in a beaker with at least $500 \mathrm{~mL}$ desired end buffer (Table 3.1) containing $6 \mathrm{M}$ urea. Every $30 \mathrm{~min}$ the buffer is exchanged so that the protein is dialyzed stepwise into buffer containing 4, 2, 1 and $0 \mathrm{M}$ urea. An additional cleaning step is performed over night at $8^{\circ} \mathrm{C}$. On the following day the protein is stirred for at least $2 \mathrm{~h}$ in fresh buffer at $21^{\circ} \mathrm{C}$. The dialyzed protein is stored at $4{ }^{\circ} \mathrm{C}$ and can be used for experiments up to one week, as it has been reported by Brennich et al. that the scattering patterns do not change within 5 days [4]. The protein concentration is obtained by measuring the absorption at $280 \mathrm{~nm}$ using the NanoDrop. At least three concentration measurements per protein are performed and the arithmetic mean is calculated.

For the dialysis with labeled protein, unlabeled vimentin wt is mixed with labeled vimentin C328A, so that in total $2-5 \%$ of the protein is labeled protein. Reconstitution is performed the same way as for vimentin wt, with the difference that the beaker is shielded from light, to not bleach the fluorophores during the whole process.

\subsection{Protein Assembly}

Protein is assembled using the kick-start method. Both protein and buffer are mixed directly with a pipette. In Chapter 4 the influence of buffer on the assembly is tested. Vimentin is dialyzed in PB, TRIS or MOPS at low $(2 \mathrm{mM})$ and high $(20 \mathrm{mM})$ concentrations and investigated. The tetramers as well as assembled filaments are measured. For assembly $100 \mathrm{mM} \mathrm{KCl}$ (final concentration) are added. In Chapter 5 the influence of ions on the assembly is investigated. For this, vimentin assembled with different ions at various concentrations are measured in $2 \mathrm{mM}$ MOPS buffer at pH 7.5. A list of all ions and their concentrations is found in Table 3.2. 
Table 3.2: Overview of ions used for assembly experiments in $2 \mathrm{mM}$ MOPS buffer at $p H 7.5$ and their respective concentrations during SAXS measurements

\begin{tabular}{lllllllll}
\hline Ion & \multicolumn{7}{c}{ Concentration $(\mathrm{mM})$} \\
$\mathrm{NaCl}$ & 10 & 20 & 30 & 40 & 50 & 80 & 100 & 150 \\
$\mathrm{KCl}$ & 10 & 20 & 30 & 40 & 50 & 80 & 100 & 150 \\
$\mathrm{MgCl}_{2}$ & 0.5 & 1.0 & 1.5 & 2.0 & 2.5 & 4.0 & 5.0 & 10.0 \\
$\mathrm{CaCl}_{2}$ & 0.5 & 1.0 & 1.5 & 2.0 & 2.5 & 4.0 & 5.0 & 10.0 \\
Hexammine-cobalt(III) chloride & 0.01 & 0.02 & 0.03 & 0.05 & 0.08 & 0.1 & 0.2 & 0.5 \\
Spermine & 0.01 & 0.02 & 0.03 & 0.04 & 0.05 & 0.08 & 0.09 & 0.1 \\
\hline
\end{tabular}

\subsubsection{SAXS Experiments}

For static in-house SAXS experiments the protein is diluted to a concentration of $2 \mathrm{mg} / \mathrm{mL}$ unless otherwise stated. Buffers with the 2-fold ion concentrations (doubled amount of ion concentration which are listed in Table 3.2) are prepared. For assembling vimentin with e.g. $100 \mathrm{mM}$ $\mathrm{KCl}, 2 \mathrm{mg} / \mathrm{mL}$ vimentin is mixed with $200 \mathrm{mM} \mathrm{KCl}$ in a ratio of $1: 1$, to have a final protein concentration of $1 \mathrm{mg} / \mathrm{mL}$ and an ion concentration of $100 \mathrm{mM} \mathrm{KCl}$. Measurements are performed using quartz glass capillaries with an outer diameter of $1.5 \mathrm{~mm}$ (Hilgenberg, Malsfeld, Germany). As it is not possible to homogeneously fill the capillary with assembled vimentin, cooled buffers and protein are mixed at a ratio of 1:1 in a reaction tube and transferred directly into the capillary with a microloader pipette tip (Eppendorf AG, Wesseling-Berzdorf, Germany). The final protein concentration during the experiment is $1 \mathrm{mg} / \mathrm{mL}$. The capillary is sealed with wax (Hampton Research, Aliso Viejo, USA) when performing measurements in vacuum to prevent evaporation of the liquid. Protein is assembled in the capillary for at least $8 \mathrm{~h}$ at $21^{\circ} \mathrm{C}$ before the measurements starts.

For time-resolved measurements at the synchrotron, vimentin is reconstituted at a concentration of $5.5 \mathrm{mg} / \mathrm{mL}$. As assembly is initiated in the microfluidic device, no assembly is performed prior to experiments.

\subsubsection{Microscopy Experiments}

For visualization of assembled filaments, fluorescence microscopy and AFM are used. Both methods need a much lower protein concentration than SAXS experiments. For AFM, the protein is diluted to a concentration of $0.2 \mathrm{mg} / \mathrm{mL}$, and for fluorescence microscopy, a concentration of $0.4 \mathrm{mg} / \mathrm{mL}$ is adjusted. The protein is mixed 1:1 in both cases with the 2-fold ion concentration in the assembly buffer (e.g. mix protein with $200 \mathrm{mM} \mathrm{KCl}$ to have a final concentration of $100 \mathrm{mM} \mathrm{KCl}$ ), so that the final protein concentration for AFM is $0.1 \mathrm{mg} / \mathrm{mL}$ and $0.2 \mathrm{mg} / \mathrm{mL}$ for fluorescence microscopy experiments. During AFM experiments, $100 \mathrm{mM} \mathrm{KCl}$ is used for assem- 
bly. For fluorescence microscopy experiments the used ions and their respective concentration are shown in Table 3.3. In both cases, the protein is mixed in a reaction tube and incubated at $37^{\circ} \mathrm{C}$ for $16 \mathrm{~h}$ [3]. This is not practicable for the X-ray experiments and thus, the assembly for $\mathrm{X}$-ray experiments is performed at $21^{\circ} \mathrm{C}$.

Table 3.3: Overview of used ions in $2 \mathrm{mM}$ MOPS buffer at pH7.5 and their respective concentrations during microscopy measurements

\begin{tabular}{ll}
\hline Ion & Concentration $(\mathrm{mM})$ \\
\hline $\mathrm{NaCl}$ & 100 \\
$\mathrm{KCl}$ & 100 \\
$\mathrm{MgCl}_{2}$ & 4 \\
$\mathrm{CaCl}_{2}$ & 2 \\
Hexammine-cobalt(III) chloride & 0.05 \\
Spermine & 0.03 \\
\hline
\end{tabular}

\subsection{Static SAXS Experiments}

\subsubsection{In-house SAXS Setup}

All static SAXS experiments are performed in-house with the commercially available setup Xeuss 2.0 (Xenocs, Sassenage, France). A schematic representation of the setup and the beam path is shown in Fig. 3.1.

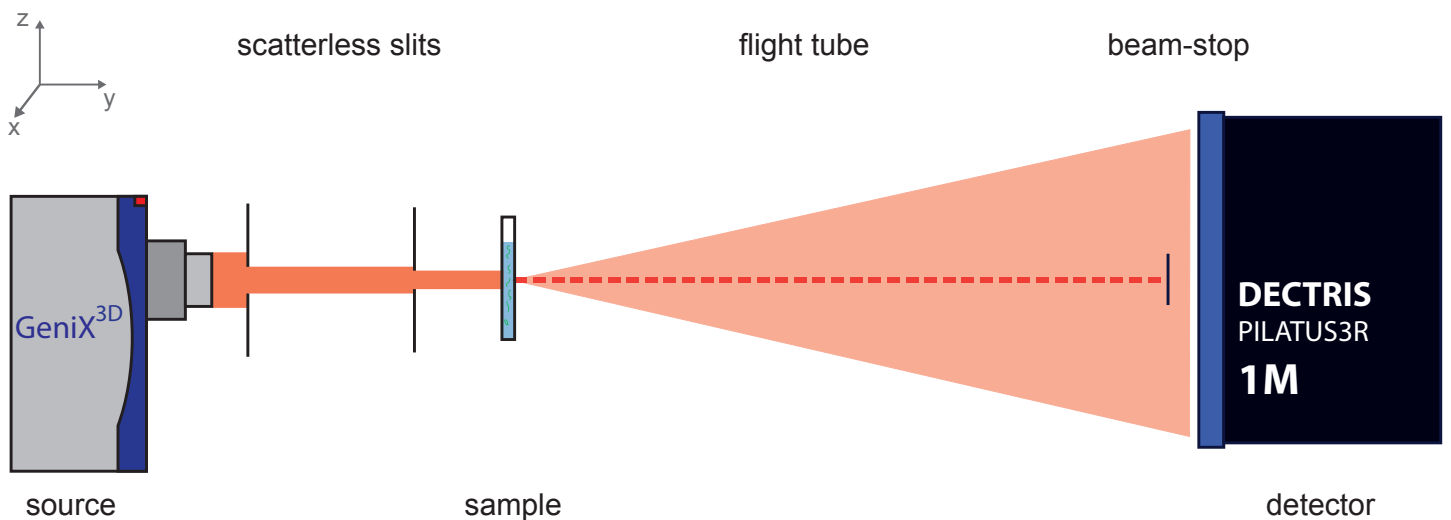

Figure 3.1: Scheme of the Xeuss 2.0 setup. X-rays are produced by the Cu K-alpha source and focused by mulitlayer optics. The final beam size is roughly $0.5 \mathrm{~mm} \times 0.5 \mathrm{~mm}$. Using scatterless slits, the beam is cleaned of parasitic scattering. After the sample along the $y$-axis a flight tube with a length of $2224 \mathrm{~mm}$ is placed. At the end of the flight tube the beam-stop is positioned to block the primary beam. Behind the flight tube a Pilatus3R $1 M$ detector is placed to record the data. 
The Xeuss 2.0 operates with a Cu K-alpha source $(\lambda=0.154 \mathrm{~nm})$ running at $50 \mathrm{keV}$ and $600 \mu \mathrm{A}$. The source has a parallel beam collimation to reduce smearing and multilayer optics to focus the beam. After the optics and the collimation mirror, two scatterless slits to clean the beam from parasitic scattering are located. Collimation optics and slits are adjusted to yield a beam size of approximately $0.5 \mathrm{~mm} \times 0.5 \mathrm{~mm}$. The collimation tube is under vacuum during all experiments. Next to the collimation tube the sample chamber is located. This chamber can either be in vacuum or in air. When measuring the sample in air, two Kapton windows are introduced between the evacuated collimation tube and the evacuated flight tube. For the buffer measurements, the sample chamber is filled with air (Chapter 4), whereas for the ion experiments (Chapter 5), the sample chamber is under vacuum. The flight tube, following the sample chamber, sets the distance between the sample and the detector. Depending on the distance between the sample and the detector, different length scales can be observed. The Xeuss 2.0 has three different flight tube sections, which can be inserted individually, giving access to different characteristic dimensions in real space (Table 3.4).

Table 3.4: Overview of the different sample to detector distances, the corresponding $q$-range and the respective real space dimensions. Adopted from introduction slides provided by Xenocs, Sassenage, France.

\begin{tabular}{lll}
\hline Sample to detector distance & $q$-range $\left(\mathrm{nm}^{-1}\right)$ & Real space dimension $(\mathrm{nm})$ \\
\hline 2485 & $0.042-2.21$ & $2.8-150$ \\
1190 & $0.085-4.58$ & $1.4-73$ \\
538 & $0.18-9.8$ & $0.64-34$ \\
360 & $0.27-14.2$ & $0.44-23$ \\
\hline
\end{tabular}

With that, a dimension in real space ranging from $0.44 \mathrm{~nm}$ to $150 \mathrm{~nm}$ can be detected. In the experiments shown here, a distance between the sample and detector of roughly $1220 \mathrm{~mm}$ is chosen, which gives an accessible $q$-range from around 0.07 to $4.8 \mathrm{~nm}^{-1}$ corresponding to a length scale of roughly $1.3 \mathrm{~nm}$ to $90 \mathrm{~nm}$ in real space. The beam-stop with a diameter of $3 \mathrm{~mm}$ is placed right before the tube exit window to block the primary beam. To collect the scattering data, a Pilatus3R 1M detector (981 x 1043 pixels, pixel size 172 x $172 \mu \mathrm{m}$, Dectris Ltd., Baden, Switzerland) is placed right after the exit window of the flight tube. For data aquisition, specfe (developed by Xenocs, Sassenage, France), a graphical interface version of spec is used.

\subsubsection{Calibration Measurements}

The Xeuss 2.0 is calibrated at least once per week. This is done to get the correct beam center and detector distance, as well as a scattering image to draw the mask for later analysis.

To calculate the beam center, the direct beam without the beam-stop is measured for $0.1 \mathrm{~s}$ (Fig. 3.2a and b). To estimate the beam center, a region around the beam is selected and 

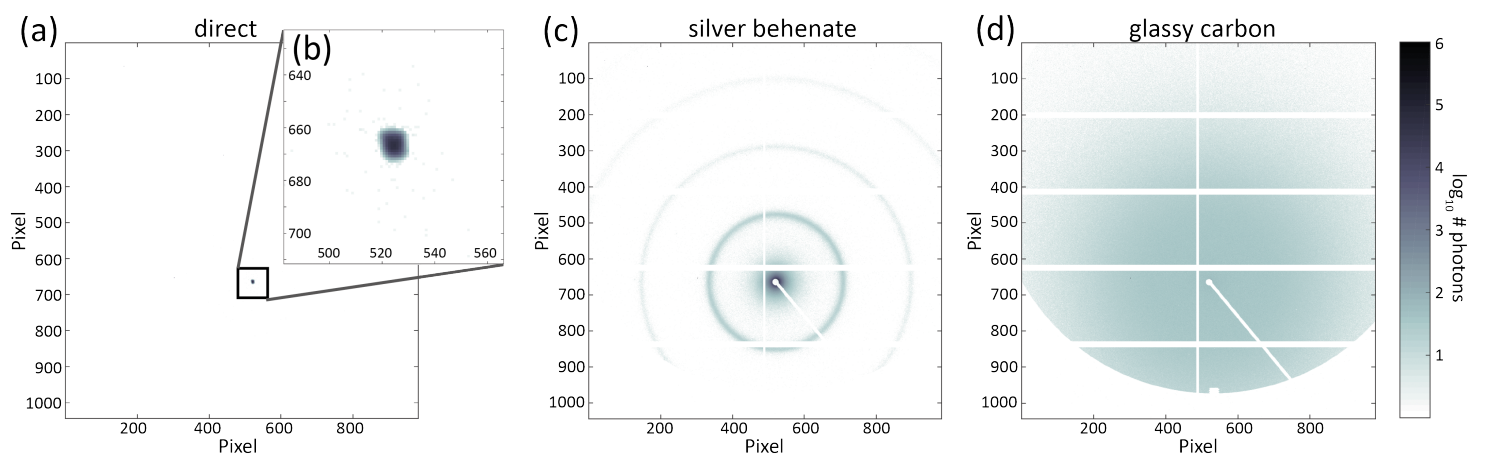

Figure 3.2: Scattering patterns of the calibration measurements. (a) Full detector image of direct beam measurement for $0.1 \mathrm{~s}$ to determine the beam center. (b) Detail of the direct beam measurement from (a). (c) Silver behenate measurement with an exposure time of $180 \mathrm{~s}$ to calculate the detector distance. (d) Scattering pattern of glassy carbon exposed for 180 s to draw the detector mask.

the center of mass calculated, using the software Foxtrot (developed by Xenocs and Solei Synchrotron, Paris, France). Afterwards the detector distance is determined using Silver behenate [5]. Silver behenate powder is placed between two Kapton sheets, which is placed in the setup and exposed to the X-rays for several minutes. Silver behenate has distinct well known diffraction peaks (Fig. 3.2c), by which the detector distance can be calculated. Peak positions are determined using a Gaussian fit to each peak in Foxtrot. As a third step, glassy carbon is exposed to X-rays for several minutes (Fig. 3.2d). The scattering pattern is used to draw the mask for later analysis. As a last step, the dark current, which is the signal on the detector without the X-ray shutter open, is recorded.

\subsubsection{Measurements}

After the calibration measurements, capillaries for the protein measurements are pre-measured with a caliper. Only capillaries with an outer diameter between 1.4 and $1.6 \mathrm{~mm}$ are used, as the diameter of the capillaries largely varies, even though they are, according to the manufacturer, all $1.5 \mathrm{~mm}$ in outer diameter (Fig. 3.3).

To reduce the influence of capillaries with different thicknesses, the same capillary is used for the buffer and the protein measurement. To obtain the thickness of the capillaries at the position where later the measurement will be performed, transmission scans of the empty capillaries are done. To avoid a strong oriented scattering signal from the capillary wall, the center of each capillary along the $x$-axis has to be found. The beam-stop is removed, the shutter opened and a scan along the $x$-axis is performed to retrieve the positions of the capillaries. Up to eight capillaries are placed in the sample holder and measured during one experiment. After the transmission scans of the empty capillaries are taken, the capillaries are removed from the 


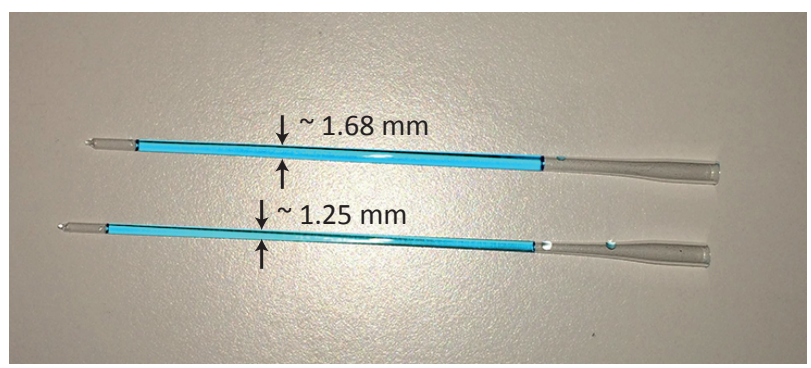

Figure 3.3: Variance of capillary diameters. Two capillaries are shown which have, according to the manufacturer, an outer diameter of $1.5 \mathrm{~mm}$. However, the thickness varies by $0.43 \mathrm{~mm}$ between the capillaries. For better visualization the capillaries are filled with blue food coloring.

setup and filled with ultrapure-water, sealed with wax and the positions of the capillaries are searched again by an $x$-axis scan. Transmission scans of the water-filled capillaries are taken. A direct beam without anything in the beam path is recorded additionally. With the transmission scans of the empty and water filled capillary, the thickness of the capillary can be calculated. For the buffer measurement, the wax from the capillary is removed by heating it up. The water is removed and buffer is filled into the capillary. The capillaries are again sealed, positions found and transmission scans taken. Test measurements of $60 \mathrm{~s}$ and $600 \mathrm{~s}$ are taken with the beam-stop in the beampath, to check if a strong oriented scattering signal (e.g. capillary walls) is visible. If not stated otherwise, 24 scattering patterns of $600 \mathrm{~s}$ exposure each are recorded, so that in the end the buffer and the sample, are each exposed for $4 \mathrm{~h}$. By dividing the $4 \mathrm{~h}$ in $10 \mathrm{~min}$ intervals, it can be checked if the sample suffers from radiation damage. After the scan is completed, transmission scans are recorded again. Now the capillaries are unsealed from wax once more, the buffer removed and protein is filled in the capillaries. A direct beam without anything in the beampath is taken. Again transmission scans before and after the protein measurement are performed. For the protein, the same procedure and exposure time as for the buffer is used. The transmission scans and the direct beams are needed to calculate the transmission values for normalizing the data. They are measured before and after each measurement to compare the values. If there is a large difference between both values, the primary intensity changed during the experiment and the data cannot be used for further analysis.

\subsubsection{Data Processing}

The first step in processing the data is drawing a mask form the glassy carbon scattering pattern. Dead pixels, the beam-stop and the detector grid are excluded. To check for radiation damage, an azimuthal integration for all scattering patterns of one capillary is performed using Mat- 
Lab2017a (The MathWorks, Natick, USA) and plotted together. The script is provided by Oliver Bunk (cSAXS beamline, SLS Switzerland). If no difference between the scattering profiles is observed the scattering patterns of the same sample are summed and an azimuthal integration is performed. The integrated scattering data are normalized to the transmission $T$ and exposure time $t$. The data from the buffer measurement is subtracted from the protein signal. The resulting scattering curve is normalized to the thickness of the capillary. For the experiments performed in Chapter 5 the data is further normalized to the CF for absolute scale. To get data on absolute scale $\left(\mathrm{cm}^{-1}\right)$ the CF needs to be calculated. This needs to be done only once for the operating machine. In this study, water is used as a primary standard to calculate the CF. An empty capillary is measured for $24 \mathrm{~h}$ and afterwards the water-filled capillary is measured for $24 \mathrm{~h}$ as well. Transmission scans of both, the empty and the water-filled capillary are taken before and after the measurement. In Appendix A the calculation of the CF for the setup in full vacuum is shown. The thickness of the capillary is calculated using the following formula:

$$
x_{w}=\frac{1}{\mu / \rho_{w}} \frac{1}{\rho_{w}} \ln \left(\frac{T_{c+w}}{T_{c}}\right),
$$

where $\mu / \rho_{w}=10.37 \mathrm{~cm}^{2} \mathrm{~g}^{-1}$ taken from

https://physics.nist.gov/PhysRefData/XrayMassCoef/ComTab/water.html is the mass attenuation coefficient for an energy of $8 \mathrm{keV}$ and a density $\rho=1 \mathrm{~g} \mathrm{~cm}^{-3} . T_{c}$ is the transmission through the empty capillary and $T_{c+w}$ is the transmission for the water-filled capillary. After normalization, the data is analyzed with the software package PRIMUS [6] from EMBL (ATSAS, EMBL, Hamburg, Germany) and self written scripts. For the different analyzing methods, a monodispers sample in solution is considered. A Guinier analysis for elongated rods is performed by fitting the small $q$-values. The fitting range is adjusted such that $q R_{c} \leq 1.3$. A drawback of the Guinier analysis is that only the beginning of the curve is taken into account which means only a small part of the data set is used for this analysis. For analysis of the ion experiments (Chapter 5) a polynomial is fitted to the scattering profiles and the first and second derivative are calculated. The mean steepness (first derivative) of the whole curve and the mean curvature (second derivative) in a range from $0.2-0.5 \mathrm{~nm}^{-1}$ are calculated. Furthermore, the data is fitted to a model which was used by Brennich et al. [4] and Hémonnot et al. [7] for vimentin and keratin, respectively. The model is based on a micelle model introduced by Pedersen [8]. The radial electron density $\rho_{e}$ of the filaments as a solid core, surrounded by a cloud of flexible Gaussian chains is modeled (Fig.3.4). The Gaussian chains correspond to the C-terminal regions (tails) protruding from the filament $[9,10]$.

The form factor of micelles is described as: 
(a)

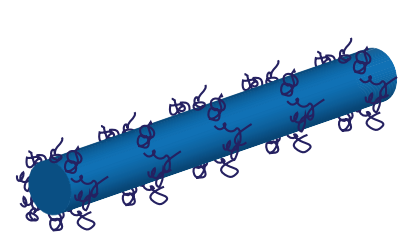

(b)

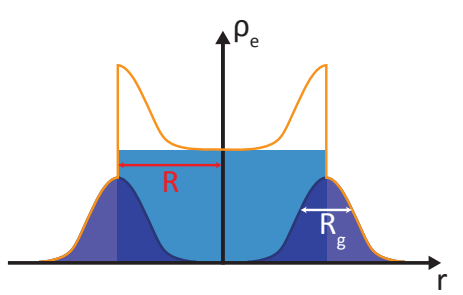

Figure 3.4: Description of the model used for fitting the data.(a) Sketch of a vimentin filament. The rod regions form the cylindrical core (light blue) and the tail regions (dark blue) protruding from the filament form a cloud of Gaussian chains around the core. (b) The radial electron density of the model for vimentin. The core cylinder with the radius $R$ corresponds to the light blue part, and the Gaussian chains with a radius of gyration $R_{g}$ refer to the dark blue parts. The total electron density is displayed by the orange line. Adapted from [4].

$$
F(q)=\beta\left[F_{s}(q)+\lambda b^{2} F_{c}(q)+2 b S_{s c}(q)+b^{2} S_{c c}(q)\right],
$$

where $\beta \propto \beta_{s}^{2}$ is the total scattering from the core per length, $\beta_{s}$ is the forward scattering of the core per ULF, $\lambda$ denotes to the average distance between the tails, $b$ refers to the ratio between the scattering from the cloud of tails to the scattering from the core $\left(b=\beta_{c} / \beta_{s}\right)$. The four terms define the self correlation term of the core $\left(F_{s}\right)$, the self correlation of the chains $\left(F_{c}\right)$ and the cross-term between the core and chains $\left(S_{s c}\right)$, as well as the cross-term between different chains $\left(S_{c c}\right)$. To model vimentin filaments the following assumptions have been made: (I) One ULF is approximately $43 \mathrm{~nm}$ in length $(l)$ and has 32 monomers, corresponding to 32 tails protruding from the filament $(n)$ and therefore, $\lambda=l / n=1.34 \mathrm{~nm}$. (II) The Gaussian chains are situated at the rod surface and (III) the persistence length $(0.3-2 \mu \mathrm{m}$ [11]) of the filament is larger than the accessible length scales during the measurement. The model described above is extended by a term that describes the vimentin tetramers. It was shown that when assembling vimentin at low ion concentrations, tetramers are still found in the solution [12]. To account for this, the form factor is extended as already done by Brennich et al. [4]. The tetrameric term is not described by a model, but the scattering profile of vimentin tetramers is used. Using a least square fitting, the optimal fit is found [13].

\subsection{Microscopy Experiments}

To check, whether single filaments or networks of vimentin are formed AFM and fluorescence microscopy experiments are performed additionally to the SAXS measurements. 


\subsubsection{Atomic Force Microscopy}

For AFM measurements, dried vimentin on mica sheets are prepared. First, a mica sheet is glued to a glass slide using UV-curable adhesive NOA81, and cured for $30 \mathrm{~min}$ by UV-radiation (365 nm, 2 x 8 W; Herolab GmbH, Wiesloch, Germany). The mica sheets are cleaned using sticky tape. Assembled vimentin is diluted 1:5 in buffer, mixed 1:1 with $0.25 \%$ glutaraldehyde diluted with the same buffer as the assembled vimentin is stored (e.g. $100 \mathrm{mM} \mathrm{KCl} \mathrm{in} 2 \mathrm{mM} \mathrm{PB}$ ) and incubated for $30 \mathrm{~s}$ to fix the filaments. After fixation of the sample, $50 \mu \mathrm{L}$ are added to the mica substrate and incubated for $1 \mathrm{~min}$ before rinsing everything thoroughly with water. Mica sheets are dried using nitrogen gas and are stored until use in petri dishes sealed with parafilm. Measurements are performed with an MFP-3D Infinity AFM (Asylum Research, Oxford Instruments, Abingdon, U.K.) equipped with a micro cantilever (resonant frequency of $70 \mathrm{kHz}$ and a spring constant of $2 \mathrm{~N} / \mathrm{m}$, Olympus, Tokyo, Japan). All images are analyzed with the open source software Gwyddion (http://gwyddion.net/).

\subsubsection{Fluorescence Microscopy}

An inverted FluoView IX81 confocal microscope (Olympus, Tokyo, Japan) is used for fluorescence microscopy, equipped with a 60X UplanSApo oil objective (numerical aperture of 1.45, Olympus, Tokyo, Japan). To detect the labeled vimentin, a laser with an excitation wavelength of $635 \mathrm{~nm}$ is used and the signal is recorded. In total, $10 \mu \mathrm{L}$ of the protein solution is added between two cover slips and directly imaged. All images are obtained at room temperature and analyzed with the open source software ImageJ (https://imagej.nih.gov/ij/).

\subsection{Time-Resolved SAXS Experiments}

Time-resolved SAXS experiments require a high flux and a small beam. Therefore, these experiments are performed at two different synchrotrons. At the ID13 beamline of the European Synchrotron Radiation Facility (ESRF) in Grenoble, France and at the cSAXS beamline of the Swiss Light Source (SLS, Villigen, Switzerland) experiments are performed.

\subsubsection{Finite Element Method Simulations}

A time-resolved observation of vimentin assembly will be investigated. This is achieved using microfluidics. For experiments a 5-inlet and 1-outlet geometry is chosen (Fig.3.5 a). Through the central inlet the protein is pumped into the system. Through two sheath inlets, buffer without assembly reagents is pumped to shield the protein from the assembly buffer until the pro- 
tein stream is focused [14]. Via the side inlets the assembly buffer is injected. By mixing of the assembly buffer with the protein the assembly starts. By investigating different positions in the channel, the different assembly states can be observed. Flow Simulations for the protein in the microfluidic channels are performed using COMSOL Multiphysics 5.2a (COMSOL AB, Stockholm, Sweden).
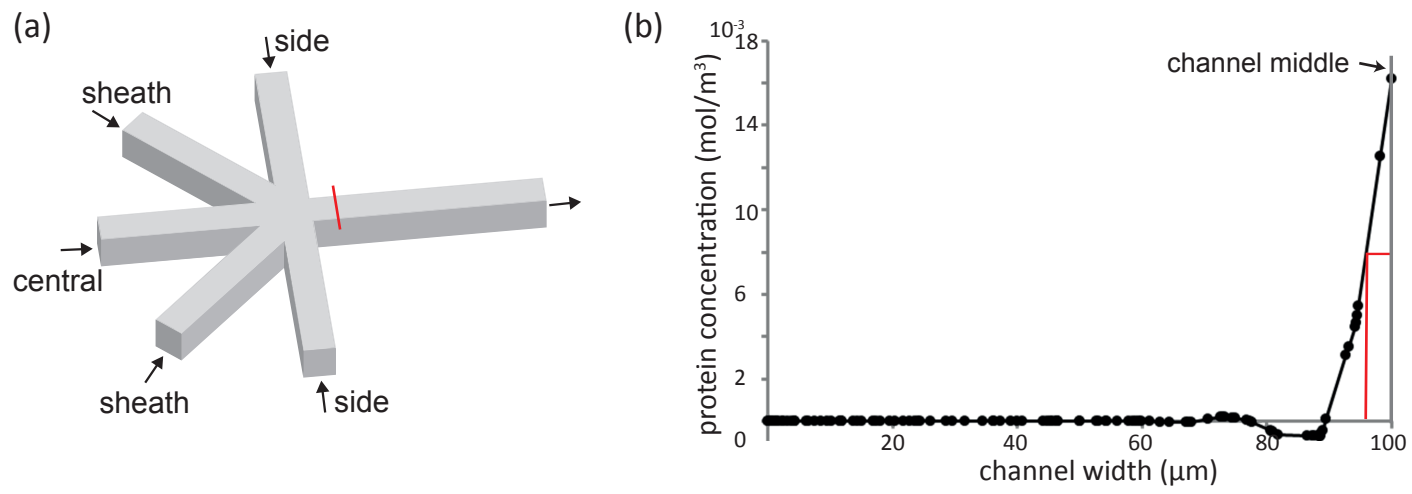

Figure 3.5: Device geometry and protein stream width. (a) Sketch of the device geometry used during the timeresolved measurements. The device consists of five inlets: central (protein/colloids), two sheath (buffer), two side inlets (assembly buffer/buffer) and one outlet. (b) The protein concentration at the position of the red line in (a). A stream width of roughly $10 \mu \mathrm{m}$ (full width half maximum) is achieved with the flow rates used.

The 3D geometry of the desired microfluidic channels is designed in the software. The channels are $200 \mu \mathrm{m}$ wide and $160 \mu \mathrm{m}$ high. To reduce the computational time and due to symmetry in the device only half the device is simulated. A physics controlled adaptive mesh size is used for the simulations, with mesh sizes ranging from 0.07 to $4.5 \mu \mathrm{m}$. All simulations are performed with no-slip boundary conditions at the channel walls and laminar flow. Diffusion constants for $\mathrm{KCl}$ and vimentin are $D_{K C l}=1.84 \cdot 10^{-9} \mathrm{~m}^{2} / \mathrm{s}$ and $D_{v i m}=2.2 \cdot 10^{-11} \mathrm{~m}^{2} / \mathrm{s}$. During protein assembly, the diffusion constant of vimentin decreases, however this is not included in the simulation, as viscometry measurements showed that the viscosity only increases by a small amount during the first seconds of vimentin assembly $[15,16]$. Therefore, the diffusion constant of tetrameric vimentin is used for the whole simulation. For the experiments, a fast mixing of protein with the assembly buffer is desired. This can be achieved by a narrow protein stream width. Here, a stream width of roughly $10 \mu \mathrm{m}$ is chosen, as the diffusion of the salt of the assembly buffer into the protein steam is on the order of $\mathrm{ms}$. To gain a $10 \mu \mathrm{m}$ protein stream flow rates are adjusted to $150 \mu \mathrm{L} / \mathrm{h}$ for the side inlet, $7.5 \mu \mathrm{L} / \mathrm{h}$ for the sheath inlet and $15 \mu \mathrm{L} / \mathrm{h}$ for the central inlet (Fig.3.5 b). The small negative dip in the simulation at around $80-90 \mu \mathrm{m}$ is probably due to a coarser mesh size at this position and computational errors (Fig. 3.5b). 


\subsubsection{Preparation of Microfluidic Channels}

For the experiments, two different types of devices are used. One type consists of two Kapton sheets ( $8 \mu \mathrm{m}$ thick) glued together with an UV-curable adhesive NOA81. A similar protocol is described in [17]. The other device type is solely made out of Cyclic Olefin Copolymer (COC). A detailed description on how to prepare COC devices can be found in Chapter 6 [18].

\section{Device Master}

For the preparation of the microfluidic devices, a device master is needed, which is produced by Gerrit Brehm. The device master had a height of $160 \mu \mathrm{m}$ to $200 \mu \mathrm{m}$ for the performed experiments. Channel structures are produced on a 2-inch silicon wafer (Si-wafer; MicroChemicals, Ulm, Germany).

(a)

Si-wafer

(b)

Si-wafer with resist

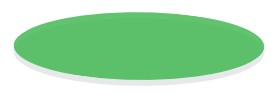

(c)
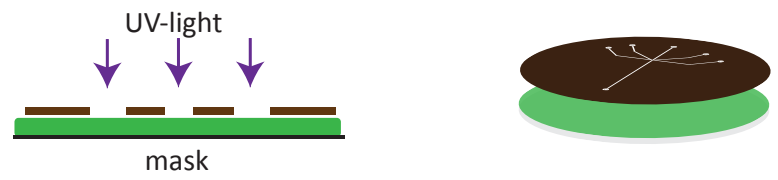

(d)
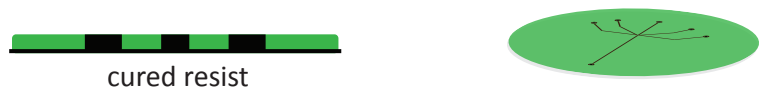

(e)
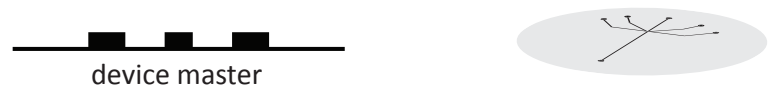

Figure 3.6: Manufacturing steps of the device master. (a) A clean Si-wafer is used and (b) SU-8 2150 is spin coated on the wafer. (c) A photo mask with the device geometry is aligned with the wafer with resist and (d) the resist is cured with UV-light at the exposed places. (e) After removing the non-cured resist the wafer with the microfluidic channels is finished.

The channel structure is transferred from a mask onto the Si-wafer using soft photolithography methods. A Si-wafer (Fig. 3.6a) is rinsed with 2-propanol and dried for $20 \mathrm{~min}$ at $200^{\circ} \mathrm{C}$. Afterwards the Si-wafer is coated by spincoating using $2 \mathrm{~mL}$ of SU-8 2150 (SU-8, MicroChem, Newton, USA) in three steps (Fig. 3.6b). In the first step, the wafer is spun for $30 \mathrm{~s}$ at $500 \mathrm{rpm}$ with an acceleration of $100 \mathrm{rpm} / \mathrm{s}$ on a spincoater (Optispin SB20, ATMgroup, Salem/Beuren, Germany). In the second spinning step the wafer is spun again for $30 \mathrm{~s}$ at $1250 \mathrm{rpm}$ and an acceleration of $200 \mathrm{rpm} / \mathrm{s}$. In the last step the speed is increased to $2000 \mathrm{rpm}$ with an acceleration 
of $300 \mathrm{rpm} / \mathrm{s}$ and a duration of $60 \mathrm{~s}$. The spincoating is followed by a soft bake step for initially $7 \mathrm{~min}$ at $65^{\circ} \mathrm{C}$. Next, the wafer is transferred to a hot plate at $95^{\circ} \mathrm{C}$ for $30 \mathrm{~min}$. As the SU-8 2150 is very viscous accumulated resist at the edge of the wafer is clearly visible. An edge bead removal is performed, as suggested by the resist provider. After the soft bake step the edge bead removal is performed. The wafer is spun with a spincoater, while developer solvent (mr-Dev 600, microresist technology, Berlin, Germany) is carefully dropped on the edges of the wafer to remove the excess SU-8 2150. The spinning for the edge bead removal is performed in two steps. For the first $15 \mathrm{~s}$ a speed of $500 \mathrm{rpm}$ and an acceleration of $100 \mathrm{rpm} / \mathrm{s}$ is used. In the second step the speed is increased to $2000 \mathrm{rpm}$ for $30 \mathrm{~s}$ with an acceleration of $300 \mathrm{rpm} / \mathrm{s}$. After the edge bead removal, the wafer is baked for $25 \mathrm{~min}$ at $95^{\circ} \mathrm{C}$. With the mask aligner (MJB4, SÜSS MicroTec AG, Garching, Germany) a mask (Selba S.A.,Versoix, Switzerland) with the desired channel structure and the wafer are brought in position and exposed to UV-light (Fig. 3.6c). The exposure is performed at $22 \mathrm{~mW} / \mathrm{cm}^{2}$ for $20 \mathrm{~s}$ with a laser wavelength of $365 \mathrm{~nm}$. A post exposure bake follows to cross-link the UV-light exposed parts in two steps. Initially for $5 \mathrm{~min}$ at $65^{\circ} \mathrm{C}$ and afterwards for $20 \mathrm{~min}$ at $95^{\circ} \mathrm{C}$ (Fig. 3.6d). Finally, the non-exposed SU-8 2150 is removed using the SU-8 developer. The wafer is placed into a beaker filled with the developer and incubated for $20 \mathrm{~min}$. Gentle shaking of the beaker helps to remove the resist. After $20 \mathrm{~min}$ the wafer is taken out of the beaker, rinsed first with acetone, afterwards with 2-propanol and finally with ultrapure-water and dried with nitrogen. A final hard bake step for $5 \mathrm{~min}$ at $150^{\circ} \mathrm{C}$ is performed. In a very last step, the wafer is coated with fluorosilane ((heptafluoropropyl)trimethylsilane) for at least $1 \mathrm{~h}$ (Fig. 3.6e), so that lateron PDMS can be peeled of the wafer more easily.

\section{UV-curable adhesive Kapton Devices}

The protocol for preparation of UV-curable adhesive/Kapton devices is adapted from [17]. For the UV-curable adhesive/Kapton devices two $8 \mu$ m thick Kapton foils are used. First the device master is cleaned from dust particles (Fig. 3.7a) using 2-propanol. A PDMS stamp is prepared from the device master (Fig. 3.7b). PDMS and a Sylgard 184 cross-linker are mixed at a ratio of 10:1 and the PDMS is desiccated for 15 min to remove air bubbles. PDMS is poured on the device master and desiccated again for $15 \mathrm{~min}$ so that all air bubbles are removed. The PDMS is then hardened for $2 \mathrm{~h}$ at $65^{\circ} \mathrm{C}$. The PDMS is cut with a scalpel and carefully removed from the device master using 2-propanol. A second PDMS stamp is fabricated from the first one (Fig. 3.7c). The edge of the first PDMS stamp is colored with permanent marker. The PDMS stamp is placed into the plasma cleaner (PDC-32GHarrick Plasma, Ithaca, NY, USA) which is connected to a vacuum pump. Air is extracted from the plasma cleaner until a plasma with a purple-pink shade is visible. After $30 \mathrm{~s}$ of plasma activation the PDMS stamp is coated with 
$70 \mu$ l hexamethyldisilazane (HMDS) for 10 min in a desiccator. By coating of the stamp with HMDS, the second PDMS stamp can be detached more easily from the fist one. Desiccated and air-bubble free PDMS is poured onto the PDMS stamp and it is desiccated for $15 \mathrm{~min}$. PDMS is hardened for $2 \mathrm{~h}$ at $65^{\circ} \mathrm{C}$. After the PDMS is hardened, the PDMS is cut inside the colored edge of the first stamp. As the first and second PDMS stamp are still bound together, both are cut out. Both PDMS stamps are carefully detached from each other. Each stamp is only used once, as the channel structure may have tiny cracks. UV-curable adhesive NOA81 is pipetted on the channel structure and desiccated for $15 \mathrm{~min}$ to remove air bubbles from the structure (Fig. 3.7 d). In the meantime, an aluminum block is cleaned with 2-propanol. A Kapton foil is placed on the aluminum block and the foil is flattened as thoroughly as possible. UV-curable adhesive NOA81 is put on the Kapton foil (Fig. 3.7e) and the PDMS stamp is pressed on top and all air bubbles are pressed out. Under the UV-lamp (365 nm, 2 x 8 W; Herolab GmbH, Wiesloch, Germany) the UV-curable adhesive is partially cured for $3 \mathrm{~min}$ (Fig. 3.7f). Afterwards the holes for the inlet and outlet are made using a biopsy puncher with a diameter of $0.75 \mathrm{~mm}$ (Harris UniCoreTM puncher, Plano, Wetzlar, Germany)(Fig. 3.7g). The PDMS stamp is carefully removed and the excessed UV-curable adhesive NOA81 at the edges is cut away (Fig. $3.7 \mathrm{~h}$ ). On a second aluminum block, a second Kapton foil is prepared as described before. The partially cured UVcurable adhesive is pressed on the second Kapton foil, while wrinkles are to be avoided. The device is cured for at least $1 \mathrm{~h}$ under the UV-lamp (Fig. 3.7i) and finally cut to fit into the sample holder (Fig. 3.7j). 
(a)
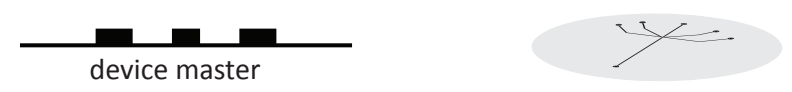

(b)
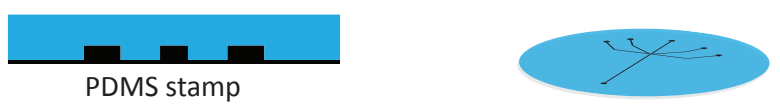

(c)
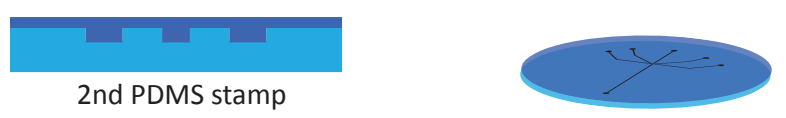

(d)

(e)
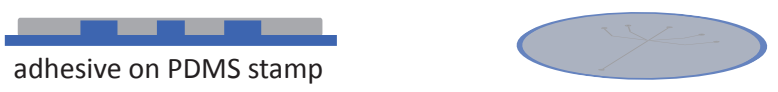

adhesive on Kapton foil

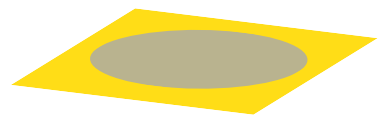

(f)

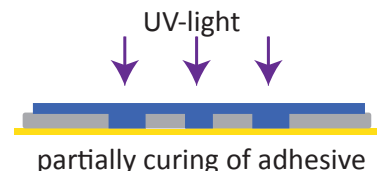

(g)

(h)
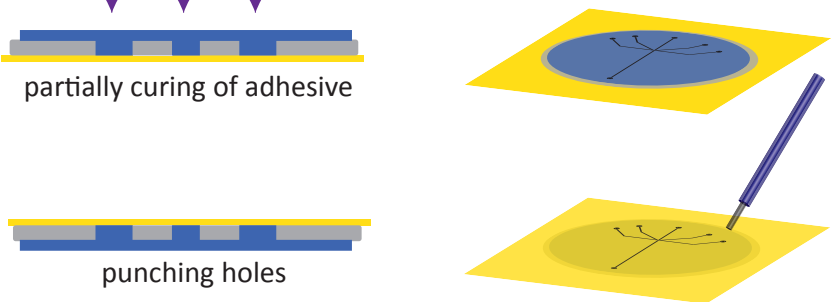

half finished device

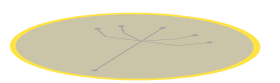

(i)

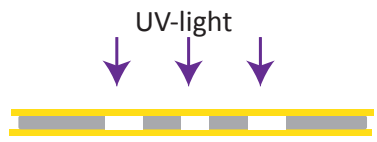

(j)

fully curing of adhesive

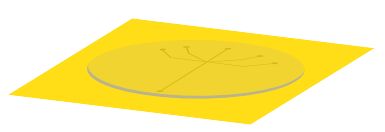

finished device

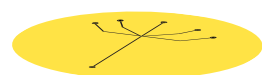

Figure 3.7: Manufacturing of UV-curable adhesive/Kapton devices. (a) The device master is cleaned from dust. (b) A PDMS copy (blue) of the channel structure is produced. (c) A second PDMS-stamp is produced by plasma activating the first stamp and coating it with HMDS. (d) UV-curable adhesive is placed on the second PDMS stamp and desiccated for 15 min to remove air bubbles. (e) UV-curable adhesive is dropped on the Kapton foil. (f) PDMS stamp and Kapton foil, both with UV-curable adhesive, are brought together and the UV-curable adhesive is partially cured for 3 min with UV-light. (g) Holes are punched for the in-and outlets of the channel. (h) The PDMS stamp is removed. (i) A second layer of Kapton foil is placed on the partially cured UV-curable adhesive and fully cured with UV-light. (j) The device is cut in a final step. 


\subsubsection{Experimental Setup at ESRF - ID13 Beamline}

Experiments are carried out at the experimental hutch II of the ID13 beamline of the ESRF. A schematic representation of the setup is shown in Fig. 3.8. The beam is produced by electrons which are additionally accelerated by a vacuum undulator. Using a liquid nitrogen cooled double-crystal $\mathrm{Si}(111)$ monochromator, an energy of $13.9 \mathrm{keV}$ is selected and first beam focusing is performed. The small beam size is achieved using 54 beryllium compound refractive lenses (Be-CRL) [19]. For micro-SAXS conditions, three additional apertures are in the beam path. Measurements at the ID13 beamline are carried out at a primary beam intensity of $8 \cdot 10^{11}$ photons per second. The beam size during the experiment is $2.7 \times 1.7 \mu \mathrm{m}^{2}$. The sample in the sample holder is mounted on a scanning stage. Using a light microscope that can be moved in the X-ray path, the microfluidic channels can be brought into focus of the beam. A $7 \mathrm{~cm}$ long helium filled flight cone with a polypropylene window is placed after the sample to reduce air scattering. A beam-stop, which is a few $\mathrm{mm}$ in length and roughly $300 \mu \mathrm{m}$ in diameter, is placed to block the primary beam. Finally the signal is recorded at a sample to detector distance of $\sim 0.95 \mathrm{~m}$ on an Eiger 4M detector $\left(2070\right.$ x 2167 pixels, pixel size $75 \times 75 \mu \mathrm{m}^{2}$, Dectris Ltd., Baden, Switzerland).

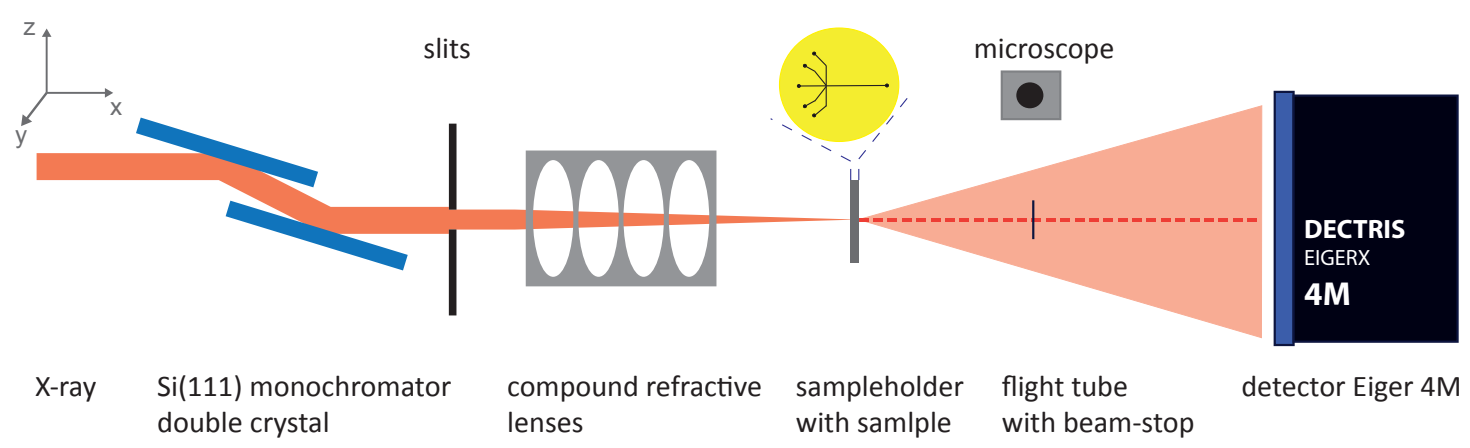

Figure 3.8: Scheme of the experimental hutch II of the ID13 beamline at the ESRF. The monochromatized beam is focused by beryllium compound refractive lenses. The sample is placed at the focal spot with a beam size of roughly $2.7 \times 1.7 \mu \mathrm{m}^{2}$. With a microscope, which can be moved in the beam path, the sample positions can be chosen. A beam-stop right after the the $7 \mathrm{~cm}$ long flight tube blocks the primary beam. Data is recorded on the detector.

\subsubsection{Experimental Setup at SLS - cSAXS Beamline}

At the SLS in Swizerland, the experiments are conducted at the cSAXS beamline. A schematic representation of the cSAXS beamline is shown in Fig 3.9. The beam is produced by accelerated electrons, which get accelerated additionally with an undulator source. The energy of $11.2 \mathrm{keV}$ is selected using a double-crystal $\mathrm{Si}(111)$ monochromator. Furthermore, the monochromator 
is used for focusing of the beam in horizontal direction. A bendable mirror is used for focusing the beam in vertical direction. Slits before the monochromator and after the bendable mirror are used to clean the beam from parasitic scattering. Slits with a horizontal aperture are used to optimize the beam size, however reduce the flux by around $50 \%$. The beam intensity is $2 \cdot 10^{11}$ photons per second. At the focal point where the measurements take place, the beam size is about $12 \times 29 \mu \mathrm{m}^{2}$. The position of the microfludic channels can be observed with a microscope, to which the sample can be moved without changing the $z$ position. Directly behind the sample, a $7 \mathrm{~m}$ long evacuated flight tube is located to reduce parasitic air scattering. A beam-stop is located at the end of the flight tube to block the primary beam. At a sample to detector distance of $7.087 \mathrm{~m}$ a Pilatus 2M detector (1475 x 1679 pixels, pixel size: $172 \times 172 \mu \mathrm{m}^{2}$, SLS detector group, Villigen Switzerland) [20] is placed to record the data.

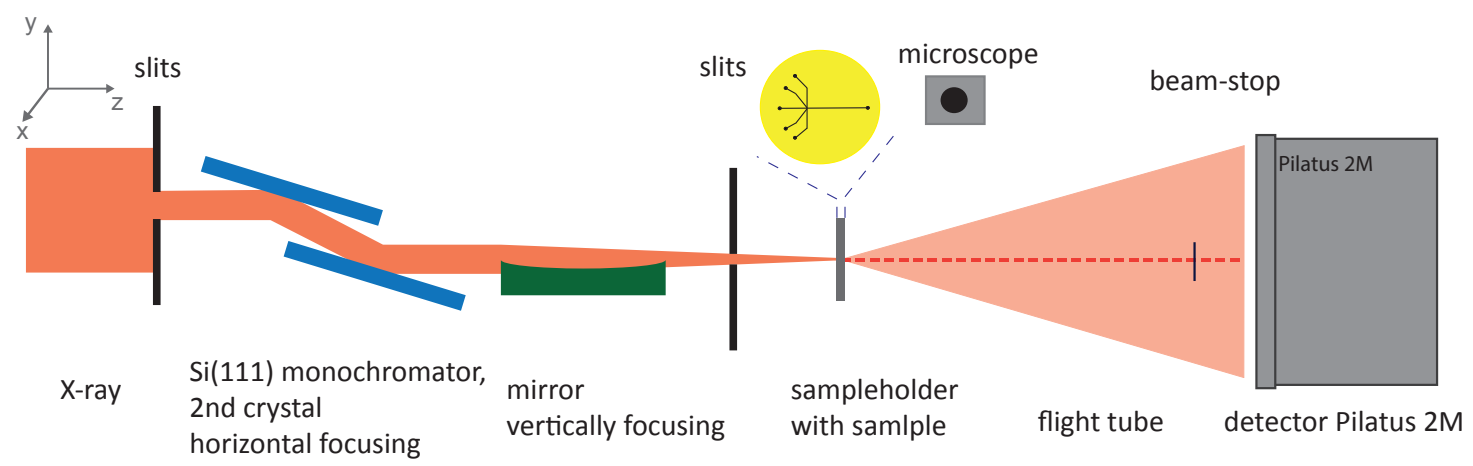

Figure 3.9: Representation of the CSAXS beamline at the SLS. The monochromatized beam is focused by slits and a beandable mirror. The sample is placed in the focal spot with a beam size of about $12 \times 29 \mu \mathrm{m}^{2}$. The sample positions could be chosen, with a microscope at the beamline. A beam-stop right after the $7 \mathrm{~m}$ long evacuated flight tube and before the detector blocks the primary beam intensity. Data is recorded on the detector

\subsubsection{Sample Preparation and Measurement at the Beamline}

At the synchrotron, all buffers and the protein solution are degassed prior to usage. Polyethylene tubing (inner diameter $0.38 \mathrm{~mm}$, outer diameter $1.09 \mathrm{~mm}$, Intramedic Clay Adams Brand, Becton Dickinson and Company, Sparks, USA) is connected to the sample holder (Fig 3.10). 
(a)

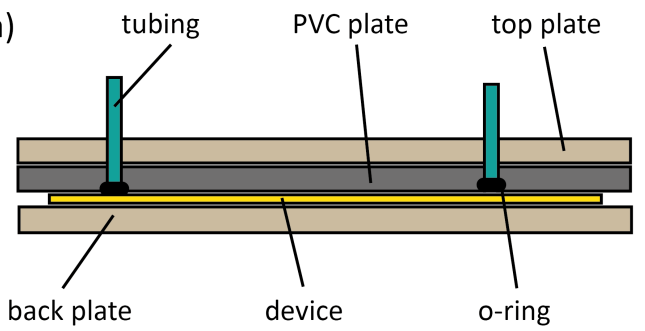

(b)

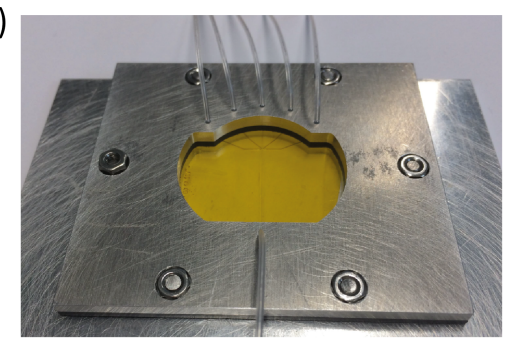

Figure 3.10: Sample holder for the microfluidic devices. (a) Scheme of the sample holder, consisting of a metal back and top plate and a PVC plate with indention for the o-rings. The back plate and the PVC plate sandwich the device. Tubing is threaded through the top and PVC plate and made leak-tight by the o-rings. (b) Picture of the sample holder with a UV-curable/adhesive device ready to measure.

The sample holder based on one shown in Urbani's dissertation [21] and developed further by Gerrit Brehm, consists of two metal plates that sandwich a polyvinylchloride (PVC) plate and the device (Fig. 3.10a). The plates contain a window for the measurements. The front metal plate and the PVC plate contain small holes for the tubing. Tubing is threaded through the holes. Small o-rings, which are placed in intentions in the PVC plate, make the sample holder leak-tight. The device is aligned with the tubing on the PVC plate and fixed with sticky tape. The construction is fixed with screws. The metal back-plate is larger than the other plates, as it serves as an adapter to the beamline sample stage.

The device is filled with buffer, and the ends of the tubing are sealed after the complete device and tubing is filled with buffer and no air bubbles are inside the system. Hamilton Gastight glass syringes (Bonaduz, Switzerland) with $1 \mathrm{~mL}$ for side inlets and $0.5 \mathrm{~mL}$ syringes for the sheath and central inlet are used. In protein experiments the syringes for the side inlets contained the assembly buffer ( $2 \mathrm{mM} \mathrm{PB}, 100 \mathrm{mM} \mathrm{KCl}, \mathrm{pH} 7.5)$, for the sheath inlet only buffer (2 mM PB, pH 7.5) and the central inlet the protein solution (vimentin wt in $2 \mathrm{mM} \mathrm{PB}, \mathrm{pH} 7.5$ ) is filled in the syringe. For colloid experiments the syringes for the side and sheath inlets are filled with phosphatebuffered saline (PBS) and the central syringe contains the colloids in PBS. The syringes are connected to the syringe pumps (neMESYS, Cetoni GmbH, Korbußen, Germany), which allowed a precise flow control. To set up a measurement at the beamline, the outlet tubing is opened. First, one sheath buffer syringe is started with a high flow rate. The tubing is cut open and the syringe connected to the tubing, introducing a small air bubble to the system. This is done to see where the content of the syringe is at the moment, as the whole device is filled with buffer. For the second sheath buffer the procedure is repeated. As soon as both air bubbles exit from the outlet the flow speed is decreased to about $200 \mu \mathrm{L} / \mathrm{h}$. Afterwards the side inlets are started at a high flow rate with again introducing a small air bubble. As soon as the air bubble is near the device, the speed is reduced to $100 \mu \mathrm{L} / \mathrm{h}$. The same is repeated for the second side inlet. After that, the side inlets are reduced to $50 \mu \mathrm{L} / \mathrm{h}$, whereas the sheath buffer inlets are kept constant 
at $200 \mu \mathrm{L} / \mathrm{h}$. At last, the central inlet is started in the same way as the previous inlets. As soon as the air bubble entered the tubing, the flow rates of all inlets are slowly reduced to the final flow rates of $150 \mu \mathrm{L} / \mathrm{h}$ and $7.5 \mu \mathrm{L} / \mathrm{h}$ for the side and sheath inlets respectively and $15 \mu \mathrm{L} / \mathrm{h}$ for the central inlet. The buffer measurement is performed. First the cross-section of the device is located. From this position a mesh can be defined where the measurement is performed. The mesh is defined in both directions, so that the tetrameric signal from the protein, as well as the signal from the assembling protein can be detected. The width of the mesh is larger than the central channel, so that the whole channel width can be detected (Fig. 3.11).

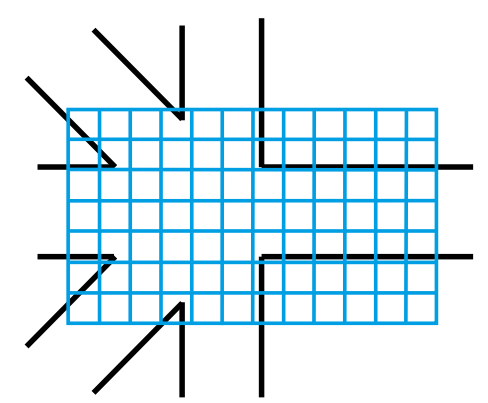

Figure 3.11: Schematic representation of the mesh used in synchrotron experiments. The device geometry is outlined in black, with a representation of the mesh (light blue) as used in synchrotron experiments. Note that the mesh size during the experiments is smaller than indicated here. The mesh has a larger size than the actual channel width to ensure that the whole channel is measured.

A microscopy image of the region of interest is taken before and after the X-ray scan. After the measurement, the central and the sheath inlets are increased in velocity until the air bubble of the central inlet is near the device. The air bubble is the border between buffer and gold colloids/protein. Velocities are reduced to $50 \mu \mathrm{L} / \mathrm{h}$. As soon as all air bubbles are gone, the velocities are set to the same velocities as for the buffer measurement. The same mesh is taken for the protein measurement as it is defined for the buffer measurement. During the scan, a darkfield image is calculated. The sum over the whole scattering pattern is taken. With this method it can be observed if clogging of the protein occurred or if an air bubble has formed in the channel. At ID13 each position is exposed for $1 \mathrm{~s}$. For measurements at the cSAXS beamline $5 \mathrm{x} 1 \mathrm{~s}$ exposure is chosen for each position.

\subsubsection{Data Processing}

For data processing, a mask is drawn and an azimuthal integration is performed. Background subtraction is performed by subtracting the buffer signals from exactly the same position as the protein signal. By doing so, an unevenness of the devices can be overcome, which would lead to a false signal. The channels do not have the same height over the whole length of the device. 
Thus, to minimize the different sample heights, the buffer signal recorded at the same position as the protein signal is subtracted.

After azimuthal integration, the scattering profiles of the colloid data are analyzed the following: First a Guinier analysis of the small $q$-values using Equation 2.25 is performed with the software package PRIMUS (ATSAS, EMBL, Hamburg, Germany). As a limit for Guinier analysis $q R_{g} \leq 1.3$ is used. Guinier analysis for the data taken at ID13 could not be performed, as the $q$-range did not include the low $q$-values needed. In a next step the pddf $p(r)$ is calculated with Equation 2.29 to validate the results from the Guinier analysis. This analysis is also performed using PRIMUS (ATSAS, EMBL, Hamburg, Germany). In a last step the form factor $P(q, R)$ for solid spheres is fitted to the data using Matlab2017a (The MathWorks, Natick, MA, USA). The form factor for solid spheres can be written as follows:

$$
P(q, R)=\left[\frac{3 \sin (q R)-q R \cos (q R)}{q R^{3}}\right]^{2} .
$$

Data from the protein measurements could not be analyzed sufficiently due to clogging of the protein in the channel and therefore, those experiments are only used as a proof of principle. Scattering profiles of only four positions could be extracted. With these four positions, the assembly process could not be deciphered in detail. 


\subsection{Materials}

Table 3.5: Used chemicals and the respective company

\begin{tabular}{|c|c|}
\hline Chemical & Company \\
\hline $1.5 \mathrm{~mm}$ quartz glass capillaries & Hilgenberg, Malsfeld, Germany \\
\hline 2-inch silicon wafer & MicroChemicals, Ulm, Germany \\
\hline $50 \mathrm{kDa}$ cut-off Tubing & $\begin{array}{l}\text { SpectraPor, Carl-Roth GmbH, Karlsruhe, Ger- } \\
\text { many }\end{array}$ \\
\hline ATTO647N-maleimide & ATTO-TEC GmbH, Siegen, Germany \\
\hline $\mathrm{CaCl}_{2}$ & Carl-Roth GmbH, Karlsruhe, Germany \\
\hline COC (20 $\mu \mathrm{m}$ thick $)$ & Topas 8007; TOPAS Advanced Polymers GmbH, \\
\hline COC $(240 \mu \mathrm{m}$ thick $)$ & $\begin{array}{l}\text { Frankfurt, Germany } \\
\text { msc foil 029; Microfluidic ChipShop GmbH, } \\
\text { Jena, Germany }\end{array}$ \\
\hline cover slips (thickness No. 1) & VWR, Radnor, PA, USA \\
\hline DTT & Carl-Roth GmbH, Karlsruhe, Germany \\
\hline Escherichia coli & $\begin{array}{l}\text { T61 cells \#3017, Zymo research, Irvine, Califor- } \\
\text { nia, USA }\end{array}$ \\
\hline EDTA & Carl-Roth GmbH, Karlsruhe, Germany \\
\hline EGTA & Carl-Roth GmbH, Karlsruhe, Germany \\
\hline fluorosilane & Sigma-Aldrich, Munich, Germany \\
\hline glass slides (76 mm x 26 mm) & Duran Group, Wertheim/Main, Germany \\
\hline glutaraldehyde & $\begin{array}{l}\text { Polysciences Europe GmbH, Hirschberg an der } \\
\text { Bergstrasse, Germany }\end{array}$ \\
\hline Gold colloids $10 \mathrm{~nm}$ & Sigma-Aldrich, Munich, Germany \\
\hline Gold colloids $15 \mathrm{~nm}$ & Sigma-Aldrich, Munich, Germany \\
\hline $\mathrm{HCl}$ & Carl-Roth GmbH, Karlsruhe, Germany \\
\hline HMDS & Sigma-Aldrich, Munich, Germany \\
\hline Hexammine-cobalt(III)chloride & Sigma-Aldrich, Munich, Germany \\
\hline Kapton foil ( $8 \mu \mathrm{m}$ thick) & $\begin{array}{l}\text { SPEX SamplePrep, Metuchen, USA, } 3511 \text { KAP- } \\
\text { TON }\end{array}$ \\
\hline $\mathrm{KCl}$ & Carl-Roth GmbH, Karlsruhe, Germany \\
\hline MAC & Sigma-Aldrich, Munich, Germany \\
\hline $\mathrm{MgCl}_{2}$ & Sigma-Aldrich, Munich, Germany \\
\hline Mica sheets (grad V5, 25 mm x $25 \mathrm{~mm}$ ) & 53-25; TED Pella, Inc., Redding, USA \\
\hline MOPS & Carl-Roth GmbH, Karlsruhe, Germany \\
\hline
\end{tabular}




\begin{tabular}{ll}
\hline Chemical & Company \\
\hline $\mathrm{mr}^{-D e v} 600$ (developer solution) & MicroChem, Newton, USA \\
$\mathrm{Na}_{2} \mathrm{HPO}_{4}$ & Carl-Roth GmbH, Karlsruhe, Germany \\
$\mathrm{NaCl}$ & Carl-Roth GmbH, Karlsruhe, Germany \\
$\mathrm{NaH}_{2} \mathrm{PO}_{4}$ & Carl-Roth GmbH, Karlsruhe, Germany \\
$\mathrm{NaOH}$ & Carl-Roth GmbH, Karlsruhe, Germany \\
$\mathrm{NOA} 81$ & Norland Optical Adhesives, Cranbury, NJ, USA \\
$\mathrm{PBS}$ & Invitrogen AG, Carlsbad, California, USA \\
$\mathrm{PDMS}$ & Dow Corning, Midland, USA \\
Spermine & Sigma-Aldrich, Munich, Germany \\
SU-8 2150 & MicroChem, Newton, USA \\
Sylgard 184 cross-linker & Dow Corning, Midland, USA \\
TRIS & Carl-Roth GmbH, Karlsruhe, Germany \\
urea & Carl-Roth GmbH, Karlsruhe, Germany \\
\hline
\end{tabular}




\section{References}

1. Block, J. et al. Nonlinear Loading-Rate-Dependent Force Response of Individual Vimentin Intermediate Filaments to Applied Strain. Phys. Rev. Lett. 118, 048101 (2017).

2. Winheim, S. et al. Deconstructing the Late Phase of Vimentin Assembly by Total Internal Reflection Fluorescence Microscopy (TIRFM). PLOS ONE 6 (ed Kreplak, L.) e19202 (2011).

3. Block, J. et al. Viscoelastic properties of vimentin originate from nonequilibrium conformational changes. Sci. Adv. 4, eaat1161 (2018).

4. Brennich, M. E. et al. Impact of ion valency on the assembly of vimentin studied by quantitative small angle X-ray scattering. Soft Matter 10, 2059-2068 (2014).

5. Huang, T. C., Toraya, H., Blanton, T. N. \& Wu, Y. X-ray powder diffraction analysis of silver behenate, a possible low-angle diffraction standard. J. Appl. Crystallogr. 26, 180-184 (1993).

6. Konarev, P. V., Volkov, V. V., Sokolova, A. V., Koch, M. H. J. \& Svergun, D. I. PRIMUS: a Windows PC-based system for small-angle scattering data analysis. J. Appl. Crystallogr. 36, 1277-1282 (2003).

7. Hémonnot, C. Y. J., Mauermann, M., Herrmann, H. \& Köster, S. Assembly of Simple Epithelial Keratin Filaments: Deciphering the Ion Dependence in Filament Organization. Biomacromolecules 16, 3313-3321 (2015).

8. Pedersen, J. S. Form factors of block copolymer micelles with spherical, ellipsoidal and cylindrical cores. J. Appl. Crystallogr. 33, 637-640 (2000).

9. Kornreich, M., Avinery, R., Malka-Gibor, E., Laser-Azogui, A. \& Beck, R. Order and disorder in intermediate filament proteins. FEBS Lett. 589, 2464-2476 (2015).

10. Strelkov, S. V., Schumacher, J., Burkhard, P., Aebi, U. \& Herrmann, H. Crystal Structure of the Human Lamin A Coil 2B Dimer: Implications for the Head-to-tail Association of Nuclear Lamins. J. Mol. Biol. 343, 1067-1080 (2004).

11. Mücke, N. et al. Molecular and Biophysical Characterization of Assembly-Starter Units of Human Vimentin. J. Mol. Biol. 340, 97-114 (2004).

12. Brennich, M. Cation induced self-assembly of intermediate filaments $\mathrm{PhD}$ thesis (Institute for X-Ray Physics, Georg-August-Universität Göttingen, 2012).

13. Eigel, M., Gruhlke, R., Marschall, M., Trunschke, P. \& Zander, E. ALEA - A Phython Framework for Spectral Methods and Low-Rank Approximations in Uncertainty Quantification https://bitbucket.org/aleadev/alea. 
14. Park, H. Y. et al. Achieving Uniform Mixing in a Microfluidic Device: Hydrodynamic Focusing Prior to Mixing. Anal. Chem. 78. PMID: 16808455, 4465-4473. eprint: http: //dx . doi . org/10.1021/ac060572n (2006).

15. Hofmann, I., Herrmann, H. \& Franke, W. W. Assembly and structure of calcium-induced thick vimentin filaments. Eur. J. Cell Biol. 56, 328-341 (1991).

16. Herrmann, H. et al. Structure and Assembly Properties of the Intermediate Filament Protein Vimentin: The Role of its Head, Rod and Tail Domains. J. Mol. Biol. 264, 933-953 (1996).

17. Weinhausen, B. \& Köster, S. Microfluidic devices for X-ray studies on hydrated cells. Lab Chip 13, 212-215 (2 2013).

18. Denz, M. et al. Cyclic olefin copolymer as an X-ray compatible material for microfluidic devices. Lab Chip 18, 171-178 (2018).

19. Schroer, C. G. et al. Hard x-ray nanoprobe based on refractive x-ray lenses. Appl. Phys. Lett. 87, 124103 (2005).

20. Henrich, B. et al. PILATUS: A single photon counting pixel detector for X-ray applications. Nucl. Instrum. Meth. A 607, 247-249. ISSN: 0168-9002 (2009).

21. Urbani, R. B. Dynamics in Microfluidics Measured by X-Ray Scattering Techniques PhD thesis (Philosophisch-Naturwissenschaftliche Fakultät, Universität Basel, 2015). 


\section{Chapter 4}

\section{Comparison of Different Buffers and their Effects on Intermediate Filaments}

\subsection{Importance of Buffers}

When performing experiments in vitro, they are mostly carried out in solutions. As the $\mathrm{pH}$ of the solvent can influence cell metabolism, chemical reactions or protein shapes, it is necessary that the $\mathrm{pH}$ of the solvent does not change too drastically during the experiments. The $\mathrm{pH}$ plays a crucial role in, e.g., the way vimentin subunits assemble to form mature filaments [1]. Furthermore, the $\mathrm{pH}$ can trigger the assembly of proteins [2]. To avoid uncontrolled changes in the $\mathrm{pH}$, it is of high importance to have an appropriate buffer system. The buffer stabilizes the $\mathrm{pH}$, however the buffer itself can influence biological systems as well. Therefore, the buffer needs to be chosen wisely according to the overall requirements during the experiments. In 1966, Good and his coworkers proposed a total of twelve so called Good buffers which should fulfill the criteria for good biological buffers [3, 4]. The original twelve pH-buffers introduced by Good et al. were completed by many more and nowadays a total of more than 30 Good buffers are available. According to Good et al., buffers should fulfill the following criteria: (I) The buffer should have a buffering range between $\mathrm{pH}$ 6-8, as many biological reactions take place in this regime. (II) It should have a maximal water solubility, meaning the $\mathrm{pH}$ should not change when diluting the buffer. (III) Furthermore, it should have a minimal lipid solubility, not to penetrate for example cell walls. (IV) The ionic strength of the system should not be influenced by the buffer. (V) The buffer should not be influenced by the temperature. (VI) There should be no influence of complexation of the buffer with cations and (VII) the buffer should be stable and have no enzyme inhibitory characteristics. (VIII) Additionally, the buffer should not absorb light above $240 \mathrm{~nm}$ as this could interfere with spectrometry. (IX) And lastly, the buffer should be easy to produce 
and inexpensive.

There is no buffer system available that can fulfill all criteria, Good et al. proposed, equally well. Even tough TRIS does not belong to the Good buffers it was proven to work for many biological experiments. However, one should keep in mind that TRIS buffers change the $\mathrm{pH}$ depending on the temperature. It has been shown, that TRIS buffer adjusted to a $\mathrm{pH}$ of 7.8 at room temperature has a $\mathrm{pH}$ of 7.4 at $37^{\circ} \mathrm{C}$ and a $\mathrm{pH}$ of 8.4 in the cold room [4]. Thus, TRIS is unsuitable in $\mathrm{pH}$ sensitive experiments when the temperature is changed. TRIS furthermore has a drastically reduced buffer capacity below a $\mathrm{pH}$ of 7.5 and is toxic for cells, as it can penetrate lipid membranes. Another frequently used and more physiological buffer system is PB. It is non-toxic, cheap and very easy to prepare. However, also this buffer system has disadvantages. The buffering capacity above $\mathrm{pH} 7.5$ is poor and the $\mathrm{pH}$ changes upon dilution. Furthermore, phosphate plays an active part in many biological processes e.g. the phosphorylation of vimentin [5] and thus the buffer can influence reactions.

Despite the general disadvantages both buffer systems work very well and are commonly used for IFs and in particular vimentin experiments [6-9], whereas the buffer for keratin experiments is mostly TRIS [10, 11]. Mücke et al. compared vimentin in TRIS and PB and showed that in both buffers ULFs as well as filaments are formed and no differences between the filaments assembled in one of the two buffer systems are observed [8]. However, it has been shown that both buffers interact with several ions. Larson et al. showed that PB can complex with polyvalent ions like $\mathrm{Ca}^{2+}[12]$. The interaction of divalent metal ions with TRIS was studied and complexing was shown with $\mathrm{Co}^{2+}$ and $\mathrm{Zn}^{2+}[13]$.

To study the effects of different ions on vimentin filaments and filament formation under comparable conditions, it is necessary that the buffer system does neither interact with one of the ions nor with the vimentin protein, e.g., initiate assembly of the vimentin protein.

There are many promising buffers to choose from, however we decided to take MOPS, which has a pKa of 7.2. As the $\mathrm{pH}$ during the experiment will be 7.5, the buffer used should have a pKa near 7.5, which MOPS fulfills. Several studies with MOPS and metal ions were performed during the last years leading to contradicting results on e.g. zinc and copper [14, 15]. Despite the contradicting results about complexation it is still concluded by Ferreira et al. that MOPS buffer is a non-complexing buffer [3]. However, interactions between buffer and vimentin protein still need to be investigated.

To investigate whether MOPS is also suitable for vimentin experiments, the behavior of vimentin protein is compared in low $(2 \mathrm{mM})$ and high $(20 \mathrm{mM})$ TRIS, PB and MOPS buffers. The lower concentration is chosen according to the standard vimentin assembly protocol [6-8]. The higher concentration is chosen to gain a better buffer capacity of the system. However, the probability that a higher buffer concentration influences the chemical balance of the system is high. 
The tetrameric state (meaning no assembly reagents added) as well as the assembled filament state of vimentin (addition of $100 \mathrm{mM} \mathrm{KCl}$ ) are investigated using AFM and SAXS. With AFM the overall filament structure can be observed, whereas SAXS yields information of the protein radius. While in $\mathrm{PB}$ the formation of filaments is initiated by the addition of $100 \mathrm{mM} \mathrm{KCl}$, the standard protocol for TRIS proposes a change of the $\mathrm{pH}$ from 8.4 to 7.5, an increase of the buffer concentration from $5 \mathrm{mM}$ to $25 \mathrm{mM}$ and the addition of $50 \mathrm{mM} \mathrm{NaCl}$ [8]. However, to make TRIS experiments more comparable to PB and MOPS, TRIS is kept at $2 \mathrm{mM}$ or $20 \mathrm{mM}$ concentration and the $\mathrm{pH}$ is not changed during the experiments shown here. Assembly is initiated by adding $100 \mathrm{mM} \mathrm{KCl}$, likewise to PB and MOPS experiments.

\subsection{Influence of the Buffers on Tetrameric Protein}

At first, the tetrameric state of vimentin is investigated in all three buffers at the low and high buffer concentration. The freshly dialyzed protein is prepared for AFM experiments and the re-
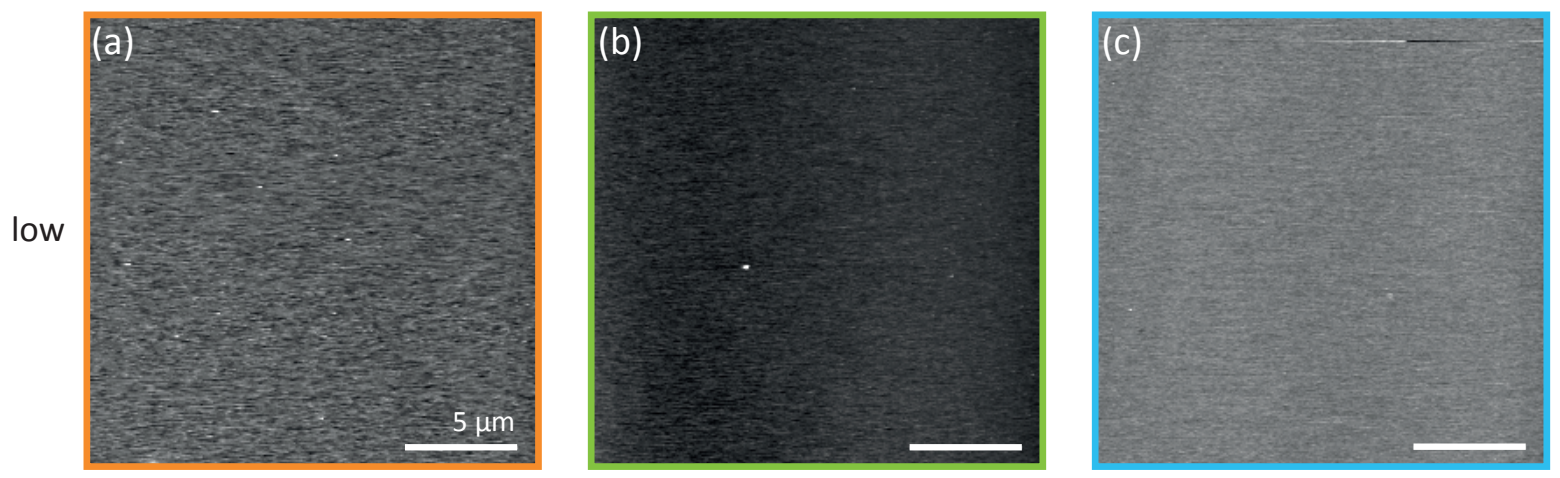

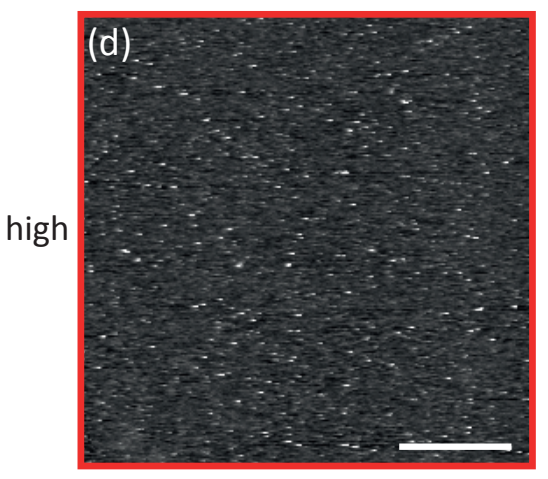

TRIS

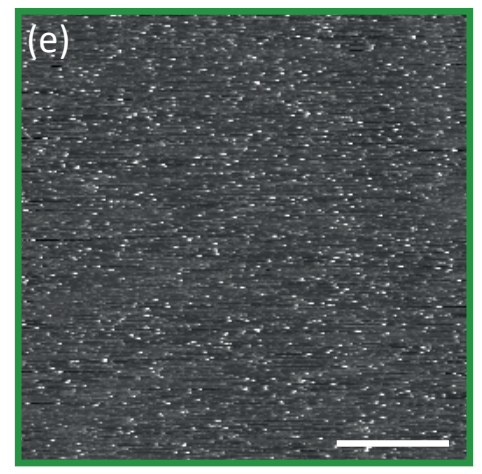

PB

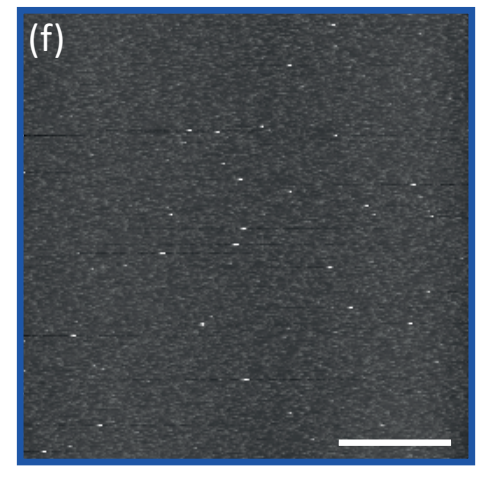

MOPS

Figure 4.1: Typical AFM images of tetrameric vimentin in different buffers: vimentin in 2 mM (a) TRIS, (b) PB and (c) MOPS buffer, each at $p H$ 7.5. Vimentin at the high buffer concentration of $20 \mathrm{mM}$ is shown in (d) TRIS, (e) PB, (f) MOPS buffer. Whereas nothing is visible at the low buffer concentration, small (roughly $200 \mathrm{~nm}$ long) vimentin aggregates are visible in the high buffer concentration. 
sults are presented in Fig. 4.1.

In Fig. 4.1 (a-c) vimentin protein in the three different buffers (TRIS, PB and MOPS) at low buffer concentration is shown. No aggregates are visible in any of the images, indicating that the structures in those solutions are smaller than what can be detected. In contrast, at high buffer concentration small structures, most likely aggregated or assembled vimentin protein, can be observed in all buffer systems. In the images, it seems that in TRIS and PB (Fig. 4.1d and e) more aggregates are visible than in MOPS buffer (Fig. 4.1f).

In addition to AFM experiments, SAXS measurements are performed three to six times per buffer and an example of all measurements at one condition are shown in Fig. 4.2.

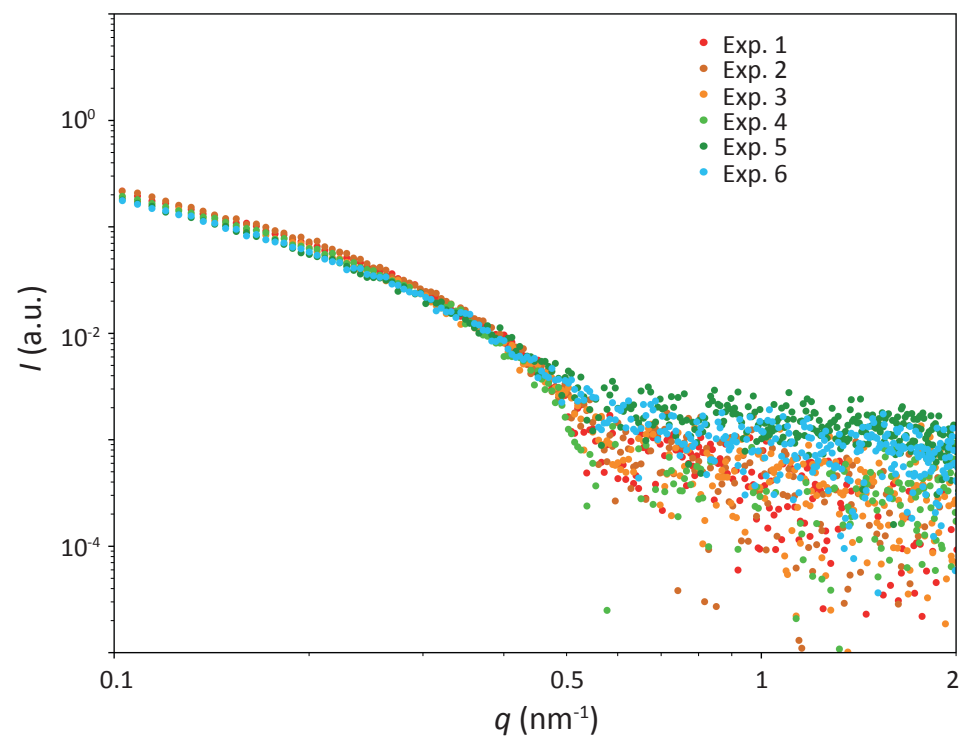

Figure 4.2: Scattering profiles of vimentin filaments assembled with $100 \mathrm{mM} \mathrm{KCl}$ in $2 \mathrm{mM} M O P S$ buffer. In total six different measurements are performed and the scattering profiles retrieved.

In Fig. 4.2 the scattering profiles of vimentin filaments assembled with $100 \mathrm{mM} \mathrm{KCl}$ in $2 \mathrm{mM}$ MOPS buffer are shown. For this experiment, six different experiments are conducted. All data are analyzed separately, however only one typical SAXS profile of vimentin protein in each buffer is shown in Fig. 4.3. An average is not calculated as the experiments are performed on different days and slight changes in the sample to detector distance are observed, which lead to a different $q$-range.

When looking at the scattering profiles of vimentin protein in the three buffers, it can be observed that there is no difference between the signals in the three buffers at low concentration, however the profiles of vimentin protein at high buffer concentrations are very different compared to the scattering profiles at low buffer concentration. The scattering curves of vimentin protein at low $q$-values are higher in intensity for the high buffer concentration than for vi- 


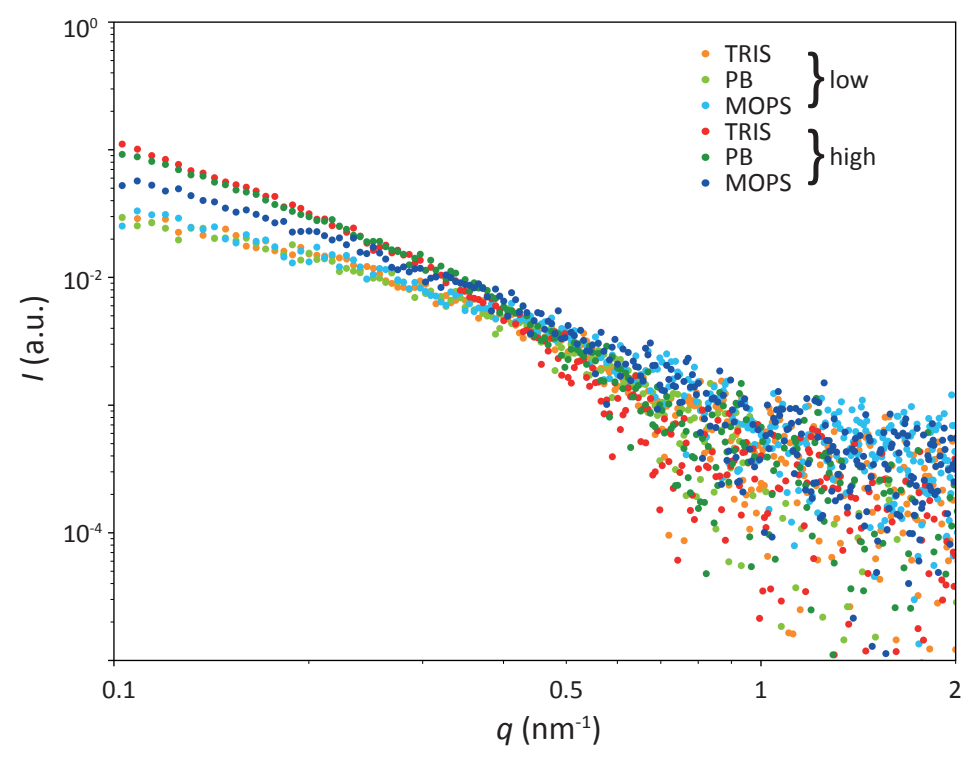

Figure 4.3: Scattering profiles of tetrameric vimentin at low $(2 \mathrm{mM})$ and high (20 $\mathrm{mM}$ ) buffer concentration. Three different buffers (TRIS, PB and MOPS) are tested. Scattering profiles of vimentin in the low buffer concentration are very similar, however in the high buffer concentration the scattering profiles look different.

mentin in the low buffer concentration. For the high TRIS and PB buffer concentration the intensity is even higher than for vimentin protein in the high MOPS concentration. The vimentin curves in the high buffer concentrations are steeper than those in the low buffer concentration, indicating that the protein radius increases. By performing a Guinier analysis of the data at small $q$, the radius of gyration for elongated objects $R_{c}$ as well as the $I(0)$ value can be extracted (Equation 2.27). Guinier analysis is performed on all data and the results for $R_{c}$ and $I(0)$ are shown in Fig. 4.4.

For the low buffer concentration, vimentin protein in all buffers has an average value of $R_{c} \approx$ $2.4 \mathrm{~nm}$, whereas for the high buffer concentration the radius of the vimentin protein varies from $R_{c} \approx 4.1 \mathrm{~nm}$ in MOPS to $R_{c} \approx 6.2 \mathrm{~nm}$ in TRIS buffer (Fig. $4.4 \mathrm{a}$ ). The $R_{c}$ values found for vimentin protein in low buffer concentration are in agreement with the literature [7]. The increase in the radius for vimentin at the higher buffer concentration is already seen in the AFM images, where under these conditions small structures, most likely vimentin filaments or aggregates were observed.

Fig. 4.4b shows the $I(0)$ values extracted from the Guinier fits. Having $I(0)$ on arbitrary unit scale, the values can not be used to compute the molecular weight, however the values can be compared with each other. The values for vimentin at the low buffer concentration are all very similar. On the contrary, the $I(0)$ values for vimentin protein in the high buffer concentration are different, following the same trend as the $R_{c}$ values (TRIS has the highest value and MOPS the lowest). 
(a)

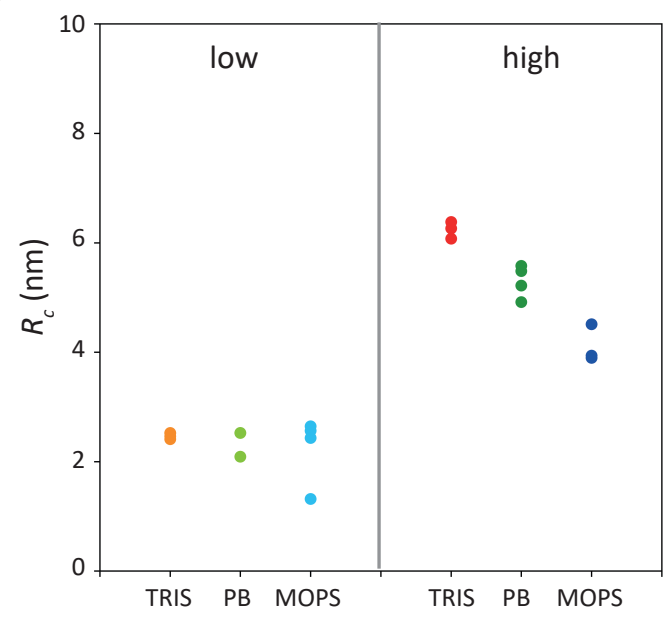

(b)

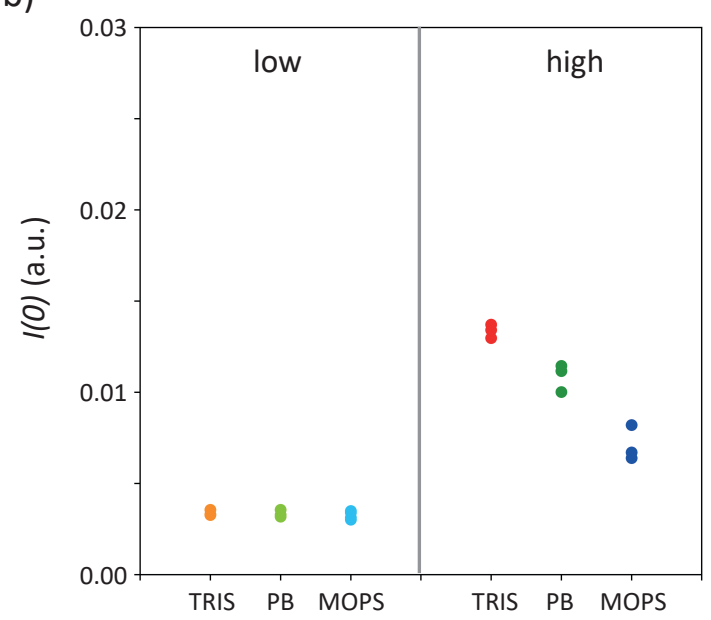

Figure 4.4: Guinier analysis of the scattering profiles of vimentin in the the three buffers (TRIS, PB and MOPS) at low $(2 \mathrm{mM})$ and high $(20 \mathrm{mM})$ concentration. (a) Radius of gyration $\left(R_{c}\right)$ for vimentin in the three different buffers at low and high buffer concentration. (b) $I(0)$ retrieved from the analysis for vimentin in the six different conditions. Similar $R_{c}$ and $I(0)$ values are found at the low buffer concentration. Different values are retrieved for the three buffers at the high buffer concentration.

\subsection{Influence of the Buffers on Filamental Protein}

All experiments performed with vimentin protein in the tetrameric state are repeated for vimentin after adding $100 \mathrm{mM} \mathrm{KCl}$. For vimentin in PB the addition of $100 \mathrm{mM} \mathrm{KCl}$ leads to the formation of mature filaments $[6,7,16]$. Typical AFM images of vimentin in all buffer conditions are shown in Fig. 4.5.

While there are long vimentin filaments at low buffer concentration and shorter filaments in the buffer with higher concentration, there seems to be no difference between the vimentin filaments in the different buffers at the same condition (Fig. 4.5). However, AFM experiments are only conducted to review whether filaments are observed at all or not. Therefore, these results should not be overinterpreted as the filament length was not quantified (due to the overlapping of the filaments), and the experiment was only performed twice. In addition, networks and aggregates of long vimentin filaments are likely to be washed away during sample preparation and therefore, not detected during the measurements. Thus, from the AFM experiments performed during this work, it cannot be conclusively said if the filament length varies between the low and the high buffer concentration. An analysis of the length distribution of the filaments could not be performed, due to the overlapping of the filaments.

Again, SAXS measurements are performed and the typical SAXS profiles of vimentin filaments in each buffer at the two different buffer concentrations are shown in Fig. 4.6.

The scattering profiles for all six conditions look very similar. The profiles start at similar inten- 

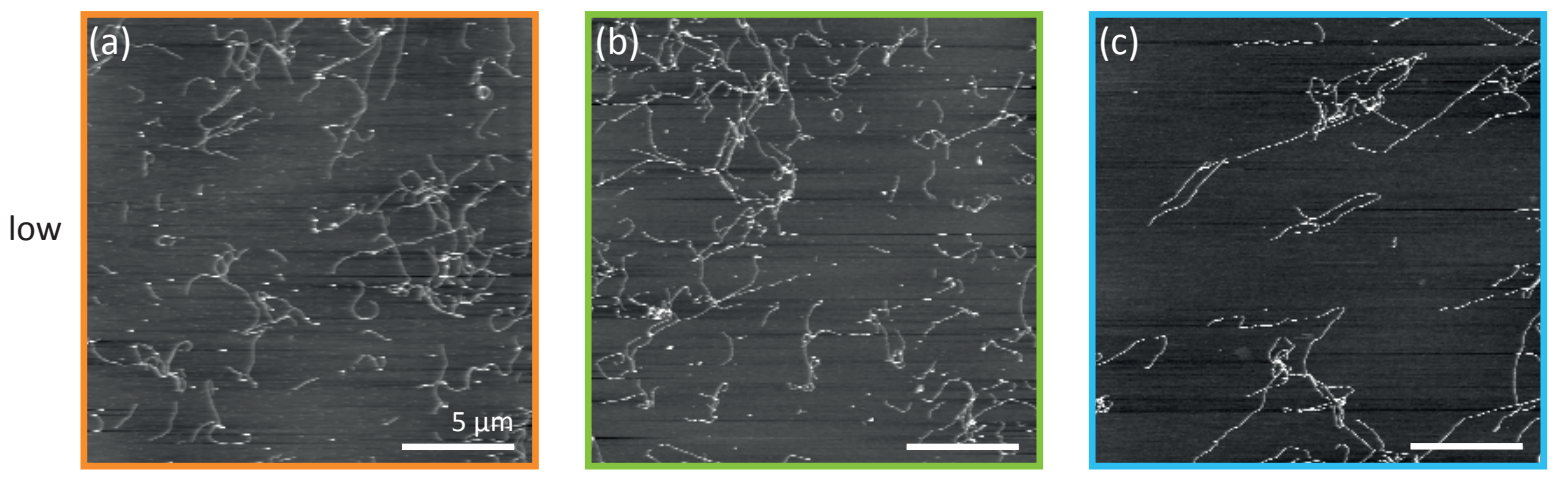

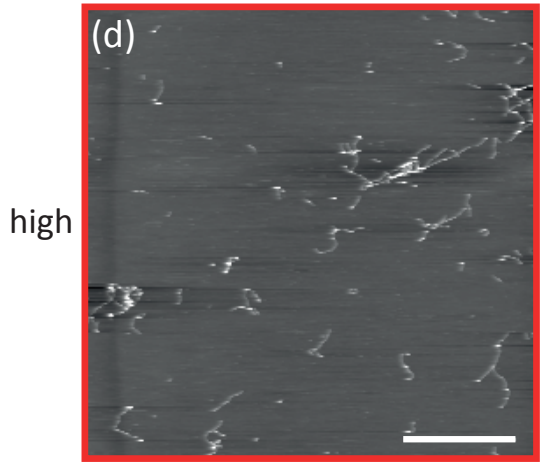

TRIS

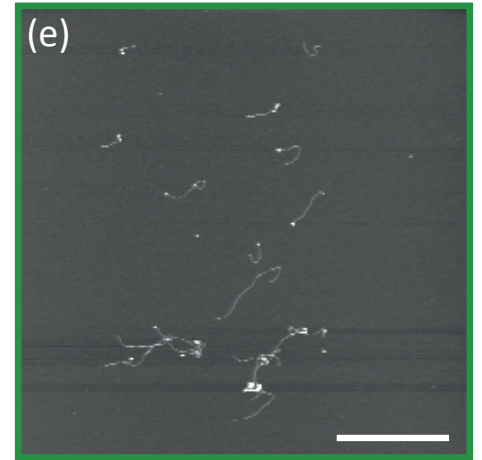

PB

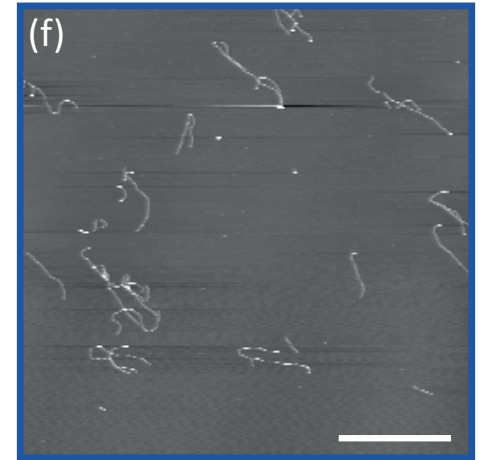

MOPS

Figure 4.5: AFM images of vimentin assembled to filaments in $100 \mathrm{mM} \mathrm{KCl}$ in different buffers: vimentin in $2 \mathrm{mM}$ (a) TRIS, (b) PB and (c) MOPS buffer, each at pH 7.5. Vimentin at the high buffer concentration of $20 \mathrm{mM}$ is shown in (d) TRIS, (e) PB, ( $f$ ) MOPS buffer. At the low buffer concentration, vimentin forms elongated filaments and no difference between the different buffers can be seen. At the high buffer concentration, filaments form as well. However, the imaged filaments are shorter and fewer.

sity values at low $q$. However, the profiles of vimentin filaments at high buffer concentration are steeper than the scattering profiles of vimentin filaments at low buffer concentration. Especially the vimentin filament curves in high TRIS and PB are steeper than the others. Again, the Guinier analysis is performed and the results are displayed in Fig. 4.7.

When looking at the $R_{c}$ values (Fig. $4.7 \mathrm{a}$ ) no difference between vimentin assembled in the three buffers at low buffer concentration can be observed. The filaments have a radius of gyration of $R_{c} \approx 5.6 \mathrm{~nm}$, which is in agreement with the literature [7]. On the contrary, the $R_{c}$ values for vimentin filaments in the high buffer concentration are larger and range from $R_{c} \approx 6.3 \mathrm{~nm}$ for vimentin in MOPS buffer to $R_{c} \approx 7.4 \mathrm{~nm}$ for filaments in TRIS buffer. Looking at the $I(0)$ values retrieved from the Guinier analysis (Fig. 4.7), the values for vimentin filaments in low buffer concentration are all very similar (Fig. 4.7b). For the high buffer concentration the retrieved $I(0)$ values are very similar to each other and similar to the values retrieved for vimentin filaments in the low buffer concentration. 


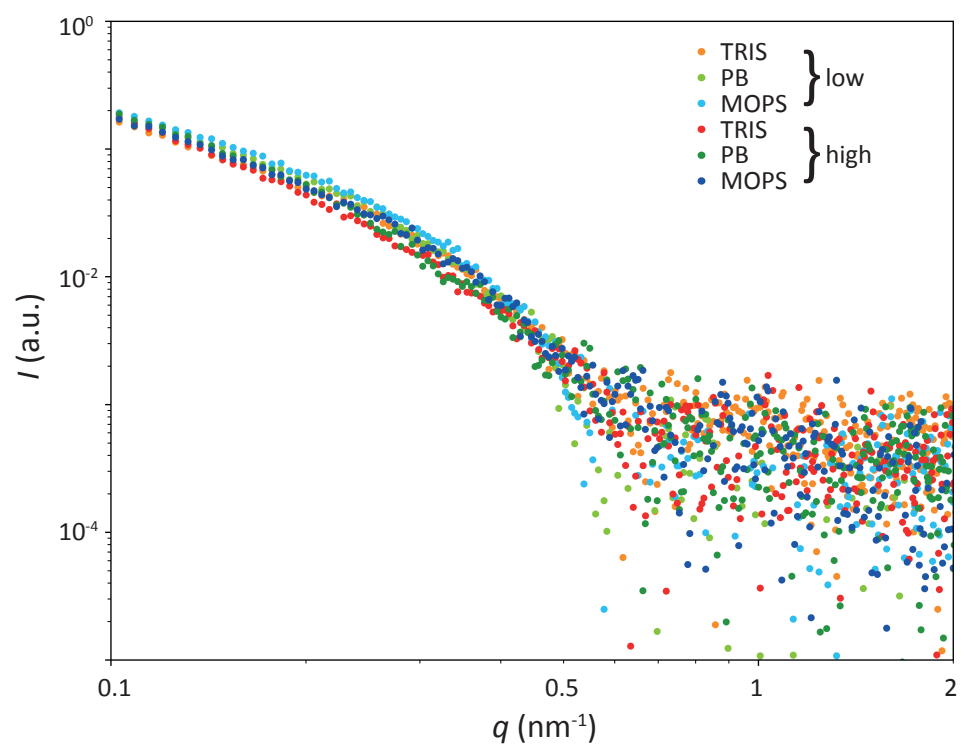

Figure 4.6: Scattering profiles of vimentin filaments at low (2 $\mathrm{mM})$ and high (20 $\mathrm{mM})$ buffer concentrations. Three different buffers (TRIS, PB and MOPS) are measured. All scattering profiles look similar. However, the curves for vimentin filaments at the higher buffer concentrations are slightly steeper.

(a)

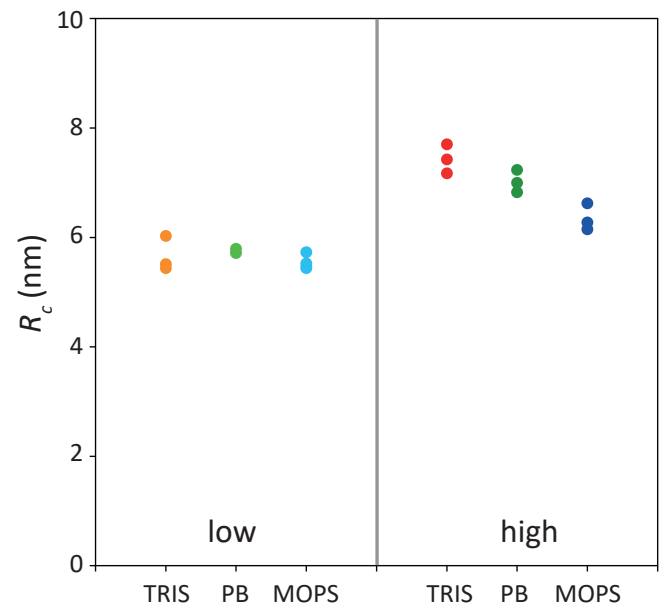

(b)

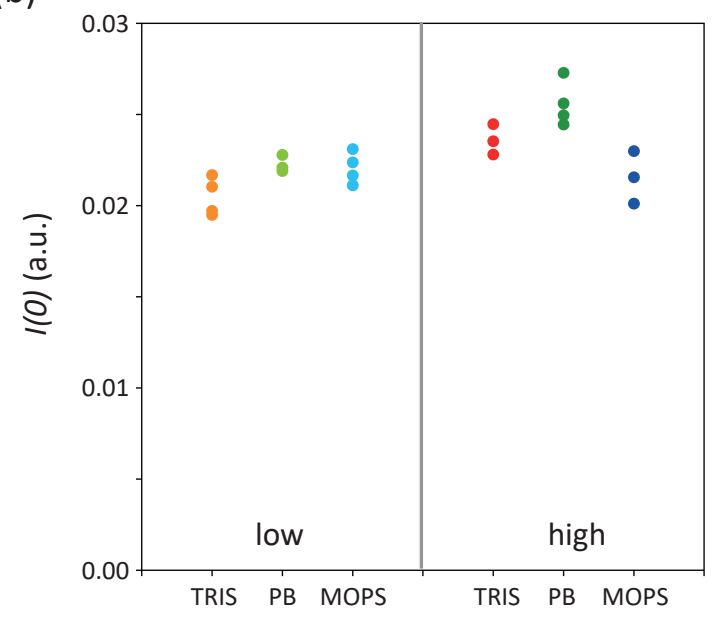

Figure 4.7: Guinier analysis of the scattering profiles of vimentin filaments in the low (2 mM) and high (20 mM) concentrations of the three buffers (TRIS, PB and MOPS). (a) Radius of gyration $\left(R_{c}\right)$ for vimentin at low and high buffer concentration in TRIS, PB and MOPS buffer. (b) I(0) values retrieved from the analysis for vimentin filaments. Similar $R_{c}$ and $I(0)$ values are found at the low buffer concentration. The $R_{c}$ values are slightly larger in the high buffer concentration, however the $I(0)$ values are very similar in all six conditions.

\subsection{Summary and Discussion}

To rule out an influence of the buffer used for assembly experiments, three different buffers (TRIS, PB and MOPS) are investigated at a low (2 mM) and high ( $20 \mathrm{mM})$ concentration. $\mathrm{PB}$ and 
TRIS buffer are commonly used for vimentin experiments, however both buffers interact with several ions e.g. $\mathrm{Ca}^{2+}$ or $\mathrm{Zn}^{2+}[12,13]$. To investigate the influence of ions on the assembly of vimentin, it is of high importance to have a buffer which does not interact with the ions. Summarizing the AFM and SAXS experiments, it can be concluded that there is no obvious difference between the tetramers and filaments measured in the three buffers at a low buffer concentration. The AFM images for the tetrameric state (Fig. 4.1a-c) are all similar showing no aggregated vimentin structures. Also, the filaments are similarly assembled in the three buffers at low concentration (Fig. 4.5a-c). From the AFM experiments no difference in the behavior of vimentin in the buffers can be detected. The performed SAXS experiments indicate the same. Scattering curves for tetrameric vimentin (Fig. 4.3) as well for the filamental state (Fig. 4.6) show no difference for different buffers at low buffer concentration. Guinier analysis of vimentin in the low buffer concentration results in similar $R_{c}$ and $I(0)$ values for all three buffers. The tetrameric state yields a value of roughly $R_{c} \approx 2.4 \mathrm{~nm}$ and the filamental state a value of $R_{c} \approx 5.6 \mathrm{~nm}$ which is in agreement with the literature $[7,17,18]$. The $I(0)$ values can not be related to the molecular weight as the data are not on absolute scale, however all measurements are taken at the same setup and therefore, should be multiplied by the same CF. Thus, even if the CF is not known, similar $I(0)$ values would result in similar molecular weights on absolute scale. Therefore, it can be concluded that if the $I(0)$ values are similar they would result in similar molecular weights. The $I(0)$ values are higher for the assembled vimentin which is plausible as the molecular weight should increase for the assembled vimentin. Taking Equation 2.24, it can be observed that for calculating the molecular weight from the $I(0)$ values, the molecular weight depends on the concentration $c$, the specific volume of the particle $v$ and the scattering contrast. The scattering contrast should be the same for tetrameric and filamental vimentin. SAXS measurements are all performed at the same concentration $(1 \mathrm{mg} / \mathrm{mL})$, thus it is the same for tetramers and filaments. The particles specific volume only changes very little or not at all between the tetrameric and filamental state. Thus, the molecular weight is nearly proportional to the $I(0)$ values. On average eight tetramers assemble laterally to form an ULF [7, 19]. This would indicate that the molecular weight increases by roughly a factor of eight as well. Looking at the data, the ratio of the filamental $I(0)$ to the tetramer $I(0)$ is not eight but slightly smaller (approx. 7). This could have several reasons. One reason could be that the particles specific volume changes a bit during assembly e.g by reduction of the volume because of compaction. Then the ratio between both $I(0)$ values should get smaller than eight. Another reason could be, that the tetrameric state already includes octamers or 16-mers, which would increase the measured $I(0)$ value. At a $\mathrm{pH}$ of 8.4, the protein solution should only consist of tetramers, whereas at a $\mathrm{pH}$ of 7.5 eventually already octamers could be present. During an experiment vimentin protein in $2 \mathrm{mM} \mathrm{PB}$ at a $\mathrm{pH}$ of 8.4 and 7.5 are measured (Fig. 4.8a). 


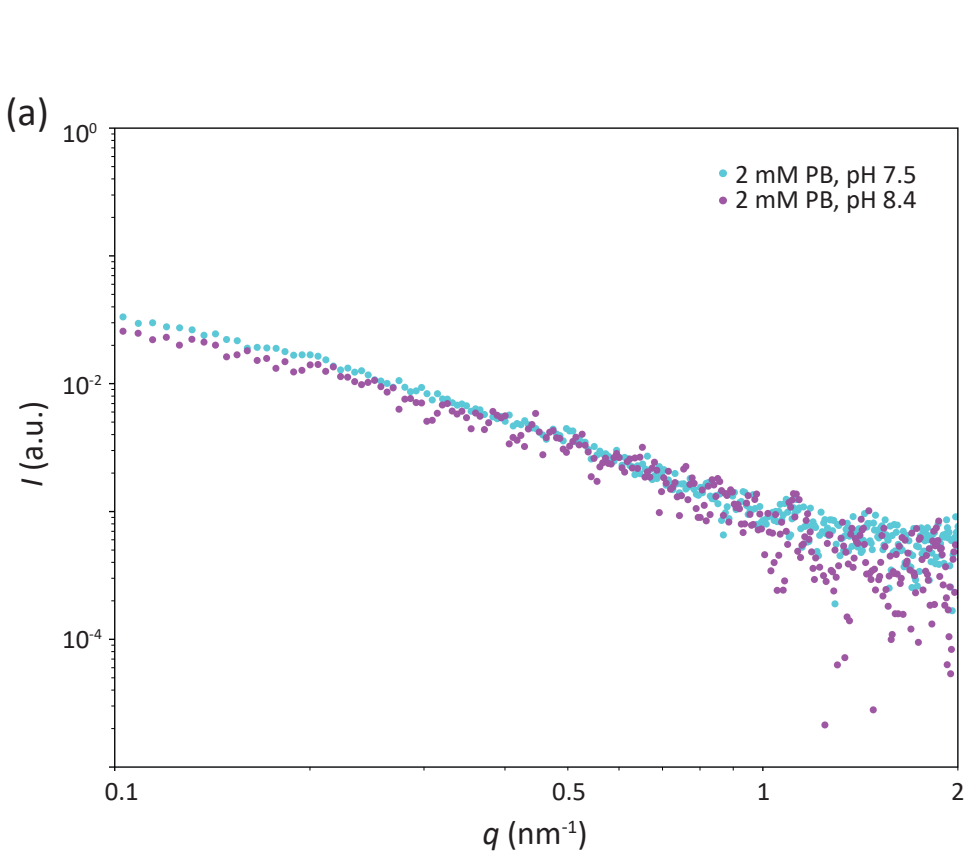

(b)

(c)
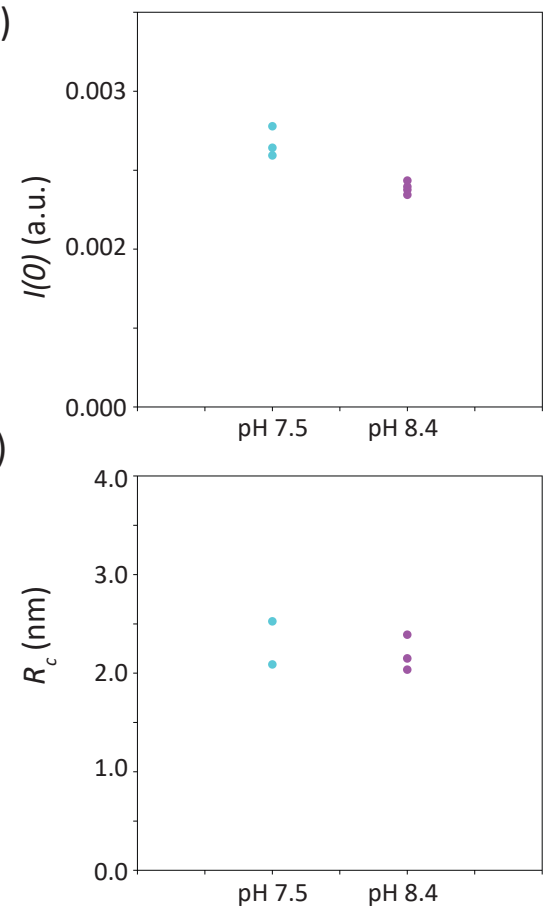

Figure 4.8: Comparison of vimentin tetramers in $P B$ at $p H$ 7.5 and 8.4. (a) Scattering profiles (b) I(0) values and (c) $R_{c}$ values of vimentin tetramers in $P B$ at two different $p H$ values. Only very small changes between the measurements can be observed.

We can observe that the $I(0)$ value is lower than the value retrieved from measurements at $\mathrm{pH}$ 7.5 (Fig.4.8b), whereas the $R_{c}$ values are similar (Fig. 4.8c). The smaller values at higher $\mathrm{pH}$ indicate that there is a small fraction of octamers or higher order structures at $\mathrm{pH}$ 7.5. A third plausible reason for the difference in the $I(0)$ values is the possible inhomogeneity of the filaments. Variations in width and in the number of subunits within one filament have been reported [1, 20], especially if assembly is initiated with the kick-start method, where the filaments become even more inhomogeneous than with slow assembly through dialysis [21]. For all experiments shown, the kick-start method is used to initiate assembly, and thus a larger inhomogeneity in the filaments is expected.

At the high buffer concentrations already in the tetrameric state larger structures are observed with AFM and SAXS, indicating aggregates or assembled filaments already due to the higher buffer concentration. This is also reflected in the $R_{c}$ and $I(0)$ values (Fig. 4.4). Both the radii of gyration of the cross-section and the $I(0)$ values are two to three times larger compared to vimentin at low buffer concentration. Both, AFM and SAXS measurements, show that a higher buffer concentration has an effect on the vimentin filament formation or aggregation. Regarding the filamentous state of vimentin, the $R_{c}$ values are slightly larger than those found in lit- 
erature. However, the $I(0)$ values are similar for vimentin filaments in the high and low buffer concentrations. Having similar $I(0)$ values indicates that the filaments at low and high buffer concentration have a similar molecular weight. The $R_{c}$ values for vimentin in the high buffer concentration are however slightly larger, which leads to the assumption that the number of vimentin subunits per cross-section is similar for the low and high buffer concentration. However, the larger radii of gyration suggest a looser packing of the subunits or a less homogeneous filament at the high buffer concentration.

In addition to AFM and SAXS experiments, Anna Schepers (unpublished data, University Göttingen) used an optical trap to perform stretching experiments on vimentin C328A with $4 \%$ ATTO647N labeled vimentin. Vimentin C328A is assembled in $2 \mathrm{mM}$ PB with $100 \mathrm{mM}$ KCl. For experiments, the filaments are bound covalently to the beads in the trap. A more detailed description on how stretching experiments using an optical trap with vimentin filaments is performed are found in $[6,22]$. In the experiments shown here, filaments are incubated $30 \mathrm{~s}$ in the desired buffer and then stretched with a speed of $0.25 \mu \mathrm{m} / \mathrm{s}$. Filaments in $2 \mathrm{mM}$ PB with $100 \mathrm{mM}$ $\mathrm{KCl}$ or $2 \mathrm{mM}$ MOPS with $100 \mathrm{mM} \mathrm{KCl}$ are investigated. Additionally, filaments are incubated in $25 \mathrm{mM}$ TRIS buffer with $80 \mathrm{mM} \mathrm{NaCl}$. The results are shown in Fig. 4.9.

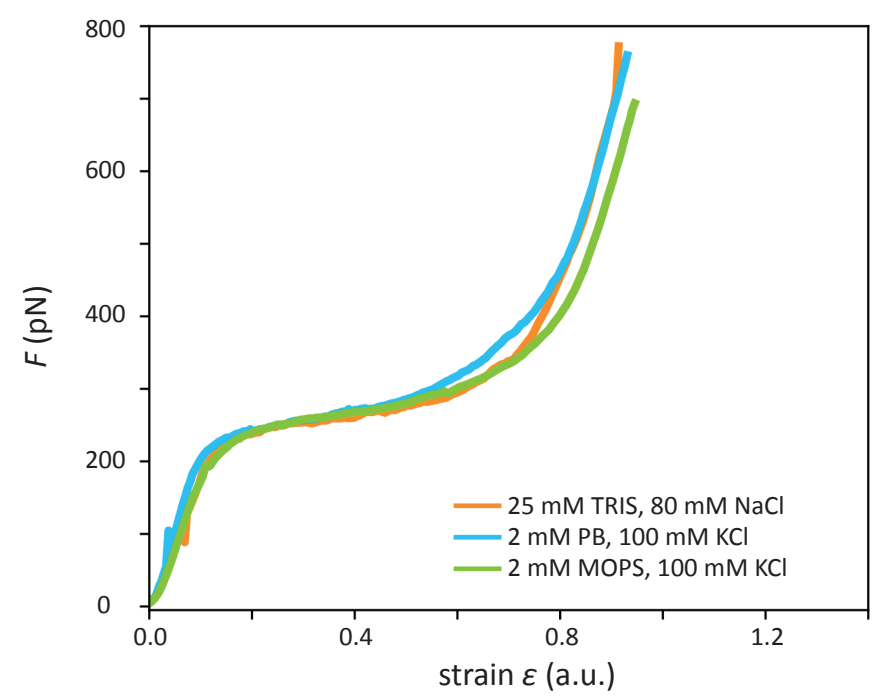

Figure 4.9: Force strain curves for vimentin filaments. Vimentin filaments are assembled in $2 \mathrm{mM}$ PB buffer and prior to measurement incubated for $30 \mathrm{~s}$ in different buffers (TRIS, PB and MOPS). The force strain curves for 2 mM PB, 2 mM MOPS and 25 mM TRIS buffer are all very similar. Data and image courtesy by Anna Schepers.

A full description on how to interpret vimentin force-strain curves is mentioned in [6, 22]. In general, the curve can be divided in three regions. The initial slope, the plateau and the stiffening region. Looking at the data, no difference in the force-strain curves of filaments in the 
buffers can be detected. For the three tested buffers, the initial slope is the same, the plateau regions start at the same strain and the maximum force and strain are similar as well. Thus, it can be concluded that all buffer conditions seem to have no effect, or the same effect on the filament.

Taking together all results, it can be concluded that there is no difference between vimentin filaments in the three tested buffers at low buffer concentration. For the high buffer concentration, it seems that all buffers have an effect on vimentin protein, however this effect is less pronounced for the filaments than for the tetrameric state. Therefore, high buffer concentrations should not be used for vimentin experiments, especially when the tetrameric state is investigated. Furthermore, one should keep in mind that AFM and SAXS experiments are static experiments and only the tetrameric state or the fully assembled vimentin is investigated. It might be that the assembly process itself varies between the buffers. To investigate if the assembly process is similar as well, time-resolved studies have to be performed. This could be done using e.g SAXS combined with microfluidics. Time-resolved SAXS experiments are described in Chapter 6. Those experiments could not be performed in the scope of this thesis, as they have to be performed at a synchrotron.

Even if the literature states that neither PB nor TRIS is a Good buffer [3, 4], they are sufficient for vimentin assembly and lead to no artifacts. Already Mücke et al. showed that there is no difference in the formation of filaments between assembly of vimentin in $22.5 \mathrm{mM}$ TRIS with $50 \mathrm{mM}$ $\mathrm{NaCl}$ (pH 7.5) and PB with additional $100 \mathrm{mM} \mathrm{KCl} \mathrm{(pH} \mathrm{7.5)} \mathrm{[8].} \mathrm{Here,} \mathrm{we} \mathrm{saw} \mathrm{similar} \mathrm{results} \mathrm{and}$ could furthermore show, that also MOPS is a suitable buffer when investigating vimentin assembly. All in all it can therefore be concluded, that MOPS as well as TRIS and PB can be used in low concentrations to investigate vimentin. 


\section{References}

1. Herrmann, H., Häner, M., Brettel, M., Ku, N.-O. \& Aebi, U. Characterization of distinct early assembly units of different intermediate filament proteins. J. Mol. Biol. 286, 1403-1420 (1999).

2. Wallace, J. A. \& Shen, J. K. Unraveling a Trap-and-Trigger Mechanism in the pH-Sensitive Self-Assembly of Spider Silk Proteins. J. Phys. Chem. Lett. 3, 658-662 (2012).

3. Ferreira, C. M. H., Pinto, I. S. S., Soares, E. V. \& Soares, H. M. V. M. (Un)suitability of the use of $\mathrm{pH}$ buffers in biological, biochemical and environmental studies and their interaction with metal ions - a review. RSC Advances 5, 30989-31003 (2015).

4. Good, N. E. et al. Hydrogen Ion Buffers for Biological Research. Biochemistry 5, 467-477 (1966).

5. Eriksson, J. E. et al. Specific in vivo phosphorylation sites determine the assembly dynamics of vimentin intermediate filaments. J. Cell Sci. 117, 919-932 (2004).

6. Block, J. et al. Nonlinear Loading-Rate-Dependent Force Response of Individual Vimentin Intermediate Filaments to Applied Strain. Phys. Rev. Lett. 118, 048101 (2017).

7. Brennich, M. E. et al. Impact of ion valency on the assembly of vimentin studied by quantitative small angle X-ray scattering. Soft Matter 10, 2059-2068 (2014).

8. Mücke, N. et al. Molecular and Biophysical Characterization of Assembly-Starter Units of Human Vimentin. J. Mol. Biol. 340, 97-114 (2004).

9. Premchandar, A. et al. Structural Dynamics of the Vimentin Coiled-coil Contact Regions Involved in Filament Assembly as Revealed by Hydrogen-Deuterium Exchange. J. Biol. Chem. 291, 24931-24950 (2016).

10. Premchandar, A. et al. Analysis of distinct molecular assembly complexes of keratin K8 and K18 by hydrogen-deuterium exchange. J. Struct. Biol. 192, 426-440 (2015).

11. Hémonnot, C. Y. J., Mauermann, M., Herrmann, H. \& Köster, S. Assembly of Simple Epithelial Keratin Filaments: Deciphering the Ion Dependence in Filament Organization. Biomacromolecules 16, 3313-3321 (2015).

12. Larson, M. C., Luthi, M. R., Hogg, N. \& Hillery, C. A. Calcium-phosphate microprecipitates mimic microparticles when examined with flow cytometry. Cytometry Part A 83A, 242-250 (2012).

13. Hanlon, D. P., Watt, D. S. \& Westhead, E. W. The interaction of divalent metal ions with tris buffer in dilute solution. Anal. Biochem. 16, 225-233 (1966). 
14. Mash, H. E., Chin, Y.-P., Sigg, L., Hari, R. \& Xue, H. Complexation of Copper by Zwitterionic Aminosulfonic (Good) Buffers. Anal. Chem. 75, 671-677 (2003).

15. Anwar, Z. M. \& Azab, H. A. Ternary Complexes in Solution. Comparison of the Coordination Tendency of Some Biologically Important Zwitterionic Buffers toward the Binary Complexes of Some Transition Metal Ions and Some Amino Acids. J. Chem. Eng. Data 44, 11511157 (1999).

16. Dammann, C., Nöding, B. \& Köster, S. Vimentin networks at tunable ion-concentration in microfluidic drops. Biomicrofluidics 6, 022009 (2012).

17. Kreplak, L. \& Fudge, D. Biomechanical properties of intermediate filaments: from tissues to single filaments and back. Bioessays 29, 26-35 (2006).

18. Steinert, P. M., Idler, W. W. \& Zimmerman, S. B. Self-assembly of bovine epidermal keratin filaments in vitro. J. Mol. Biol. 108, 547-567 (1976).

19. Dammann, C., Herrmann, H. \& Köster, S. Competitive Counterion Binding Regulates the Aggregation Onset of Vimentin Intermediate Filaments. Isr. J. Chem. 56, 614-621 (2015).

20. Block, J., Schroeder, V., Pawelzyk, P., Willenbacher, N. \& Köster, S. Physical properties of cytoplasmic intermediate filaments. Biochim. Biophys. Acta, Mol. Cell Res. 1853, 30533064 (2015).

21. Köster, S., Weitz, D. A., Goldman, R. D., Aebi, U. \& Herrmann, H. Intermediate filament mechanics in vitro and in the cell: from coiled coils to filaments, fibers and networks. Curr. Opin. Cell Biol. 32, 82-91 (2015).

22. Block, J. et al. Viscoelastic properties of vimentin originate from nonequilibrium conformational changes. Sci. Adv. 4, eaat1161 (2018). 


\section{Chapter 5}

\section{The Influence of Ions on the Assembly of Intermediate Filaments}

\subsection{The Role of Ions for Intermediate Filaments}

Ions play an integral role in many processes within the cell. The cell itself contains various ions such as $\mathrm{K}^{+}$and $\mathrm{Na}^{+}$, which are present at concentrations around $150 \mathrm{mM}$ [1]. Next to monovalent ions like $\mathrm{K}^{+}$and $\mathrm{Na}^{+}$there are also ions of higher valency in the cell, where the most abundant multivalent ions are $\mathrm{Ca}^{2+}, \mathrm{Mg}^{2+}, \mathrm{Zn}^{2+}$ and $\mathrm{Sp}^{4+}$ (spermine) [1]. Various ions in the cell and also in the cytoplasm will have an affect on cytoskeletal filaments as well. Even though the assembly of IFs in cells is not only mediated by ions, they play a part in the assembly. In vitro, the assembly of vimentin can be triggered by adding monovalent ions to the solution [2-4]. The resulting IFs are polyelectrolytes with an overall negative net charge [1,5]. Vimentin filaments with a radius of $5 \mathrm{~nm}$ and 32 subunits, for example, exhibit a net charge of -19 e per monomer at a $\mathrm{pH}$ of 7.5 , which equals a line charge of $-14 \mathrm{e} / \mathrm{nm}$ and a surface charge of $-71 \mathrm{mC} / \mathrm{m}^{2}[5$, 6]. The charge is not evenly distributed over the whole monomer, but exhibiting a positive net charged head domain and a tail domain with a negative net charge. In Figure 5.1 the amino acid sequence of a vimentin monomer is shown with the negatively (green) and positively (red) charged amino acids highlighted.

When investigating the influence of ions on IFs, two different aspects can be examined. Firstly, by adding ions of high valency to already formed filaments, the interaction of mature filaments with the ions can be studied. Secondly, the influence of ions with different valencies on the tetramer can be explored, by which the influence of the ions on the assembly is examined. The influence of monovalent ions on the assembly of IFs has been widely studied but the interaction of IFs with multivalent ions is not completely understood. Studies revealed that the addition of 


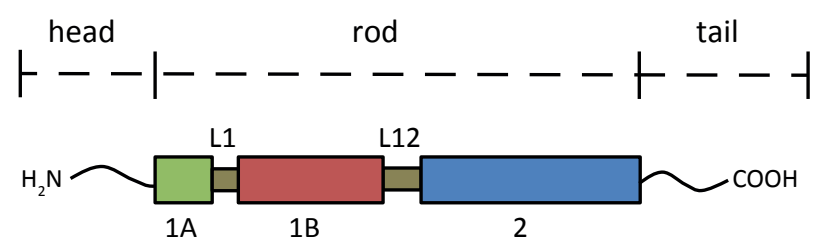

MATRSVSSSS YRRMFGGPGT ASRPSSSRSY VTTSTRTYSL GSALRPSTSR SLYASSPGGV YATRSSAVRL RSSVPGVRLL QDSVDFSLAD AINTEFKNTR TNEKVELQEL NDRFANYIDK VRFLEQQNKII LLAELEQLKG QGKSRLGDLY EEEMRELRRQ VDQLTNDKAR VEVERDNLAE DIMRLREKLQ EEMLQREEAE NTLQSFRODV DNASLARLDL ERKVESLQEE IAFLKKLHEE EIQELQAQIQ EQHVQIDVDV SKPDLTAALR DVRQQYESVA AKKNLQEAEEW YKSKKFADLSE AANRNNDALR QAKOESTEYR RQVQSLTCEV DALKGTNESL ERQMREMEEN FAVEAANYQD TIGRLQDEIQ NMKEEMARHL REYQDLLNVK MALDIEIATY RKLLEGEESR ISLPLPNFSS LNLRETNLDS LPLVDTHSKR TLLIKTVETR DGQVINETSQ HHDDLE

charged + charged -

Figure 5.1: Scheme of a vimentin monomer and the corresponding amino acids sequence of vimentin. Positively charged amino acids are highlighted in red and negatively charged amino acids in green. Above the amino acid sequence, a schematic representation of the vimentin monomer is shown. The three parts of the rod domain (colored in green, red and blue) are highlighted in the amino acid sequence, for a better overview.

divalent ions to mature filaments caused network formation as well as bundling and aggregation [1, 7-9], Damman et al. showed that with increasing divalent ion concentration, freely fluctuating filaments collapse to dense aggregated networks $[4,5]$. Furthermore, it was shown that for neurofilaments, no significant difference between the resulting networks formed by different divalent ions $\left(\mathrm{Ca}^{2+}, \mathrm{Mg}^{2+}, \mathrm{Zn}^{2+}\right)$ is observed [7]. The mentioned studies all investigated the influence of multivalent ions on filaments. When examining the influence of ions on the assembly, thus adding the ions to the tetramers, several studies have shown that there are differences between IFs in the presence of different ions. It has been observed that desmin assembled with $\mathrm{Ca}^{2+}$ has a larger diameter than filaments assembled with $\mathrm{Na}^{+}[10]$. This has not only been reported for desmin but also for vimentin using $\mathrm{Mg}^{2+}$ ions $[2,11,12]$. Stromer et al. concluded that not only the ionic strength of the buffer is changed when different ions are added but also the interaction of the ion with the filament changes. They showed that at similar ionic strength, divalent ions like $\mathrm{Mg}^{2+}$ are a stronger promoter for desmin assembly than monovalent ions [13]. Hofmann et al. showed that filaments assembled in the presence of $\mathrm{Mg}^{2+}$ are similar to vimentin assembled with monovalent ions, however vimentin filaments assembled by $\mathrm{Ca}^{2+}$ are 
more inhomogeneous and thicker [10]. Wu et al. investigated the influence of $\mathrm{Ca}^{2+}$ and $\mathrm{Zn}^{2+}$ on the assembly of vimentin protein. They reported, that vimentin in the presence of $\mathrm{Zn}^{2+}$ ions bundles at a lower ion concentration than when $\mathrm{Ca}^{2+}$ is added to vimentin proteins [14]. Brennich et al. showed in SAXS experiments that vimentin assembled with $\mathrm{MgCl}_{2}$ leads to thicker and less ordered structures compared to vimentin assembled with $\mathrm{KCl}$ [2]. Those findings already showed that adding different multivalent ions to tetrameric IFs does not lead to the same filament diameter, but that every ion needs to be investigated individually.

It has already been shown that there is not only a difference in the assembled filament when adding monovalent or divalent ions, but also between different divalent ions [10, 14]. Therefore, we investigate the influence of different mono- and divalent ions, as well as the influence of a trivalent ion and a tetravalent molecule on the assembly of vimentin protein in a systematic way. The influence of $\mathrm{Na}^{+}, \mathrm{K}^{+}, \mathrm{Mg}^{2+}, \mathrm{Ca}^{2+}, \mathrm{Co}^{3+}$ and $\mathrm{Sp}^{4+}$ at various concentrations will be tested. All tested salts have $\mathrm{Cl}^{-}$as the counter ion, except $\mathrm{Sp}^{4+}$. It has been reported that $\mathrm{Cl}^{-}$ does not have a dominant influence on the filaments if it is present with $\mathrm{K}^{+} \mathrm{or}^{2+} \mathrm{gg}^{2+}$ [15]. As a buffer system $2 \mathrm{mM}$ MOPS has been chosen, as MOPS does not interact with the ions under investigation (see Chapter 4). To study the influence of the different ions, SAXS is used to probe the lateral assembly of the protein, whereas fluorescence microscopy is used to investigate if vimentin protein forms filaments or networks.

\subsection{Vimentin Filaments Assembled in the Presence of Monova- lent Ions}

The influence of monovalent ions on the assembly of vimentin protein from tetramers to filaments in the presence of $\mathrm{KCl}$ and $\mathrm{NaCl}$ is investigated. $\mathrm{K}^{+}$and $\mathrm{Na}^{+}$are chosen as they are the most abundant monovalent ions in the cell [1]. SAXS experiments are performed with both monovalent ions at eight different concentrations ranging from $10 \mathrm{mM}$ to $150 \mathrm{mM}$. This range is chosen, as both ions reach concentrations up to $150 \mathrm{mM}$ in the cell [1]. The scattering profiles for vimentin assembled in the presence of $\mathrm{KCl}$ and $\mathrm{NaCl}$ are shown in Fig. 5.2.

In Fig. 5.2a, the scattering profiles of vimentin assembled with $\mathrm{KCl}$ are shown, where the black curve refers to the tetrameric state, before ions are added. The scattering profile for tetrameric vimentin protein is measured for $24 \mathrm{~h}$ and therefore, the curve is less noisy than the other scattering curves, which are only recorded for $4 \mathrm{~h}$. Vimentin tetramers are measured for $24 \mathrm{~h}$, as this scattering profile is needed lateron for a model fit and should be as accurate as possible. For increasing ion concentrations the scattering profiles shift to higher intensity values at low $q$-values. This is an indication for an increase in the molecular weight, which correlates with an 
(a)

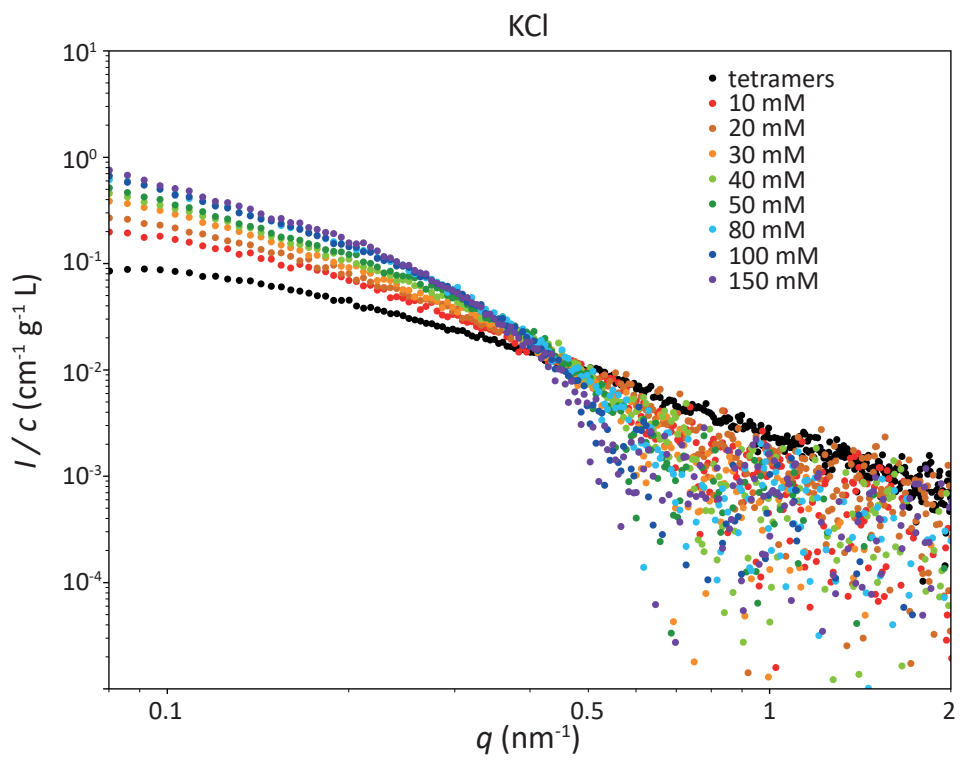

(b)

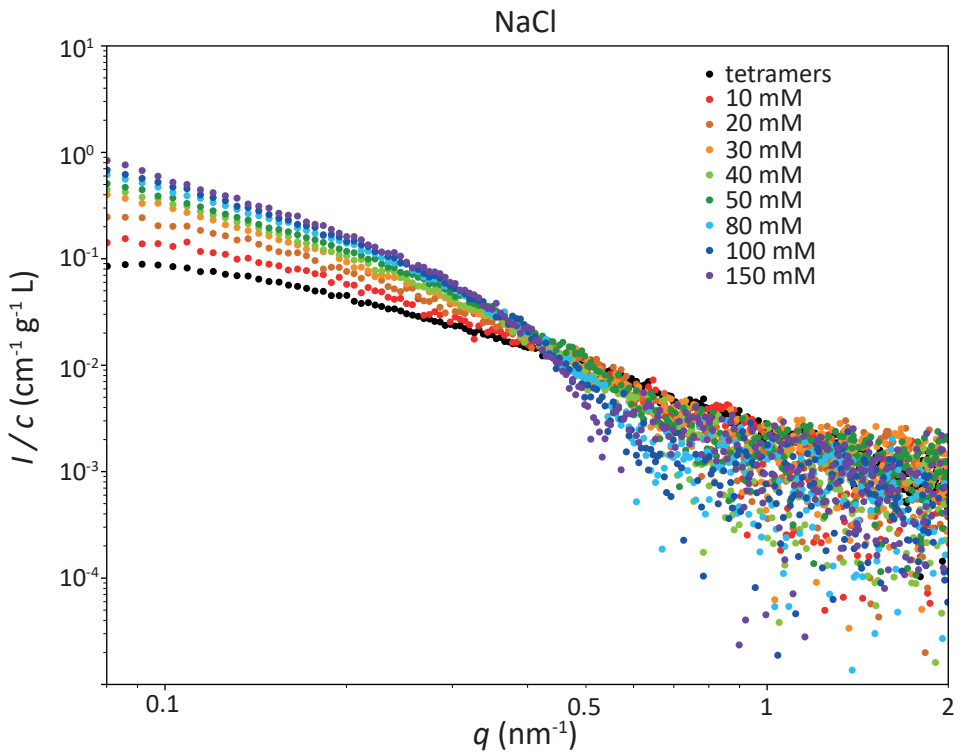

Figure 5.2: Scattering profiles of vimentin assembled with monovalent ions at various ion concentrations. Ion concentrations ranged from $10 \mathrm{mM}$ to $150 \mathrm{mM}$ for (a) $\mathrm{KCl}$ and (b) $\mathrm{NaCl}$. The black curve corresponds to vimentin without ions added for assembly. The higher the ion concentration is, the higher the intensity is at low q-values and the steeper the scattering profiles are.

increase of subunits in the filament. Furthermore, the curves steepen with higher KCl concentration. A very similar behavior is observed with vimentin protein assembled with $\mathrm{NaCl}$ (Fig. 5.2b). For both ions, the Guinier analysis (Equation: 2.27) is performed. Additionally, the first and second derivative are calculated to retrieve the steepness as well as the curvature; both indicators for an increase in the filament radius. A larger negative steepness hints that the curve is steeper, indicating that the radius is increased. An increase in the curvature is also an indication that 
the radius increases. The mean slope is calculated from the whole curve whereas the mean curvature is calculated in the range from $q=0.2-0.5 \mathrm{~nm}^{-1}$. Results for Guinier analysis and the derivatives are shown in Fig. 5.3.

(a)

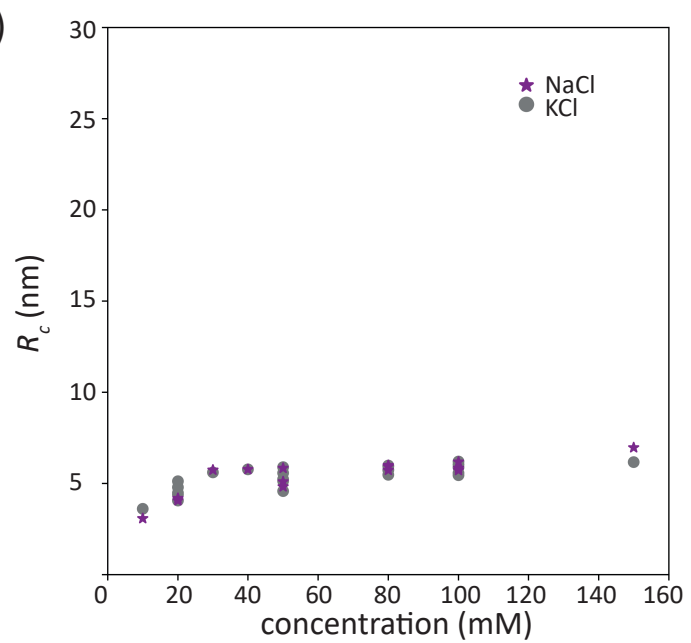

(c)

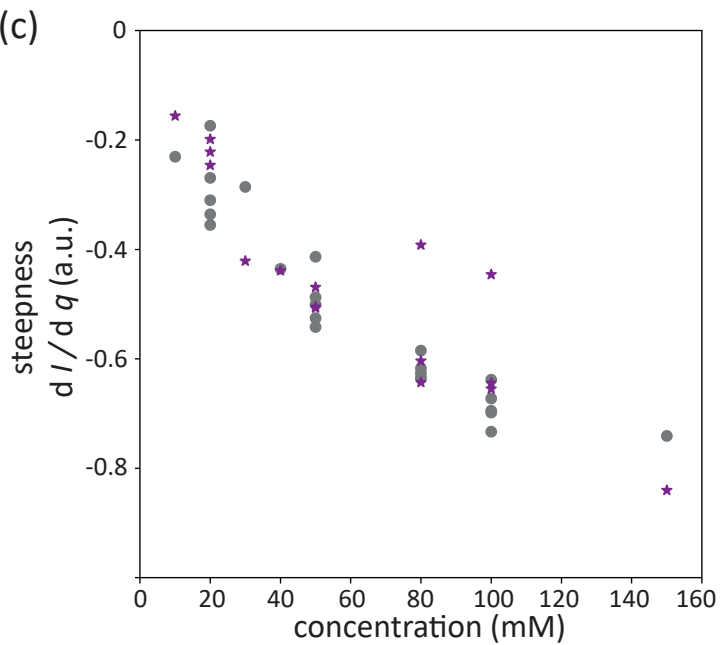

(b)

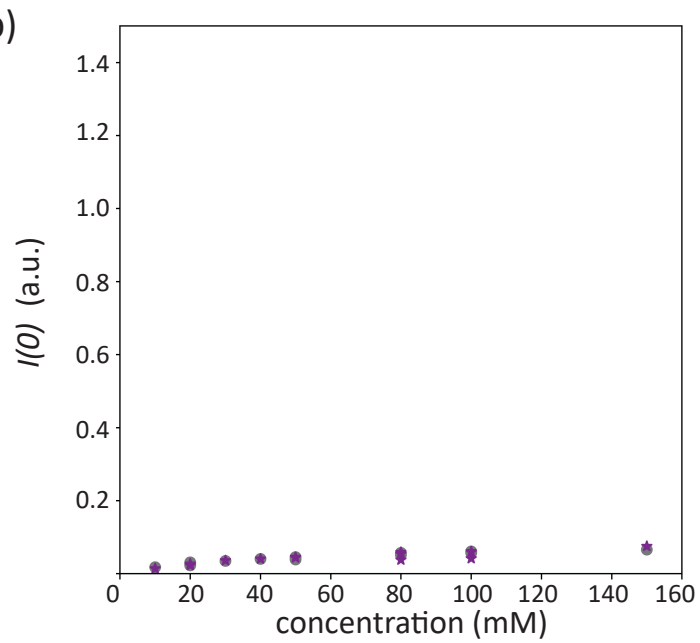

(d)

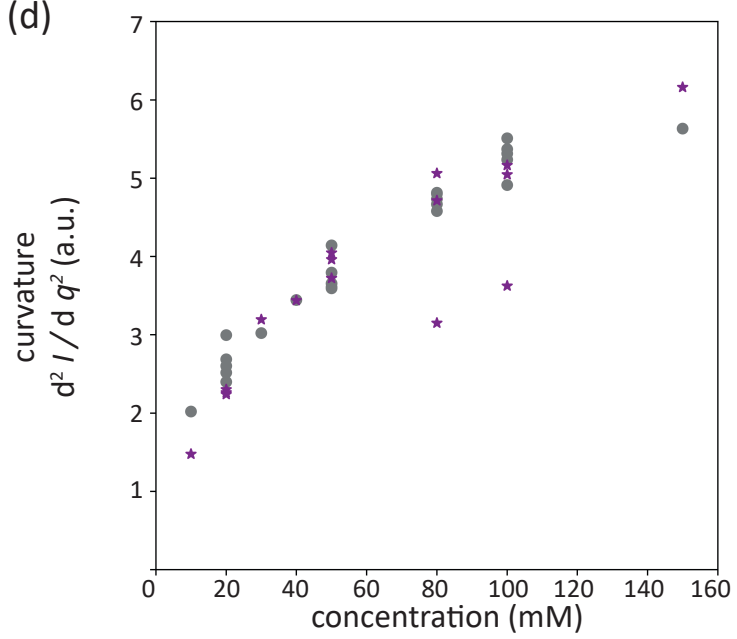

Figure 5.3: Guinier analysis of the scattering profiles for vimentin filaments assembled with monovalent ions. Analysis is performed and the (a) radius of gyration as well as the (b) $I(0)$ values are retrieved. Both, the $R_{c}$ and $I(0)$ values increase with increasing ion concentration. (c) Analysis of the first derivative of the whole curve gives information about the mean steepness. With increasing ion concentration the steepness of the curves for vimentin protein assembled with monovalent ions decreases. (d) With the second derivative the mean curvature can be calculated. For vimentin filaments assembled with monovalent ions the curvature increases with increasing ion concentrations.

The values retrieved from the Guinier analysis are shown in Table 5.1. For concentrations, where multiple measurements are performed, the arithmetic mean of the retrieved $R_{c}$ and $I(0)$ values are calculated.

Analysis of the radius of gyration (Fig. 5.3a and Table 5.1) shows that $R_{c}$ increases with increas- 
Table 5.1: Results of the Guinier analysis of vimentin assembled with KCl/NaCl at different concentrations. The calculated $R_{c}$ and $I(0)$ values are listed.

\begin{tabular}{lll}
\hline Ion concentration $(\mathrm{mM})$ & $R_{c}(\mathrm{~nm})$ & $I(0)$ (a.u.) \\
\hline 10 & $3.60 / 3.09$ & $0.018 / 0.014$ \\
20 & $4.26 / 4.14$ & $0.026 / 0.024$ \\
30 & $5.61 / 5.74$ & $0.035 / 0.036$ \\
40 & $5.77 / 5.78$ & $0.041 / 0.040$ \\
50 & $5.29 / 5.25$ & $0.043 / 0.045$ \\
80 & $5.78 / 5.89$ & $0.055 / 0.050$ \\
100 & $5.83 / 5.92$ & $0.059 / 0.054$ \\
150 & $6.18 / 6.96$ & $0.067 / 0.075$ \\
\hline
\end{tabular}

ing ion concentration, however the increase is very small from ion concentrations of $30 \mathrm{mM}$ on. No differences between the values retrieved for vimentin assembled in the presence of either $\mathrm{KCl}$ or $\mathrm{NaCl}$ can be observed. The $I(0)$ values increase with increasing ion concentration. Calculating the first derivative of the scattering curve provides information about the steepness of the scattering profile. With increasing ion concentration the values retrieved for the mean steepness get more negative (Fig. 5.3c), which is already evident in the scattering profiles (Fig. 5.2). For vimentin assembled with both monovalent ions, the same trend can be observed, and similar values are calculated. Calculating the second derivative (curvature) the values extracted get more positive for higher ion concentrations, indicating that the curvature of the scattering curves increase at higher ion concentrations. Similar values are calculated for vimentin assembled with $\mathrm{NaCl}$ and $\mathrm{KCl}$. The mean steepness as well as the mean curvature show that with increasing ion concentration the radius of the filament increases. These findings are in agreement with the Guinier analysis.

The SAXS data is further analyzed by fitting a model for cylindrical micelles to the data (see Section 3.3.4) $[2,16,17]$. From the analysis the radius of the cylindrical core, as well as the radius of gyration of the side chains, in our case the tails of the monomers, can be retrieved. When fitting the model to the data, the model introduced by Pedersen [16] is extended by a tetramer term as already performed by Brennich et al. [2]. By including this term in the micelle model, it is accounted for tetramers in the solution, which can contribute to the scattering signal. Thus, next to the radius $(R)$ and radius of gyration of the tails $\left(R_{g}\right)$, the amount of tetramers in the solution can be retrieved. An example of a fit to the data is shown for vimentin assembled with $20 \mathrm{mM} \mathrm{NaCl}$ in Fig. 5.4.

In the used fitting range from $q=0.08$ to $q=1$, the three measured scattering profiles (three different experiments) of vimentin assembled with $20 \mathrm{mM} \mathrm{NaCl}$ are very similar and no large deviations are observed. Fitting the model to the data, the fit describes the data well in the chosen range. All data fits are shown in the Appendix B. The radius $R$ and the radius of gyra- 


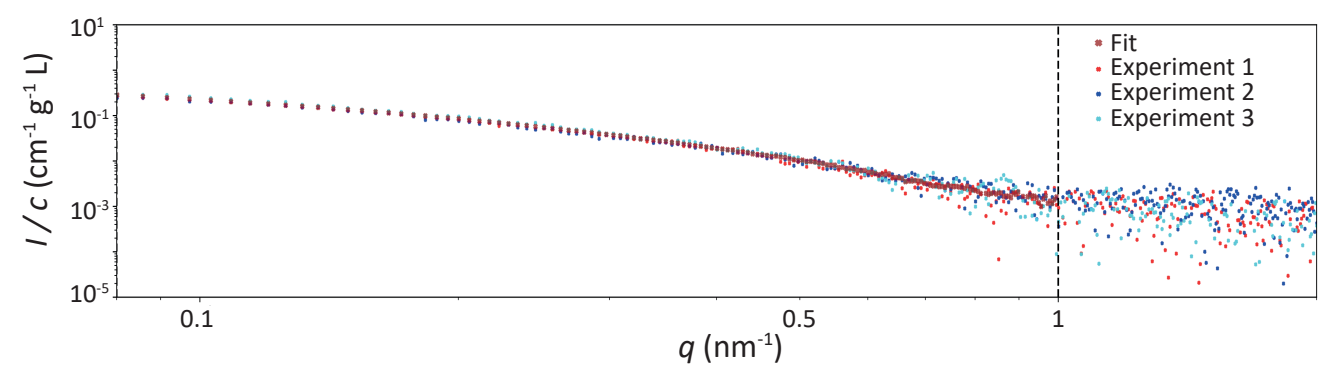

Figure 5.4: Scattering profiles of vimentin assembled with $20 \mathrm{mM} \mathrm{NaCl}$ and the respective fit. Using the micelle model introduced by Pedersen [16] and extended by a term to include tetramers in the system the data can be fitted. The fit describes the data well.

tion of the tails $R_{g}$ from vimentin filaments assembled with $\mathrm{KCl}$ and $\mathrm{NaCl}$ are shown in Fig. 5.5. Whereas the earlier introduced $R_{c}$ value is the radius of gyration of the filament with the tails, the calculated radius of gyration $R_{g}$ from the model describes the radius of gyration of the tails.

(a)

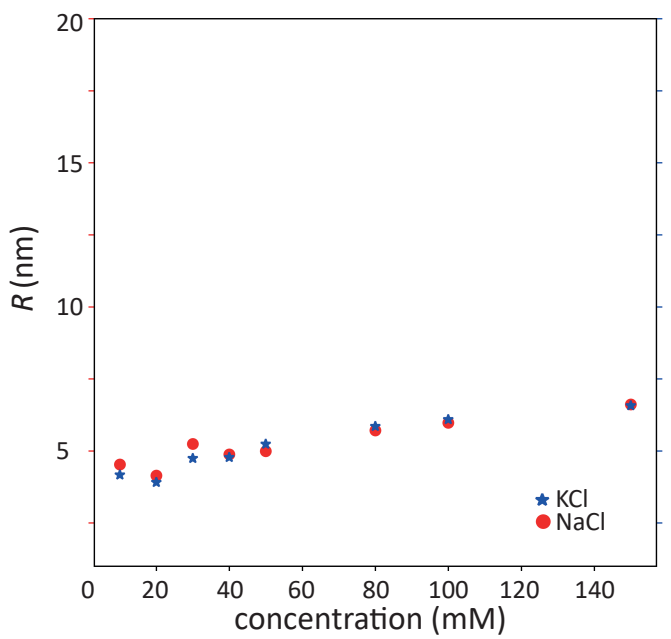

(b)

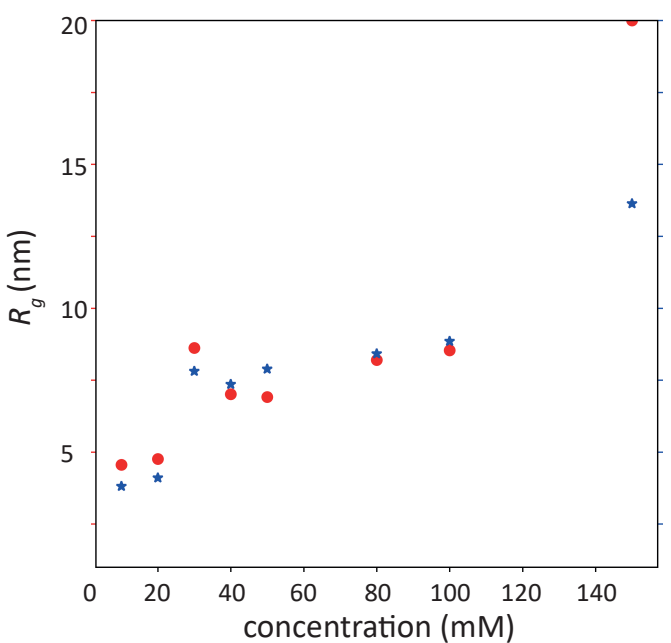

Figure 5.5: Analysis of vimentin assembled with $\mathrm{KCl}$ or $\mathrm{NaCl}$ at different concentrations using a model for micelles. (a) The retrieved radii of the filaments get larger with increasing ion concentration. (b) The calculated radii of gyration of the tails protruding from the filament increase with increasing ion concentrations. Similar results are found for vimentin protein assembled with both monovalent ions.

For both monovalent ions the radius of the vimentin filament increases with increasing ion concentration and the values retrieved are similar for vimentin assembled in the presence of $\mathrm{KCl}$ or $\mathrm{NaCl}$. Whereas the radius of the filament is roughly $4 \mathrm{~nm}$ when assembled with $10 \mathrm{mM}$ monovalent salt, it is around $6.5 \mathrm{~nm}$ when assembled in the presence of $150 \mathrm{mM} \mathrm{KCl} / \mathrm{NaCl}$. The radius of gyration of the tails $R_{g}$ has a similar value when vimentin is assembled with either $\mathrm{NaCl}$ or $\mathrm{KCl}$. Like the radius, the radius of gyration increases with increasing ion concentration. A larger variance between the retrieved $R_{g}$ values is observed at an ion concentration of $150 \mathrm{mM}$, where $R_{g}$ found for vimentin assembled with $\mathrm{NaCl}$ is larger than what is found when vimentin is as- 
sembled in the presence of $\mathrm{KCl}$. Indeed, it is not possible to retrieve the radius of gyration for vimentin filaments assembled with $150 \mathrm{mM} \mathrm{NaCl}$, as a value of $20 \mathrm{~nm}$ is set as a limit of the fitting range. Regarding the fit for vimentin assembled with $150 \mathrm{mM} \mathrm{NaCl}$ describes the data well, indicating that there might be more than one optimal solution to fit the data (see Appendix B). The tetrameric contribution in both cases shows a decrease of the amount of tetramers in the solution with increasing ion concentration (Fig. 5.6).

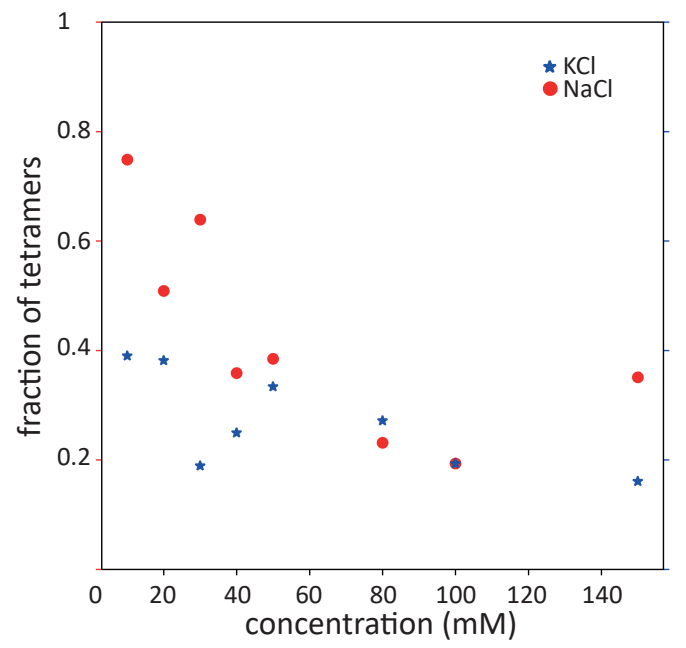

Figure 5.6: Fraction of tetramers in the measured solution for vimentin protein assembled with monovalent ions. For vimentin assembled with both monovalent ions the amount of tetramers in the system reduces with increasing ion concentrations.

At low ion concentrations the amount of tetramers in the solution is lower for vimentin filaments assembled with $\mathrm{KCl}$ than for vimentin filaments assembled with $\mathrm{NaCl}$. For vimentin assembled with $150 \mathrm{mM} \mathrm{NaCl}$ the amount of tetramers in the solution increases again, which is surprising. However as the fit failed to retrieve the $R_{g}$ value properly at this ion concentration, it could mean that the fit parameters are not optimally chosen. Comparing the values from the Guinier analysis with the model-based radii for the filaments $(R)$, the values are very similar. In addition to SAXS measurements, microscopy experiments are performed. As with SAXS only the radius of vimentin filaments can be investigated, microscopy is performed to see if single filaments, bundles or networks are formed. Fluorescence microscopy images of vimentin wt mixed with vimentin C385A fluorescently labeled with ATTO647N and assembled with $100 \mathrm{mM}$ $\mathrm{KCl}$ or $100 \mathrm{mM} \mathrm{NaCl}$ are taken (Fig. 5.7).

In both cases filaments are formed when assembling vimentin protein with $100 \mathrm{mM} \mathrm{KCl}$ or $\mathrm{NaCl}$. Single filaments are present when assembling with both monovalent ions and the filaments are comparable to the filaments in the AFM images shown in Chapter 4. 
(a)

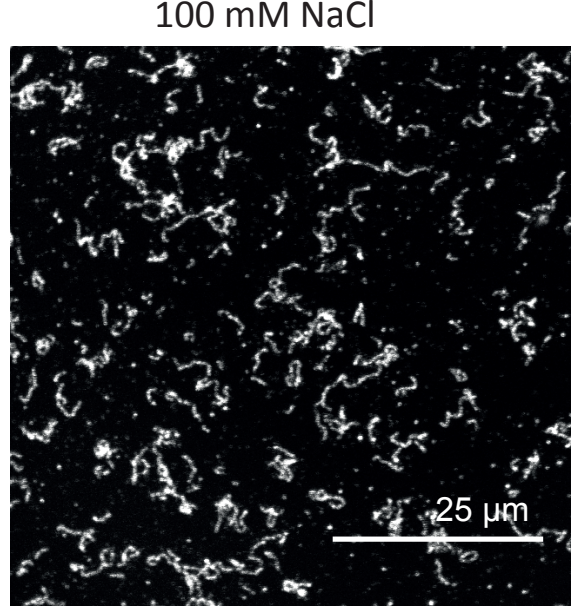

(b)

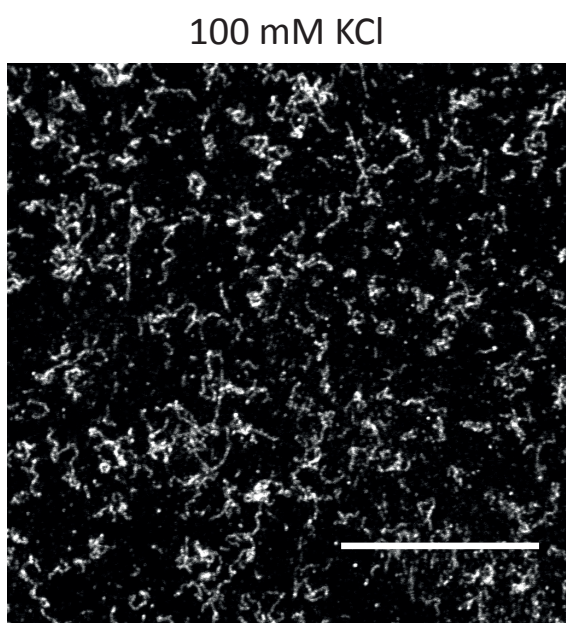

Figure 5.7: Fluoresence microscopy images of vimentin assembled with monovalent ions. Vimentin is assembled with (a) $100 \mathrm{mM} \mathrm{NaCl} \mathrm{and} \mathrm{(b)} 100 \mathrm{mM} \mathrm{KCl}$. In both cases, single filaments are formed.

\subsection{Vimentin Filaments Assembled in the Presence of Divalent Ions}

Beside monovalent ions, the assembly of vimentin in the presence of divalent ions is investigated as well. $\mathrm{Mg}^{2+}$ and $\mathrm{Ca}^{2+}$ are chosen, as those are the most abundant divalent ions in the cell, next to $\mathrm{Zn}^{2+}[1,18]$. Different concentrations in the range from $0.5 \mathrm{mM}$ to $10 \mathrm{mM}$ are measured and the scattering profiles are shown in Fig. 5.8. The range is chosen according to what was already measured by Brennich et al. for $\mathrm{MgCl}_{2}$ [2] and is expanded to higher ion concentrations to see if there is a threshold for precipitation (aggregation to very dense networks) of vimentin filaments. For both ions the same concentrations are measured for better comparison. The scattering profiles of vimentin filaments assembled in the presence of $\mathrm{CaCl}_{2}$, increase with increasing ion concentration at low $q$-values and the data recorded at lower ion concentration look similar in shape to the ones measured with monovalent ions. However, after a concentration of $4 \mathrm{mM}$ on, the curves change drastically. The rather flat part of the curve at low $q$-values vanishes and the curve follows a decrease. The change in the scattering profile indicates that the protein changed its conformation. Already when filling the capillaries, several solutions precipitated immediately ( $4 \mathrm{mM}, 5 \mathrm{mM}, 10 \mathrm{mM} \mathrm{CaCl}_{2}$ ). This matches well to the observed changed scattering profiles. Looking at the scattering profiles recorded for $\mathrm{MgCl}_{2}$, it can be observed that only for $10 \mathrm{mM} \mathrm{MgCl}_{2}$ the scattering curve looks atypical for vimentin filaments. Again, this capillary showed precipitation of vimentin. Already from these results it can be concluded that both divalent ions interact differently with vimentin. For both ions, the Guinier analysis and the derivatives are calculated and the results are shown in Fig. 5.9 and Table 5.2. 
(a)

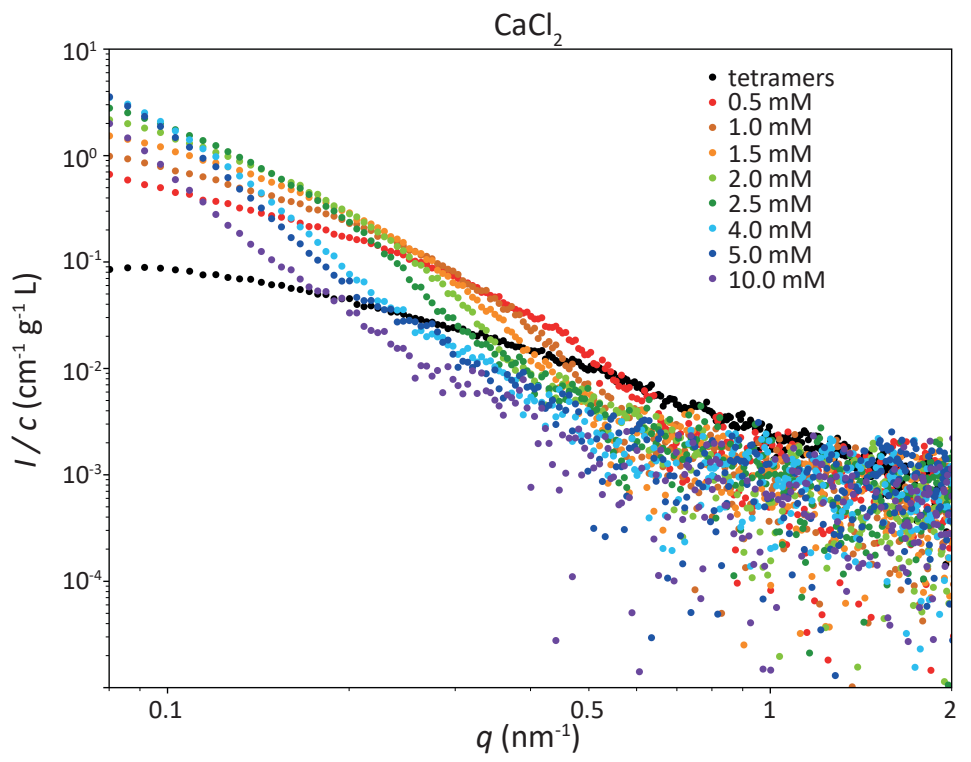

(b)

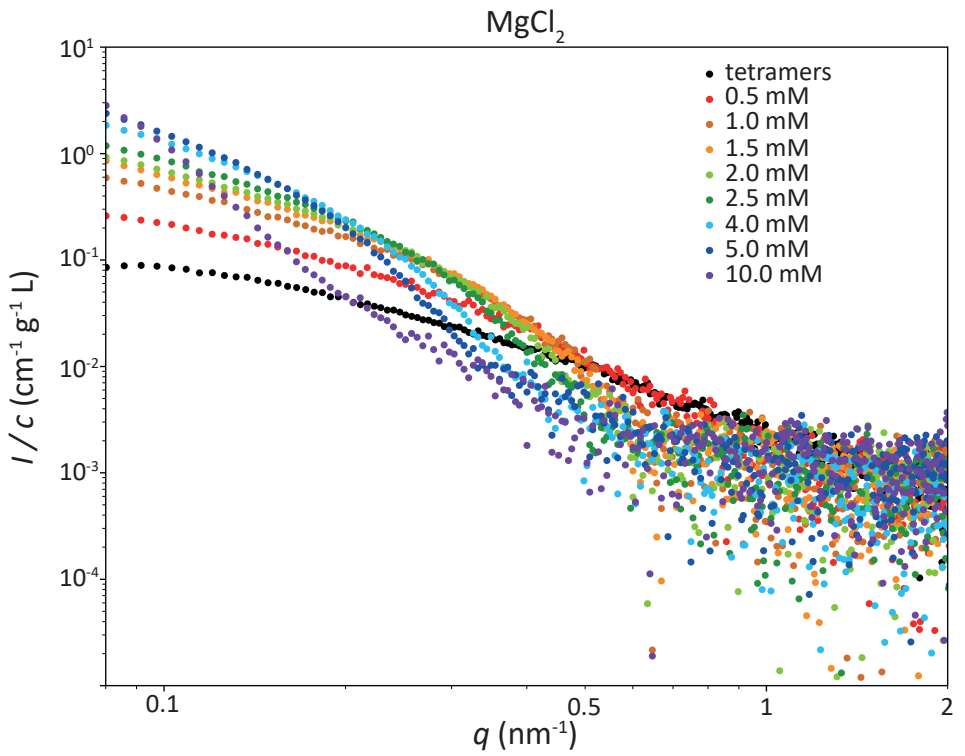

Figure 5.8: Scattering profiles of vimentin assembled with divalent ions at eight different concentrations. Ion concentrations ranged from $0.5 \mathrm{mM}$ to $10 \mathrm{mM}$ for both investigated ion species. The black curve corresponds to vimentin without any ions added for assembly. (a) Scattering profiles of vimentin assembled with $\mathrm{CaCl}_{2}$. For $\mathrm{CaCl}_{2}$ concentrations of $4 \mathrm{mM}$ and higher, the scattering profiles change the shape. For those three ion concentrations vimentin precipitated. (b) SAXS curves for vimentin assembled with $\mathrm{MgCl}_{2}$. At $10 \mathrm{mM}$ $\mathrm{MgCl}_{2}$ the vimentin curve looks different compared to the other measured concentrations. At this concentration vimentin precipitated.

All data points where visual inspection of the capillaries indicated precipitation are marked with green circles. Guinier analysis is no longer valid in precipitated samples, as for Guinier analysis, the particles have to be dilute, which is not the case in an precipitated sample. Data of precipitated vimentin is still analyzed for completeness, however will not be discussed any further. 
(a)
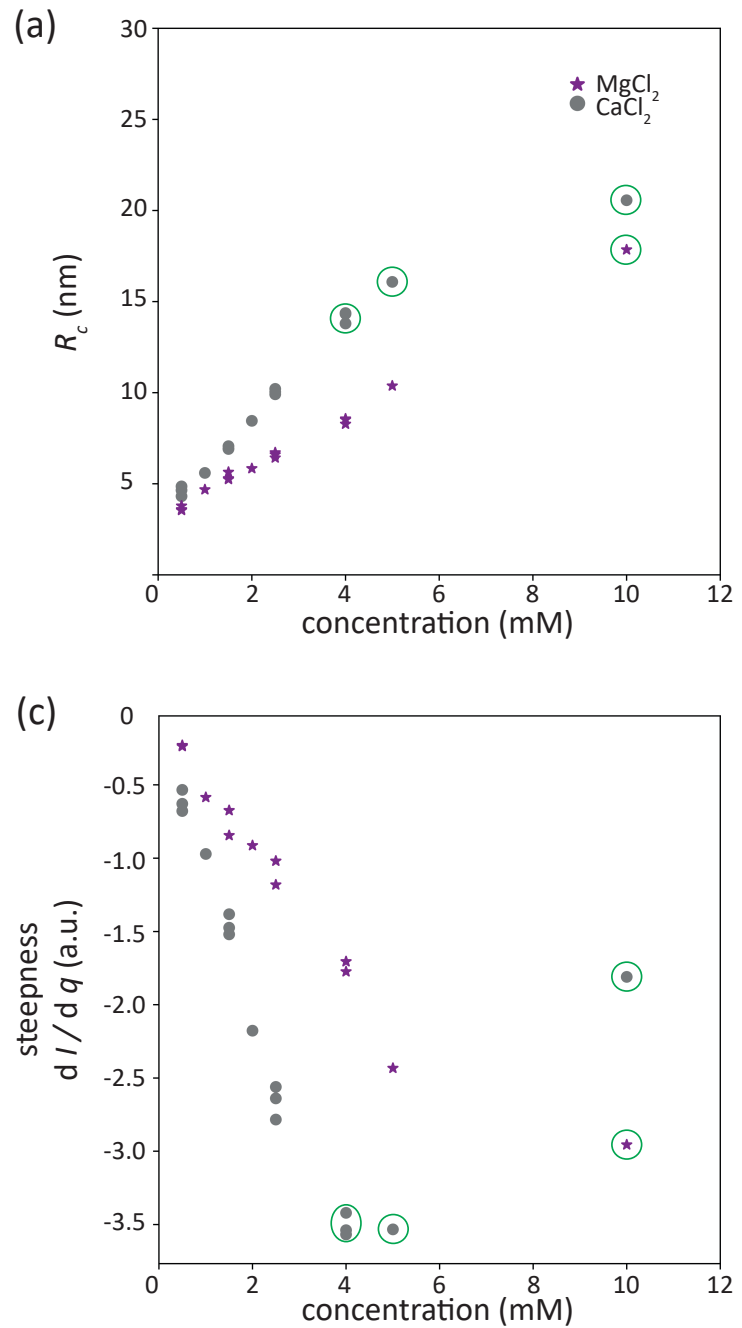

(b)
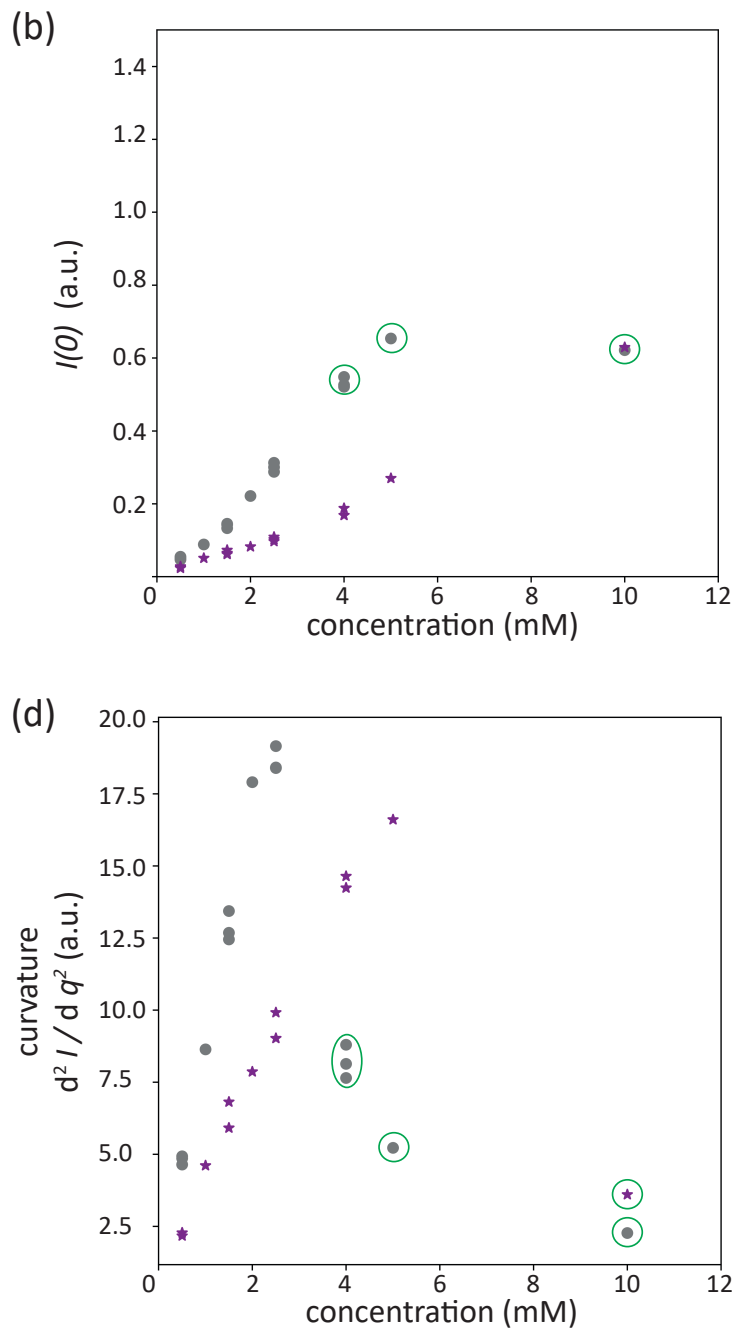

Figure 5.9: Guinier analysis of vimetnin filaments assembled with $\mathrm{CaCl}_{2}$ and $\mathrm{MgCl}_{2}$. (a) The radius of gyration is calculated. With increasing ion concentration $R_{c}$ is increasing. (b) $I(0)$ values are retrieved from Guinier analysis. For both ions, the I(0) values increase. (c) Analysis of the first derivative of the whole curve provides information about the mean slope. Stronger negative values are retrieved for higher ion concentrations. However, the precipitated vimentin, assembled with $10 \mathrm{mM} \mathrm{CaCl} 2$, does not follow this trend. (d) The mean curvature is calculated. Higher values for the curvature are retrieved for higher ion concentrations. An exception are the precipitated samples, where the curvature is low. Measurements where precipitation is visible by eye in the capillary are marked with green circles.

The radius of gyration $\left(R_{c}\right)$ of vimentin filaments increase steadily with higher ion concentrations of $\mathrm{CaCl}_{2}$ and $\mathrm{MgCl}_{2}$ (Fig. 5.9, Table 5.2). The $R_{c}$ values seem, however, smaller for $\mathrm{MgCl}_{2}$ than for $\mathrm{CaCl}_{2}$. This is the case when comparing the values at each ion concentration individually. However, the threshold for precipitation is lower for $\mathrm{CaCl}_{2}$ than for $\mathrm{MgCl}_{2}$. The last ion concentration where vimentin is not precipitated in presence of $\mathrm{CaCl}_{2}$ is $2.5 \mathrm{mM}$. At this concentration, the radius of gyration of vimentin is roughly $10 \mathrm{~nm}$. The last concentration measured 
Table 5.2: Results of the Guinier analysis of vimentin assembled with $\mathrm{CaCl}_{2} / \mathrm{MgCl}_{2}$ at different concentrations. The calculated $R_{c}$ and $I(0)$ values are shown. Aggregated vimentin samples are marked with *

\begin{tabular}{lll}
\hline Ion concentration $(\mathrm{mM})$ & $R_{c}(\mathrm{~nm})$ & $I(0)$ (a.u.) \\
\hline 0.5 & $4.61 / 3.64$ & $0.051 / 0.025$ \\
1.0 & $5.60 / 4.68$ & $0.088 / 0.051$ \\
1.5 & $6.97 / 5.39$ & $0.140 / 0.066$ \\
2.0 & $8.45 / 5.84$ & $0.221 / 0.082$ \\
2.5 & $10.06 / 6.58$ & $0.300 / 0.103$ \\
4.0 & $14.17^{*} / 8.46$ & $0.532^{*} / 0.174$ \\
5.0 & $16.09^{*} / 10.38$ & $0.654^{*} / 0.270$ \\
10.0 & $20.57^{*} / 17.90^{*}$ & $0.622^{*} / 0.629^{*}$ \\
\hline
\end{tabular}

with $\mathrm{MgCl}_{2}$ where vimentin is not precipitated is $5 \mathrm{mM}$. At this ion concentration vimentin filaments exhibit a radius of gyration of $10.4 \mathrm{~nm}$, which is similar to vimentin assembled with $2.5 \mathrm{mM} \mathrm{CaCl}_{2}$. Thus, the filaments exhibit a similar radius of gyration before precipitation (formation of very dense networks) of the protein occurs. The retrieved $I(0)$ values from Guinier analysis show a similar behavior as the extracted $R_{c}$ values (Fig. 5.9b, Table 5.2). The values increase for higher ion concentrations. Comparing the $I(0)$ values at the last ion concentration where vimentin is not precipitated, the values extracted for vimentin assembled with $\mathrm{CaCl}_{2}$ are slightly larger than for vimentin filaments assembled with $\mathrm{MgCl}_{2}$.

The calculated values of the mean steepness are more negative with increasing ion concentration for both $\mathrm{CaCl}_{2}$ and $\mathrm{MgCl}_{2}$, which indicates that the radius of the filament increases. The analysis of the second derivative yields the mean curvature, which increases with increasing ion concentration. This is again an indication that the radius increases. Comparing again the last values before vimentin filaments start to precipitate, similar values for vimentin filaments assembled with $\mathrm{CaCl}_{2}$ and $\mathrm{MgCl}_{2}$ are retrieved. The analysis of the mean steepness and mean curvature is consistent with the observed increase of the $R_{c}$ with increasing ion concentration. Again, the micelle model is fit to the data measured with both divalent ions. The radius of the filament $R$ and the radius of gyration $R_{g}$ of the tails is shown in Fig. 5.10.

For both ions it is evident that the radius of vimentin increases with increasing ion concentrations. At the last ion concentration before vimentin precipitates $\left(2.5 \mathrm{mM}\right.$ for $\mathrm{CaCl}_{2}$ and $5 \mathrm{mM}$ for $\mathrm{MgCl}_{2}$ ), the radius is close to $10 \mathrm{~nm}$ in both cases. A similar trend can be observed for the radius of gyration of the tails, when vimentin filaments are assembled with $\mathrm{CaCl}_{2}$. The values retrieved for $R_{g}$ increase with increasing $\mathrm{CaCl}_{2}$ concentration. For vimentin assembled in the presence of $\mathrm{MgCl}_{2}$ there is no clear trend. The $R_{g}$ values only increase from $0.5 \mathrm{mM}$ to $1.0 \mathrm{mM}$ and then decrease again up to a salt concentration of $2.5 \mathrm{mM} \mathrm{MgCl}_{2}$. Subsequently the $R_{g}$ values increase again. At the last ion concentration before vimentin precipitates, the retrieved $R_{g}$ values are similar. It is unexpected that the $R_{g}$ values do not seem to follow a certain trend. The 
(a)

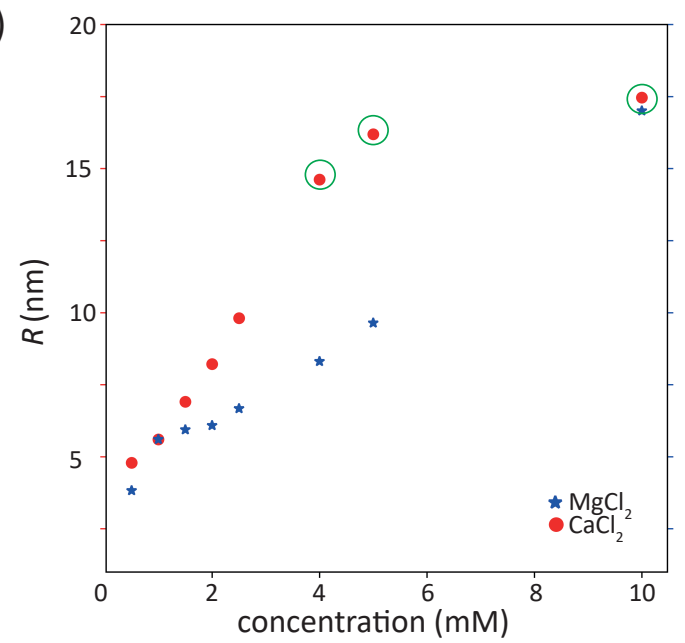

(b)

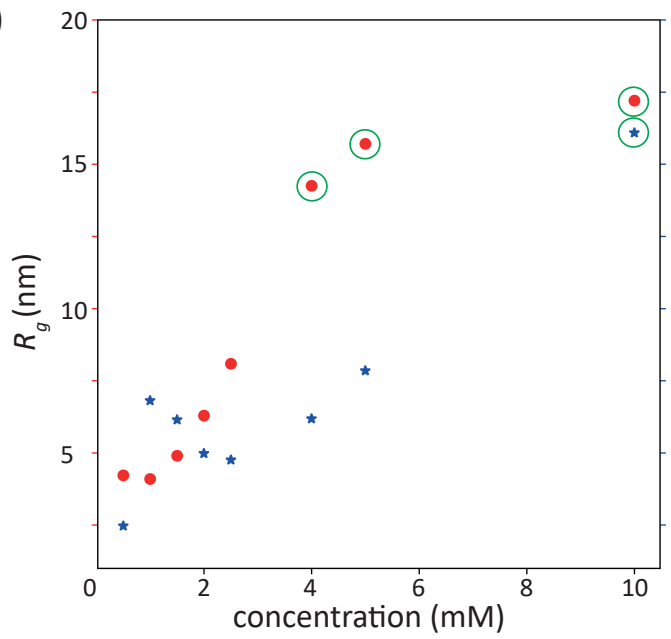

Figure 5.10: Analysis of vimentin assembled with $\mathrm{CaCl}_{2}$ and $\mathrm{MgCl}_{2}$ using a micelle model. (a) The calculated radius of the filament retrieved at the different concentrations is shown. Vimentin protein assembled with both divalent ions shows an increase of the radius of the filament with increasing ion concentration. (b) Calculated radius of gyration of the tails protruding from the filament. For vimentin protein assembled with $\mathrm{CaCl}_{2}$ the radius of gyration increases with increasing ion concentration. For vimentin filaments assembled with $\mathrm{MgCl}_{2}$ the radius of gyration first increases at low ion concentrations and decreases for vimentin filaments assembled up to an ion concentration of $2.5 \mathrm{mM}$. After that, the radius of gyration increases again. Data from precipitated vimentin is marked with green circles.

fraction of tetramers in the solution, is less than $40 \%$ for both ions at a ion concentration of $0.5 \mathrm{mM}$ and reduces to $0 \%$ for higher ion concentrations (Fig. 5.11).

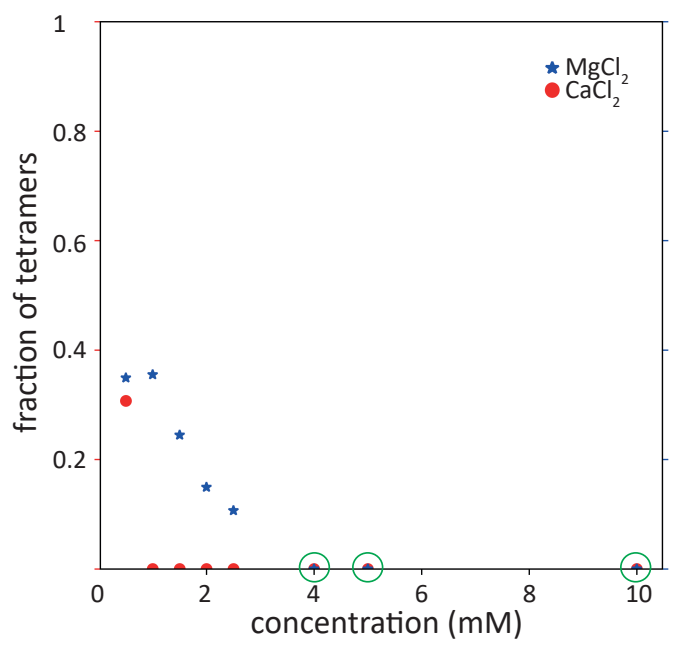

Figure 5.11: Fraction of tetramers in the measured solution for vimentin protein assembled with $\mathrm{MgCl}_{2}$ and $\mathrm{CaCl}_{2}$. For both ions, the amount of tetramers in the system reduces with increasing ion concentration. For vimentin assembled with $\mathrm{MgCl}_{2}$ the amount of tetramers in the solution is reduced to zero at an ion concentration of $4 \mathrm{mM}$. For vimentin filaments assembled with $\mathrm{CaCl}_{2}$ the fraction of tetramers in the system is reduced to zero at a $\mathrm{CaCl}_{2}$ concentration of $1 \mathrm{mM}$. 
In the case of $\mathrm{CaCl}_{2}$ the fraction of tetramers decreases to zero at an ion concentration of $1.0 \mathrm{mM}$. For vimentin samples assembled with $\mathrm{MgCl}_{2}$, the amount of tetramers in the solution decreases with increasing ion concentration and reduces to zero at $4.0 \mathrm{mM} \mathrm{MgCl}_{2}$. At higher ion concentrations differences between the data and the fit can be observed (see Appendix B. An exemplary fit of vimentin assembled with $4 \mathrm{mM} \mathrm{MgCl}_{2}$ is shown in Fig. 5.12.

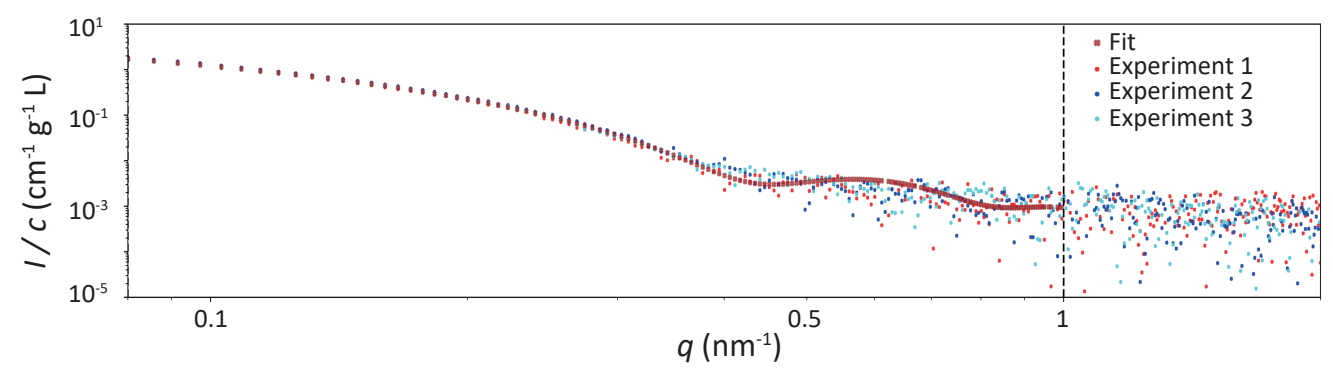

Figure 5.12: Scattering profiles of vimentin assembled by $4 \mathrm{mM} \mathrm{MgCl}_{2}$ and the respective fit. The micelle model introduced by Pedersen et at.[16], extended with an additional term to include tetramers is used to fit the data. The model describes the data at low q-values, however fails to describe the data at higher q-values.

The beginning of the curve is fitted well, however, at larger $q$-values the fit clearly shows a defined minima followed by a maxima, which cannot be observed in the data. When looking at the fits in the Appendix B it can be observed that the fits do not represent the data, from concentrations of $2 \mathrm{mM} \mathrm{CaCl}_{2}$ and $4 \mathrm{mM} \mathrm{MgCl}_{2}$ on. Comparing the retrieved $R_{c}$ values from the Guinier analysis with the radii of the filament from the model based analysis, similar values are detected.

Additionally, fluorescence microscopy images of vimentin assembled with both divalent ions are taken, to observe if single filaments or networks are present. As vimentin has different precipitation thresholds for the divalent ions, assembly is not performed at the same concentration. Vimentin assembled with $2 \mathrm{mM} \mathrm{CaCl}_{2}$ and with $4 \mathrm{mM} \mathrm{MgCl}_{2}$ are imaged (Fig. 5.13).

A drastic contrast between the effect of monovalent ions and divalent ions is observed. Vimentin assembled with divalent ions clearly shows network characteristics where vimentin filaments are cross-linked. They are no longer present as single filaments over the whole field of view, but cluster together. The networks seem larger in size for vimentin assembled with $\mathrm{MgCl}_{2}$ than with $\mathrm{CaCl}_{2}$. 
(a)

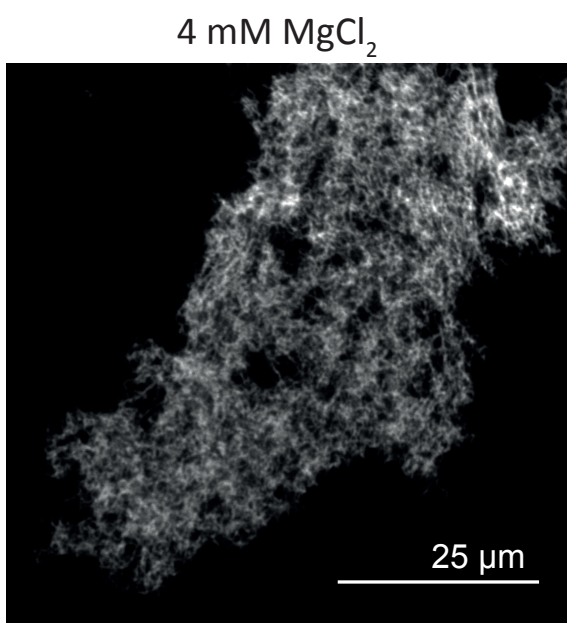

(b)

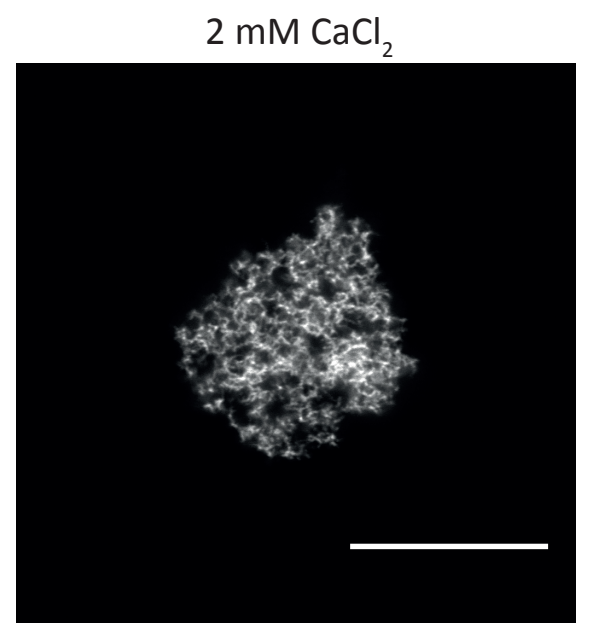

Figure 5.13: Fluorescent microscopy images of vimentin assembled with divalent ions. (a) Vimentin is assembled with $4 \mathrm{mM} \mathrm{MgCl}_{2}$. A large cluster of a vimentin network is observed (b) When assembling vimentin with $2 \mathrm{mM} \mathrm{CaCl}_{2}$, vimentin filaments form networks as well.

\subsection{Vimentin Filaments Assembled in the Presence of Hexammine-cobalt(III) Chloride}

With the trivalent salt hexammine-cobalt(III) chloride the assembly of vimentin is investigated as well. Ion concentrations in a range from $0.01 \mathrm{mM}$ to $0.5 \mathrm{mM}$ are chosen for the SAXS experiments (Fig. 5.14).

The scattering profiles for vimentin assembled with hexammine-cobalt(III) chloride show that the curves for vimentin assembled with $0.2 \mathrm{mM}$ and $0.5 \mathrm{mM}$ have a similar behavior as the precipitated samples assembled at high $\mathrm{CaCl}_{2}$ and $\mathrm{MgCl}_{2}$ concentrations. For both hexamminecobalt(III) chloride concentrations the precipitation is confirmed by visual inspection. An increase of the intensity at low $q$-values can be observed with increasing ion concentration. The scattering curves for non-precipitated vimentin are steeper with increasing ion concentration. The data is analyzed with the Guinier analysis and the derivatives are calculated. The results are summarized in Fig. 5.15 and Table 5.3, where precipitated vimentin filaments are either marked with a green circle in the Figure or $\mathrm{a}^{*}$ in the Table. Data from precipitated vimentin is analyzed but will not be discussed, as the Guinier approximation is not valid anymore.

The radius of gyration of vimentin increases with increasing ion concentrations (Fig. 5.15a, Table 5.3). Values for the radius of gyration up to $10 \mathrm{~nm}$ are reached for non-precipitated vimentin. The $I(0)$ values retrieved from Guinier analysis show an increase with increasing ion concentrations (Fig. 5.15b, Table 5.3).

Analyzing the mean steepness, the values are more negative with increasing ion concentration. 


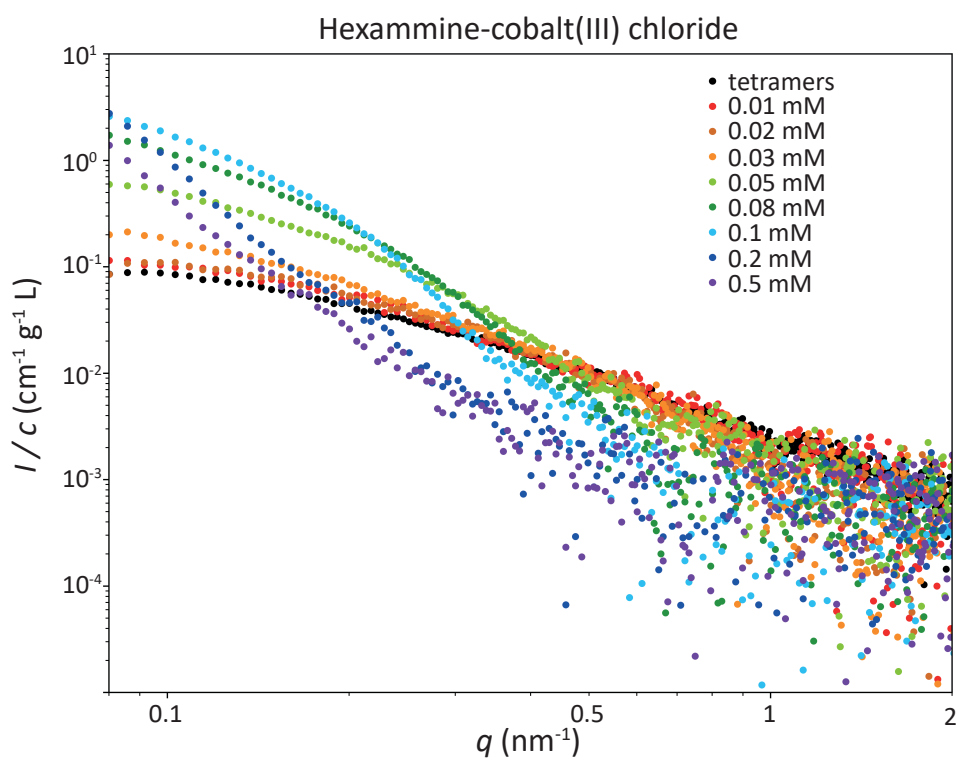

Figure 5.14: Scattering profiles of vimentin assembled with the trivalent ion hexammine-cobalt(III) chloride in the range of $0.01 \mathrm{mM}$ to $0.5 \mathrm{mM}$. At a concentration of $0.2 \mathrm{mM}$ the protein precipitated. In the scattering curves this can be seen by a change in the overall shape. For non aggregated vimentin filaments, the intensity at low q values increases with increasing ion concentration and the curves get steeper. In black the scattering curve for vimentin without any additional ions for assembly is shown.

Table 5.3: Results of the Guinier analysis of vimentin assembled with hexammine-cobalt(III) chloride at different concentrations. The calculated $R_{c}$ and $I(0)$ values are listed. Precipitated vimentin is marked with *.

\begin{tabular}{lll}
\hline Ion concentration $(\mathrm{mM})$ & $R_{\mathcal{C}}(\mathrm{nm})$ & $I(0)$ (a.u.) \\
\hline 0.01 & 3.44 & 0.013 \\
0.02 & 2.24 & 0.011 \\
0.03 & 3.91 & 0.019 \\
0.05 & 5.45 & 0.057 \\
0.08 & 8.48 & 0.172 \\
0.1 & 10.07 & 0.315 \\
0.2 & $19.79^{*}$ & $0.808^{*}$ \\
0.5 & $21.71^{*}$ & $0.300^{*}$ \\
\hline
\end{tabular}

This indicates that the radius of the vimentin filament increases with increasing ion concentration (Fig. 5.15c). Calculating the second derivative (curvature), an increase of the curvature is observed for vimentin assembled with increasing ion concentrations for non-precipitated vimentin filaments (Fig. 5.15d). This shows as well, that the radius of the filament increases with increasing ion concentration. Thus, the results gained from calculating the derivatives are in agreement with the Guinier analysis.

Applying the micelle model from Pedersen with the additional term to include tetramers to the data, yields information about the radius $R$ of the assembled filament and the radius of gyration 
(a)
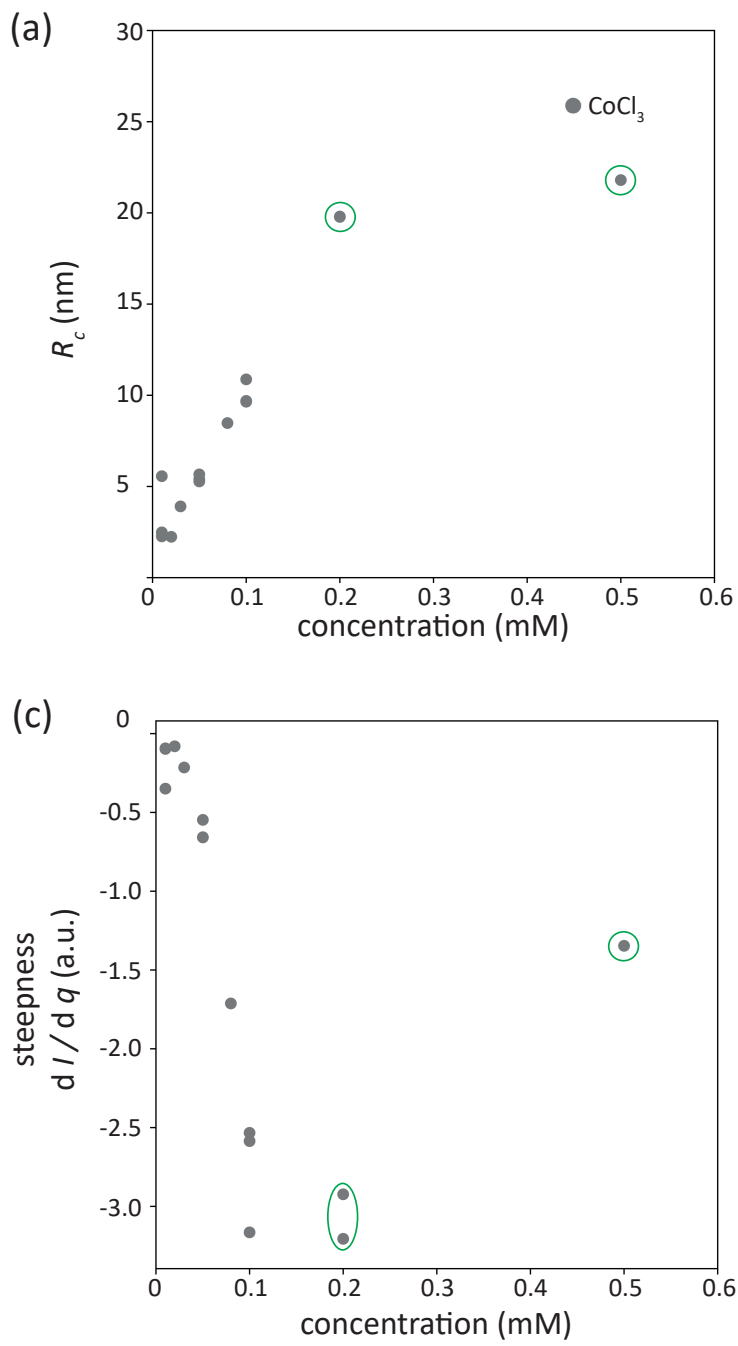

(b)

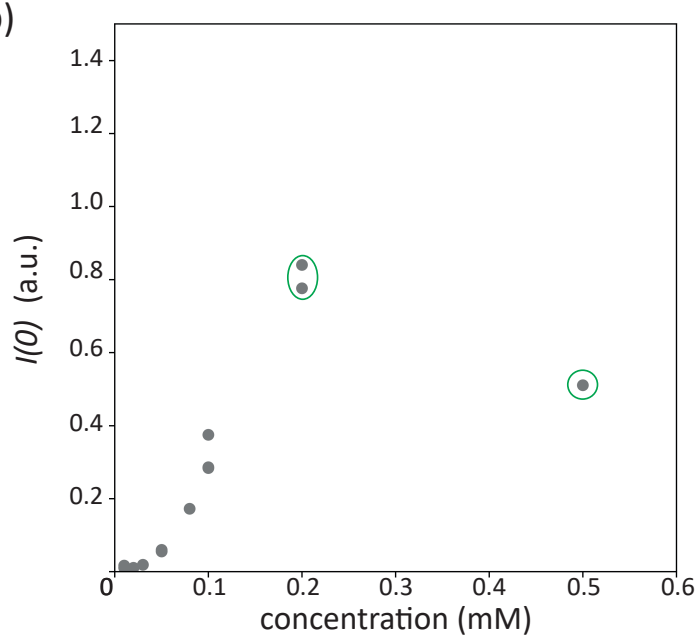

(d)

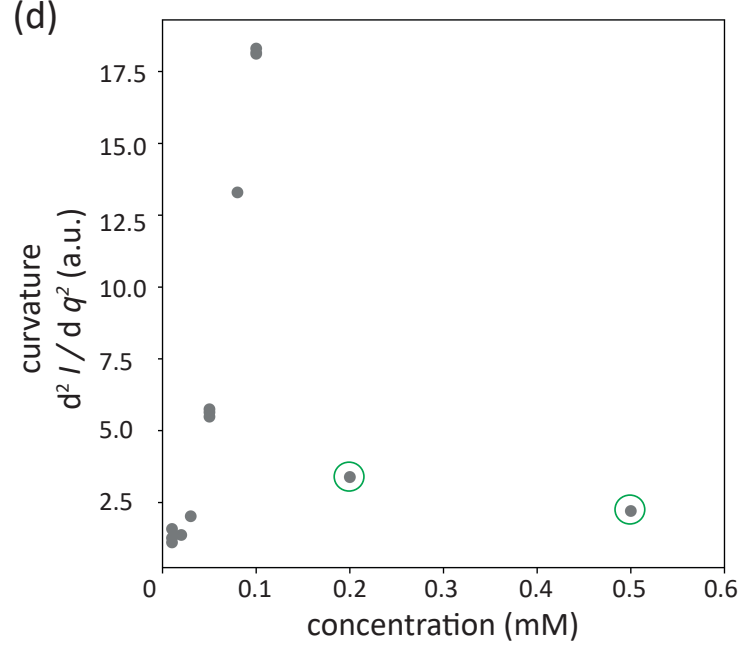

Figure 5.15: Analysis of the scattering profiles of vimentin assembled with the trivalent ion hexammine-cobalt(III) chloride. (a) From Guinier analysis, the $R_{c}$ values are calculated. With increasing ion concentration the radius increases. (b) The extracted I(0) values increase for increasing ion concentrations. (c) For increasing ion concentrations, values of the mean steepness decrease for concentrations up to $0.2 \mathrm{mM}$. (d) The curvature increases up to a concentration of $0.1 \mathrm{mM}$ hexammine-cobalt(III) chloride. Data from precipitated vimentin is marked with green circles.

of the tails $R_{g}$ (Fig. 5.16).

An overall increase of the radius of the filaments can be observed with increasing ion concentration. Data from the radius of gyration analysis follows the same overall increasing trend, however vimentin filaments assembled at low ion concentrations (up to $0.05 \mathrm{mM}$ hexamminecobalt(III) chloride), $R_{g}$ do not show a steady increase. For the amount of tetramers in the solution, first an increase from the first to the second ion concentration is observed, which is unexpected. After this increase in tetrameric contribution, the amount of tetramers in the sample decreases with increasing ion concentration and reduces to zero at an ion concentration of 
(a)

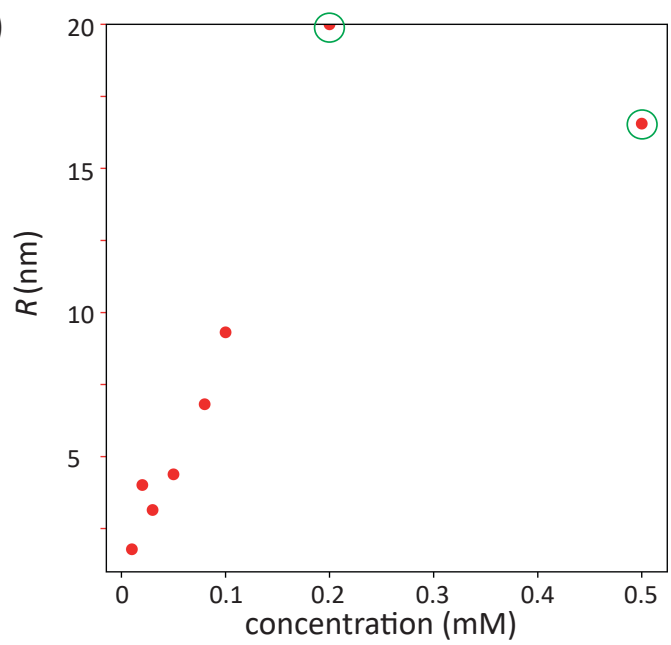

(b)

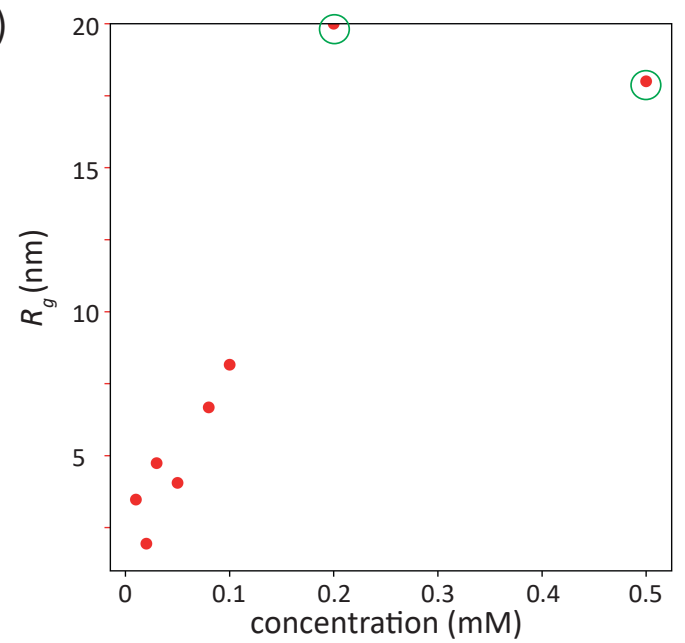

Figure 5.16: Analysis of vimentin assembled with hexammine-cobalt(III) chloride using the model. (a) Radius of the vimentin filament at different salt concentrations. The retrieved radius of the vimentin filaments increase with increasing ion concentration. (b) Calculated radii of gyration for the tails. An overall increase of the radius of gyration of the tails is found with increasing ion concentration. Data from precipitated vimentin is marked with green circles.

$0.08 \mathrm{mM}$ (Fig. 5.17).

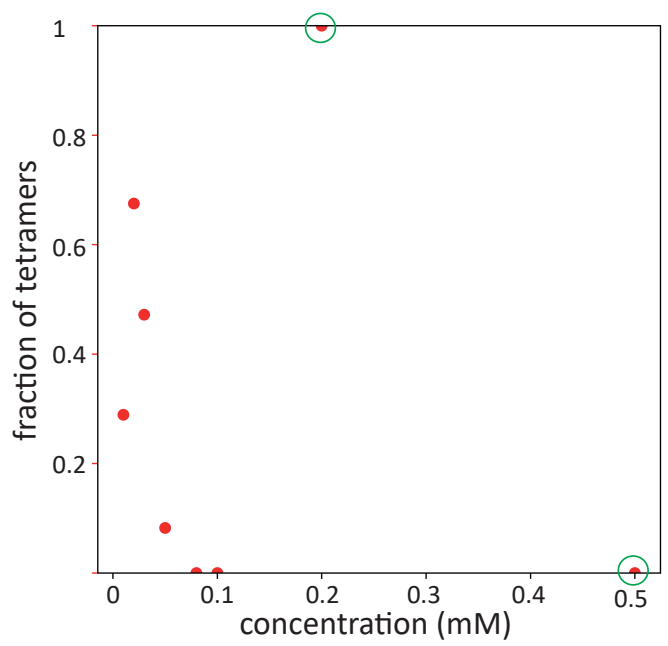

Figure 5.17: Fraction of tetramers in the measured solution for vimentin protein assembled with hexamminecobalt(III) chloride. The amount of tetramers increases between ion concentrations of 0.01 to $0.02 \mathrm{mM}$. After that, the amount of tetramers in the system decreases again until the filaments precipitate.

The amount of tetramers first increases and then decreases again. When comparing the values retrieved from Guinier analysis and the model-based analysis, slightly smaller but still similar values for the radius are found.

Again, fluorescence microscopy images are taken for vimentin assembled with $0.05 \mathrm{mM}$ 
hexammine-cobalt(III) chloride (Fig. 5.18). Assembling vimentin filaments with the trivalent ion under investigation, networks are formed like in the cases of divalent ions. Compared to networks formed with divalent ions however, the assembled vimentin networks found with hexammine-cobalt(III) chloride, are smaller in size.

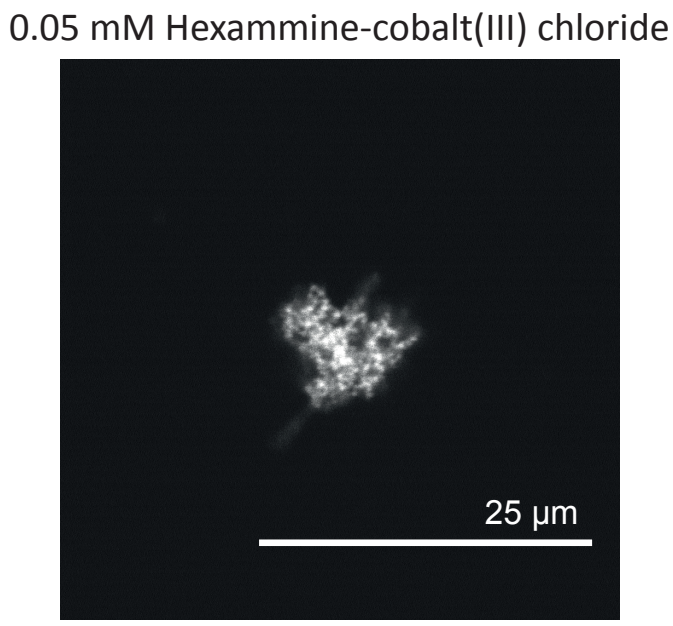

Figure 5.18: Fluorescence microscopy image of vimentin assembled with $0.05 \mathrm{mM}$ hexammine-cobalt(III) chloride. As with the divalent ions, the filaments form networks, however the networks here seem smaller in size than when vimentin is assembled in the presence of the divalent ions.

\subsection{Vimentin Filaments Assembled in the Presence of Sper- mine}

Finally, the tetravalent molecule spermine is investigated. Spermine concentrations for assembly of vimentin are chosen in a range between $0.01 \mathrm{mM}$ to $0.1 \mathrm{mM}$ and SAXS measurements are performed (Fig. 5.19).

The scattering profiles for vimentin assembled in the presence of spermine with concentrations up to $0.05 \mathrm{mM}$ show an increase of the intensity at low $q$-values and the curves become steeper with increasing ion concentrations. The last three measured concentrations $(0.08 \mathrm{mM}, 0.09 \mathrm{mM}$ and $0.1 \mathrm{mM}$ ) show an atypical scattering profile for vimentin filaments. In those measurements it is already observed by eye that the protein precipitated in the capillaries. For vimentin assembled with spermine, Guinier analysis is performed as well as the first and second derivative are calculated and the results are shown in Fig 5.20 and Table 5.4.

From Guinier analysis, the radius of gyration of the cross-section as well as the $I(0)$ values can be retrieved. For non-precipitated vimentin a radius of gyration of roughly $2.5 \mathrm{~nm}$ is found for 


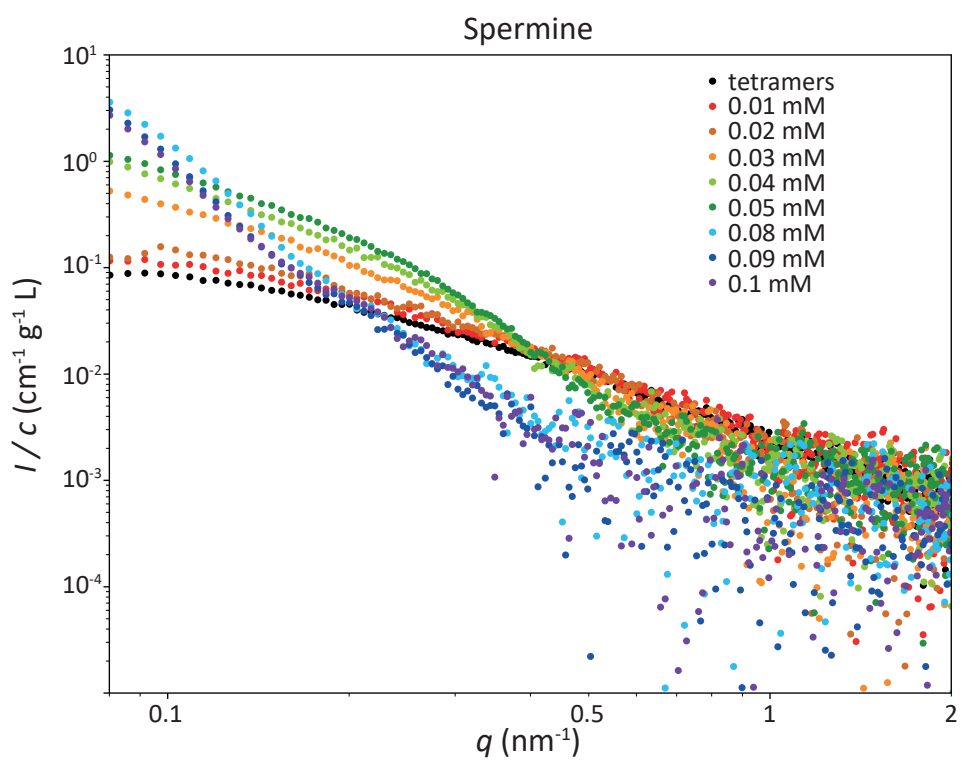

Figure 5.19: Scattering profiles of vimentin assembled with spermine. Spermine concentrations range from $0.01 \mathrm{mM}$ to $0.1 \mathrm{mM}$. Intensity values at low $q$ increases, and the curves get steeper with increasing ion concentration up to $0.05 \mathrm{mM}$ spermine. For $0.08 \mathrm{mM}$ to $0.1 \mathrm{mM}$ vimentin precipitated, which can be seen in the change of the scattering curves. In black the scattering profile of vimentin without any additional ions is shown.

Table 5.4: Results of the Guinier analysis of vimentin assembled with the tetravalent spermine at different concentrations. The calculated $R_{c}$ and $I(0)$ values are shown. Aggregated protein is marked with *.

\begin{tabular}{lll}
\hline Ion concentration $(\mathrm{mM})$ & $R_{\mathcal{C}}(\mathrm{nm})$ & $I(0)$ (a.u.) \\
\hline 0.01 & 2.32 & 0.011 \\
0.02 & 2.79 & 0.014 \\
0.03 & 6.09 & 0.045 \\
0.04 & 8.96 & 0.101 \\
0.05 & 8.03 & 0.117 \\
0.08 & $16.59^{*}$ & $0.721^{*}$ \\
0.09 & $20.11^{*}$ & $0.808^{*}$ \\
0.1 & $20.91^{*}$ & $0.848^{*}$ \\
\hline
\end{tabular}

vimentin filaments assembled by $0.01 \mathrm{mM}$ and $0.02 \mathrm{mM}$ spermine. At spermine concentrations of $0.04 \mathrm{mM}$ and $0.05 \mathrm{mM}$, a radius of roughly $8-9 \mathrm{~nm}$ is extracted for the vimentin filaments. A similar behavior is seen in the $I(0)$ values (Fig. 5.20b, Table 5.4). In Fig. 5.20c, the extracted steepness is plotted. The steepness decreases for vimentin filaments assembled with increasing ion concentrations, indicating that the radius of the filament increases. Again, it can be observed that for $0.01 \mathrm{mM}$ and $0.02 \mathrm{mM}$ similar values are retrieved. The same trend can be observed for $0.04 \mathrm{mM}$ and $0.05 \mathrm{mM}$ spermine. Calculation of the the second derivative, shows that the curvature increases for increasing ion concentrations for the non-precipitated vimentin samples. 
(a)

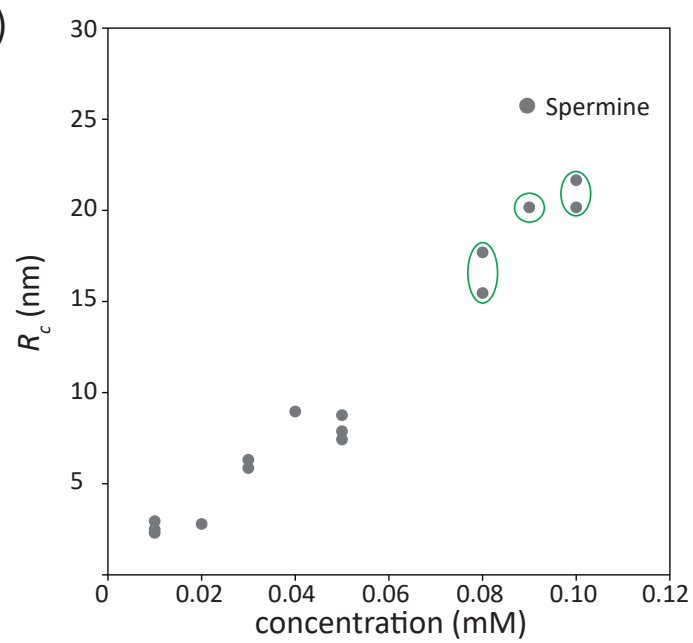

(c)

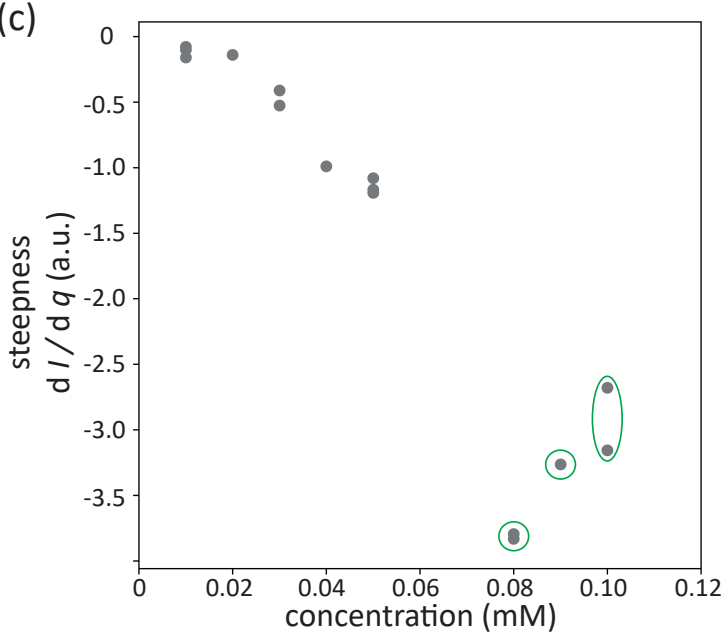

(b)

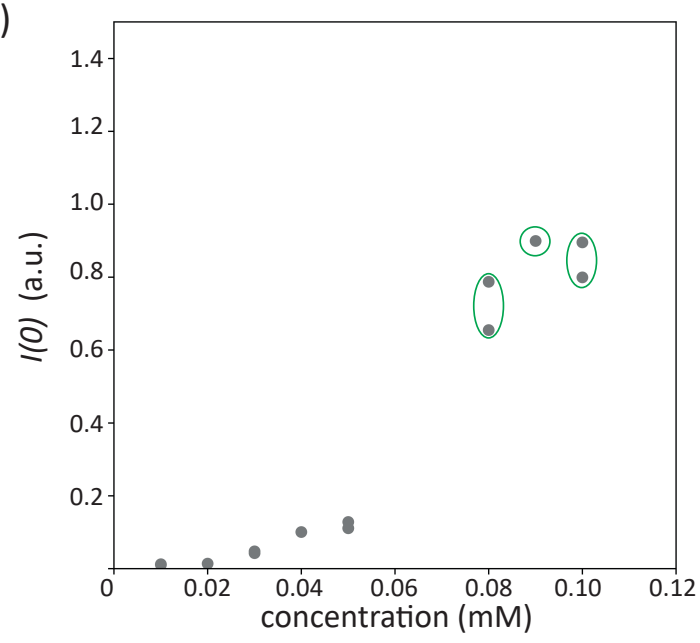

(d)

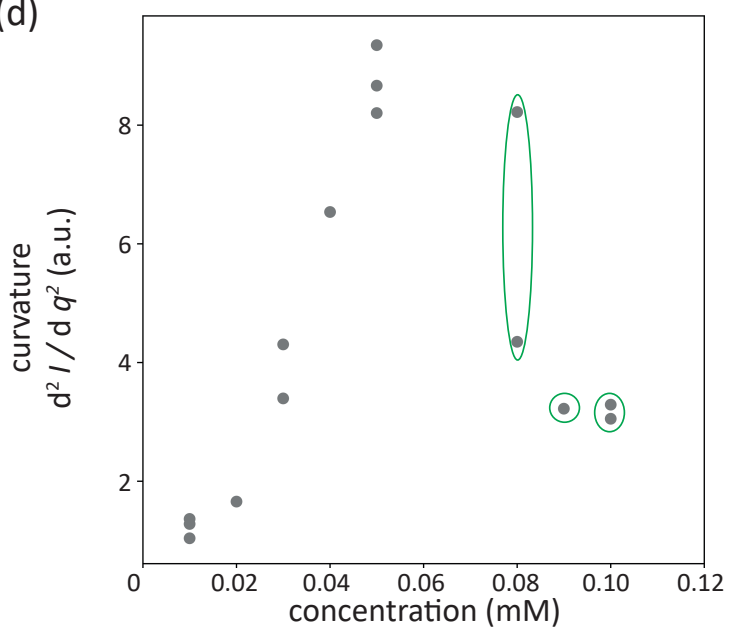

Figure 5.20: Analysis of the scattering profiles of vimentin assembled with spermine. (a) The $R_{c}$ values are calculated using Guinier analysis. With increasing ion concentration the radius increases as well. (b) The I(0) values extracted increase when increasing the spermine concentration. When assembling vimentin with $0.1 \mathrm{mM}$ spermine, the I( 0$)$ values are slightly smaller again. (c) For increasing ion concentrations the values calculated for the mean steepness get more negative for concentrations up to $0.08 \mathrm{mM}$. For a concentration of $0.09 \mathrm{mM}$ and $0.1 \mathrm{mM}$ spermine the values are less negative. (d) For the curvature the values retrieved increase up to a concentration of $0.05 \mathrm{mM}$. After that the values get smaller again. Data from precipitated vimentin is marked with green circles.

This again shows, that with increasing ion concentration the radius of the assembled filament is increasing, which is again in agreement with the Guinier analysis.

Assembling vimentin with spermine and applying the model introduced by Pedersen with the additional term for tetramers, an increase of the filament radius is observed, however, the trend is not as pronounced as for the other salts (Fig. 5.21).

Vimentin assembled at the two lowest spermine concentrations show similar filament radii. At a spermine concentration of $0.03 \mathrm{mM}$, the radius is smaller than for the lower spermine concen- 
(a)

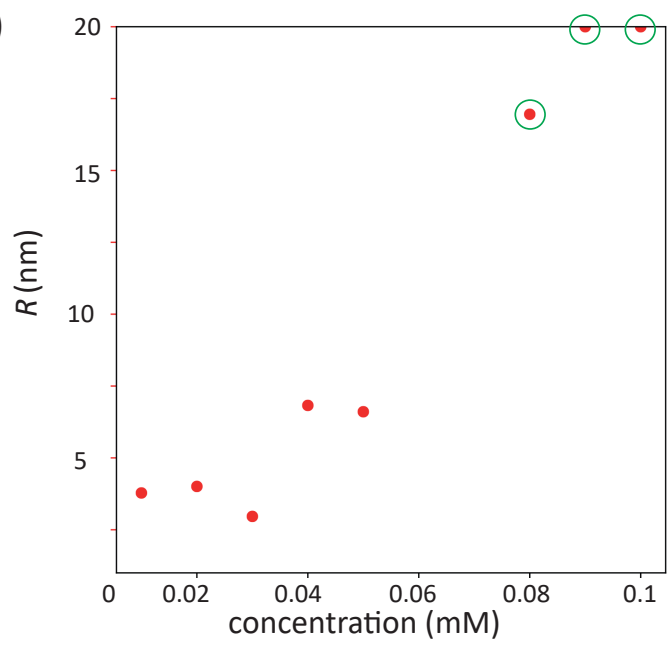

(b)

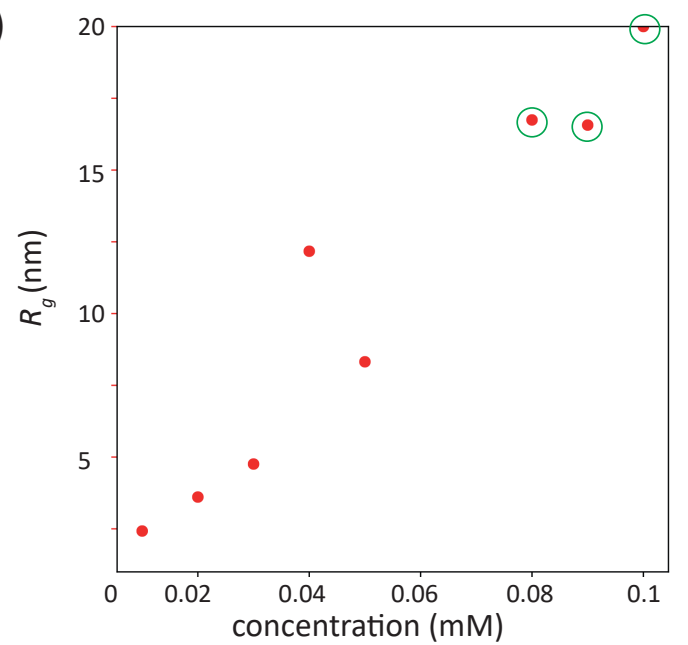

Figure 5.21: Analysis of vimentin assembled with the tetravalent molecule spermine using the model described by Pedersen. An additional term to allow tetramers in the system is added. (a) Retrieved radius of the vimentin filament at different salt concentrations show an overall increase with increasing ion concentration. Only the calculated radius at an ion concentration of $0.03 \mathrm{mM}$ is lower. (b) The calculated radius of gyration of the tails of vimentin at different concentrations. An overall increase of the radius of gyration of the filaments with increasing ion concentrations is observed. Data from precipitated vimentin is marked with green circles.

trations, which is unexpected. Going to higher spermine concentrations, similar values for the filament radius are retrieved at concentrations of $0.04 \mathrm{mM}$ and $0.05 \mathrm{mM}$. The radius of gyration $R_{g}$ of the tails increase with increasing ion concentration showing a linear trend. However, the $R_{g}$ retrieved at $0.04 \mathrm{mM}$ spermine has a large value compared to the other data. The amount of tetramers does not follow a decreasing trend as seen with mono- and divalent ions (Fig. 5.22). For the lowest two concentrations the amount of tetramers is roughly $80 \%$. When vimentin is assembled in the presence of $0.03 \mathrm{mM}$ spermine, the amount of tetramers is zero. It increases again to roughly $50 \%$ when vimentin is assembled with $0.04 \mathrm{mM}$ spermine. It decreases again to roughly $20 \%$ for assembled vimentin in the presence of $0.05 \mathrm{mM}$ spermine. Comparing the values of the filament radius retrieved from the model with the values gained by Guinier analysis, the values are slightly different but still in the same range.

Fluorescence microscopy experiments are performed for vimentin assembled with $0.03 \mathrm{mM}$ spermine and an image is shown in Fig. 5.23.

When assembling vimentin with spermine, networks can be observed. The overall network size in the image is roughly $10 \mu \mathrm{m}$ in diameter. Comparing it to the trivalent ion it has a similar network size but both network sizes are smaller than what is observed with divalent ions. 


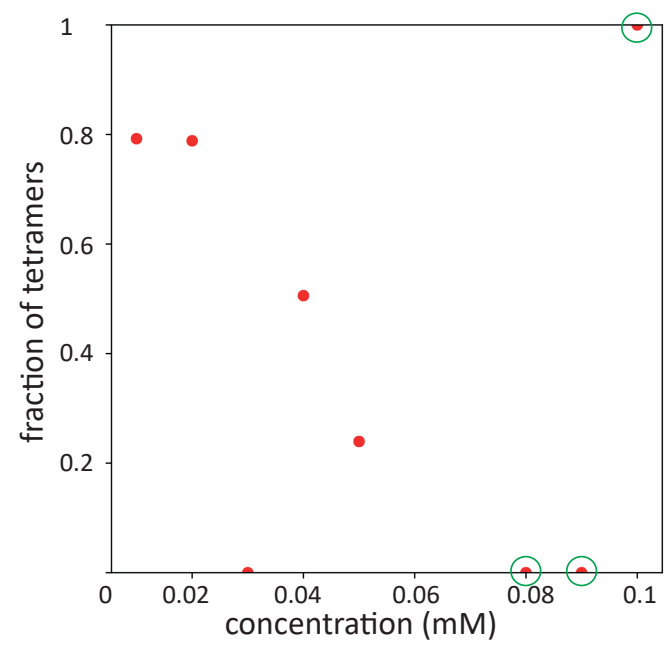

Figure 5.22: The calculated fraction of tetramers in the measured solution for vimentin protein assembled with the tetravalent molecule spermine. The amount of tetramers in the system at the two lowest ion concentrations is very similar. For $0.03 \mathrm{mM}$ spermine the amount of tetramers in the solution reduces to zero. An amount of roughly $50 \%$ tetramers in the solution is found at an spermine concentration of $0.04 \mathrm{mM}$. Data from precipitated vimentin is marked with green circles.

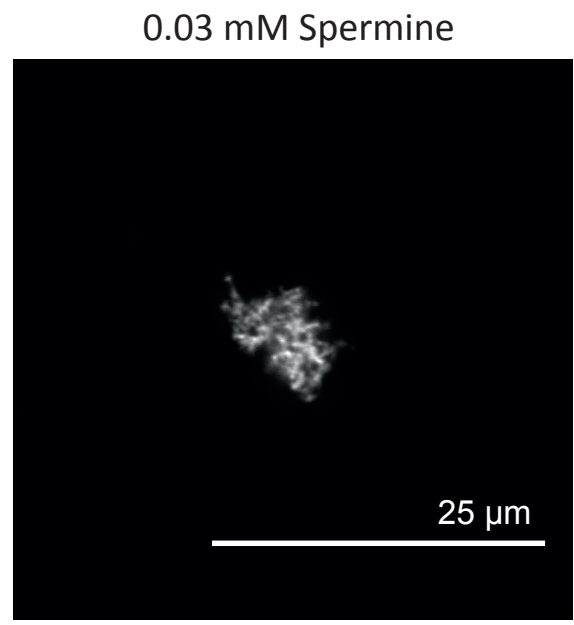

Figure 5.23: Microscopy image of vimentin assembled with $0.03 \mathrm{mM}$ spermine. Like with all ions of higher valeny, vimentin forms networks when assembled with spermine. The size of the network is smaller than what is observed with divalent ions.

\subsection{Summary and Discussion}

The influence of different ions with varying valencies and concentrations on the assembly of vimentin is investigated by SAXS and fluorescence microscopy measurements. From the SAXS data, the Guinier region ( $R_{c}$ and $I(0)$ values), as well as the mean steepness and the mean curvature are analyzed. From all three methods of analysis information about the radius of the 
filament can be retrieved. Whereas the Guinier region yields the radius of gyration of the crosssection, both the steepness and the curvature are indicators of a radius change. Especially in the case of the steepness, similar values between different measurements correspond to similar radii. Furthermore, the $I(0)$ values can be extracted from the Guinier analysis. An increase in the $I(0)$ values indicates that the molecular weight of the protein increases, which means the number of subunits per cross-section in the filament increases. The three mentioned methods to analyze the data are so-called model-free methods. In addition to the model-free data analysis, a model is applied to fit the data. The model was first introduced by Pedersen [16] and extended by an additional term to include a tetrameric contribution of vimentin protein in the solution as described by Brennich et al. [2]. From the model, the filament radius, the radius of gyration of the tails, as well as the fraction of tetramers in the solution can be calculated.

When vimentin is assembled in the presence of the monovalent salts, $\mathrm{NaCl}$ and $\mathrm{KCl}$, no significant difference between the values retrieved for the radius can be observed with the modelfree or model-based analysis. With increasing ion concentration, the radius of the filament increases. From Guinier analysis it can furthermore be observed that the number of subunits per cross-section increases with increasing ion concentration (indicated by the increase of the $I(0)$ value; Fig. 5.3b). The radius of gyrations of the tails increase as well with increasing ion concentrations. Brennich et al. performed a similar experiment with vimentin protein assembled at five different concentrations of $\mathrm{KCl}$ and fitting the data to the same model described here [2]. Comparing the radii Brennich et al. calculated with the radii retrieved here, similar values are observed. For the radii of gyration $\left(R_{g}\right)$ of the tails, Brennich et al. found a decrease of the values for $\mathrm{KCl}$ concentrations above $50 \mathrm{mM}$, which could not be observed in our analysis. A reason for the difference could be that Brennich et al. modeled the radius of the filament as a Gaussian distribution. As expected in both cases, the fraction of tetramers reduces with increasing ion concentrations, as with higher ion concentrations more vimentin should be assembled. In addition to the SAXS experiments, fluorescence microscopy experiments are performed and vimentin filaments in the presence of $100 \mathrm{mM} \mathrm{KCl} / \mathrm{NaCl}$ are investigated and single filaments are observed, which is in agreement with the literature $[3,19,20]$.

When using the divalent ions $\mathrm{Ca}^{2+}$ and $\mathrm{Mg}^{2+}$ to assemble vimentin tetramers into filaments in a range of $0.5 \mathrm{mM}$ to $10 \mathrm{mM}$ ion concentration, precipitation of vimentin filaments can be observed over a threshold ion concentration. The precipitation threshold for vimentin assembled with $\mathrm{CaCl}_{2}(2.0 \mathrm{mM}-2.5 \mathrm{mM})$ is lower than the threshold in presence of $\mathrm{MgCl}_{2}(4 \mathrm{mM}-5 \mathrm{mM})$. This already indicates that there is a difference between vimentin assembled with different divalent ions. Again, in the model-free and model-based analysis it can be observed that the radius of the filament increases with increasing ion concentrations. The number of subunits per crosssection (retrieved from the $I(0)$ values from the Guinier analysis) increases with increasing ion 
concentration. Comparing vimentin at the highest ion concentration before precipitation of the protein, the retrieved radii, the number of subunits as well as the amount of tetramers in the solution is similar. Brennich et al. performed static SAXS experiments not only on vimentin assembled with $\mathrm{KCl}$ but also using $\mathrm{MgCl}_{2}$ [2]. When comparing the values of the radii, the values from Brennich et al. are in agreement with our results. The calculated radius of gyration of the tails $\left(R_{g}\right)$ show an increase for vimentin assembled with increasing $\mathrm{CaCl}_{2}$ concentrations. Notably, for assembly with $\mathrm{MgCl}_{2}$ the values for ion concentrations of $1.0 \mathrm{mM}$ and $1.5 \mathrm{mM}$ are higher than for $2.0 \mathrm{mM}$. This trend is not observed in the data published by Brennich et al. [2]. A reason for the different observations could again be the difference in modeling the radius of the filament differently.

In the microscopy images, no single filaments but vimentin networks can be observed when vimentin protein is assembled with $2 \mathrm{mM} \mathrm{CaCl}$ or $4 \mathrm{mM} \mathrm{MgCl}_{2}$ (Fig. 5.13). As $\mathrm{MgCl}_{2}$ as well as $\mathrm{CaCl}_{2}$ can cross-link filaments, vimentin filaments collapse to networks when the ion concentration exceeds a certain threshold $[2,14]$. From microscopy images the network size for vimentin assembled with $\mathrm{MgCl}_{2}$ is larger than for filaments assembled with $\mathrm{CaCl}_{2}$. However, the networks found when assembling with $\mathrm{CaCl}_{2}$ are fewer, indicating that there might be a larger network, which was not observed during the experiments. Another reason could be that the network itself is more densely packed than the vimentin network assembled with $\mathrm{MgCl}_{2}$. Comparing the found results with the literature, similarities as well as differences are observed. Hofmann et al. performed viscosity and microscopy measurements of vimentin assembled with $\mathrm{NaCl}, \mathrm{KCl}, \mathrm{MgCl}_{2}$ and $\mathrm{CaCl}_{2}$, where they found no differences between vimentin assembled with $160 \mathrm{mM} \mathrm{NaCl}$ or $\mathrm{KCl}$ and vimentin assembled with $5 \mathrm{mM} \mathrm{MgCl}_{2}$. In our case, assembly with $5 \mathrm{mM} \mathrm{MgCl}_{2}$ leads to gelation (densely packed and insoluble filament/networks) [21, 22]. In addition, Hofmann et al. found filaments, assembled with $\mathrm{CaCl}_{2}$ at concentrations of $1 \mathrm{mM}$ and $2 \mathrm{mM}$, which are indistinguishable to filaments assembled with $\mathrm{NaCl}$. However, filaments were inhomogeneous with diameters up to $20 \mathrm{~nm}$, when vimentin is assembled with $5 \mathrm{mM} \mathrm{CaCl}_{2}$. In our case, a network-like structure for vimentin assembled in the presence of $2 \mathrm{mM} \mathrm{CaCl}_{2}$ and a filament diameter of roughly $20 \mathrm{~nm}$ is found. At higher ion concentrations, the vimentin samples could not be investigated by microscopy as gelation of vimentin filaments occurred. Thus, similar results but at different concentrations could be observed comparing the results from Hofmann et al. with our findings. This is likely caused by the bovine instead of human origin of the vimentin protein used in Hofmann's study. Furthermore, the bovine vimentin used by Hofmann et al. was not purified from E. coli but extracted from bovine lens tissue. Studies showed that vimentin purified from E. coli is more efficient than vimentin extracted from tissue [10]. In a different study, Herrmann et al. assembled vimentin proteins in the presence of $2.5 \mathrm{mM} \mathrm{MgCl}$ and found filaments of varying diameter [12]. Mass-per-length measurements revealed that fil- 
aments assembled with $2.5 \mathrm{mM} \mathrm{MgCl}_{2}$ have two to three times more subunits per cross-section than a filament assembled with monovalent ions. In our data, the $I(0)$ value, for vimentin assembled with $100 \mathrm{mM} \mathrm{KCl}$ is 0.054 , whereas for vimentin assembled with $2.5 \mathrm{mM} \mathrm{MgCl}_{2}, I(0)=$ 0.103 . The $I(0)$ value retrieved for vimentin assembled with $\mathrm{MgCl}_{2}$ is nearly twice as large, which implies that there are on average approximately twice as many subunits in vimentin filaments assembled with $\mathrm{MgCl}_{2}$ compared to filaments assembled in the presence of $\mathrm{KCl}$.

When assembling vimentin with the trivalent salt hexammine-cobalt(III) chloride, the filaments start to precipitate between an ion concentration of $0.1 \mathrm{mM}$ and $0.2 \mathrm{mM}$. This is roughly a magnitude lower than what is found with the divalent ions $\mathrm{CaCl}_{2}$ and $\mathrm{MgCl}_{2}$. At an ion concentration lower than $0.2 \mathrm{mM}$, the radius of the vimentin filaments, as well as the number of subunits increases with increasing ion concentration. When applying the micelle model to the vimentin data, an overall increase of the filament radius with increasing ion concentration is observed (Fig. 5.16). For the radius of gyration of the tails $R_{g}$ an increase can be observed, however at the lowest three concentrations $(0.01 \mathrm{mM}, 0.02 \mathrm{mM}$ and $0.03 \mathrm{mM})$ the values retrieved have no increasing trend with increasing ion concentration (Fig. 5.16). This is also observed in the filament radius. Looking at the scattering profiles of vimentin assembled with $0.01 \mathrm{mM}$ or $0.02 \mathrm{mM}$ hexammine-cobalt(III) chloride, the scattering profiles are very similar, thus the values retrieved are expected to be similar as well. However, comparison of the fraction of tetramers in the solution, a higher amount is found for vimentin assembled with $0.02 \mathrm{mM}$ hexamminecobalt(III) chloride. This indicates that there is more than one optimal solution to fit the data or the model might not be optimal to fit the data at high concentrations of multivalent ions which will be discussed later in the section. In the fluorescence microscopy image, vimentin networks can be observed, meaning that also trivalent ions have the potential to cross-link filaments. The resulting networks are smaller and and also fewer than vimentin networks assembled with divalent ions. As mentioned before, a reason could be that there is a very large network which was not observed with the microscope or the network is more dense.

Lastly, the influence of the tetravalent spermine is investigated. Again all radii (Guinier analysis and model-bases analysis) as well as the $I(0)$ values increase with increasing spermine concentration (Fig. 5.20 and Fig. 5.21). The highest measured spermine concentration at which vimentin did not precipitate is $0.05 \mathrm{mM}$. At this concentration, vimentin has a radius of roughly $8 \mathrm{~nm}$. Regarding the results from the model fits, again, an overall increase of the radius, as well as the radius of gyration of the tails with increasing ion concentration can be observed. The radius of the filament found from the model fit for vimentin filaments assembled in the presence of $0.03 \mathrm{mM}$ spermine is smaller than at lower ion concentrations, which is unexpected, however, the micelle model further yields a tetrameric concentration of zero, which is also remarkable. The fit to the data looks good, indicating that there might be more than one optimal solution, 
or the model fails to describe the data properly as discussed later. Microscopy images show that networks are formed when vimentin is assembled in the presence the tetravalent molecule spermine. The networks are, again, fewer and smaller compared to vimentin filaments assembled with divalent ions and similar in size observed for vimentin filaments assembled in the presence of the trivalent ion hexammine-cobalt(III) chloride.

As mentioned above, the micelle model with the additional term to include tetramers in the solution fails to describe the vimentin filaments accurately at high concentrations of multivalent ions. The model used to fit the data is based on a cylinder and, furthermore, the model assumes that there are 32 tails protruding from the filament core, which corresponds to 32 subunits per cross-section within the filament. When looking at the values retrieved from Guinier analysis it is evident that the number of subunits per cross-section is larger for vimentin filaments assembled at higher concentrations. Values corresponding to three to four times more subunits per cross-section are found for multivalent ions compared to vimentin assembled at $100 \mathrm{mM} \mathrm{KCl} / \mathrm{NaCl}$ (32 subunits per cross-section [5]). Calculation of the number of subunits per cross-section using the $I(0)$ values shows that at $2 \mathrm{mM} \mathrm{CaCl}_{2}$ vimentin has roughly 4 times more subunits than in the presence of $100 \mathrm{mM} \mathrm{KCl}$. The number of subunits per cross-section is adapted in the model to 128 and the data is fitted again (Fig. 5.24).

(a)

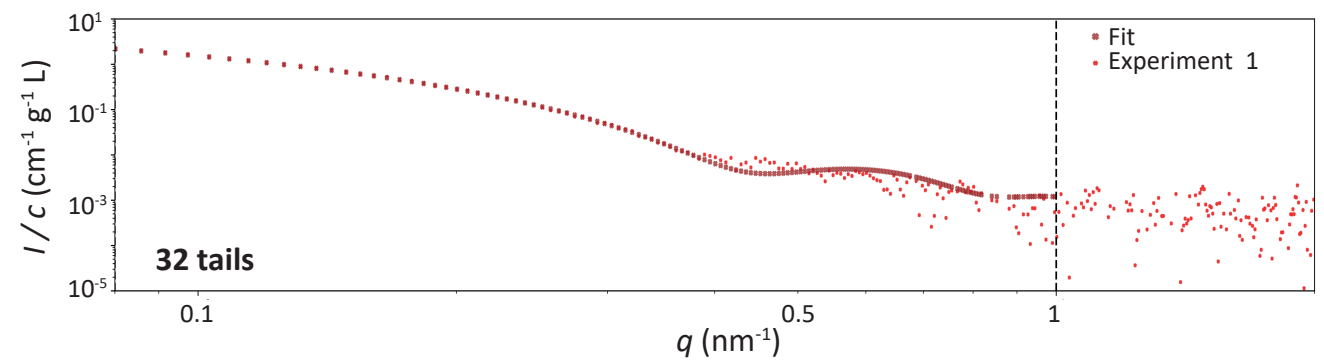

(b)

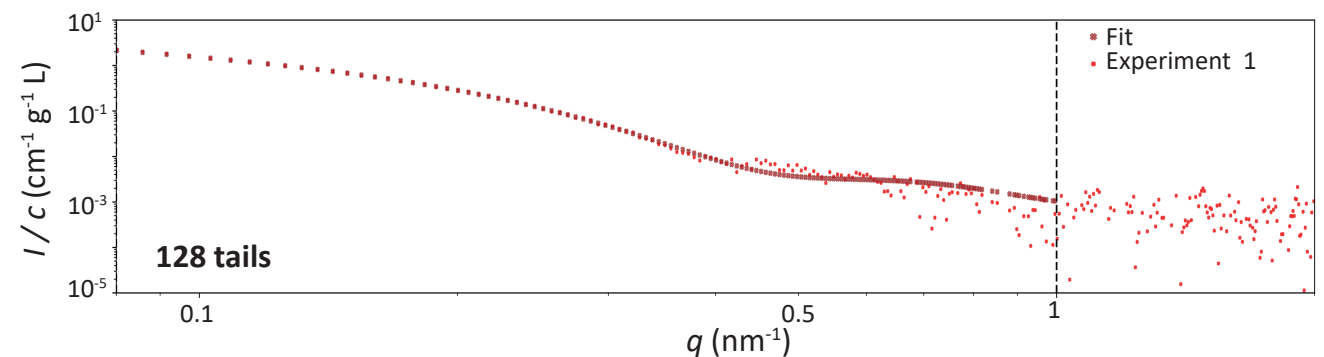

Figure 5.24: Model fit to vimentin assembled in the presence of $2 \mathrm{mM} \mathrm{CaCl}_{2}$. (a) Model fit when 32 subunits per cross-section are assumed. (b) Model fit when the number of subunits is adjusted to 128. The value is calculated based on the $I(0)$ values retrieved from the Guinier analysis.

It can be observed that the quality of the fit clearly improves and represents the data better. Thus, it is necessary to adapt the number of tails for the fit. The number of subunits per cross- 
section and thereby the number of tails can be based on the $I(0)$ values from Guinier analysis. The fit is better however, not optimal, suggesting that maybe the model itself is no longer optimal to describe the data perfectly.

Taking together all results several observations can be made. In contrast to monovalent ions, multivalent ions have the potential to cross-link filaments so that networks are formed. This phenomenon can be explained by Manning's theory of counterion condensation. In the theory, a infinitely thin rod with an homogeneous line charge is assumed, where counterion condensation takes place if:

$$
\zeta=l_{B} / l_{\text {charge }}>1
$$

with $l_{B}$ as the Bjerrum-length (the distance where $k_{B} T$ is equal to two elementary charges) and $l_{\text {charge }}$ the distance between the charges on the polyelectrolytes [23-25]. Vimentin filaments have a negative net charge and, the filaments therefore would repel each other. However, cations have the possibility to condensate on the filament itself and form a layer screening the filament and induce attraction between filaments, if Equation 5.1 is fulfilled. If a threshold is exceeded, the attractive forces are larger than the repulsive forces and filament networks are formed. The higher the valency of the ion is, the stronger the coupling between filaments is. Thus, the networks formed with tri- and tetravalent ions are likely to be more compact and consequently, appear smaller in the microscopy images. Furthermore, after reaching a certain ion concentration vimentin filaments start to precipitate. The range between the last nonprecipitated and the first precipitated vimentin sample is shown in Fig. 5.25.

With increasing valency of the ion the threshold for precipitation decreases. The decrease is roughly by one order of magnitude as suggested by Dammann in his PhD thesis, where he added either $\mathrm{MgCl}_{2}$ or hexammine-cobalt(III) chloride to already formed vimentin filaments [26]. Only vimentin filaments assembled in the presence of spermine do not show this decrease by one order of magnitude. A reason could be that spermine is a molecule and not an ion. Similar effects as with ions can be observed, however the data seems to follow a different principle. In Fig. 5.25 it is obvious, that vimentin filaments assembled with $\mathrm{Ca}^{2+}$ and $\mathrm{Mg}^{2+}$ do not have the same threshold for precipitation, even though they have the same valency. In literature, different threshold of aggregation were found for vimentin protein that was first assembled by monovalent ions and cross-linked with divalent ions subsequently [1, 14]. It was suggested that the efficiency of the ions increases with increasing atomic number, meaning a lower ion concentration is needed to fulfill the same reaction [1]. The same effect is observed for the vimentin filaments assembled with $\mathrm{Ca}^{2+}$ and $\mathrm{Mg}^{2+}$ in our study. To explain this phenomenon the Hofmeister effect can be applied, which describes salt-protein interactions [27-29]. Until today, 


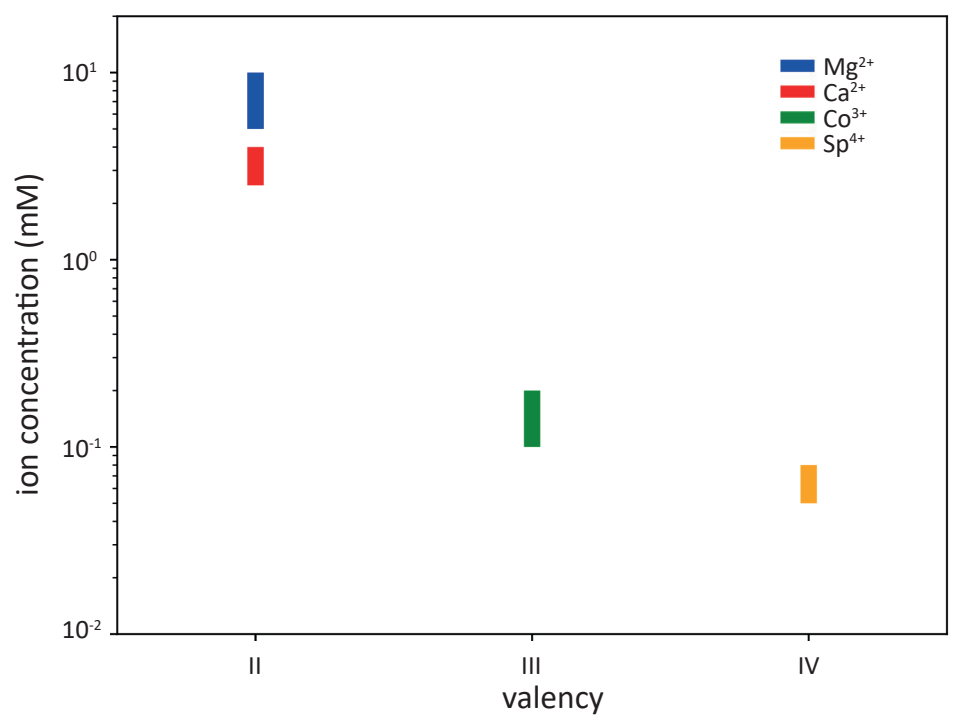

Figure 5.25: The precipitation threshold observed for vimentin assembled by different ions against the valency of the ions. With increasing valency of the ion, the threshold concentration at which vimentin filaments precipitate decreases.

the observed effects cannot be explained by electrostatic theories and the reason why the ions react differently is not known [14, 28]. The Hofmeister series is a sequence of ions (anions and cations) which orders the ions according to the concentration needed to achieve the same reaction. For cations the following order could be empirically retrieved $[28,30]$ :

$$
\left(\mathrm{CH}_{3}\right)_{4} \mathrm{~N}^{+}>\mathrm{Rb}^{+}>\mathrm{K}^{+}>\mathrm{Na}^{+}>\mathrm{Li}^{+}>\mathrm{Mg}^{2+}>\mathrm{Ca}^{2+}
$$

The ions on the right side of the sequence are more chaotropic, meaning they introduce more disorder in the system. A lower concentration is necessary for the same reaction as with ions on the left side of the series. No large differences between vimentin assembled with $\mathrm{Na}^{+}$or $\mathrm{K}^{+}$ ions is observed in the experiments shown, however, fluorescence microscopy experiments at $150 \mathrm{mM} \mathrm{NaCl}$ showed slight gelation of vimentin, while vimentin assembled with $\mathrm{KCl}$ was still soluble. However, the observed effect is not very pronounced. A stronger effect could be observed between the divalent ions investigated in this study $\left(\mathrm{Mg}^{2+}\right.$ and $\left.\mathrm{Ca}^{2+}\right)$. The lower threshold for precipitation and the stronger effect on the assembly of vimentin at the same ion concentration agrees well with the Hofmeister series.

To investigate if the threshold for precipitation can be correlated to the ionic strength, the ionic strength of the salts used in water are calculated with the following equation [31, 32]:

$$
I=\frac{1}{2} \sum_{i} c_{i} Z_{i}^{2}
$$


In the equation, $c_{i}$ refers to the concentration of the ion and $Z_{i}$ to the charge. The radius of the vimentin filament (in the example shown here, the radius of gyration of the cross-section from Guinier analysis) is plotted against the calculated ionic strength and shown in Fig. 5.26.

(a)

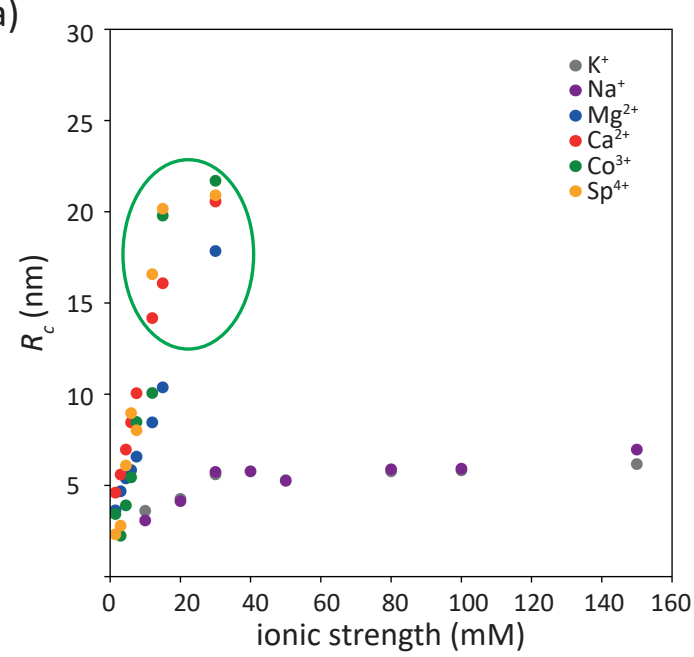

(b)

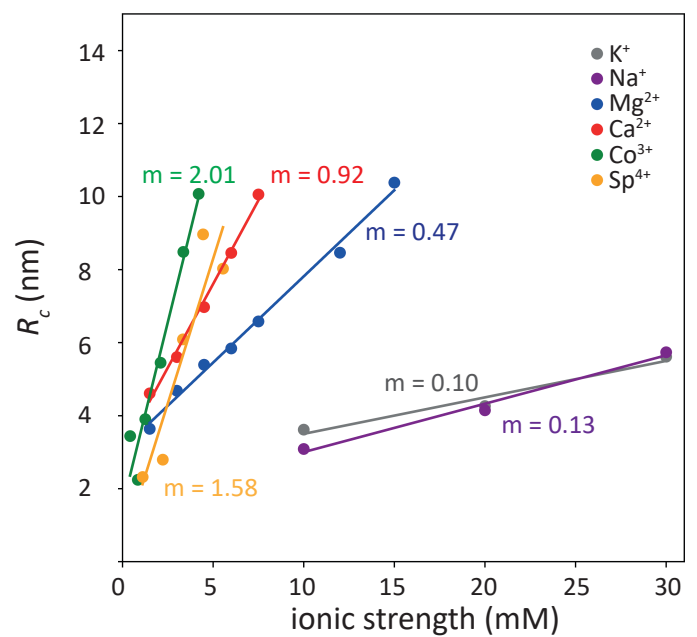

Figure 5.26: Radius of gyration of the cross-section calculated from the Guinier region in dependence of the ionic strength. (a) The radius of gyration of the cross-section for vimentin assembled by the different ions. For monovalent ions, a saturation after an ionic strength of $30 \mathrm{mM}$ can be observed. $R_{c}$ of vimentin assembled with ions of a higher valency increases until filaments start to precipitate. Precipitated protein is marked by a green circle. (b) Enlargement of (a) until an ionic strength of $30 \mathrm{mM}$. The increase in the radius seems linear, a linear fit $(y=m x+n)$ is performed and the calculated slopes are displayed.

There is no threshold of the ionic strength at which vimentin starts to precipitate. This is in agreement with Stromer et al., who proposed that not only the ionic strength is changed with different ions added to the buffer but also the interaction of the ion with the protein changes [13]. The radius of vimentin filaments assembled by monovalent ions reaches a plateau over an ionic strength of roughly $30 \mathrm{mM}$. For vimentin filaments assembled by ions of higher valencies no such plateau is observed, as filaments started to precipitate. The radii of the filaments for vimentin protein before precipitation show a linear increase with respect to the ionic strength and the data is fit with $y=m x+n$ (Fig. 5.26b). Data for vimentin assembled in the presence of monovalent ions which are not in the plateau region (up to an ionic strength of $30 \mathrm{mM}$ ) are included. It can be observed that the linear fit describes the data well. One exception is vimentin assembled in the presence of spermine which differs from the linear fit. From all fits, the slope is retrieved and it can be observed that vimentin filaments assembled by both monovalent ions have similar slopes. In contrast, the slopes at the two divalent ions differ. The slope for vimentin assembled in the presence of $\mathrm{MgCl}_{2}$ is roughly $0.5 \mathrm{~nm} / \mathrm{mM}$ whereas the slope extracted for $\mathrm{CaCl}_{2}$ is around $0.9 \mathrm{~nm} / \mathrm{mM}$. For vimentin assembled with $\mathrm{Co}^{3+}$ ions the slope is around $2 \mathrm{~nm} / \mathrm{mM}$. Thus, it can be observed that the slopes follow the order $\mathrm{K}=\mathrm{Na}<\mathrm{Mg}^{2+}<$ 
$\mathrm{Ca}^{2+}<\mathrm{Co}^{3+}$. First of all, this shows that for increasing valency of the ion, the slope increases. Furthermore, the order found in the slopes matches the Hofmeister series. Vimentin protein assembled with $\mathrm{Co}^{3+}$ has a larger slope than vimentin protein assembled in the presence of $\mathrm{Ca}^{2+}$ thus, the Hofmeister series could be extend by $\mathrm{Co}^{3+}$. Note that the fits do not intersect the $R_{c}$ axis at the same position as expected, as at an ionic strength of zero, the radius of vimentin filaments should be the same in all measurements. A reason is that a minimal ion concentration is needed to initiate vimentin assembly. Below this concentration no effects on the radius of the protein will be observed. Thus, the linear fits cannot be extrapolated linearly to an ionic strength of zero. In addition to the slopes, it can be observed that there might be an upper limit of the filament radius before vimentin starts to precipitate. For all ions, filaments of up to $10 \mathrm{~nm}$ radius can be observed. Above that, vimentin precipitates regardless of the ion used for assembly. It seems that after a certain filament diameter (roughly $10 \mathrm{~nm}$ ), the protein collapses and bundles or aggregates to very dense networks (precipitation). Plotting the $I(0)$ values against the radius of the filament (exemplary the radius of gyration of the cross-section from Guinier analysis is taken), it can be observed that there is no linear relation between the radius of the filament and the $I(0)$ values which are related to the number of subunits per cross-section in the filament (Fig. 5.27).

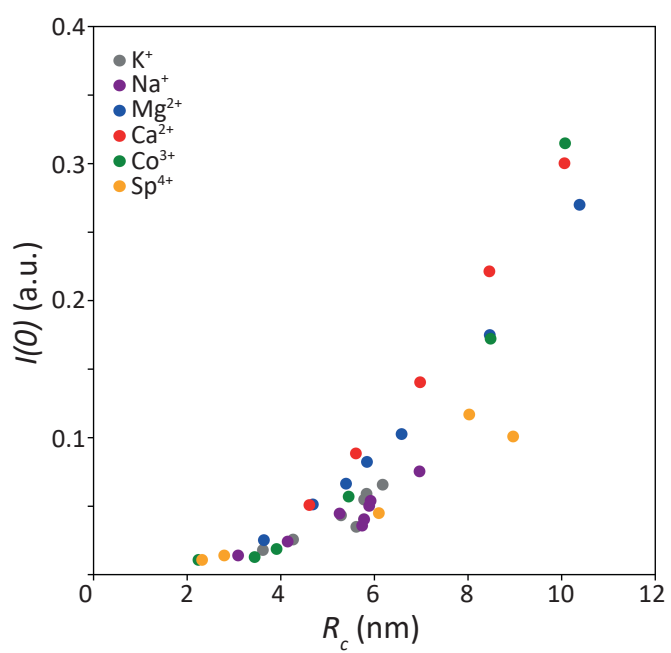

Figure 5.27: I(0) values against the radius of gyration from Guinier analysis. Plotting the I(0) values against the radius of gyration of the cross-section it can be observed that with increasing radius the I(0) values increase as well.

This indicates that with increasing radius more subunits per cross-section are incorporated in the filament. It seems that at smaller radii, the number of subunits is similar regardless of the ion used for assembly. Again, the data taken for vimentin filaments assembled in the presence of spermine does not fit into the general trend observed. Looking at the number of subunits 
per cross-section in the filaments with a radius of $10 \mathrm{~nm}$, it can be observed that the values are much higher than what is found for vimentin filaments assembled with $100 \mathrm{mM} \mathrm{KCl} / \mathrm{NaCl}$. More than 150 subunits are found when vimentin filaments are assembled in the presence of $\mathrm{Mg}^{2+}, \mathrm{Ca}^{2+}$ or $\mathrm{Co}^{3+}$ (based on the assumption that 32 subunits are present when vimentin is assembled with $100 \mathrm{mM}$ monovalent ions).

All in all, ions of different valencies strongly affect the assembly of vimentin intermediate filaments. In the presence of monovalent ions single filaments are formed with a radius of roughly $6 \mathrm{~nm}$ which are not cross-linked or precipitated. In contrast, all multivalent ions investigated have the ability to cross-link filaments. Different thresholds for precipitation (aggregation to very dense networks) of vimentin filaments are found. The higher the valency of the salt is, the lower the threshold for precipitation is. The difference between the divalent ions can be described by the Hofmeister effect. Furthermore, it seems that the filament reaches a radius of roughly $10 \mathrm{~nm}$ before the protein starts to precipitate. 


\section{References}

1. Janmey, P. A., Slochower, D. R., Wang, Y.-H., Wen, Q. \& Cēbers, A. Polyelectrolyte properties of filamentous biopolymers and their consequences in biological fluids. Soft Matter 10, 1439 (2014).

2. Brennich, M. E. et al. Impact of ion valency on the assembly of vimentin studied by quantitative small angle X-ray scattering. Soft Matter 10, 2059-2068 (2014).

3. Block, J. et al. Nonlinear Loading-Rate-Dependent Force Response of Individual Vimentin Intermediate Filaments to Applied Strain. Phys. Rev. Lett. 118, 048101 (2017).

4. Dammann, C., Nöding, B. \& Köster, S. Vimentin networks at tunable ion-concentration in microfluidic drops. Biomicrofluidics 6, 022009 (2012).

5. Dammann, C., Herrmann, H. \& Köster, S. Competitive Counterion Binding Regulates the Aggregation Onset of Vimentin Intermediate Filaments. Isr. J. Chem. 56, 614-621 (2015).

6. Block, J., Schroeder, V., Pawelzyk, P., Willenbacher, N. \& Köster, S. Physical properties of cytoplasmic intermediate filaments. Biochim. Biophys. Acta, Mol. Cell Res. 1853, 30533064 (2015).

7. Yao, N. Y. et al. Elasticity in Ionically Cross-Linked Neurofilament Networks. Biophys. J. 98, 2147-2153 (2010).

8. Tang, J. X., Wong, S., Tran, P. T. \& Janmey, P. A. Counterion induced bundle formation of rodlike polyelectrolytes. Berichte der Bunsengesellschaft für physikalische Chemie 100, 796806 (1996).

9. Tang, J. X. \& Janmey, P. A. The Polyelectrolyte Nature of F-actin and the Mechanism of Actin Bundle Formation. J. Biol. Chem. 271, 8556-8563 (1996).

10. Hofmann, I., Herrmann, H. \& Franke, W. W. Assembly and structure of calcium-induced thick vimentin filaments. Eur. J. Cell Biol. 56, 328-341 (1991).

11. Kooijman, M., Bloemendal, M., Traub, P., van Grondelle, R. \& van Amerongen, H. Transient Electric Birefringence Study of Intermediate Filament Formation from Vimentin and Glial Fibrillary Acidic Protein. J. Biol. Chem. 272, 22548-22555 (1997).

12. Herrmann, H., Häner, M., Brettel, M., Ku, N.-O. \& Aebi, U. Characterization of distinct early assembly units of different intermediate filament proteins. J. Mol. Biol. 286, 1403-1420 (1999).

13. Stromer, M. H., Ritter, M. A., Pang, Y. Y. \& Robson, R. M. Effect of cations and temperature on kinetics of desmin assembly. Biochem. J. 246, 75-81 (1987). 
14. Wu, H. et al. Effect of the divalent cations zinc and calcium on the structure and mechanics of reconstituted vimentin intermediate filaments (2019).

15. Schepers, A. V., Lorenz, C. \& Köster, S. Tuning intermediate filament mechanics by indirect and direct charge variations (2019).

16. Pedersen, J. S. Form factors of block copolymer micelles with spherical, ellipsoidal and cylindrical cores. J. Appl. Crystallogr. 33, 637-640 (2000).

17. Hémonnot, C. Y. J., Mauermann, M., Herrmann, H. \& Köster, S. Assembly of Simple Epithelial Keratin Filaments: Deciphering the Ion Dependence in Filament Organization. Biomacromolecules 16, 3313-3321 (2015).

18. Chaigne-Delalande, B. \& Lenardo, M. J. Divalent cation signaling in immune cells. Trends Immunol. 35, 332-344 (2014).

19. Kreplak, L. \& Fudge, D. Biomechanical properties of intermediate filaments: from tissues to single filaments and back. Bioessays 29, 26-35 (2006).

20. Steinert, P. M., Idler, W. W. \& Zimmerman, S. B. Self-assembly of bovine epidermal keratin filaments in vitro. J. Mol. Biol. 108, 547-567 (1976).

21. Huisman, E. M. et al. Gelation of semiflexible polyelectrolytes by multivalent counterions. Soft Matter 7, 7257 (2011).

22. Charrier, E. E. \& Janmey, P. A. in Methods in Enzymology 35-57 (Elsevier, 2016).

23. Manning, G. S. Counterion binding in polyelectrolyte theory. Acc. Chem. Res. 12, 443-449 (1979).

24. Stigter, D. Evaluation of the counterion condensation theory of polyelectrolytes. Biophys. J. 69, 380-388 (1995).

25. Naghizadeh, J. Counterion condensation in polyelectrolyte theory. Cell Biophys. 11, 103108 (1987).

26. Dammann, C. Organization of the Cytoskeleton: Studies in Microfluidic Drops PhD thesis (Institute for X-Ray Physics, Georg-August-Universität Göttingen, 2014).

27. Hofmeister, F. Zur Lehre von der Wirkung der Salze. Arch. Exp. Pathol. Pharmakol. 24, 247260 (1888).

28. Salis, A. \& Ninham, B. W. Models and mechanisms of Hofmeister effects in electrolyte solutions, and colloid and protein systems revisited. Chem. Soc. Rev. 43, 7358-7377 (2014).

29. Baldwin, R. How Hofmeister ion interactions affect protein stability. Biophys. J. 71, 20562063 (1996). 
30. Okur, H. I. et al. Beyond the Hofmeister Series: Ion-Specific Effects on Proteins and Their Biological Functions. J. Phys. Chem. B 121, 1997-2014 (2017).

31. Arnaut, L., Formosinho, S. \& Burrows, H. in Chemical Kinetics 223-250 (Elsevier, 2007).

32. Solomon, T. The Definition and Unit of Ionic Strength. J. Chem. Educ. 78, 1691 (2001). 


\section{Chapter 6}

\section{Cyclic olefin copolymer as an X-ray compatible material for microfluidic devices}

The results presented in this chapter are published as an article in Lab on a Chip. Reproduced from Ref. Cyclic olefin copolymer as an X-ray compatible material for microfluidic devices, M. Denz and G. Brehm et al., Lab Chip, 18, 171-178 (2018). DOI: 10.1039/C7LC00824D with permission from The Royal Society of Chemistry.

Manuela Denz, $\ddagger^{a}$ Gerrit Brehm, $\ddagger^{a b}$ Clément Y. J. Hémonnot, ${ }^{a}$ Heidi Spears, ${ }^{a}$ Andrew Wittmeier, ${ }^{a}$ Chiara Cassini, ${ }^{a}$ Oliva Saldanha, ${ }^{a}$ Eleonora Perego, ${ }^{a}$ Ana Diaz, ${ }^{c}$ Manfred Burghammer $^{d}$ and Sarah Köster*ab

${ }^{a}$ Institute for X-Ray Physics, University of Goettingen, 37077 Göttingen, Germany.

E-mail: sarah.koester@phys.uni-goettingen.de

${ }^{b}$ Center Nanoscale Microscopy and Molecular Physiology of the Brain (CNMPB),Göttingen, Germany

${ }^{c}$ Swiss Light Source, Paul Scherrer Institut, 5232 Villigen, Switzerland.

${ }^{d}$ European Synchrotron Radiation Facility, 38043 Grenoble, France.

$\ddagger$ These authors contributed equally to this work.

Contribution to the article: Susanne Bauch produced and isolated the vimentin wt protein and prepared the buffers for vimentin experiments. I have prepared the vimentin and the buffers for the colloid experiments. I have adapted the protocol for UV-curable adhesive/Kapton devices 
and produced most of the UV-curable adhesive/Kapton devices. Gerrit Brehm produced the COC devices and adapted the protocol for it. Clément Y. J. Hémonnot and Heidi Spears made the first version of the COC protocol. Clément Y. J. Hémonnot implemented the Mathlab script for azimuthal integration based on a script by Oliver Bunk (Paul Scherrer Institute, Villigen, Switzerland). Andrew Wittmeier, Chiara Cassini, Oliva Saldanha and Eleonora Perego helped conducting the experiments at the synchrotron. Ana Diaz (cSAXS) and Manfred Burghammer (ID13) helped at the beamline. I have performed the data analysis. I have made the Figures 1, 2, 4, 5 and S1. The first version of the manuscript has been written by Gerrit Brehm and myself. It was then iteratively optimized by Gerrit Brehm, Sarah Köster and myself. The co-authors have commented the paper right before submission.

\subsection{Abstract}

The combination of microfluidics and X-ray methods attracts a lot of attention from researchers as it brings together the high controllability of microfluidic sample environments and the small length scales probed by X-rays. In particular, the fields of biophysics and biology have benefited enormously from such approaches. We introduce a straightforward fabrication method for Xray compatible microfluidic devices made solely from cyclic olefin copolymers. We benchmark the performance of the devices against other devices including more commonly used Kapton windows and obtain data of equal quality using small angle X-ray scattering. An advantage of the devices presented here is that no gluing between interfaces is necessary, rendering the production very reliable. As a biophysical application, we investigate the early time points of the assembly of vimentin intermediate filament proteins into higher-order structures. This weakly scattering protein system leads to high quality data in the new devices, thus opening up the way for numerous future applications.

\subsection{Introduction}

Many important biological processes take place on nanometer and micrometer length scales, and microfluidics has emerged as an extremely successful field for studying such processes. For microscopy applications, microfluidics is nowadays very mature and established, especially when employing polydimethylsiloxane (PDMS) and glass as the main materials [1]. However, many processes on the molecular scale cannot be probed by light microscopy and therefore, more recently, several groups have started to combine microfluidics with small angle X-ray scattering (SAXS), which is sensitive to length scales of about 10 to $100 \mathrm{~nm}[2,3]$. To name a few ex- 
amples, researchers have investigated protein [4-13] and RNA [14-18] folding, DNA compaction $[19,20]$, and self-assembly of filaments and fibrils [21-24]. These examples clearly demonstrate the benefits of combining the high spatial resolution in X-ray techniques with the precise, controllable sample environment inside microfluidic devices, such as concentration gradients, specific flow fields, or diffusive mixing in laminar flow. As a result, time resolved investigations of reactions and mechanisms, which cannot be captured by light microscopy, due to the relevant length scales of the system, can be performed.

The success of these applications is further highlighted by recent and planned developments concerning synchrotron light sources. Highly focused and brilliant beams are well compatible with small channel dimensions and time-resolved measurements. However, as recently pointed out by Ghazal et al. [2], there is still a need for versatile, reproducible X-ray compatible microfluidic devices, which may be fabricated and used by a large community of scientists beyond the immediate group of specialists. The most important requirements for the employed materials are moldability, X-ray resistance, transparency to X-rays and a low background scattering signal. To achieve these characteristics, many design approaches have utilized combinations of different materials, including etched silicon with PDMS windows [14-18], PDMS and quartz glass capillaries [23, 25], PDMS and Kapton film [26, 27], stainless steel and Kapton film [13, 20, 22, 26, 28], optical adhesive [21, 23] and optical adhesive with Kapton film and silicon rich nitride windows [29]. Fabrication of these devices typically involves advanced engineering skills.

In X-ray crystallography, another commonly used material class are cyclic olefin copolymers (COCs). Steigert et al. introduced fabrications methods for COC devices [30] which were used for miniaturized and highly parallelized protein crystallization under well defined conditions [31]. Similar approaches, including screening of crystal growth and data collection inside the device, have been reported in the following years [32-35]. These devices used a combination of COC and PDMS to allow for the inclusion of microfluidic valves and used silanes to bond the layers. A SAXS study in a commercial all-COC device was performed on liquid crystals, which typically scatter considerably stronger than proteins [36]. Silane based binding was found to be insufficient between two COC layers [37] and thus, instead, a thin layer of COC was plasticized by solvent before bonding to the second layer [37,38]. Alternatively, thermal sealing of layers with different glass transition temperatures $T_{G}$ was reported [30, 39]. Importantly, when using heat and pressure for sealing, the thickness of the finished device depends on the applied temperature and pressure due to changes in viscosity of the COC with temperature [37].

COCs are well suited for X-ray applications due to the low photon absorption at relevant photon energies between 2 and $20 \mathrm{keV}$, as compared to PDMS and $\mathrm{SiO}_{2}$ [33]. Furthermore, COCs are optically transparent and provide resistance against many solvents [40].

Here, we adapt COCs for SAXS measurements on weakly scattering protein and present the 
reproducible fabrication and thorough characterization of an easy-to-manufacture, leak-tight, low scattering and customizable microfluidic device. As the device is made solely out of one type of COC, with similar glass transition temperature it displays no interfaces whatsoever and we circumvent additional plasticization steps. The manufacturing process is based on standard soft lithography and well established molding techniques, combined with hot embossing. Lateral channel structures can flexibly be adapted, due to the lithographic approach. We characterize the devices using gold nanoparticles and, thereafter, demonstrate the utility by recording protein SAXS data.

\subsection{Materials and Methods}

\subsubsection{Device Fabrication and Usage}

Channel structures were produced using standard soft lithography methods [1, 41]. Briefly, SU82150 negative photo resist (MicroChem, Newton, USA) was spin-coated onto a 2-inch silicon wafer (Si-wafer; MicroChemicals, Ulm, Germany) to a final height of $160 \mu \mathrm{m}$. Edge bead removal was performed before the soft bake step to achieve an ideal contact between the photo mask (Selba S.A., Versoix, Switzerland) and the substrate. The resist was exposed to UV light (365 nm) using a mask-aligner (MJB4 Mask-Aligner, Süss MicroTec AG, Garching, Germany) through the mask and the structure was developed after a post exposure step (see Fig. 6.1a). Next, we produced a PDMS (Dow Corning, Midland, USA) copy of the channel structure (see Fig. 6.1b).

The PDMS copy was then used as a stamp to obtain a copy of our channel structure made from UV-curable adhesive (NOA 81, Norland Optical Adhesives, Cranbury, NJ, USA) on a glass slide as support (see Fig. 6.1c). The PDMS was pushed into the UV-curable adhesive, which was then cured under a UV-Lamp (366 nm, NU-8 KL. Benda, Wiesloch, Germany) for 3 minutes. Subsequently, the PDMS stamp may be easily removed from the glass slide, leaving behind a channel structure in UV-curable adhesive. Excess adhesive was cut away with a scalpel. Afterwards, the structure was exposed to UV light for additional 3 minutes. Note that the obtained UV-curable adhesive stamp on the glass slide can be reused. A $240 \mu \mathrm{m}$ thick layer of COC (msc foil 029, Microfluidic ChipShop GmbH, Jena, Germany), with a glass transition temperature $T_{G}$ of $78{ }^{\circ} \mathrm{C}$, was placed on top of the channel structure on the glass slide. The ensemble was sandwiched between two sheets of paper, followed by two sheets of aluminum foil and placed in the preheated hot press (PW $10 \mathrm{H}, \mathrm{P} / \mathrm{O} /$ Weber $\mathrm{GmbH}$, Remshalden, Germany) at $130{ }^{\circ} \mathrm{C}$ for 5 minutes. The ensemble was subsequently pressed with initially $2.2 \mathrm{kN}$ for 10 minutes (see Fig. 6.1d). After cooling down, the COC structure was removed from the UV-curable adhesive structure and holes were punched for the inlets and outlet of the microfluidic device using $0.5 \mathrm{~mm}$ biopsy punch- 
a
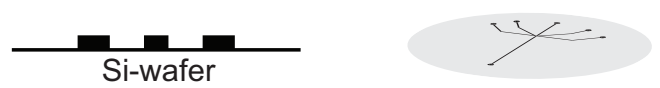

b
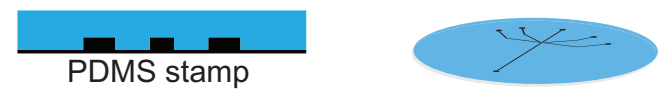

C
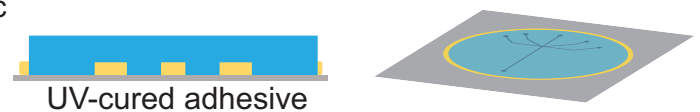

d
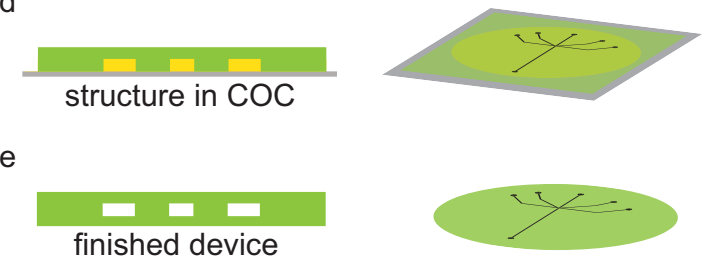

Figure 6.1: Manufacturing steps of a COC device. (a) Channel structures were fabricated by photolithography (black) using SU-8 2150 negative photo resist spin coated on Si-wafers. (b) PDMS copies (blue) of the channel structures were produced. (c) By pressing the PDMS into UV-cured adhesive (yellow) deposited on a glass slide, channel structures were replicated. (d) COC sheets (green) were heated above the glass transition temperature, and structured by hot embossing. (e) A COC sealing layer was used to close the channels.

ers (Harris Uni-CoreTM puncher, Plano, Wetzlar, Germany). Finally, the channel structure was sealed with a $20 \mu \mathrm{m}$ thick layer of COC (Topas $8007 T_{G}=75{ }^{\circ} \mathrm{C}$, TOPAS Advanced Polymers $\mathrm{GmbH}$, Frankfurt, Germany) via a heat and pressure controlled lamination machine (Catena 35 GBC, ACCO Brands, Lincolnshire, USA) at $100^{\circ} \mathrm{C}$, a speed of $7.610^{-3} \mathrm{~m} \mathrm{~s}^{-1}$ and narrowest setting for the rolls. For better handling, the device and the sealing layer were placed between two sheets of paper during sealing. The resulting microfluidic channels were measured using optical microscopy to $200 \mu \mathrm{m}$ width and $160 \mu \mathrm{m}$ height (Fig. 6.1e). For a more detailed figure with all assembly steps, see Fig. S1†. The measurement window consisted of COC with a thickness of about $50 \mu \mathrm{m}$ and additional $20 \mu \mathrm{m}$ sealing layer (see Fig. S2†). Note that during heat and pressure treatment the COC layer becomes thinner, thus accounting for the "missing" $30 \mu \mathrm{m}$ [37]. UV-curable adhesive/Kapton devices were fabricated as previously described [29]. Briefly, a PDMS copy was produced from the Si-wafer. Afterwards, a second PDMS stamp was produced from the first PDMS copy. An $8 \mu \mathrm{m}$ thick Kapton foil (SPEX SamplePrep, Metuchen, NJ, USA) with liquid adhesive (NOA 81) was prepared on an aluminum block. Adhesive was also pipetted on the PDMS structure and placed in a desiccator for 15 minutes to remove air bubbles. Subsequently, the positive PDMS structure was pressed into the adhesive on the Kapton foil to obtain the channel structure. After partially curing the adhesive, holes for the inlets and outlet were punched, using a biopsy puncher with a diameter of $0.75 \mathrm{~mm}$. The PDMS stamp was removed 
and a second Kapton foil was used to seal the device, which contained measurement windows of $16 \mu \mathrm{m}$ Kapton in total and possibly a thin layer of UV-cured adhesive. In the finished device, the channels had a width of $200 \mu \mathrm{m}$ and a height of $160 \mu \mathrm{m}$.

As a geometry for all devices, we chose a five-inlet/one-outlet system with $45^{\circ}$ angles between the inlet channels (Fig. 6.2a). Through the central inlet, protein $\left(5.5 \mathrm{mg} \mathrm{mL}^{-1}\right)$ or gold colloid solution (Sigma Aldrich, St. Louis, Missouri, USA; radius $R=15 \mathrm{~nm}$, concentration in the syringe $c=1.8 \times 10^{11}$ particles per $\mathrm{mL}$ and $R=10 \mathrm{~nm}, c=6.5 \times 10^{11}$ particles per $\mathrm{mL}$ ) was injected at a rate of $15 \mu \mathrm{L} \mathrm{h}^{-1}\left(1.3 \times 10^{-4} \mathrm{~m} \mathrm{~s}^{-1}\right)$. Sheath inlets [42], which were situated on both sides of the central inlet, were injected with buffer at a low flow rate of $7.5 \mu \mathrm{L} \mathrm{h}^{-1}\left(6.5 \times 10^{-5} \mathrm{~m} \mathrm{~s}^{-1}\right)$. The side inlets contained $110 \mathrm{mM} \mathrm{KCl}$ in buffer for protein experiments and phosphate buffered saline (PBS) for gold colloid experiments at a flow rate of $150 \mu \mathrm{L} \mathrm{h}^{-1}\left(1.3 \times 10^{-3} \mathrm{~m} \mathrm{~s}^{-1}\right)$.

Polyethylene tubing (inner diameter $0.38 \mathrm{~mm}$, outer diameter $1.09 \mathrm{~mm}$, Intramedic Clay Adams Brand, Becton Dickinson and Company, Sparks, USA) was connected to Hamilton Gastight glass syringes (Bonaduz, Switzerland) with $1 \mathrm{~mL}$ or $0.5 \mathrm{~mL}$ volume by disposable needles. Syringe pumps (neMESYS, Cetoni GmbH, Korbußen, Germany) were employed for precise flow control over all five inlets. A sample holder based on the one introduced in Urbani's dissertation [43], was used to tightly connect the tubing and device in a leak-free manner. Briefly, the sample holder consisted of two metal plates that sandwich one PVC (polyvinylchloride) plate and the device itself. The metal and PVC plates were equipped with an opening that served as a measurement window. Tubing was threaded through small holes in the front metal and the PVC plate as well as o-rings for sealing. The device was aligned with the tubing and fixed to the PVC plate with sticky tape. The whole ensemble was screwed onto the metal back plate, which was larger then the other plates and served as an adapter plate to the beamline sample stage. A photograph of the sample holder used for the experiments is shown in Fig. S3†.

\subsubsection{Protein Purification and Assembly}

Human vimentin protein with a molecular weight of $53.7 \mathrm{kDa}$ was expressed in Escherichia coli bacteria and then purified from inclusion bodies [44]. Vimentin was stored at $-80^{\circ} \mathrm{C}$ in $8 \mathrm{M}$ urea, 5 mM Tris-HCl (pH 7.5), 1 mM EDTA, 0.1 mM EGTA, 1 mM DTT and 10 mM methyl ammonium chloride (MAC). The purity of the protein was verified by SDS-polyacrylamide gel electrophoresis. All solutions were prepared using $2 \mathrm{mM}$ phosphate buffer (PB), $\mathrm{pH}$ 7.5. Before use in the experiments, the protein was dialyzed against $8 \mathrm{M}$ urea for 30 minutes and then, in a stepwise manner, against $6,4,2$, and $1 \mathrm{M}$ urea for 30 minutes each, at room temperature. Finally, an overnight dialysis into $2 \mathrm{mM} \mathrm{PB}$ was performed at $10{ }^{\circ} \mathrm{C}$. All dialysis steps were performed using membranes of $50 \mathrm{kDa}$ cut-off (SpectraPor, Carl-Roth GmbH, Karlsruhe, Germany). The pro- 


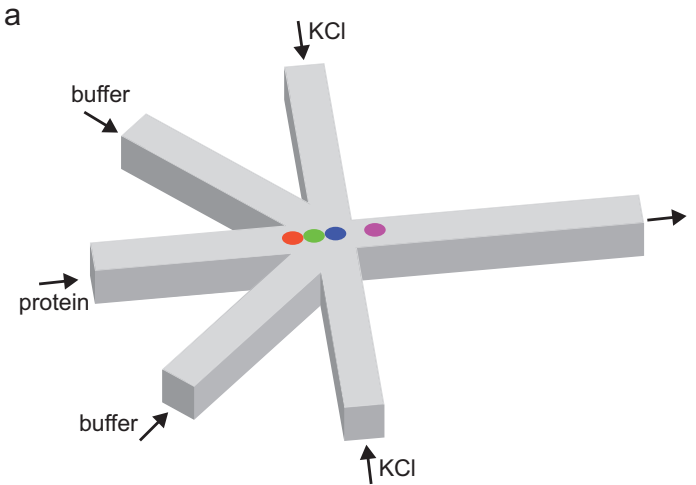

b
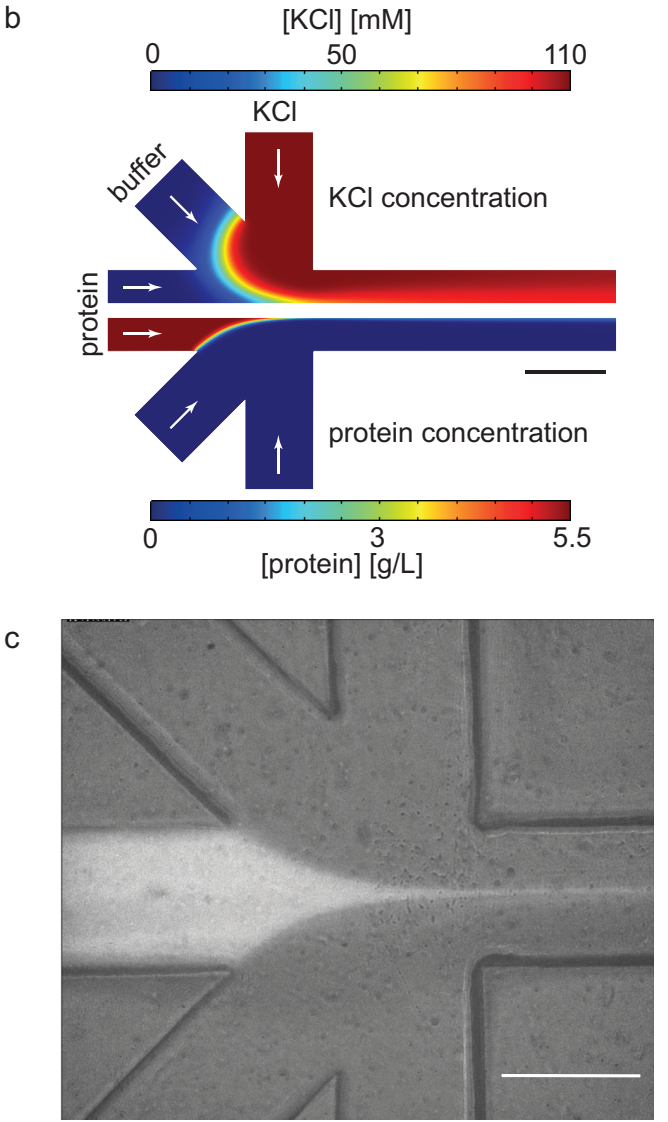

Figure 6.2: Geometry of the microfluidic device. (a) Scheme of the device layout. The sample (protein or colloid solution) was injected through the central inlet. The side inlets were used for injecting assembly start buffer with KCl for vimentin, or buffer for gold experiments. The diagonal sheath buffer inlets push the mixing point further into the outlet channel. The colored dots refer to the positions analyzed in the vimentin experiment (see Fig. 6.5). (b) Results of the FEM flow simulations: concentration of KCl (upper half of the figure) and vimentin (lower half of the figure). (c) Fluorescence and bright field micrograph (overlay) of microfluidic experiment performed with labeled vimentin (Alexa 488). All scale bars $200 \mu \mathrm{m}$.

tein concentration was determined to be $5.5 \mathrm{mg} \mathrm{mL}^{-1}$ by measuring the absorption at $280 \mathrm{~nm}$ (Nanodrop ND-1000, ThermoScientific Technologies, Inc., Wilmington, USA). Assembly was ini- 
tiated in the microfluidic devices by diffusive addition of $110 \mathrm{mM} \mathrm{KCl}$ in buffer to the vimentin solution. The calculated end concentration of $\mathrm{KCl}$ after complete mixing in the outlet channel was $100 \mathrm{mM}$, and the vimentin concentration at the last measured position shown here was $4.0 \mathrm{mg} \mathrm{mL}^{-1}$.

For microscopy experiments in a UV-curable adhesive/Kapton device, AlexaFluo 488 (Invitrogen $\mathrm{GmbH}$, Darmstadt, Germany) labeled vimentin was used [45, 46]. An inverted Olympus IX71 microscope (Olympus Europa SE \& CO. KG, Hamburg, Germany) equipped with a 10X objective was employed to image the flow. Flow rates in this experiment were $100 \mu \mathrm{L} \mathrm{h}^{-1}$ $\left(8.7 \times 10^{-4} \mathrm{~m} \mathrm{~s}^{-1}\right)$ for the side inlets $(100 \mathrm{mM} \mathrm{KCl}), 10 \mu \mathrm{L} \mathrm{h}^{-1}\left(8.7 \times 10^{-5} \mathrm{~m} \mathrm{~s}^{-1}\right)$ for the central protein inlet and $5 \mu \mathrm{L} \mathrm{h}^{-1}\left(4.3 \times 10^{-5} \mathrm{~m} \mathrm{~s}^{-1}\right)$ for the sheath inlets.

\subsubsection{Small Angle X-Ray Scattering}

SAXS measurements were performed at two different synchrotron sources, the ID13 beamline at the European Synchrotron Radiation Facility (ESRF) (Grenoble, France) and the cSAXS beamline at the Swiss Light Source (SLS) (Paul Scherer Institut, Villigen, Switzerland). All measurements were performed at room temperature and in air.

At ID13, an in-vacuum undulator was used and the energy (13.9 keV) was selected using an $\mathrm{Si}(111)$ monochromator. A transfocator in vacuum guaranteed reproducible changing of the Xray lens packages. We used beryllium compound refractive lenses composed of 54 individual lenses with parabolic shape and a radius of curvature of $200 \mu \mathrm{m}$ in the apex. Three apertures with $50 \mu \mathrm{m}, 20 \mu \mathrm{m}$ and $80 \mu \mathrm{m}$, respectively, were included in the beam path. For background reduction, a $70 \mathrm{~mm}$ long flight tube with silicon nitride entrance window and polypropylene exit window filled with helium was employed. As a beamstop, a lead cylinder with a few mm in length and about $300 \mu \mathrm{m}$ in diameter was installed. An Eiger $4 \mathrm{M}$ detector $(2070 \times 2167$ pixels, pixel size $75 \times 75 \mu \mathrm{m}^{2}$, Dectris, Baden, Switzerland) [47] was placed at $0.95 \mathrm{~m}$ from the sample. Experiments were performed at a primary beam intensity of $8 \times 10^{11} \mathrm{ph} \mathrm{s}^{-1}$ with a beam size of $2.7 \times 1.7 \mu \mathrm{m}^{2}$ and an exposure time of $1 \mathrm{~s}$.

At cSAXS, the sample was placed at a distance of $33.8 \mathrm{~m}$ from the undulator source. The beam was defined by a set of slits of $0.6 \mathrm{~mm} \times 0.4 \mathrm{~mm}$ (horizontal $\times$ vertical) at $26.0 \mathrm{~m}$ from the source. An energy of $11.2 \mathrm{keV}$ was selected with a double-crystal Si(111) monochromator placed at about $28.5 \mathrm{~m}$ from the source, which was also used for horizontal focusing. A bendable mirror at $29.4 \mathrm{~m}$ from the source further focused the beam vertically. Different sets of slits downstream of the X-ray optics were used to clean up the beam from parasitic scattering. In order to optimize the beam size along the horizontal direction, we used a pair of slits with a horizontal aperture of $0.1 \mathrm{~mm}$ placed at $12.1 \mathrm{~m}$, which helped to effectively decrease the source size but reduced the 
flux to about $50 \%$. The beam was focused at the sample position, where we measured a beam size of $12 \times 29 \mu \mathrm{m}^{2}$ with an estimated intensity of $2 \times 10^{11} \mathrm{ph} \mathrm{s}^{-1}$. The sample scattering was recorded with a Pilatus $2 \mathrm{M}$ detector $\left(1475 \times 1679\right.$ pixels, pixel size: $172 \times 172 \mu \mathrm{m}^{2}$, SLS detector group, Villigen, Switzerland) [48] at a sample-to-detector distance of $7.087 \mathrm{~m}$. An evacuated flight-tube was placed between the sample and the detector to reduce parasitic air scattering. The total exposure time was $5 \mathrm{~s}$, split up in $5 \times 1 \mathrm{~s}$.

For a pointwise background subtraction, we performed one measurement on a buffer-filled device and directly afterwards, in the same device, the actual measurement, including the sample. By this approach, we obtained a background measurement in every position of the device, thus accounting for variations between devices and between positions in the same device.

\subsubsection{Data Analysis}

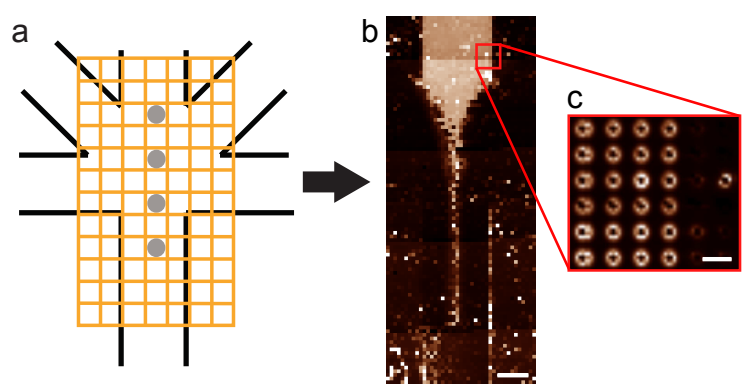

Figure 6.3: Data acquisition and analysis. (a) Scheme of data acquisition in a mesh geometry (not to scale), the gray circles indicate typical analyzed positions. (b) The whole mesh is displayed in a dark-field image with the total scattered intensity in each position plotted on a color scale (typical gold colloid measurement in a COC device at ID13). (c) Beyond the intensity value plotted in b, every position contains a full 2D scattering pattern, which was further analyzed by radial integration. Scale bars are 100 and $10 \mu \mathrm{m}$, respectively.

Measurements were performed on a mesh located at the channel cross section, as schematically shown in Fig. 6.3a. Dark-field images were calculated by integrating the complete scattered intensity in each measurement position and plotting it on a color scale, see Fig. 6.3b, in which the focused flow stream can easily be located. Each 'pixel' in the dark-field image represents a full 2D scattering pattern (Fig. 6.3c), which was azimuthally integrated. After integration, a pointwise background subtraction was performed. The calculated intensity $I(q)$ was then plotted against the magnitude of the scattering vector

$$
q=\frac{4 \pi}{\lambda} \sin (\theta)
$$

where $\theta$ is half the scattering angle and $\lambda$ is the wavelength of the radiation. Guinier analysis of the data was performed using the software package PRIMUS (ATSAS, EMBL, Hamburg, Ger- 
many) [49] to retrieve the overall size of the scatterers by determining the radius of gyration $R_{g}$ for solid spheres:

$$
\ln I(q)=\ln I(0)-\frac{R_{g}^{2}}{3} q^{2}
$$

Furthermore, the pair distance distribution function $p(r)$ (pddf) was used to validate the results:

$$
R_{g}^{2}=\frac{\int_{0}^{D_{\max }} r^{2} p(r) d r}{2 \int_{0}^{D_{\max }} p(r) d r} .
$$

The pddf is the distribution of distances between scatterers in a sample. An advantage of the pddf is that it takes the whole scattering curve into account and not only the first few data points as in Guinier's approximation. In the third step, we fitted the form factor $P(q, R)$ using Matlab2017a (The MathWorks, Natick, MA, USA) for solid spheres. The form factor can be written as

$$
P(q, R)=\left[\frac{3 \sin (q R)-q R \cos (q R)}{q R^{3}}\right]^{2} .
$$

This approach also takes the whole curve into account and the fit curve can be compared to the experimental data.

\subsubsection{Flow Simulations}

COMSOL multiphysics 5.2a (COMSOL GmbH, Göttingen, Germany) was used to perform finite element method (FEM) simulations of the flow conditions in the microfluidic devices. The inlet channels were reduced in length in the model to reduce the computational time, but are long enough to ensure equilibrated flow and mixing profiles. Simulations were performed in the laminar flow regime, thus employing the time-independent Navier-Stokes-equation, and no-slip boundary conditions are used at the channel walls. FEM simulations shown here represent the protein experiments using the diffusion coefficients $D_{\text {vim }}=2.2 \times 10^{-11} \mathrm{~m}^{2} \mathrm{~s}^{-1}$ and $D_{\mathrm{KCl}}=1.84 \times 10^{-9} \mathrm{~m}^{2} \mathrm{~s}^{-1}$. The diffusion coefficient for vimentin is an estimate of the upper bound, as it decreases along the channel as the protein assembles. A change in viscosity upon assembly is not included in our simulation, since viscometry measurements show that the viscosity increases only very little during the first seconds of the assembly process [50, 51]. 


\subsection{Results and Discussion}

\subsubsection{Device Characterization}

Kapton was chosen as a "base line" for our comparison, because the material is well established for X-ray experiments [8-10, 13, 22, 26, 29]. This is due to its low and well defined background signal and negligible radiation sensitivity. Thus, Kapton provides a number of advantages when used as window material. However, in a typical microfluidic chamber, Kapton films have to be glued or bonded to other materials, such as stainless steel plates [8-10, 13, 22], PDMS [26], or UV-curable adhesive [29], that are used for defining the channel geometry, because the material is not self-adhesive. Kapton film with an adhesive layer ("Kapton tape") typically leads to a fairly strong background signal itself. An exception are devices produced by laser ablation directly in Kapton and bonded to thermo-sensitive Kapton [52], which require considerable efforts in fabrication. The COC devices we present here, by contrast, are made from one single material such that there are no (glued) interfaces between the layers. We bond the COC layers directly without using solvents or silanes and without an additional plasticization step.

To benchmark the new devices and test if they are suitable for SAXS measurements in general and in particular for SAXS on weakly scattering protein, we here directly compare measurements in microfluidic devices comprising $70 \mu \mathrm{m}$ COC windows or $16 \mu \mathrm{m}$ Kapton windows (total thickness). As a defined test system, gold colloids were used and we performed the experiments at two different synchrotron beamlines to account for differences concerning primary setup geometry, beam intensity, beam size and detectors. Fig. 6.4a shows typical scattering curves of gold colloids in the two device types, measured at ID13, ESRF. An advantage of the beamline is the highly focused beam $\left(2.7 \times 1.7 \mu \mathrm{m}^{2}\right)$, which can be used to exactly map the microfluidic channel. The background subtracted scattering data in the COC device (green) and in the Kapton device (blue) agree very well, even concerning the scattering intensity values. To further benchmark the validity of the data, we performed an analysis of the pddf yielding a radius of $R=11.02 \mathrm{~nm} \pm 0.15 \mathrm{~nm}$. Furthermore, the form factor of solid spheres was fitted to the data (red solid line) and we obtain a radius of $10.55 \mathrm{~nm} \pm 0.06 \mathrm{~nm}$.

The cSAXS beamline at SLS is optimized for SAXS, providing access to very low $q$-values. Again, the data curves for the COC and the Kapton device fall exactly onto each other, as shown in Fig. 6.4b. In this case, we used colloids with a radius of $15 \mathrm{~nm}$. For these data, we were able to analyze the Guinier region at small $q$-values; we find a colloid radius of $17.88 \mathrm{~nm} \pm 0.49 \mathrm{~nm}$. The pddf yields a radius of $16.50 \mathrm{~nm} \pm 0.04 \mathrm{~nm}$ and the direct fit $R=16.13 \mathrm{~nm} \pm 0.03 \mathrm{~nm}$ (red line). From these experiments, using a simple model system as scatterers, we conclude that the COC devices are well suited for performing SAXS experiments in microflow. 

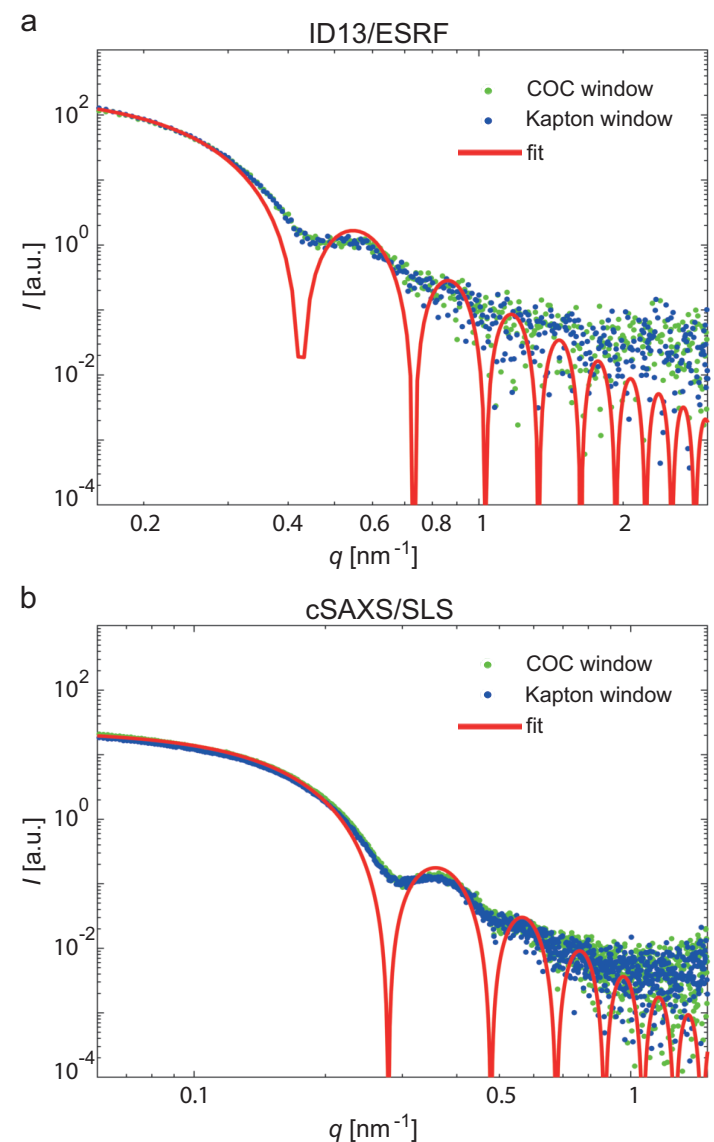

Figure 6.4: Colloid scattering curves with corresponding form factor. (a) Gold colloids measured at the ID13 beamline (ESRF) in a device with a COC window (green) and a Kapton window (blue). The form factor for solid spheres was fitted to the data and we obtain $R=10.55 \mathrm{~nm} \pm 0.06 \mathrm{~nm}$ (red line). (b) Gold colloids measured at the cSAXS beamline (SLS) in a device with a COC window (green) and a Kapton window (blue). The form factor for solid spheres was fitted to the data and we obtain $R=16.13 \mathrm{~nm} \pm 0.03 \mathrm{~nm}$ (red line).

Experiments at synchrotron sources, i.e. with limited available time and highly complex setups, require robust, long-lasting microfluidic devices. In our experience the COC devices are very reliable and outperform the UV-curable adhesive/Kapton devices. It is even possible to flush them by hand, i.e. at comparatively high pressure and little control, without breaking them. In combination with our sample holder (see Fig. $\mathrm{S} 3 \dagger$ ), the success rate for these devices is close to hundred percent. With respect to radiation damage, we observe a strong effect in the Kapton devices, which is, however, not due to the Kapton but to the UV-curable adhesive. In the COC devices the effect is much weaker. We will thus, in the future, investigate radiation resistance of the material in a more systematic way. 


\subsubsection{Protein in Flow}

In a next step, we use our COC devices for measurements on a more challenging system: the weakly scattering protein vimentin. Vimentin belongs to the family of intermediate filament (IF) proteins and is found in cells of mesenchymal origin. Together with actin filaments and microtubules, it forms a composite biopolymer network in the cytoplasm. In vitro, vimentin assembles from tetrameric subunits into extended filaments in a hierarchical manner upon the addition of $\mathrm{KCl}$ [53]. Within the first seconds, lateral assembly of the tetramers leads to so-called unit length filaments (ULFs), followed by an elongation mechanism, which may take minutes to hours and leads to micrometer long filaments [21, 54, 55]. As a critical $\mathrm{KCl}$ concentration for assembly at these high protein concentrations, $10 \mathrm{mM}$ was previously identified [23]. By contrast to previous experiments $[21,23]$, we here realize fast mixing by strong hydrodynamic focusing of the central protein stream (see Fig. 6.3b) $[4,56]$. Thus, the width of the central stream is about $10 \mu \mathrm{m}$ and the diffusion time scale of the $\mathrm{KCl}$ into the central stream is on the order of $10 \mathrm{~ms}$. The rapid increase of the $\mathrm{KCl}$ concentration, as obtained from the FEM simulations, is presented in Fig. 6.5a. The color code of the individual data points corresponds to the positions in Fig. 6.2a and the data curves in Fig. 6.5b. The red curve shows the unassembled tetrameric state, whereas the green curve indicates the start of the assembly.

We observe that the forward scattering $I_{0}$ increases with increasing assembly time, indicating an increase in molecular weight and, thus, assembly. Furthermore, the scattering curves steepen as the assembly proceeds. This change can be interpreted as an increase in thickness of the rod-shaped scatterers, corresponding to the known lateral assembly step of vimentin and in agreement with previously published work $[21,55]$. Notably, we capture very early time points in this particular experiment.

\subsection{Conclusions}

To conclude, we present and characterize a microfluidic device fully made of COC. We demonstrate that the device is well-suited for X-ray measurements in flow. Weak SAXS signals from proteins, even of minute sample volumes, may be detected in a reliable, high-quality way. For benchmarking, we directly compare the performance of the COC devices to devices including Kapton windows. Aside from leading to SAXS data of equal quality, the COC devices provide a number of advantages over the UV-curable adhesive/Kapton devices. Above all, they are fabricated solely from one material, i.e. one type of COC without bonding layers, thus avoiding any gluing or interfaces, which are typically prone to leakage. Instead of plasticizing a thin layer 

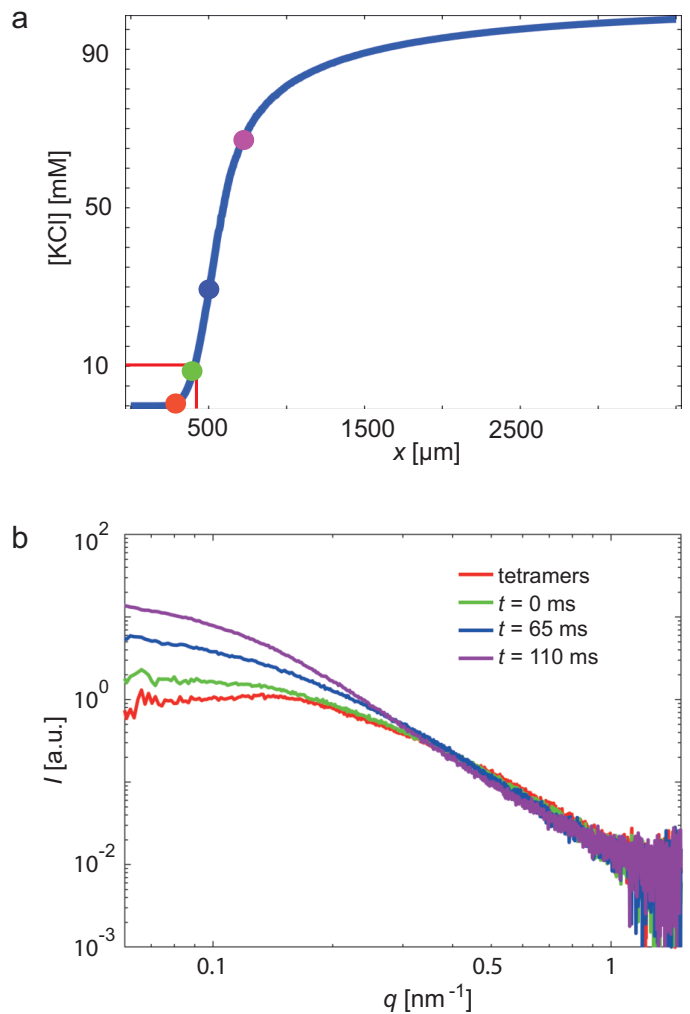

Figure 6.5: Vimentin measurement in a COC device, performed at the cSAXS beamline, SLS. In total, four different positions (see Fig. 6.2a) in the scanned device are shown. (a) The KCl concentration along the central channel is shown. The four analyzed positions are indicated by colored circles. Assembly starts at a KCl concentration of $10 \mathrm{mM}$. (b) The red data curve corresponds to tetrameric vimentin; the green data curve is defined as the starting point of the assembly process $(t=0)$; blue and purple data curves refer to slightly later, yet early, time points.

on top of the COC sheet, we heat up the whole ensemble of two COC layers well above their glass transition temperature. As the unstructured sealing layer is much thinner $(20 \mu \mathrm{m})$ than the structured main layer $(240 \mu \mathrm{m})$ its viscosity decreases more rapidly, allowing for strong bonding. Indeed, the devices are more reliable than other devices we have tested here or in the past. Furthermore, COC materials have a high X-ray transmission and resist radiation. The device fabrication is straightforward and very reproducible at high quality, as it is already established for PDMS-glass devices. By contrast to commercial devices, as used for example in protein crystallography, the channel design is highly adaptable and flexible. Due to the employed lithography methods, the design of the microfluidic device can easily be customized. We believe that this approach will enable the community to perform various X-ray/microfluidics experiments in a very reliable way. Additionally, we are able to show that even weakly scattering protein signals may be investigated in this type of device. We thus expect a large number of different areas of application of these devices in the fields of biophysics, molecular biology and biochemistry. 


\subsection{Acknowledgments}

This work was financially supported by the Deutsche Forschungsgemeinschaft (DFG) in the framework of SFB 755, "Nanoscale Photonic Imaging”, projects B07 and C10, and the Cluster of Excellence and DFG Research Center "Nanoscale Microscopy and Molecular Physiology of the Brain" (CNMPB). The authors thank Susanne Bauch for preparing the protein and TOPAS Advanced Polymers GmbH, Frankfurt, Germany, for providing the COC for the sealing layer. We furthermore thank Thomas Pfohl for helpful discussions. 


\subsection{Supplementary Information}

\section{Device Fabrication (Figure S1)}

In this section, we present more details on our device fabrication method. Fig. S1 (following page) is an extension of Fig. 1 in the main text. All steps, starting from the silicon-wafer with channel structure all the way to the finished device, are shown. 
a
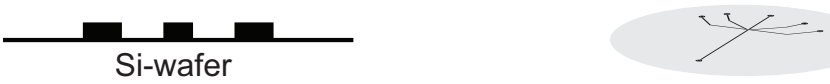

Si-wafer

b
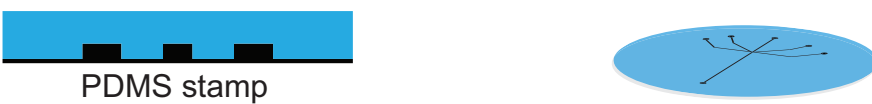

$$
\text { UV-curable adhesive }
$$

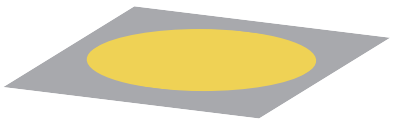

d
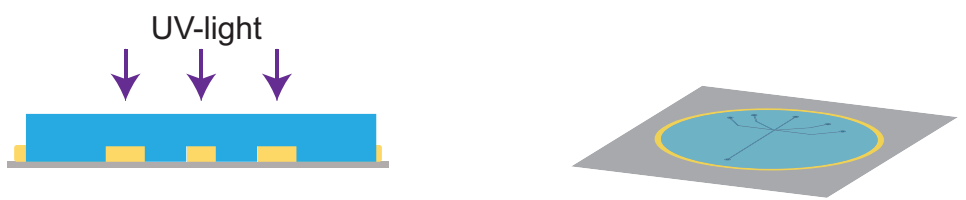

e
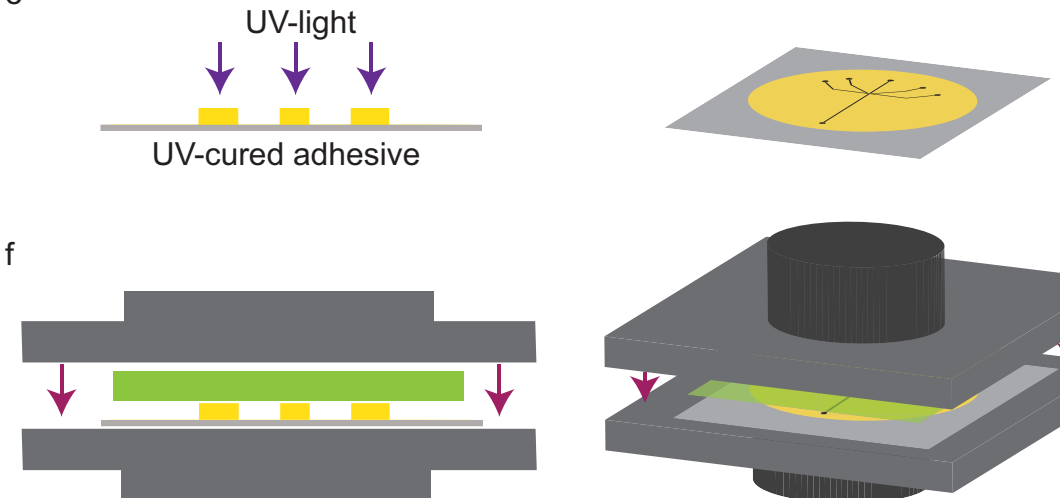

hot press

g
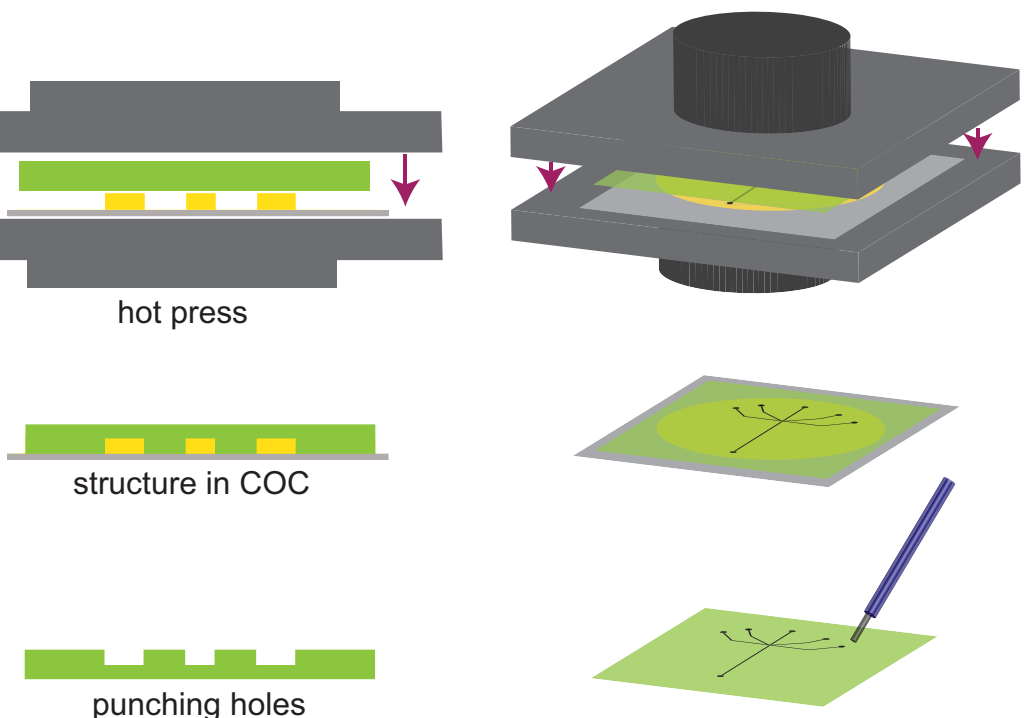

punching holes

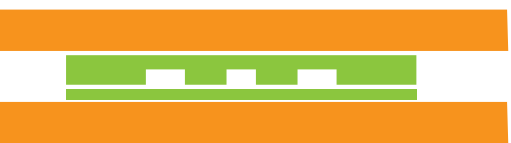

lamination machine

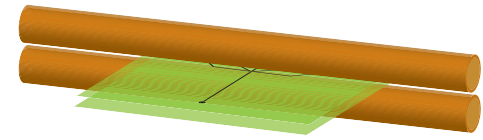

j

finished device

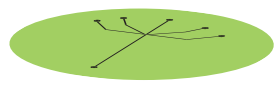


Figure S1 Device Fabrication steps (a) The channel structures (black) are defined by photolithography using SU8-2150 negative photo resist spin coated on Si-wafers to a final height of $160 \mu \mathrm{m}$. (b) PDMS copies (blue) of the channel structures are produced. PDMS and crosslinker are mixed at a ratio of 10:1 and poured onto the structured Si-wafers. After evacuating the PDMS for 20 min to remove all air bubbles, it is cured by backing for $1 \mathrm{~h}$ at $65^{\circ} \mathrm{C}$. (c) A 1 $\mathrm{mm}$ thick glass slide (gray) is prepared with UV-curable adhesive (yellow) and all air bubbles are removed with a needle. (d) The PDMS stamp is pressed into the adhesive and cured with UV-light ( $\lambda=366 \mathrm{~nm}$ ) for three minutes. (e) After detaching the PDMS stamp from the glass slide and removing residual structures with a scalpel, the UV-curable adhesive is cured again for three minutes. (f) The stamp (yellow) and a COC sheet (green) are sandwiched between two sheets of paper, followed by two sheets of aluminum foil. This assembly is heated above the glass transition temperature of the COC $\left(130^{\circ} \mathrm{C}\right)$ for 5 minutes, with the help of a hot press. It is then pressed with initially $2.2 \mathrm{kN}$ for 10 minutes. Thus, the structured UV-cured adhesive is transferred into the COC layer. (g) After cooling down the hot press, the stamp is removed from the COC after wetting with 2-propanol, which flows between the two layers because of capillary forces. The adhesive stamp can be reused. (h) Holes for the inlet and outlet are punched with a biopsy puncher (diameter $0.5 \mathrm{~mm}$ ). (i) A $20 \mu \mathrm{m}$ thick COC sealing layer and the channel structure in COC are sandwiched in paper and placed in a lamination machine with heat and pressure control. The device is sealed at a temperature of $100^{\circ} \mathrm{C}$ and a speed of $7.6 \times 10^{-3} \mathrm{~m} \mathrm{~s}^{-1}$. (j) The finished COC device contains channels with a width of $200 \mu \mathrm{m}$, a height of $160 \mu \mathrm{m}$ and a total thickness of $70 \mu \mathrm{m}$ COC within the beam path. 


\section{Cross Section of the Channel Structure (Figure S2)}

The cross sectional view of a typical device is depicted in Figure S2. Due to the challenge in cutting the device precisely without squeezing the channels the edges appear blurry and the bottom of the device is slightly stretched. However, the well-defined channel structure with straight walls is clearly visible.

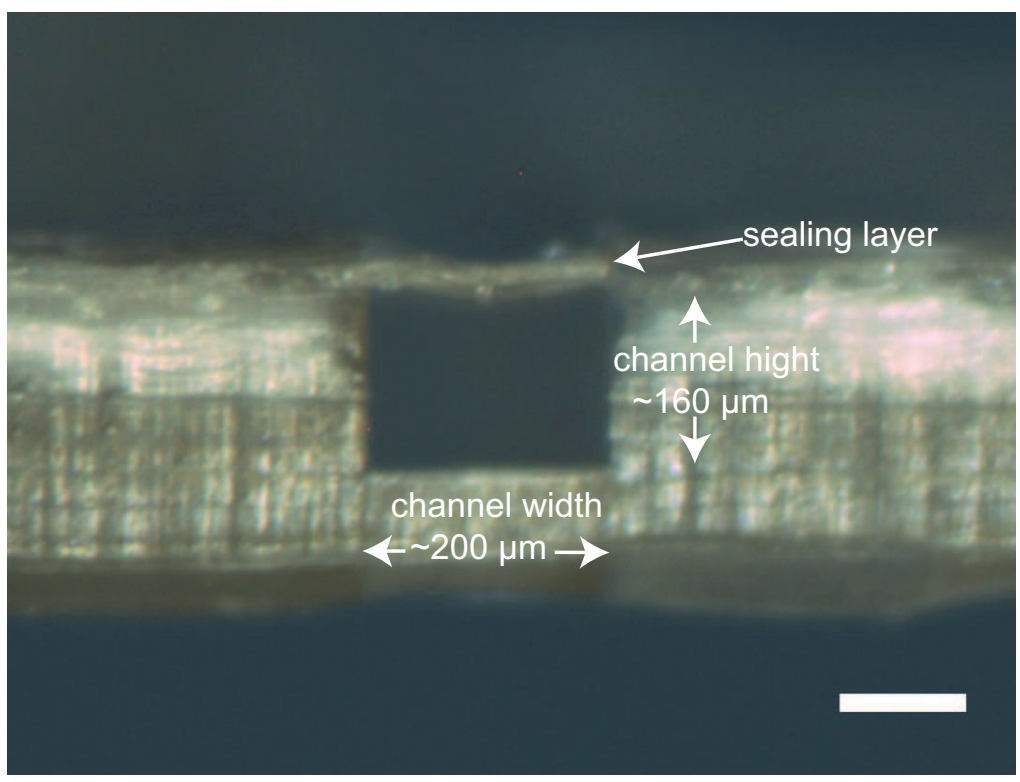

Figure S2 Side view of a typical COC device. The channel height is $\sim 160 \mu \mathrm{m}$ and the channel width $\sim 200 \mu \mathrm{m}$. The scale bar corresponds to $100 \mu \mathrm{m}$. 


\section{Sample Holder (Figure S3)}

In the following the used sample holder is described and shown in detail.

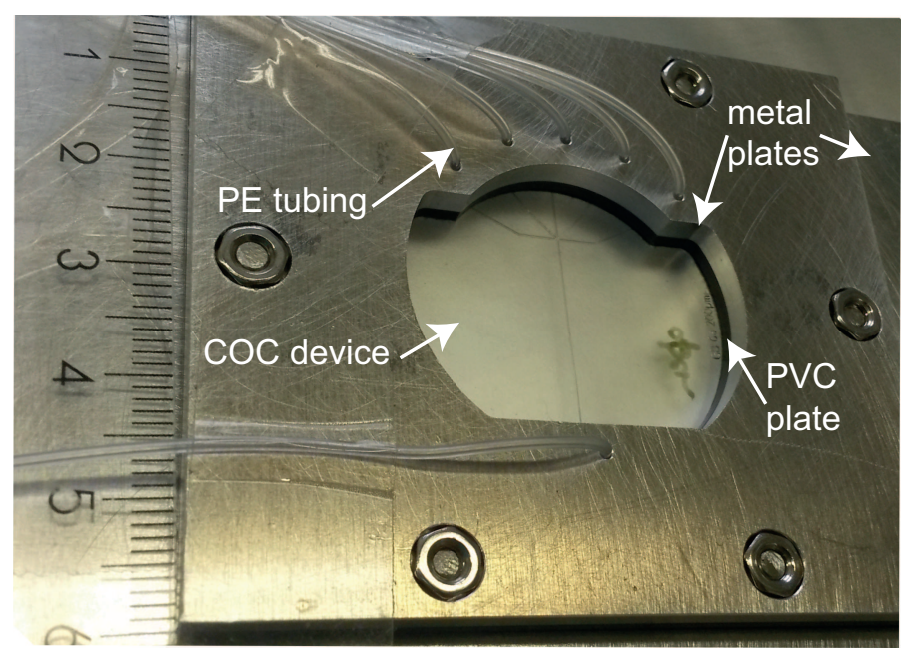

Figure S3 The sample holder consists of two metal plates with different sizes. The metal back plate is larger to allow for mounting to the setup. The upper metal plate contains openings for the tubing. Both plates contain an opening to perform measurements inside the COC device. Sandwiched between the upper metal plate and the COC device is a PVC plate that contains holes for the tubing and indentations for o-rings. The o-rings get compressed by tightening six screws and therefore seal the connection between the device and the tubing. The scale shown is in $\mathrm{cm}$. 


\section{References}

1. Duffy, D. C., McDonald, J. C., Schueller, O. J. A. \& Whitesides, G. M. Rapid Prototyping of Microfluidic Systems in Poly(dimethylsiloxane). Anal. Chem. 70. PMID: 21644679, 49744984 (1998).

2. Ghazal, A. et al. Recent advances in X-ray compatible microfluidics for applications in soft materials and life sciences. Lab Chip 16, 4263-4295 (22 2016).

3. Köster, S. \& Pfohl, T. X-Ray Studies of Biological Matter in Microfluidic Environments. Mod. Phys. Lett. B 26, 1230018 (2012).

4. Pollack, L. et al. Compactness of the denatured state of a fast-folding protein measured by submillisecond small-angle x-ray scattering. P. Natl. Acad. Sci. U.S.A. 96, 10115-10117 (1999).

5. Pollack, L. et al. Time Resolved Collapse of a Folding Protein Observed with Small Angle X-Ray Scattering. Phys. Rev. Lett. 86, 4962-4965 (21 2001).

6. Segel, D. J. et al. Characterization of transient intermediates in lysozyme folding with timeresolved small-angle X-ray scattering 11Edited by A. R. Fersht. J. Mol. Biol. 288, 489-499. ISSN: 0022-2836 (1999).

7. Akiyama, S. et al. Conformational landscape of cytochrome c folding studied by microsecond-resolved small-angle x-ray scattering. P. Natl. Acad. Sci. U.S.A. 99, 1329-1334 (2002).

8. Uzawa, T. et al. Collapse and search dynamics of apomyoglobin folding revealed by submillisecond observations of $\alpha$-helical content and compactness. P. Natl. Acad. Sci. U.S.A. 101, 1171-1176 (2004).

9. Uzawa, T. et al. Time-resolved Small-angle X-ray Scattering Investigation of the Folding Dynamics of Heme Oxygenase: Implication of the Scaling Relationship for the Submillisecond Intermediates of Protein Folding. J. Mol. Biol. 357, 997-1008. ISSN: 0022-2836 (2006).

10. Arai, M. et al. Microsecond Hydrophobic Collapse in the Folding of Escherichia coli Dihydrofolate Reductase, an $\alpha / \beta$-Type Protein. J. Mol. Biol. 368, 219-229. ISSN: 0022-2836 (2007).

11. Wu, Y., Kondrashkina, E., Kayatekin, C., Matthews, C. R. \& Bilsel, O. Microsecond acquisition of heterogeneous structure in the folding of a TIM barrel protein. P. Natl. Acad. Sci. U.S.A. 105, 13367-13372 (2008).

12. Arai, M., Iwakura, M., Matthews, C. R. \& Bilsel, O. Microsecond Subdomain Folding in Dihydrofolate Reductase. J. Mol. Biol. 410, 329-342. ISSN: 0022-2836 (2011). 
13. Graceffa, R. et al. Sub-millisecond time-resolved SAXS using a continuous-flow mixer and X-ray microbeam. J. Synchrotron Radiat. 20, 820-825 (2013).

14. Russell, R. et al. Rapid compaction during RNA folding. P. Natl. Acad. Sci. U.S.A. 99, 42664271 (2002).

15. Das, R. et al. The Fastest Global Events in RNA Folding: Electrostatic Relaxation and Tertiary Collapse of the Tetrahymena Ribozyme. J. Mol. Biol. 332, 311-319. ISSN: 0022-2836 (2003).

16. Kwok, L. W. et al. Concordant Exploration of the Kinetics of RNA Folding from Global and Local Perspectives. J. Mol. Biol. 355, 282-293. ISSN: 0022-2836 (2006).

17. Lamb, J. et al. Reconstructing three-dimensional shape envelopes from time-resolved small-angle X-ray scattering data. J. Appl. Crystallogr. 41, 1046-1052 (2008).

18. Schlatterer, J. C. et al. Hinge Stiffness Is a Barrier to RNA Folding. J. Mol. Biol. 379, 859-870. ISSN: 0022-2836 (2008).

19. Dootz, R., Otten, A., Köster, S., Struth, B. \& Pfohl, T. Evolution of DNA compaction in microchannels. J. Phys.: Condens. Matter 18, 639-652 (2006).

20. Pfohl, T. et al. Highly Packed and Oriented DNA Mesophases Identified Using in Situ Microfluidic X-ray Microdiffraction. Biomacromolecules 8. PMID: 17579478, 2167-2172 (2007).

21. Brennich, M. E. et al. Dynamics of intermediate filament assembly followed in micro-flow by small angle X-ray scattering. Lab Chip 11, 708-716 (2011).

22. Köster, S., Evans, H. M., Wong, J. Y. \& Pfohl, T. An in-situ Study of Collagen Self-Assembly Processes. Biomacromolecules 9, 199-207 (2008).

23. Saldanha, O., Brennich, M. E., Burghammer, M., Herrmann, H. \& Köster, S. The filament forming reaction of vimentin tetramers studied in a serial-inlet microflow device vy small angle x-ray scattering. Biomicrofluidics 10, 24108 (2016).

24. Saldanha, O. et al. Rapid Acquisition of X-Ray Scattering Data from Droplet-Encapsulated Protein Systems. ChemPhysChem 18, 1220-1223. ISSN: 1439-7641 (2017).

25. Stehle, R., Goerigk, G., Wallacher, D., Ballauff, M. \& Seiffert, S. Small-angle X-ray scattering in droplet-based microfluidics. Lab Chip 13, 1529-1537 (2013).

26. Dootz, R., Evans, H., Köster, S. \& Pfohl, T. Rapid Prototyping of X-Ray Microdiffraction Compatible Continuous Microflow Foils. Small 3, 96-100 (2007).

27. Trebbin, M. et al. Anisotropic particles align perpendicular to the flow direction in narrow microchannels. P. Natl. Acad. Sci. U.S.A. 110, 6706-6711 (2013). 
28. Evans, H., Dootz, R., Köster, S. \& Pfohl, T. X-ray microdiffraction on flow-controlled biomolecular assemblies. B. Pol. Acad. Sci.-Tech. 55, 217-227 (2007).

29. Weinhausen, B. \& Köster, S. Microfluidic devices for X-ray studies on hydrated cells. Lab Chip 13, 212-215 (2 2013).

30. Steigert, J. et al. Rapid prototyping of microfluidic chips in COC. J. Micromech. Microeng. 17, 333-341 (2007).

31. Steinert, C. P. et al. Miniaturized and highly parallel protein crystallization on a microfluidic disc in 2007 IEEE 20th International Conference on Micro Electro Mechanical Systems (MEMS) (2007), 561-564.

32. Ng, J. D., Clark, P. J., Stevens, R. C. \& Kuhn, P. In situ X-ray analysis of protein crystals in lowbirefringent and X-ray transmissive plastic microchannels. Acta Crystallogr. D64, 189-197 (2008).

33. Guha, S., Perry, S. L., Pawate, A. S. \& Kenis, P. J. Fabrication of X-ray compatible microfluidic platforms for protein crystallization. Sens Actuators B Chem 174. 23105172[pmid], 1-9. ISSN: 0925-4005 (2012).

34. Maeki, M. et al. A Method of Cryoprotection for Protein Crystallography by Using a Microfluidic Chip and Its Application for in Situ X-ray Diffraction Measurements. Anal. Chem. 87. PMID: 25834918, 4194-4200. eprint: http://dx . doi .org/10.1021/acs . analchem . $5 \mathrm{~b} 00151$ (2015).

35. Pawate, A. S. et al. Towards time-resolved serial crystallography in a microfluidic device. Acta Crystallogr. F 71, 823-830 (2015).

36. Silva, B. F. B. et al. Nematic Director Reorientation at Solid and Liquid Interfaces under Flow: SAXS Studies in a Microfluidic Device. Langmuir 31. PMID: 25396748, 4361-4371. eprint: http://dx.doi.org/10.1021/la5034614 (2015).

37. Aghvami, S. A. et al. Rapid prototyping of cyclic olefin copolymer (COC) microfluidic devices. Sensors and Actuators B: Chemical 247, 940-949. ISSN: 0925-4005 (2017).

38. I Wallow, T. et al. Low-distortion, high-strength bonding of thermoplastic microfluidic devices employing case-II diffusion-mediated permeant activation. Lab Chip 7, 1825-31 (2008).

39. Uba, F. I., Hu, B., Weerakoon-Ratnayake, K., Oliver-Calixte, N. \& Soper, S. A. High process yield rates of thermoplastic nanofluidic devices using a hybrid thermal assembly technique. Lab Chip 15, 1038-1049 (4 2015). 
40. Cherdron, H., Brekner, M.-J. \& Osan, F. Cycloolefin-copolymere: Eine neue klasse transparenter thermoplaste. Angew. Makromol. Chem. 223, 121-133. Issn: 1522-9505 (1994).

41. Quake, S. R. \& Scherer, A. From Micro- to Nanofabrication with Soft Materials. Science 290, 1536-1540. ISSN: 0036-8075 (2000).

42. Park, H. Y. et al. Achieving Uniform Mixing in a Microfluidic Device: Hydrodynamic Focusing Prior to Mixing. Anal. Chem. 78. PMID: 16808455, 4465-4473. eprint: http: //dx . doi . org/10.1021/ac060572n (2006).

43. Urbani, R. B. Dynamics in Microfluidics Measured by X-Ray Scattering Techniques PhD thesis (Philosophisch-Naturwissenschaftliche Fakultät, Universität Basel, 2015).

44. Herrmann, H., Kreplak, L. \& Aebi, U. in Intermediate Filament Cytoskeleton (eds Omary, M. B. \& Coulombe, P. A.) 3-24 (Academic Press, 2004).

45. Nöding, B. \& Köster, S. Intermediate Filaments in Small Configuration Spaces. Phys. Rev. Lett. 108 (2012).

46. Winheim, S. et al. Deconstructing the Late Phase of Vimentin Assembly by Total Internal Reflection Fluorescence Microscopy (TIRFM). PLOS ONE 6 (ed Kreplak, L.) e19202 (2011).

47. Dinapoli, R. et al. EIGER: Next generation single photon counting detector for X-ray applications. Nucl. Instrum. Meth. A 650. International Workshop on Semiconductor Pixel Detectors for Particles and Imaging 2010, 79-83. ISSN: 0168-9002 (2011).

48. Henrich, B. et al. PILATUS: A single photon counting pixel detector for X-ray applications. Nucl. Instrum. Meth. A 607, 247-249. ISSN: 0168-9002 (2009).

49. Konarev, P. V., Volkov, V. V., Sokolova, A. V., Koch, M. H. J. \& Svergun, D. I. PRIMUS: a Windows PC-based system for small-angle scattering data analysis. J. Appl. Crystallogr. 36, 1277-1282 (2003).

50. Herrmann, H. et al. Structure and Assembly Properties of the Intermediate Filament Protein Vimentin: The Role of its Head, Rod and Tail Domains. J. Mol. Biol. 264, 933-953 (1996).

51. Hofmann, I., Herrmann, H. \& Franke, W. W. Assembly and structure of calcium-induced thick vimentin filaments. Eur. J. Cell Biol. 56, 328-341 (1991).

52. Barrett, R. et al. X-ray microfocussing combined with microfluidics for on-chip X-ray scattering measurements. Lab Chip 6, 494-499 (4 2006).

53. Mücke, N. et al. Molecular and Biophysical Characterization of Assembly-Starter Units of Human Vimentin. J. Mol. Biol. 340, 97-114 (2004). 
54. Lopez, C. G., Saldanha, O., Huber, K. \& Köster, S. Lateral association and elongation of vimentin intermediate filament proteins: A time-resolved light-scattering study. P. Natl. Acad. Sci. U.S.A. 113, 11152-11157 (2016).

55. Herrmann, H., Bär, H., Kreplak, L., Strelkov, S. V. \& Aebi, U. Intermediate filaments: from cell architecture to nanomechanics. Nat. Rev. Mol. Cell Bio. 8, 562-573 (2007).

56. Knight, J. B., Vishwanath, A., Brody, J. P. \& Austin, R. H. Hydrodynamic Focusing on a Silicon Chip: Mixing Nanoliters in Microseconds. Phys. Rev. Lett. 80, 3863-3866 (1998). 


\section{Chapter 7}

\section{Summary, Discussion and Outlook}

The cell contains a plethora of different ions which are necessary to maintain certain functions in the cell. The presence of ions does not only affect the function of proteins but can also, for example trigger their assembly. It can, however, occur that instead of the fully functional protein, aggregates are formed. Thus, it is important to investigate the influence of different ions on proteins to understand their fundamental effects on the assembly. As the cell itself contains many different proteins, which commonly interact with each other, in vitro studies can help to understand the physical and chemical properties of each protein type on its own.

In previous in vitro studies it has been shown that ions have an influence on IFs. Whereas the presence of, e.g. monovalent ions, can initiate the assembly of intermediate filaments [1-3], the addition of multivalent ions to already formed filaments leads to network formation, bundling or gelation [4-6]. It has furthermore been shown that divalent ions can induce the assembly of vimentin protein. Differences in the filament radius, the filament homogenities, as well as the threshold for precipitation were found [7-9]. In the mentioned studies, only one or two ion types are investigated. In this thesis, the assembly of vimentin protein in the presence of six different ions, with varying valencies, are measured and compared in a systematic way. Using small angle $\mathrm{X}$-ray scattering the lateral assembly of the protein is investigated. The radius of the filament as well as the molecular weight can be retrieved. Using microscopy techniques (AFM and fluorescence microscopy) it is observed if single filaments or networks are formed in the presence of the ions.

In vitro, vimentin protein studies are usually carried out in the $\mathrm{pH}$-buffers TRIS and $\mathrm{PB}[3,10]$. However, both buffers can complex with certain ions and therefore are not usable in studies where the effects of different ions on the assembly of vimentin are investigated. Thus, a suitable buffer system needs to be found. Good et al.proposed different criteria for buffers and introduced several so called Good buffers [11, 12]. According to the criteria for a Good buffer, both 
TRIS and PB do not fulfill the criteria, and therefore, the Good buffer MOPS is chosen to perform the assembly experiments [12]. Prior to the experiments to study the influence of ions on the assembly of vimentin protein, the buffer needs to be tested to determine its suitability for vimentin assembly studies. Therefore, vimentin tetramers and filaments are investigated in MOPS buffer and compared to measurements performed in TRIS and PB. As both TRIS and PB should have no influence on vimentin protein, this is a good way to see if MOPS interacts with vimentin.

Each buffer is tested at low $(2 \mathrm{mM})$ and high $(20 \mathrm{mM})$ concentration. The higher the buffer concentration the better the buffering capacity, however the more likely the buffer interacts with the protein. At low buffer concentration no difference regarding the radius and the molecular weight between the tetrameric state as well as the assembled filaments can be observed. The found values for the radius of the filaments are in agreement with the literature [7]. In the AFM images, vimentin protein forms single filaments upon addition of $100 \mathrm{mM} \mathrm{KCl}$ in the three different buffers at low buffer concentration as described in literature [3]. When vimentin protein is investigated at the high buffer concentration it can be observed that already the tetrameric state of vimentin protein shows pre-assembled or aggregated protein. Upon addition of $100 \mathrm{mM}$ $\mathrm{KCl}$ filaments with a slightly larger radius compared to the filaments in the low buffer concentrations are formed in all three buffers.

By investigating the tetrameric as well as the filamental state of vimentin protein in the three different buffers at a buffer concentration of $2 \mathrm{mM}$ no differences can be observed. Thus, $2 \mathrm{mM}$ MOPS could be established as a new buffer for vimentin studies.

As no significant difference between vimentin in the tetrameric and filamental state in the three buffers at low buffer concentration can be detected, the influence of ions on the assembly of vimentin filaments is investigated in MOPS buffer. In total the influence of six different ions $\left(\mathrm{Na}^{+}\right.$, $\mathrm{K}^{+}, \mathrm{Mg}^{2+}, \mathrm{Ca}^{2+}, \mathrm{Co}^{3+}$ and $\mathrm{Sp}^{4+}$ ) on the assembly of vimentin protein is investigated with SAXS at eight different concentrations. Fluorescence microscopy of vimentin filaments at one ion concentration is conducted to detect if single filaments or networks are formed. The ions are chosen to have two monovalent and two divalent ions to investigate if there are differences in the assembly of vimentin filaments using different ions with the same valency. Furthermore, vimentin filaments are assembled with a trivalent ion and a tetravalent molecule to investigate the effects of different ion valencies on the assembly of vimentin filaments. For analyzing the SAXS data, different analyzing methods (model-free and model-based) are applied to retrieve the radius of the filament, the radius of gyration of the tails from the filaments, information about the subunits in the filament, as well as the amount of tetramers in the system.

In general it can be observed that with increasing ion concentration the radius of the filament and the number of subunits per cross-section increase. Furthermore, the amount of tetramers 
in the solution decreases with increasing ion concentration, as expected, as at higher concentration more protein should assemble into filaments. In the case of vimentin assembled in the presence of the monovalent ions $\mathrm{KCl}$ and $\mathrm{NaCl}$, no differences could be observed. In both cases the radii retrieved of the filament correspond to values found in the literature $[7,13]$. Furthermore, single filaments are formed in the presence of $100 \mathrm{mM}$ salt as described in the literature [3]. Comparing the results found for vimentin protein assembled in the presence of $\mathrm{Mg}^{2+}$, similar values for the radius are found when compared to the literature [7]. When vimentin is assembled in the presence of the divalent ions $\mathrm{Ca}^{2+}$ and $\mathrm{Mg}^{2+}$ it can be observed that in both cases vimentin precipitates after exceeding a certain ion concentration. The threshold for the precipitation of vimentin assembled in the presence of $\mathrm{Ca}^{2+}$ is lower $(2.5 \mathrm{mM}-4 \mathrm{mM})$ than the threshold found for vimentin in the presence of $\mathrm{Mg}^{2+}(5 \mathrm{mM}-10 \mathrm{mM})$. Assembling vimentin filaments with divalent ions ( $4 \mathrm{mM} \mathrm{Mg}^{2+}$ and $2 \mathrm{mM} \mathrm{Ca}^{2+}$ ), it can be observed that the ions have the ability to cross-link filaments and form networks, as described in literature [8]. Comparing the results from the experiments with the divalent ions, it can be concluded that less $\mathrm{Ca}^{2+}$ is needed for the same effect on vimentin protein as $\mathrm{Mg}^{2+}$. Vimentin filaments assembled with the trivalent ion $\mathrm{Co}^{3+}$ show again precipitation of the sample at high ion concentrations (threshold for precipitation: $0.1 \mathrm{mM}-0.2 \mathrm{mM}$ ), which is lower than the concentration found for divalent ions. In the microscopy experiments vimentin filaments again form networks, showing that also $\mathrm{Co}^{3+}$ has the ability to cross-link filaments. Compared to divalent ions, less $\mathrm{Co}^{3+}$ is needed to initiate assembly and precipitation. Finally, the influence of the tetravalent molecule spermine on the assembly of vimentin filaments is investigated. Again, vimentin protein starts to precipitate after a certain $\mathrm{Sp}^{4+}$ concentration is exceeded $(0.05 \mathrm{mM}-0.08 \mathrm{mM})$. The threshold for aggregation is lower than for all the other ions investigated. Networks are observed in microscopy experiments when vimentin protein is assembled with $\mathrm{Sp}^{4+}$, indicating that also $\mathrm{Sp}^{4+}$ has the ability to cross-link vimentin filaments.

Combining all results, it can be observed that vimentin filaments assembled with monovalent ions form single filaments. On the contrary, filaments assembled with all multivalent ions investigated here show the ability to cross-link and, furthermore, induce precipitation of the protein. Normally, two filaments with the same net negative charge would repel each other. However, due to the effect of counterion condensation on the filaments, an arrangement of screening ions surrounding the filament is induced, resulting in the attraction between the filaments which leads to network formation. The higher the valency of the ion, the lower the amount of ions on the surface of the filament is needed for network formation. The networks formed with $\mathrm{Mg}^{2+}$ are large compared to vimentin networks assembled with $\mathrm{Ca}^{2+}$, and even smaller and fewer networks are found assembling vimentin with $\mathrm{Co}^{3+}$ or $\mathrm{Sp}^{4+}$. One reason could be that trivalent and tetravalent ions can mediate more interactions between filaments due to the higher number of 
charges. This could lead to smaller but more densely packed networks. A different reason for the smaller networks could be that there is a larger network which was not detected during the experiments. Besides the ability to cross-link filaments, vimentin precipitated with multivalent ions at higher ion concentrations. The higher the valency of the ion, the lower the ion concentration needed to initiate assembly of the protein and the lower the threshold for precipitation of vimentin protein. A radius of up to $10 \mathrm{~nm}$ is found for vimentin filaments assembled with multivalent ions. Filaments exceeding a filament radius of roughly $10 \mathrm{~nm}$ might start to form very dense networks (precipitation), which are visible by eye.

In addition to the findings for the differences found for the ions of different valencies, a much stronger effect of $\mathrm{Ca}^{2+}$ on vimentin filaments is observed than with $\mathrm{Mg}^{2+}$. Those differences can be explained by the Hofmeister effect, which is often found to describe the interaction of ions with a protein $[8,14]$. The Hofmeister series lists a sequence of ions according to their ability to affect the solubility of proteins. Both $\mathrm{Mg}^{2+}$ and $\mathrm{Ca}^{2+}$ are listed in the series, showing that less $\mathrm{Ca}^{2+}$ is needed to induce the same effect as $\mathrm{Mg}^{2+}$.

Concluding, it could be shown that even upon the addition of different ions, similar observation could be made. In contrast to monovalent ions, all multivalent ions investigated are able to induce cross-linking of the vimentin filaments as well as precipitation (collapse of the filaments to very densly packed networks) of the filaments is observed.

So far in this thesis, only static experiments of the ion influence on the assembly of vimentin filaments are performed. However, it would be interesting to investigate the temporal evolution of vimentin filament assembly as well. By combining SAXS measurements with microfluidics, the assembly process can be followed over time. When combining microfluidics with SAXS, it is necessary to have an appropriate microfluidic device which is resistant to X-ray illumination, it should be X-ray transparent and have a low scattering signal. Many materials can be used to build the microfluidic devices, mostly involving several different materials. Here, microfluidic devices made solely out of the material cyclic olefin copolymer (COC) are used [15] and compared to Kapton/UV-curable adhesive devices [16]. In the Kapton/UV-curable adhesive devices the window material where the X-rays pass is Kapton; a material often used in X-ray studies. However, the Kapton foils are glued together with a UV-curable adhesive, which shows radiation damage. Additionally, the interfaces between Kapton and the adhesive are prone to leakage. The devices are tested by flushing in gold colloids and comparing the signal and data quality between COC devices and Kapton/UV-curable adhesive devices. No difference between the data quality of the recorded signals in both device types could be detected. Even if the signals recorded in both device types are similar, the easier and more straightforward production, as well as the higher stability (less leaking) of the devices, make COC devices better suited for X-ray experiments. In addition to the device testing, vimentin protein is assembled in the microfluidic 
devices and investigated with SAXS. The resulting data of the weakly scattering protein in COC devices are of high quality. By initiating the assembly process of vimentin protein in the device, the early time points (ms range) of the vimentin assembly were investigated. To conclude this part of the thesis, an X-ray compatible microfluidic device solely made out of COC could be introduced. We could show that COC is promising device material for low-scattering samples like vimentin. Furthermore we showed, that the very early time points of vimentin assembly could be investigated.

In the future, the influence of different ions not only on vimentin filaments but also on other IFs, e.g. keratin or desmin, could be investigated. As IFs are expressed in a cell specific manner, different IFs can interact differently with ions. Indeed, it has already been shown that keratin filaments interact differently with $\mathrm{K}^{+}$and $\mathrm{Mg}^{2+}$ compared to vimentin filaments [17]. By comparing the results from different IFs, a general model on the behaviour of IFs with ions could eventually be retrieved. To further invest the temporal evolution of vimentin filaments, a step should be included in the device to avoid the protein sticking to the channels walls. When the assembly starts, the protein is enclosed by buffer and cannot stick to the channel walls. The step in the device has already been used in different vimentin studies and proofed to reliably keep the protein from the walls and with that, the assembly can be investigated [18]. With this device geometry, the temporal evolution of the protein with different ions can be studied and the assembly times of the protein could be retrieved. 


\section{References}

1. Mücke, N., Kirmse, R., Wedig, T., Leterrier, J. \& Kreplak, L. Investigation of the morphology of intermediate filaments adsorbed to different solid supports. J. Struct. Biol. 150, 268-276 (2005).

2. Lopez, C. G., Saldanha, O., Huber, K. \& Köster, S. Lateral association and elongation of vimentin intermediate filament proteins: A time-resolved light-scattering study. P. Natl. Acad. Sci. U.S.A. 113, 11152-11157 (2016).

3. Block, J. et al. Viscoelastic properties of vimentin originate from nonequilibrium conformational changes. Sci. Adv. 4, eaat1161 (2018).

4. Dammann, C., Herrmann, H. \& Köster, S. Competitive Counterion Binding Regulates the Aggregation Onset of Vimentin Intermediate Filaments. Isr. J. Chem. 56, 614-621 (2015).

5. Janmey, P. A., Slochower, D. R., Wang, Y.-H., Wen, Q. \& Cēbers, A. Polyelectrolyte properties of filamentous biopolymers and their consequences in biological fluids. Soft Matter 10, 1439 (2014).

6. Charrier, E. E. \& Janmey, P. A. in Methods in Enzymology 35-57 (Elsevier, 2016).

7. Brennich, M. E. et al. Impact of ion valency on the assembly of vimentin studied by quantitative small angle X-ray scattering. Soft Matter 10, 2059-2068 (2014).

8. Wu, H. et al. Effect of the divalent cations zinc and calcium on the structure and mechanics of reconstituted vimentin intermediate filaments (2019).

9. Hofmann, I., Herrmann, H. \& Franke, W. W. Assembly and structure of calcium-induced thick vimentin filaments. Eur. J. Cell Biol. 56, 328-341 (1991).

10. Mücke, N. et al. Molecular and Biophysical Characterization of Assembly-Starter Units of Human Vimentin. J. Mol. Biol. 340, 97-114 (2004).

11. Good, N. E. et al. Hydrogen Ion Buffers for Biological Research. Biochemistry 5, 467-477 (1966).

12. Ferreira, C. M. H., Pinto, I. S. S., Soares, E. V. \& Soares, H. M. V. M. (Un)suitability of the use of pH buffers in biological, biochemical and environmental studies and their interaction with metal ions - a review. RSC Advances 5, 30989-31003 (2015).

13. Kreplak, L. \& Fudge, D. Biomechanical properties of intermediate filaments: from tissues to single filaments and back. Bioessays 29, 26-35 (2006).

14. Hofmeister, F. Zur Lehre von der Wirkung der Salze. Arch. Exp. Pathol. Pharmakol. 24, 247260 (1888). 
15. Denz, M. et al. Cyclic olefin copolymer as an X-ray compatible material for microfluidic devices. Lab Chip 18, 171-178 (2018).

16. Weinhausen, B. \& Köster, S. Microfluidic devices for X-ray studies on hydrated cells. Lab Chip 13, 212-215 (2 2013).

17. Hémonnot, C. Y. J., Mauermann, M., Herrmann, H. \& Köster, S. Assembly of Simple Epithelial Keratin Filaments: Deciphering the Ion Dependence in Filament Organization. Biomacromolecules 16, 3313-3321 (2015).

18. Brennich, M. E. et al. Dynamics of intermediate filament assembly followed in micro-flow by small angle X-ray scattering. Lab Chip 11, 708-716 (2011). 


\section{Appendix A}

\section{Calculation of the Correction Factor}

\section{A.1 Calculating the Correction Factor}

To get the scattering data on absolute scale $\left(\mathrm{cm}^{-1}\right)$ the Correction Factor (CF) has to be calculated. This needs to be done if data which was taken at different setups wants to be compared. Or if e.g. the molecular weight, volume fraction or the specific surface area of the scatterer wants to be determined [1]. There are many ways to calculate the CF including primary standards (e.g. water), secondary standards (e.g. glassy carbon) or biopolymers with a known scattering signal (e.g. lysozyme) [1-3]. Here, water is used to calculate the CF, even thought it has a low scattering cross-section. Water is widely used and with a sufficient exposure time, the signal is of good quality and it can be used to calculate the CF using the formula:

$$
\mathrm{CF}=\left(\frac{\partial \Sigma}{\partial \Omega}\right)_{s t}(q) \frac{d_{s t} T_{s t+c a p}}{\left[I_{s t}(q)-\mathrm{BG}_{s t}\right]}
$$

where $\frac{\partial \Sigma)}{(\partial \Omega)_{s t}(q)}$ is the differential scattering cross-section, $d_{s t}$ the thickness of the standard, $T_{s t+c a p}$ the transmission of the standard in the capillary, $I_{s t}$ the measured intensity of the standard and $\mathrm{BG}_{s t}$ the background $[1,4,5]$.

Regarding classical fluctuation theory for pure liquids [6], the differential scattering crosssection (in $\mathrm{cm}^{-1}$ ) can be extrapolated to $q=0$ and written as [5]:

$$
\left(\frac{\partial \Sigma}{\partial \Omega}\right)_{s t}(0)=c n_{e}^{2} b_{e}^{2}\left(\rho k_{B} T\right) \chi_{T} .
$$

Were $c$ is the concentration of the scattering objects, $n_{e}$ the number of electrons within the object, $b_{e}$ is the Thomson factor $\left(b_{e}=0.2818 \cdot 10^{-12} \mathrm{~cm}\right), k_{B}$ the Boltzmann constant, $T$ the temperature and $\chi_{T}$ the isothermal compressibility. In literature the differential scattering crosssection for water is given by: 


$$
\left(\frac{\partial \Sigma}{\partial \Omega}\right)_{\mathrm{H}_{2} \mathrm{O}}(0)=1.65 \cdot 10^{-2} \mathrm{~cm}^{-1},
$$

at $T=293 \mathrm{~K}$, a pressure of $10^{-5} \mathrm{~Pa}$ and $\chi_{T}=4.591 \cdot 10^{-10} \mathrm{~Pa}^{-1}[1,5]$. To calculate the CF with water, an empty capillary as well at a water-filled capillary are measured for $24 \mathrm{~h}$. A long exposure is chosen to have a good signal to noise ratio despite the low scattering of both the empty capillary and the water. In literature it is mentioned, that the dark current of the detector needs to be subtracted as well $[1,5]$. However according to the manufacturer of the detector (Dectris Ltd., Baden, Switzerland), there is no dark current due to the single photon counting technology. In Fig. A.1 the scattering curve after background subtraction and correction with the transmission values is shown (red dots).

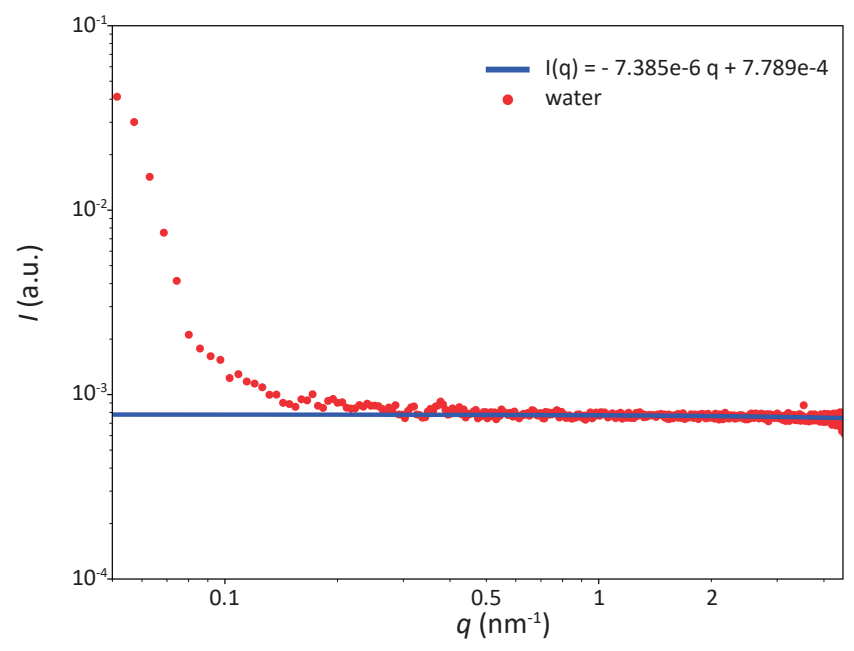

Figure A.1: Scattering profile of water after background subtraction and correction with the transmission values. The water signal is shown in red. The signal shows the for water typical flat part at large q values. The linear extrapolation of the signal at large q values to $I=0$ is shown in blue.

The intensity profile of water is linear at large $q$ values. Extrapolating the large $q$ values to $q=0$ a value of $7.789 \cdot 10^{-4}$ is retrieved for the used setup. After calculating the thickness of the water in the capillary using the transmission values, the CF can be calculated:

$$
C F=\frac{1.65 \cdot 10^{-2}}{7.789 \cdot 10^{-4}} \cdot 0.124=2.62 \mathrm{~cm}^{-1} .
$$

The calculated value of $2.62 \mathrm{~cm}^{-1}$ can be multiplied with each scattering curve taken on that setup to normalize data on absolute scale. The water measurement for CF calculation is performed two times in two different capillaries. The second measurement yields a value of $2.67 \mathrm{~cm}^{-1}$. From both values an arithmetic mean is calculated which is used to normalize the data. 
A CF for the data with the sample chamber in air could not be calculated due to a strong scattering in the flattened part of the curve (Fig A.2a).

(a)

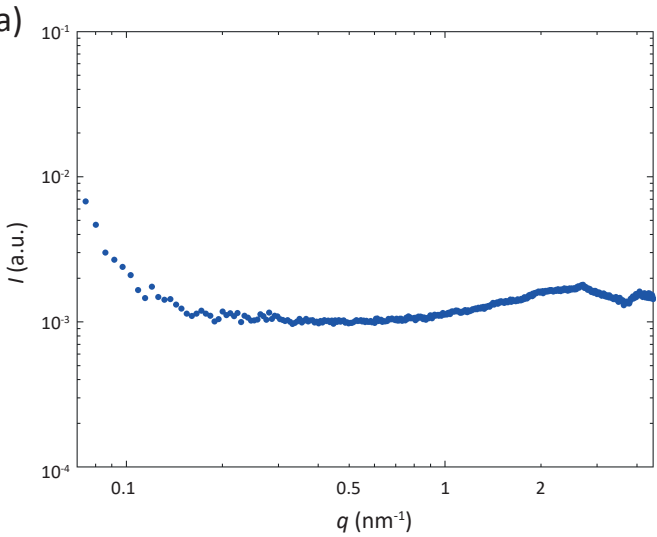

(b)

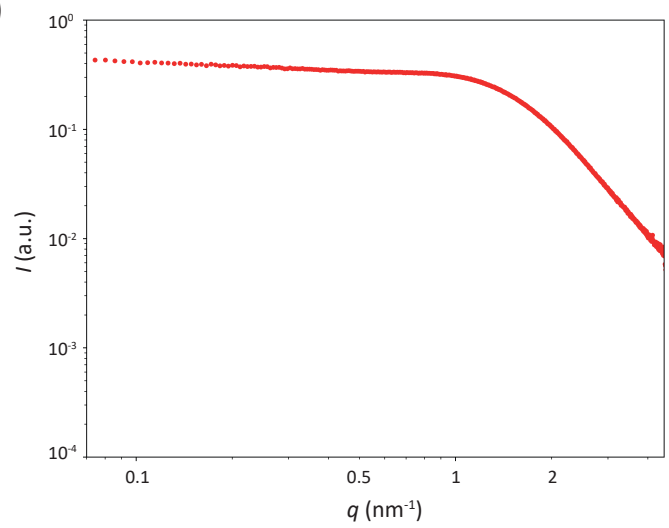

Figure A.2: Scattering profiles of water (without the sample chamber in vacuum) and glassy carbon. (a) Scattering curve of water after background subtraction and correction of the transmission values, when the sample chamber of the setup is in air. (b) Scattering profile of glassy carbon.

It is not clear what causes this scattering at large $q$ values, however, a reason could be the introduction of the two windows, to exclude the sample chamber from vacuum. The protein profiles measured when the whole system is under vacuum or when the sample chamber is in air are very similar and thus, it can be assumed that the CF will be similar as well. To really quantify the CF for the setup with the sample chamber in air, a different standard (e.g. ethanol or glassy carbon) can be used [1,5]. Especially glassy carbon is well suited for in-house experiments as it has a higher scattering cross-section. A good quality measurement of glassy carbon only takes several minutes to few hours, compared to the rather long exposure for water ( $24 \mathrm{~h}$ ). However, the differential scattering cross-section is not known and needs to be experimentally determined [5] and unlike water has no flat part (Fig. A.2b). Therefore, the differential scattering cross-section needs to be resolved using a primary standard like water, or measured using small angle neutron scattering (SANS) [1]. Once the scattering cross-section is determined, the CF can be calculated using the same glassy carbon. Zhang et al. performed not only long-term studies over nine months but also radiation damage tests on glassy carbon. They could show that no significant difference between the signal within those nine months was observed as well as no radiation damage could be detected [3]. 


\section{References}

1. Fan, L., Degen, M., Bendle, S., Grupido, N. \& Ilavsky, J. The Absolute Calibration of a SmallAngle Scattering Instrument with a Laboratory X-ray Source. J. Phys.: Conf. Ser. 247, 012005 (2010).

2. Pauw, B. R. The Impossible Project"-How to do a perfect SAXS measurement in (2011).

3. Zhang, F. et al. Glassy Carbon as an Absolute Intensity Calibration Standard for SmallAngle Scattering. Metall. Mater. Trans. A 41, 1151-1158 (2009).

4. Orthaber, D., Bergmann, A. \& Glatter, O. SAXS experiments on absolute scale with Kratky systems using water as a secondary standard. J. Appl. Crystallogr. 33, 218-225 (2000).

5. Dreiss, C. A., Jack, K. S. \& Parker, A. P. On the absolute calibration of bench-top small-angle X-ray scattering instruments: a comparison of different standard methods. J. Appl. Crystallogr. 39, 32-38 (2006).

6. Small-Angle Scattering of X-Rays (eds Guinier, A. \& Fournet, G.) (John Wiley and Sonst. Inc., 1955).

7. Pedersen, J. S. Form factors of block copolymer micelles with spherical, ellipsoidal and cylindrical cores. J. Appl. Crystallogr. 33, 637-640 (2000).

8. Brennich, M. E. et al. Impact of ion valency on the assembly of vimentin studied by quantitative small angle X-ray scattering. Soft Matter 10, 2059-2068 (2014). 


\section{Appendix B}

\section{Model Fits}

All scattering profiles of vimentin assembled with different ions at several concentrations are shown here. The data are fit to a micelle model introduced by Pedersen [7] and extended by an additional term for tetrameric vimentin [8]. With this addition to the model, vimentin tetramers, which can be present in the solution and contribute to the scattering signal are included. Experiments are conducted 1 - 5 times for each ion and ion concentration. In case of multiple experiments for one condition, all scattering profiles are included to retrieve one fit curve. By fitting the model to the data, the individual contributions $\left(F_{c}, F_{s}, S_{s c}\right.$ and $\left.S_{c c}\right)$ can be retrieved. All model implementations, calculations and plots shown in the following are made by Manuel Marschall (Weierstraß Institute, Berlin, Germany). 


\section{B.1 Model Fits of Vimentin Assembled in the Presence of KCl}

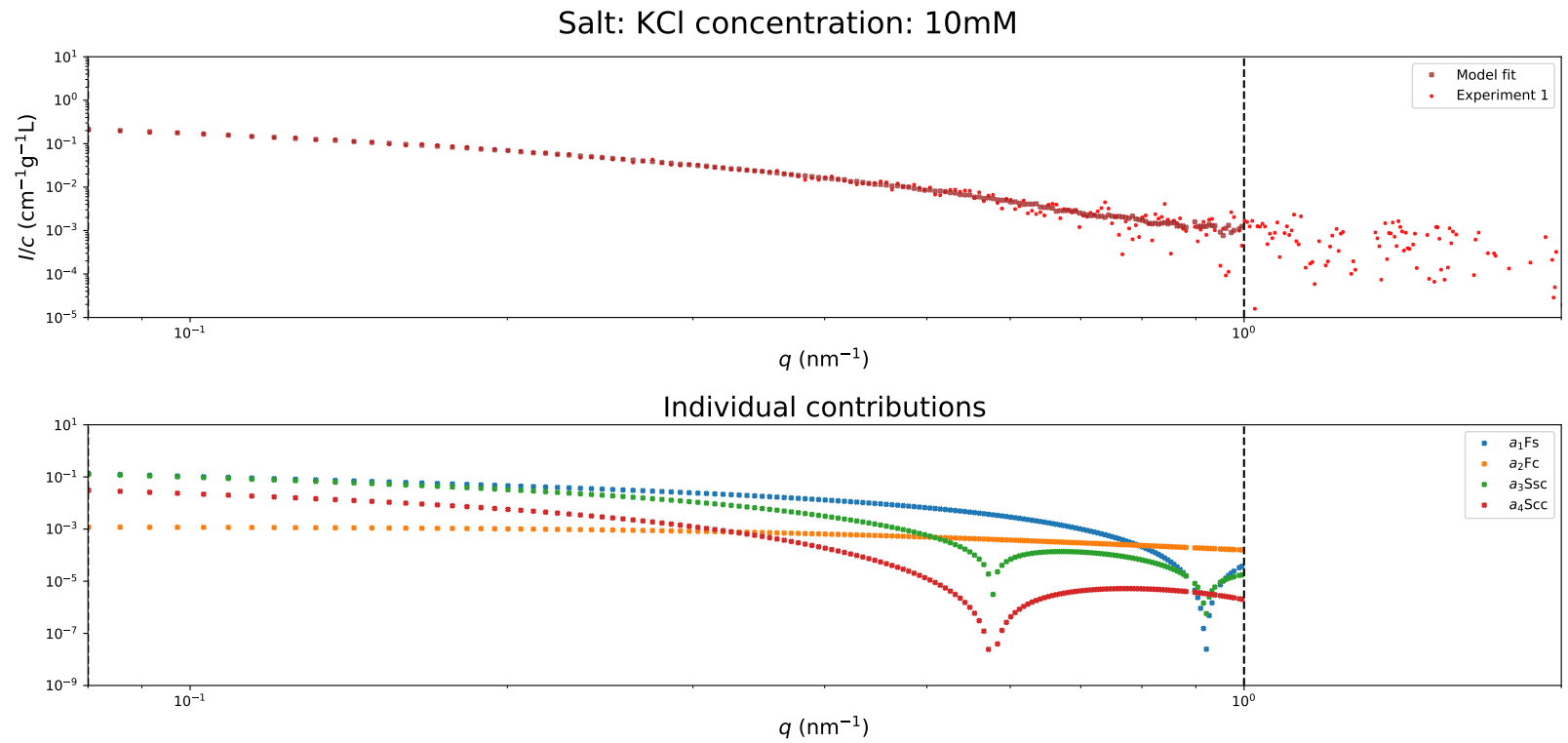

Results from the micelle model fitted to the data. In the upper image, all scattering profiles of the individual experiments plus the fit curve are shown. In the lower graphic, the individual contributions of the four terms $\left(F_{c}, F_{s}, S_{s c}\right.$ and $S_{c c}$ ) are displayed.

Salt: $\mathrm{KCl}$ concentration: $20 \mathrm{mM}$

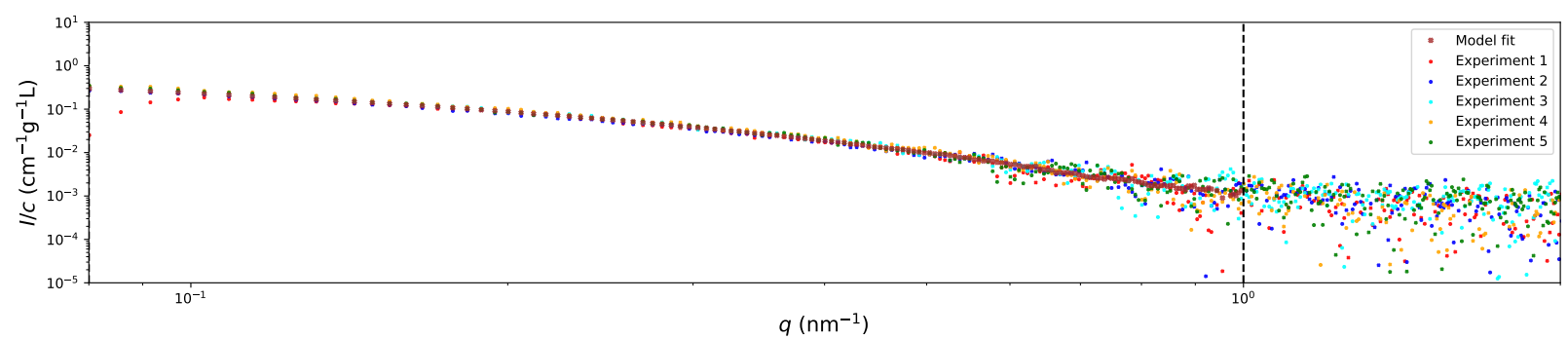

Individual contributions

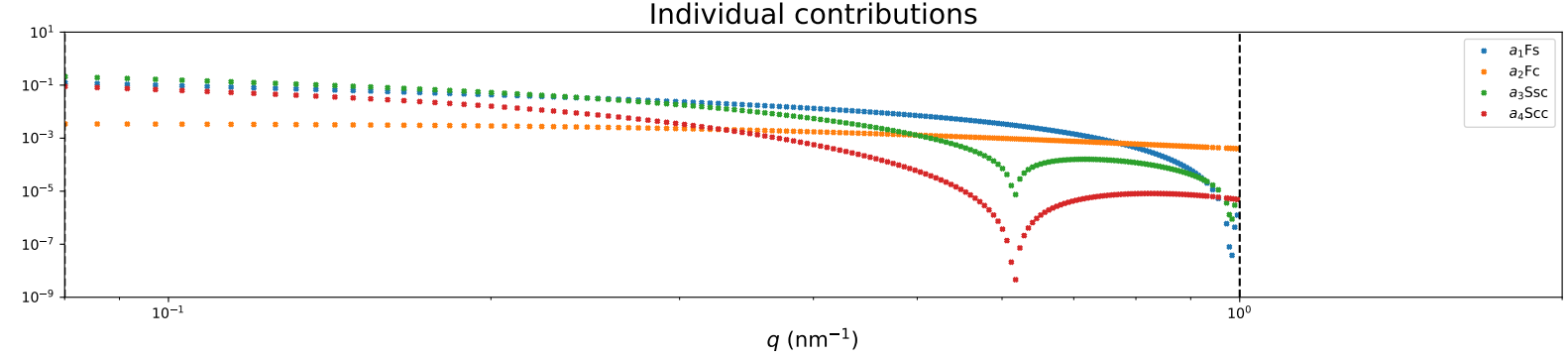

Results from the micelle model fitted to the data. In the upper image, all scattering profiles of the individual experiments plus the fit curve are shown. In the lower graphic, the individual contributions of the four terms $\left(F_{c}, F_{s}, S_{s c}\right.$ and $S_{c c}$ ) are displayed. 
Salt: $\mathrm{KCl}$ concentration: $30 \mathrm{mM}$

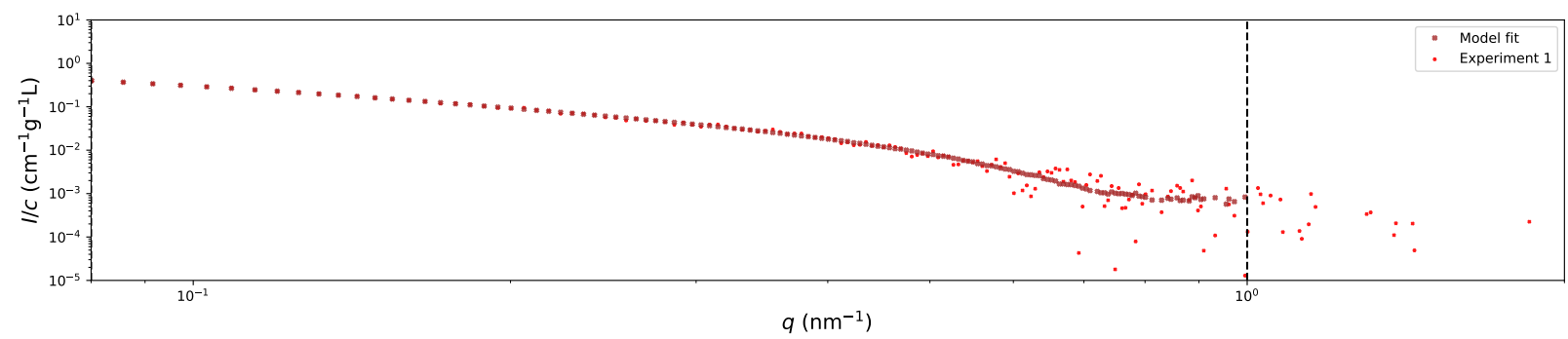

Individual contributions

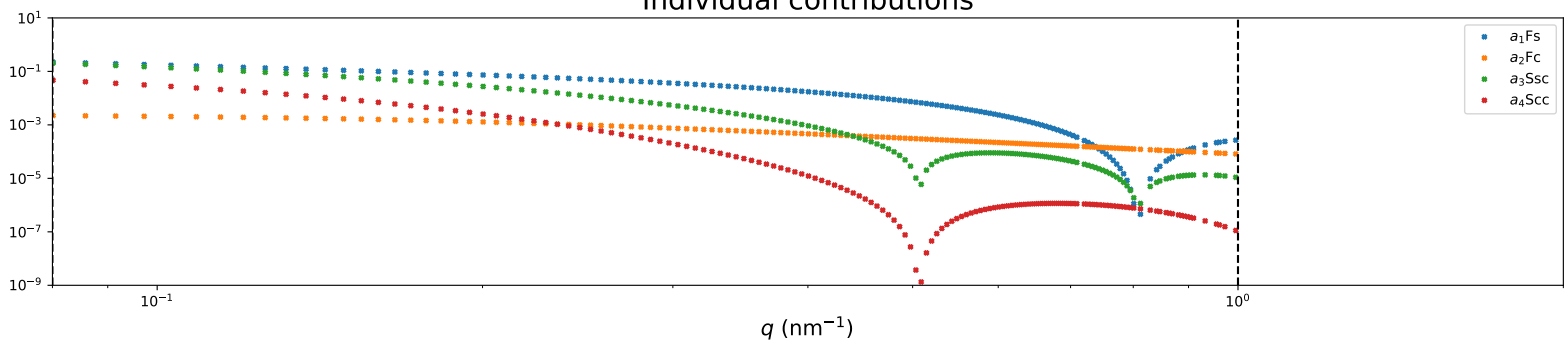

Results from the micelle model fitted to the data. In the upper image, all scattering profiles of the individual experiments plus the fit curve are shown. In the lower graphic, the individual contributions of the four terms $\left(F_{c}, F_{s}, S_{s c}\right.$ and $S_{c c}$ ) are displayed.

Salt: $\mathrm{KCl}$ concentration: $40 \mathrm{mM}$

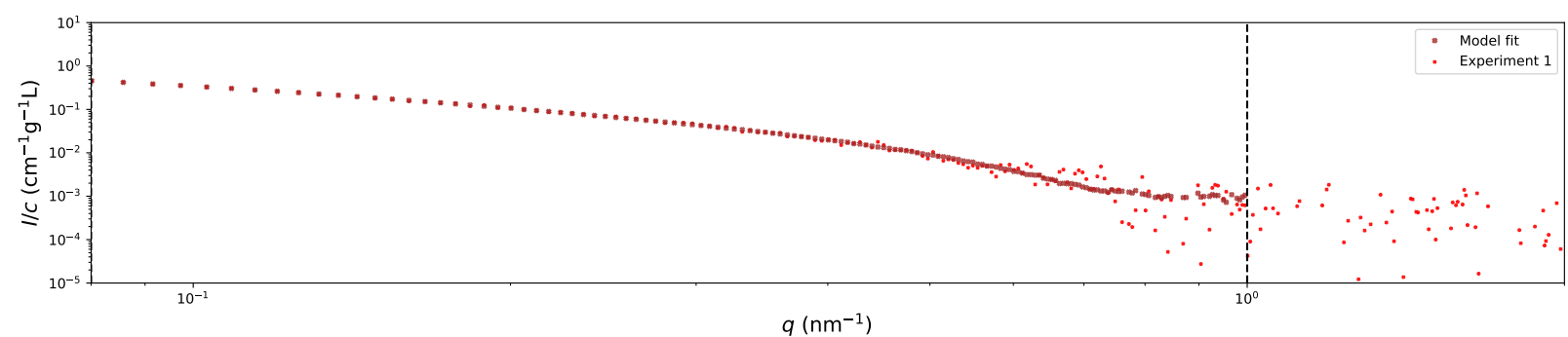

Individual contributions

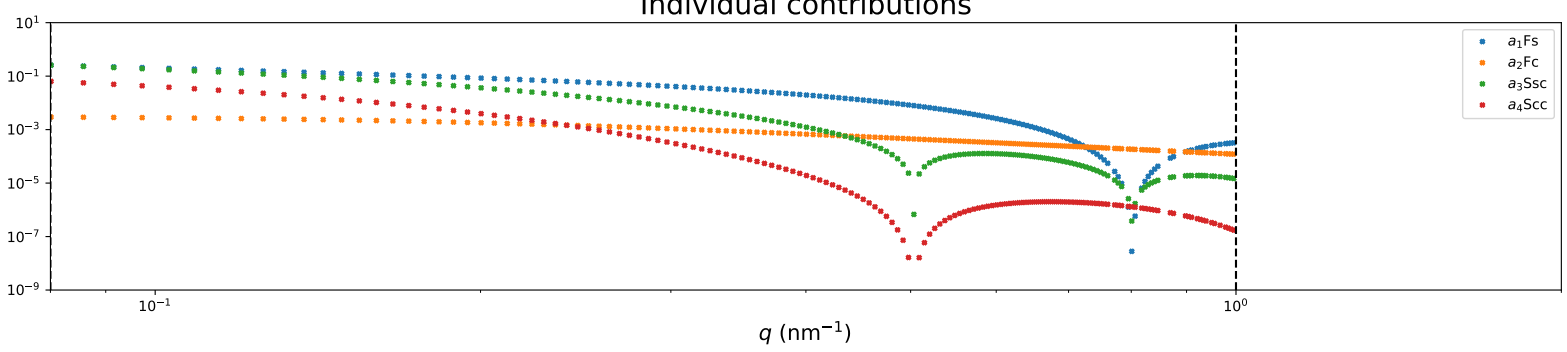

Results from the micelle model fitted to the data. In the upper image, all scattering profiles of the individual experiments plus the fit curve are shown. In the lower graphic, the individual contributions of the four terms $\left(F_{c}, F_{s}, S_{s c}\right.$ and $S_{c c}$ ) are displayed. 
Salt: $\mathrm{KCl}$ concentration: $50 \mathrm{mM}$

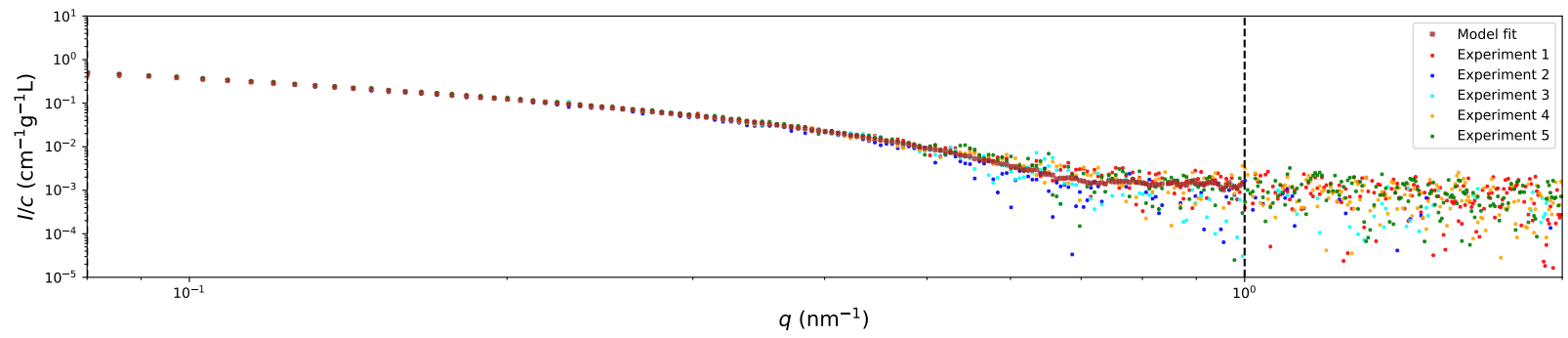

Individual contributions

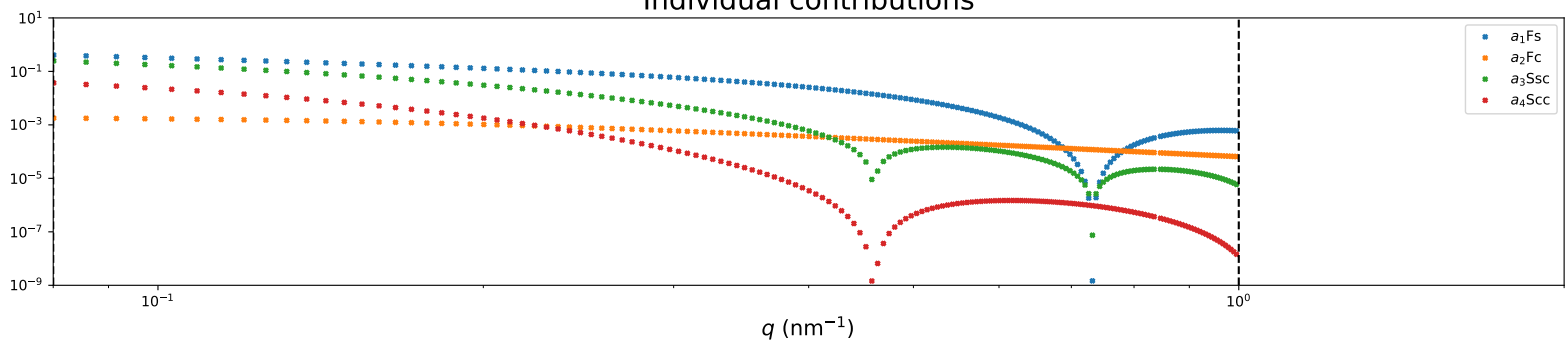

Results from the micelle model fitted to the data. In the upper image, all scattering profiles of the individual experiments plus the fit curve are shown. In the lower graphic, the individual contributions of the four terms $\left(F_{c}, F_{s}, S_{s c}\right.$ and $S_{c c}$ ) are displayed.

Salt: $\mathrm{KCl}$ concentration: $80 \mathrm{mM}$

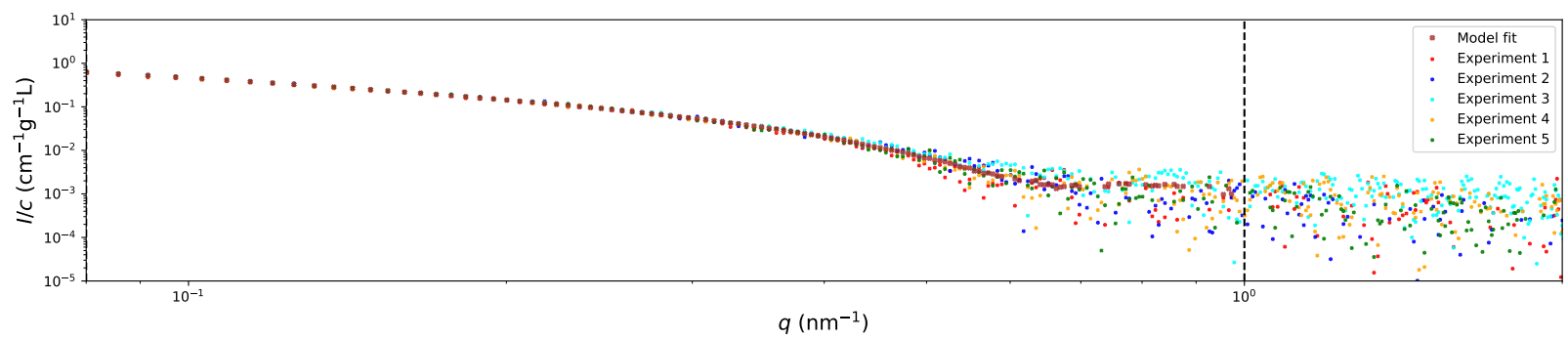

Individual contributions

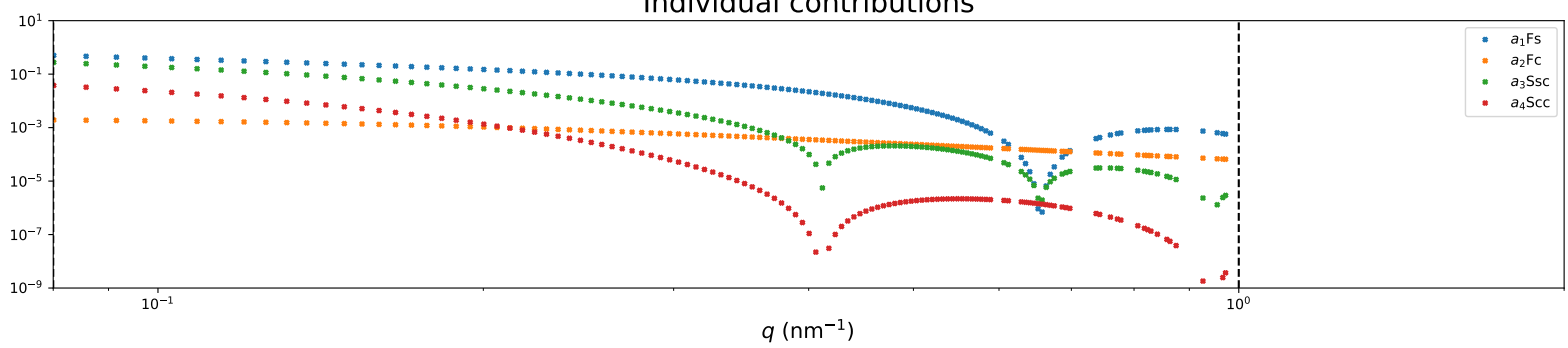

Results from the micelle model fitted to the data. In the upper image, all scattering profiles of the individual experiments plus the fit curve are shown. In the lower graphic, the individual contributions of the four terms $\left(F_{c}, F_{s}, S_{s c}\right.$ and $S_{c c}$ ) are displayed. 
Salt: $\mathrm{KCl}$ concentration: $100 \mathrm{mM}$

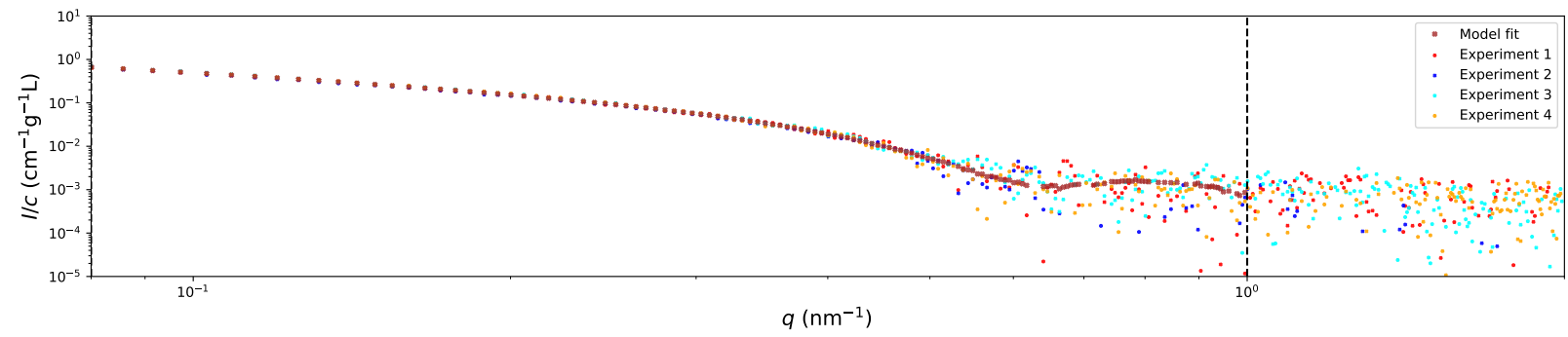

Individual contributions

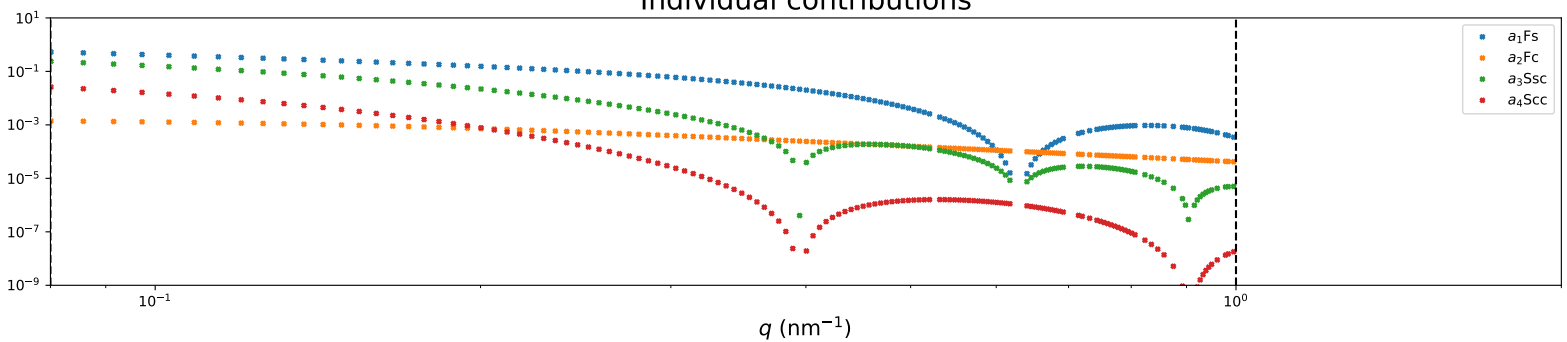

Results from the micelle model fitted to the data. In the upper image, all scattering profiles of the individual experiments plus the fit curve are shown. In the lower graphic, the individual contributions of the four terms $\left(F_{c}, F_{s}, S_{s c}\right.$ and $S_{c c}$ ) are displayed.

Salt: $\mathrm{KCl}$ concentration: $150 \mathrm{mM}$

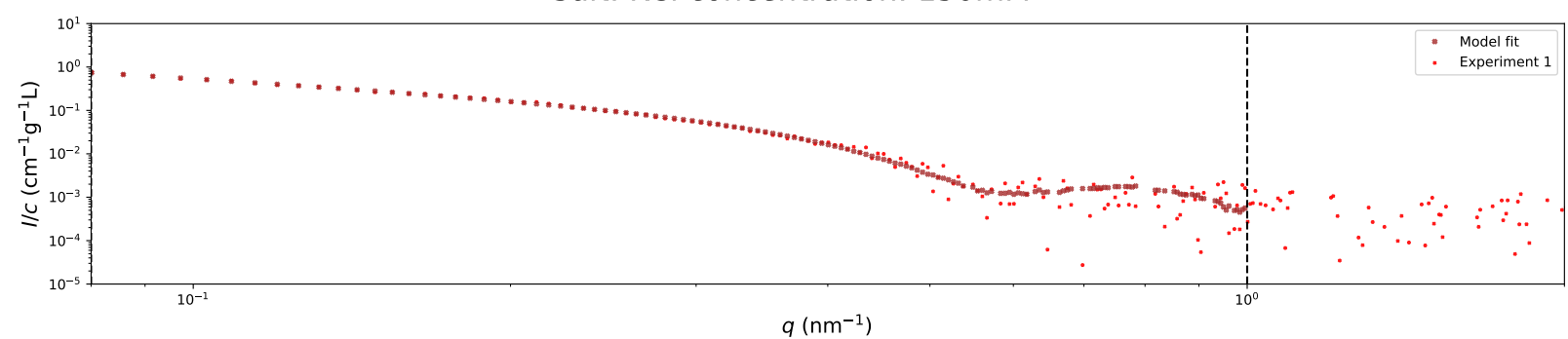

Individual contributions

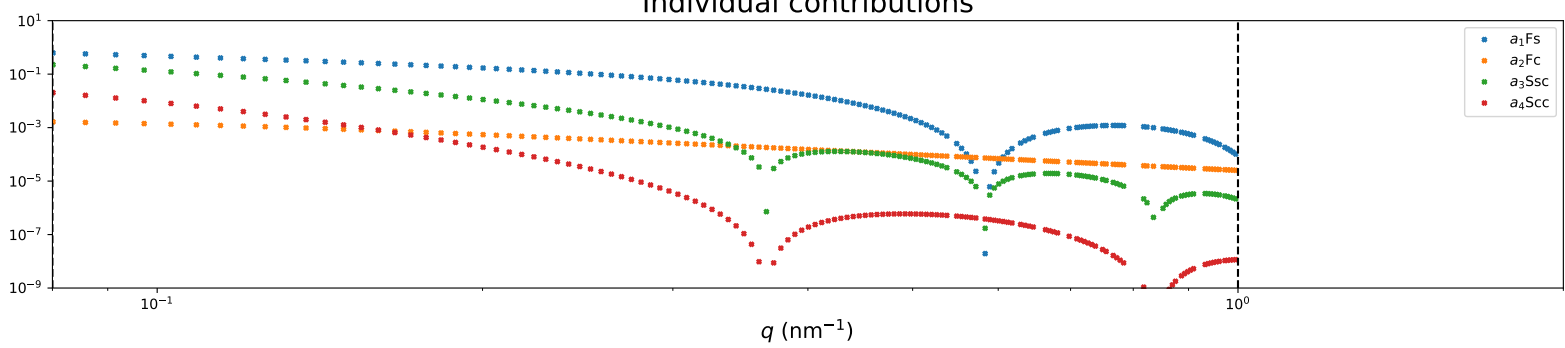

Results from the micelle model fitted to the data. In the upper image, all scattering profiles of the individual experiments plus the fit curve are shown. In the lower graphic, the individual contributions of the four terms $\left(F_{c}, F_{s}, S_{s c}\right.$ and $S_{c c}$ ) are displayed. 


\section{B.2 Model Fits of Vimentin Assembled in the Presence of $\mathrm{NaCl}$}

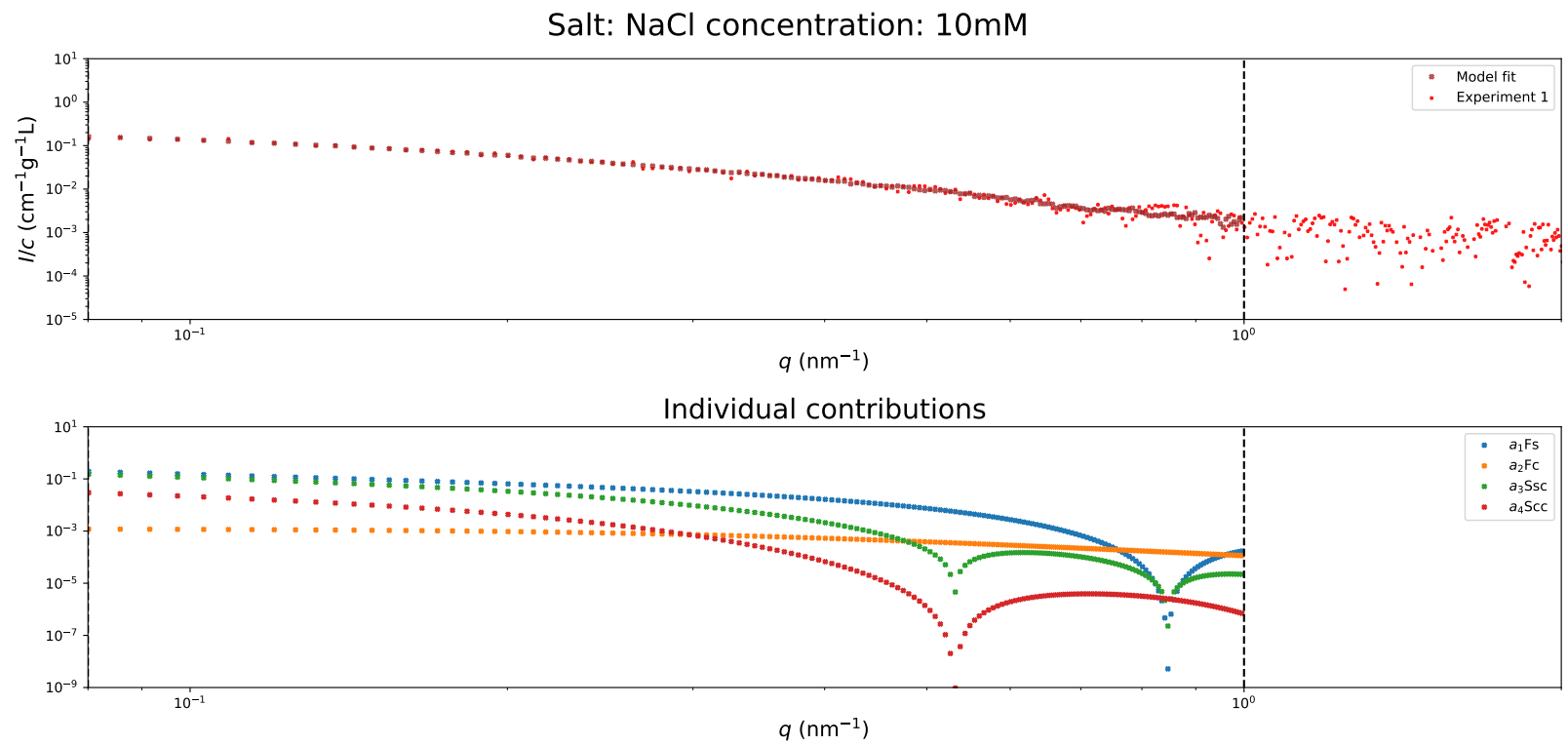

Results from the micelle model fitted to the data. In the upper image, all scattering profiles of the individual experiments plus the fit curve are shown. In the lower graphic, the individual contributions of the four terms $\left(F_{c}, F_{s}, S_{s c}\right.$ and $S_{c c}$ ) are displayed.

Salt: $\mathrm{NaCl}$ concentration: $20 \mathrm{mM}$

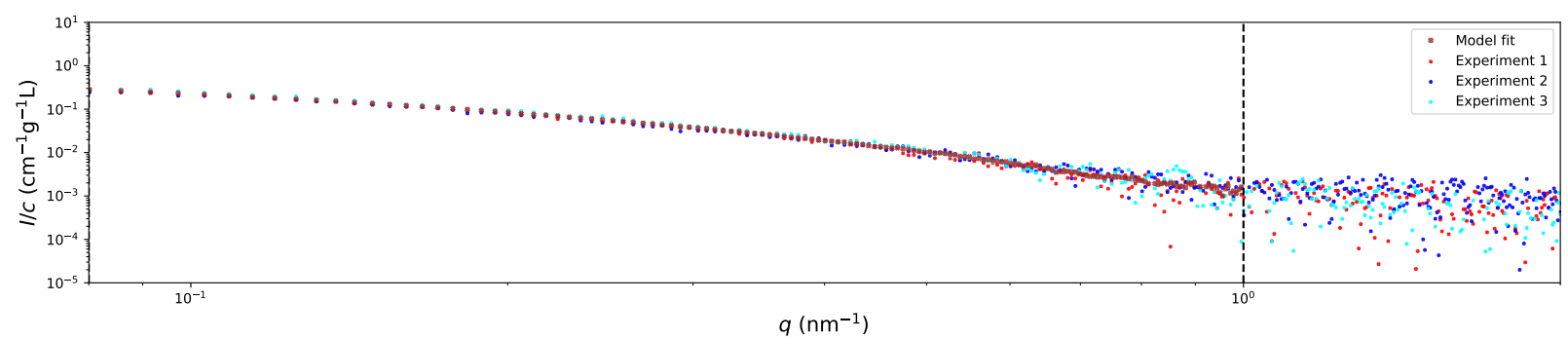

Individual contributions

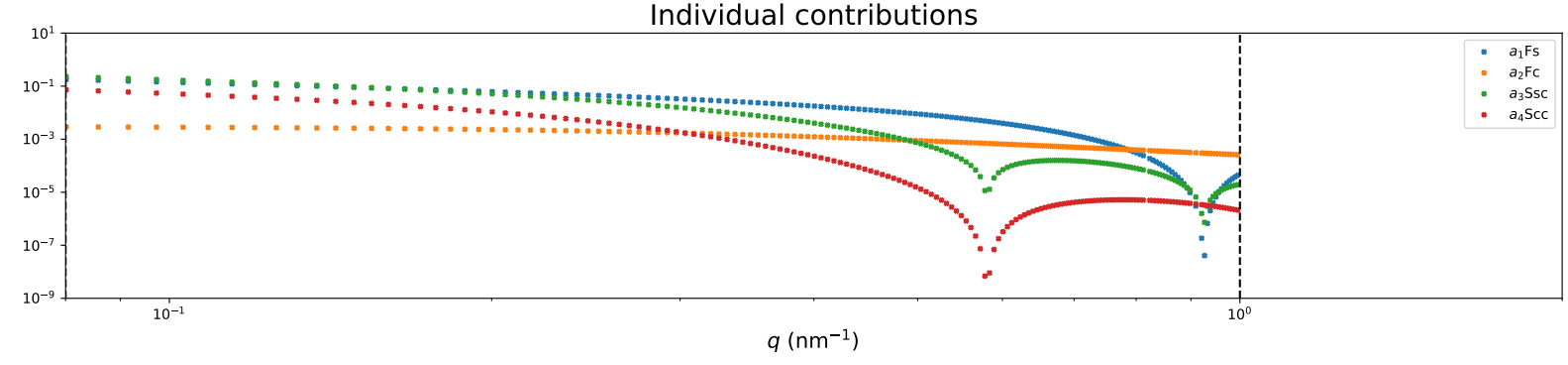

Results from the micelle model fitted to the data. In the upper image, all scattering profiles of the individual experiments plus the fit curve are shown. In the lower graphic, the individual contributions of the four terms $\left(F_{c}, F_{s}, S_{s c}\right.$ and $S_{c c}$ ) are displayed. 
Salt: $\mathrm{NaCl}$ concentration: $30 \mathrm{mM}$

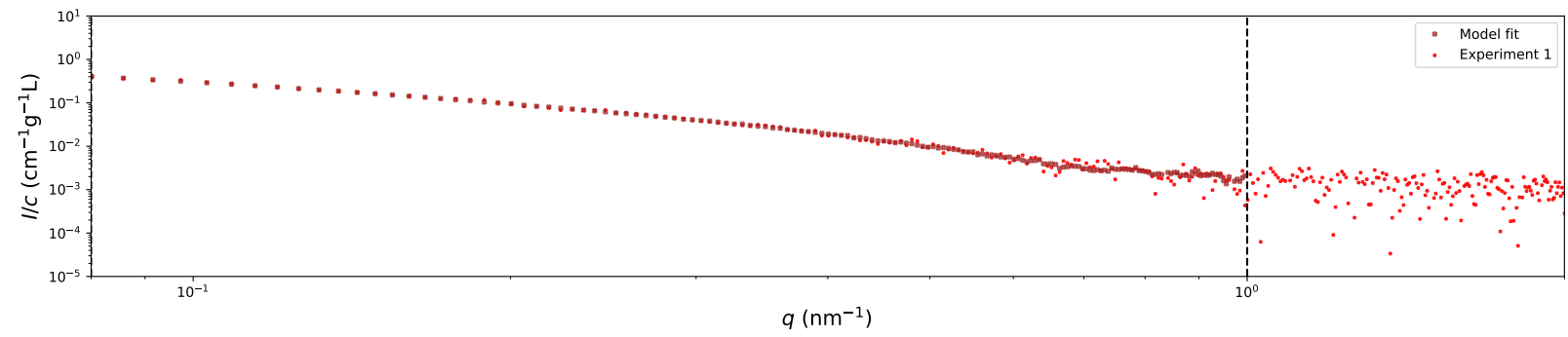

Individual contributions

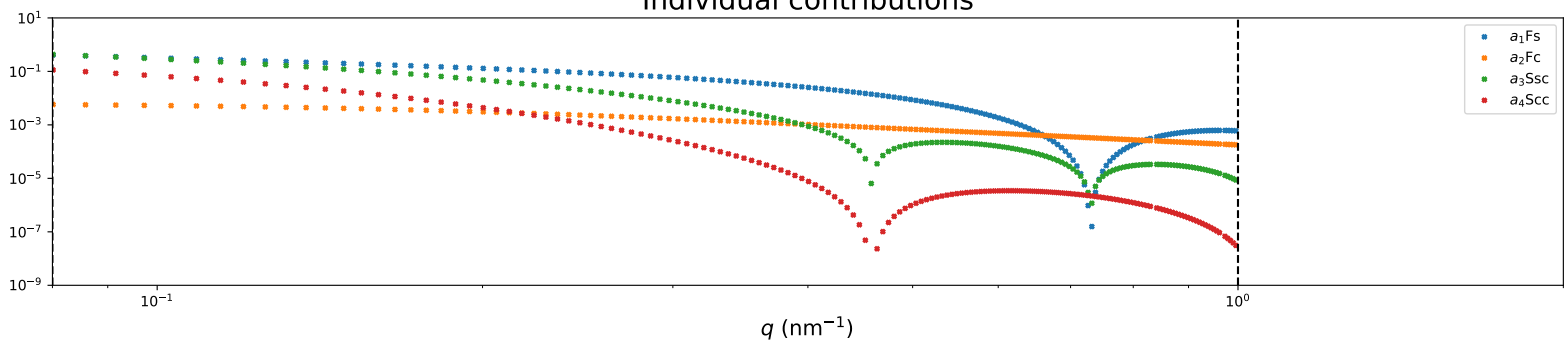

Results from the micelle model fitted to the data. In the upper image, all scattering profiles of the individual experiments plus the fit curve are shown. In the lower graphic, the individual contributions of the four terms $\left(F_{c}, F_{s}, S_{s c}\right.$ and $S_{c c}$ ) are displayed.

Salt: $\mathrm{NaCl}$ concentration: $40 \mathrm{mM}$

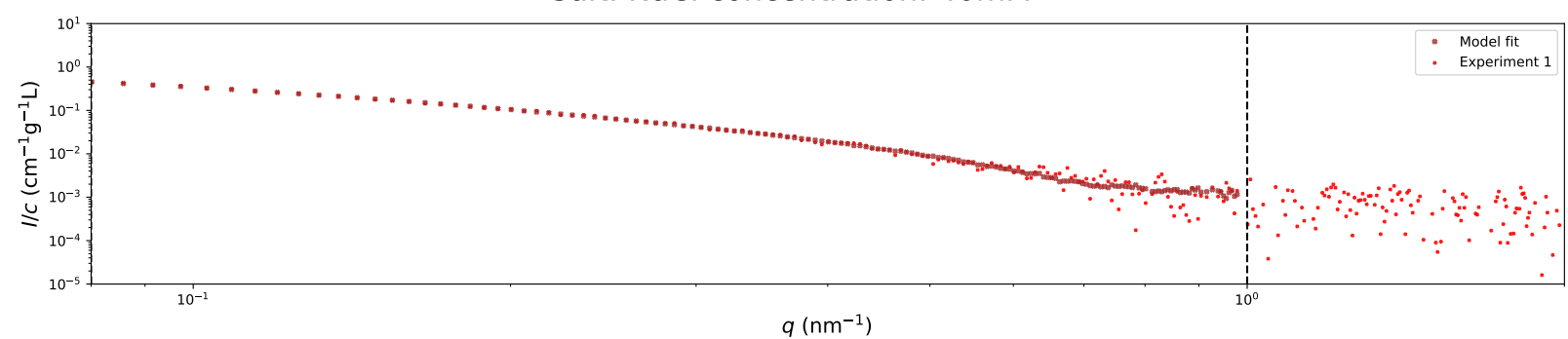

Individual contributions

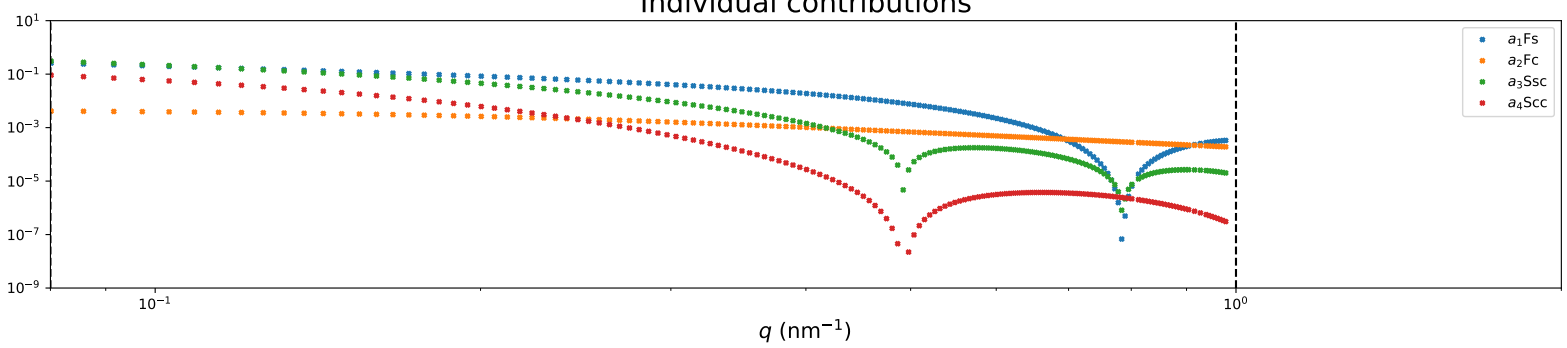

Results from the micelle model fitted to the data. In the upper image, all scattering profiles of the individual experiments plus the fit curve are shown. In the lower graphic, the individual contributions of the four terms $\left(F_{c}, F_{s}, S_{s c}\right.$ and $S_{c c}$ ) are displayed. 
Salt: $\mathrm{NaCl}$ concentration: $50 \mathrm{mM}$

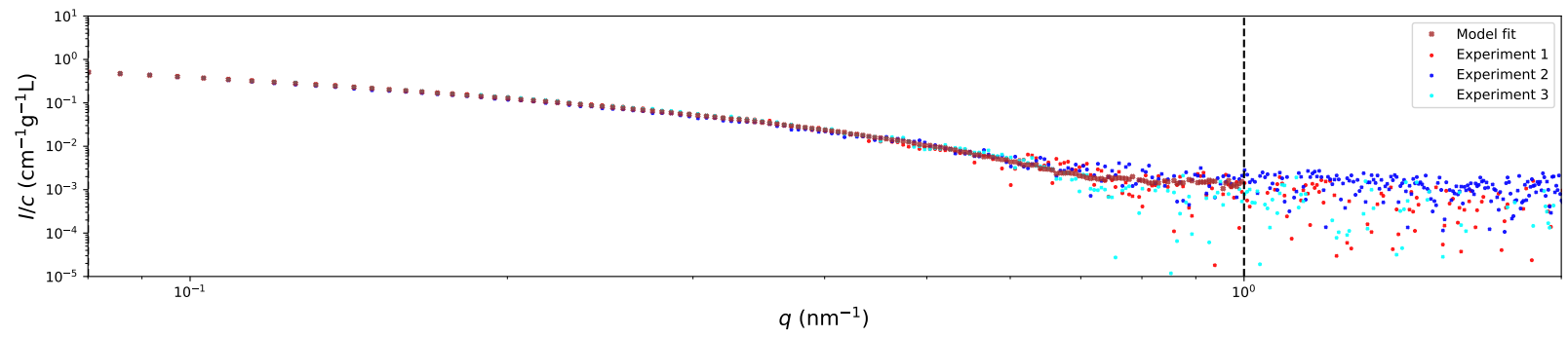

Individual contributions

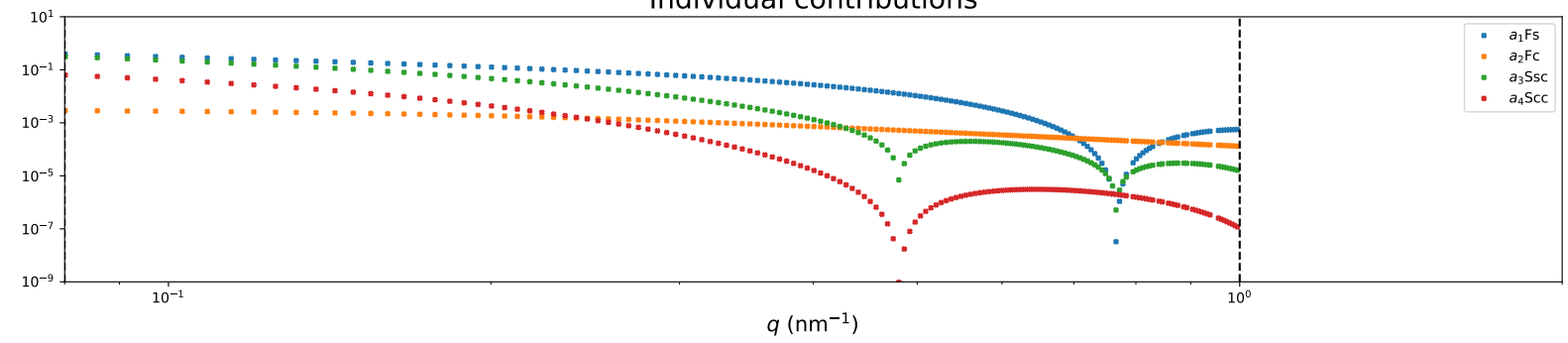

Results from the micelle model fitted to the data. In the upper image, all scattering profiles of the individual experiments plus the fit curve are shown. In the lower graphic, the individual contributions of the four terms $\left(F_{c}, F_{s}, S_{s c}\right.$ and $S_{c c}$ ) are displayed.

Salt: $\mathrm{NaCl}$ concentration: $80 \mathrm{mM}$

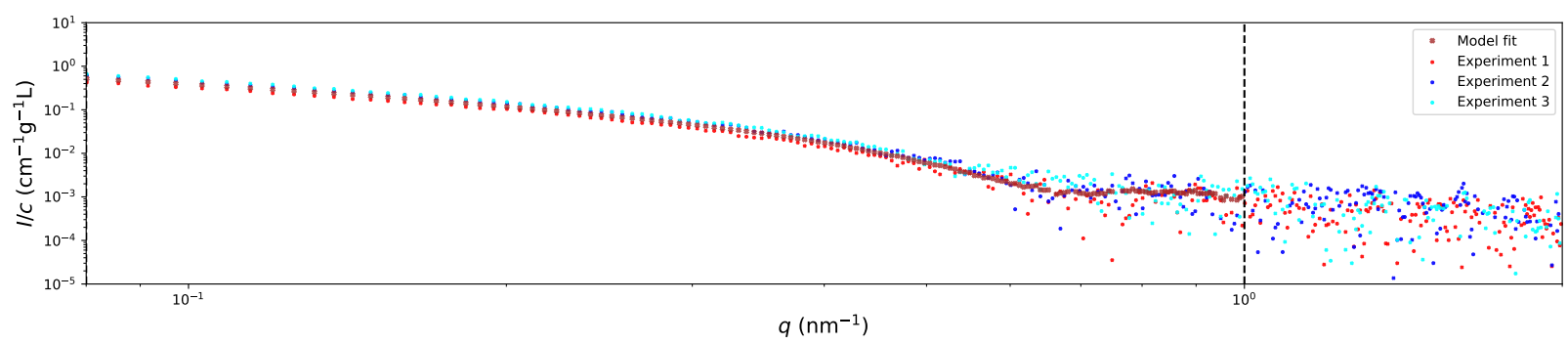

Individual contributions

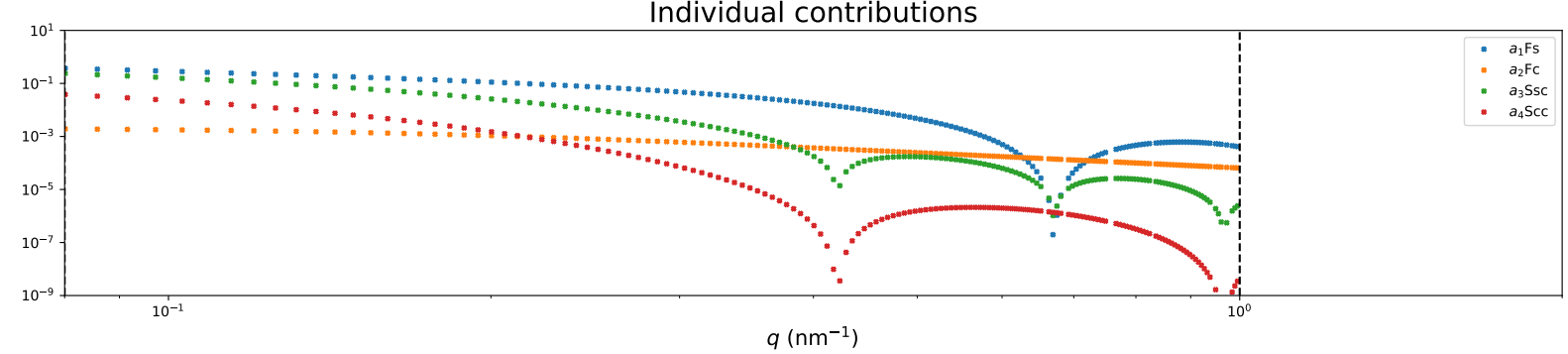

Results from the micelle model fitted to the data. In the upper image, all scattering profiles of the individual experiments plus the fit curve are shown. In the lower graphic, the individual contributions of the four terms $\left(F_{c}, F_{s}, S_{s c}\right.$ and $S_{c c}$ ) are displayed. 
Salt: $\mathrm{NaCl}$ concentration: $100 \mathrm{mM}$

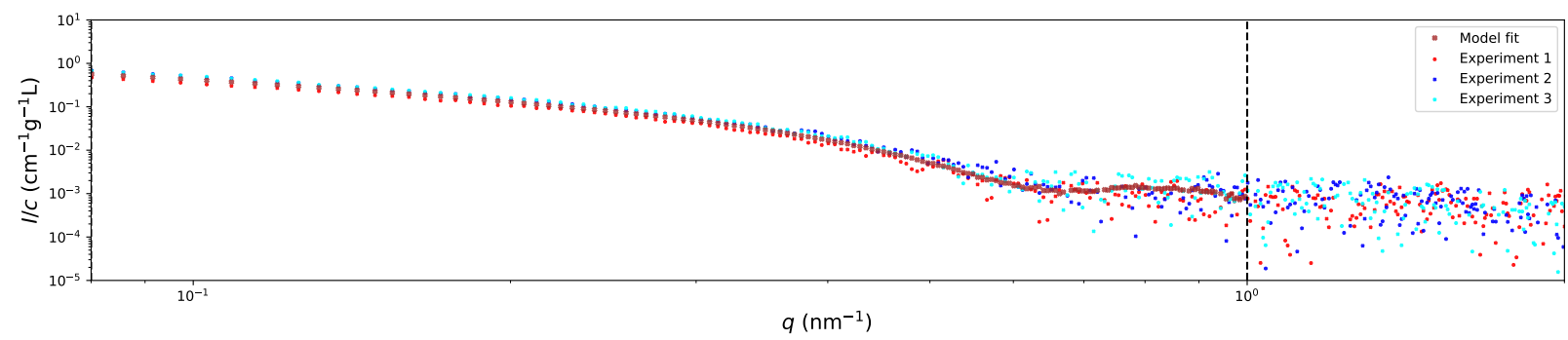

Individual contributions

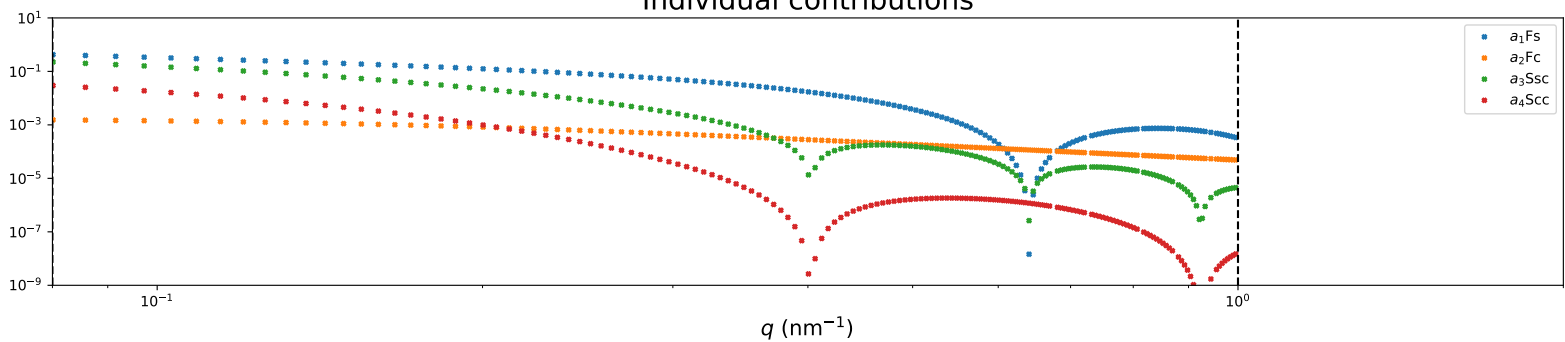

Results from the micelle model fitted to the data. In the upper image, all scattering profiles of the individual experiments plus the fit curve are shown. In the lower graphic, the individual contributions of the four terms $\left(F_{c}, F_{s}, S_{s c}\right.$ and $S_{c c}$ ) are displayed.

Salt: $\mathrm{NaCl}$ concentration: $150 \mathrm{mM}$

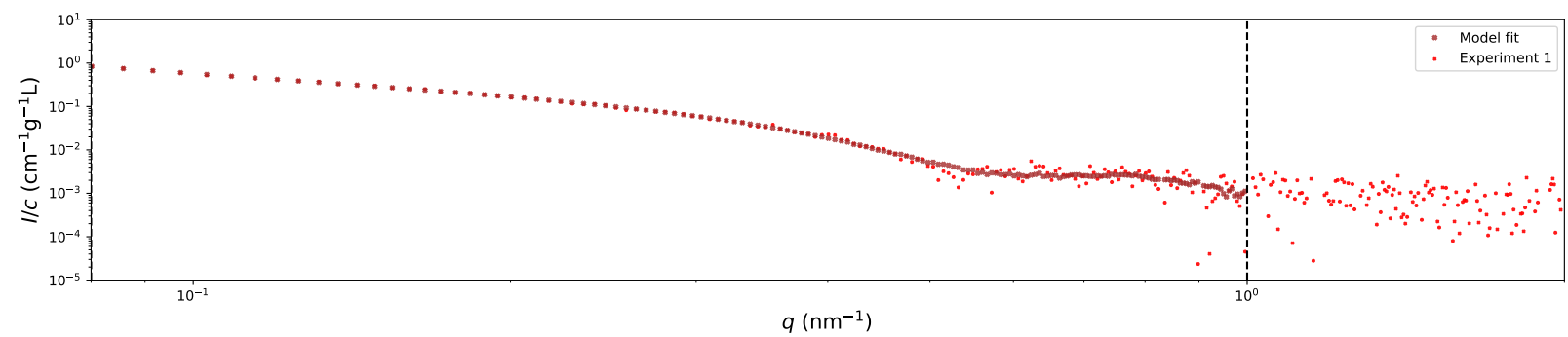

Individual contributions

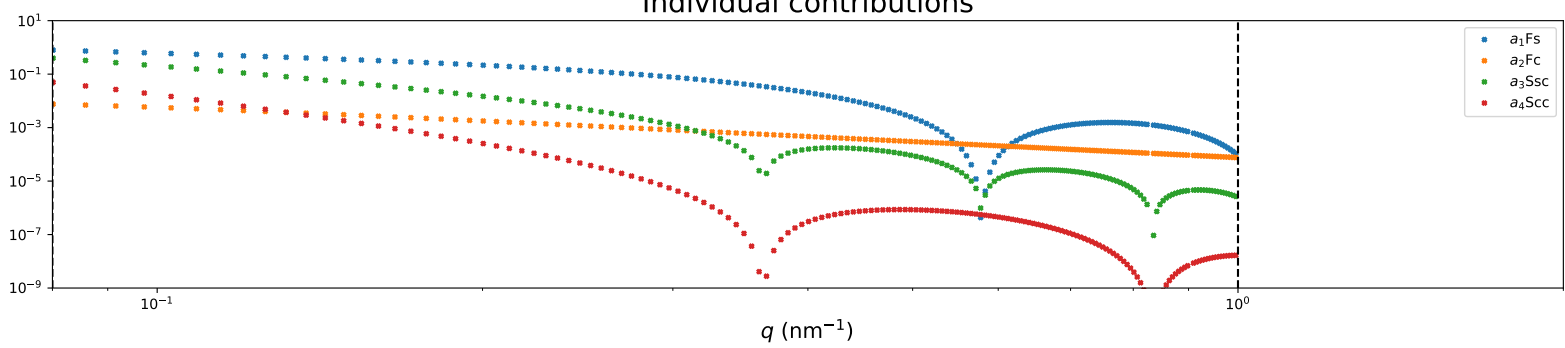

Results from the micelle model fitted to the data. In the upper image, all scattering profiles of the individual experiments plus the fit curve are shown. In the lower graphic, the individual contributions of the four terms $\left(F_{c}, F_{s}, S_{s c}\right.$ and $S_{c c}$ ) are displayed. 


\section{B.3 Model Fits of Vimentin Assembled in the Presence of $\mathbf{M g C l}_{2}$}

Salt: $\mathrm{MgCl}_{2}$ concentration: $0.5 \mathrm{mM}$

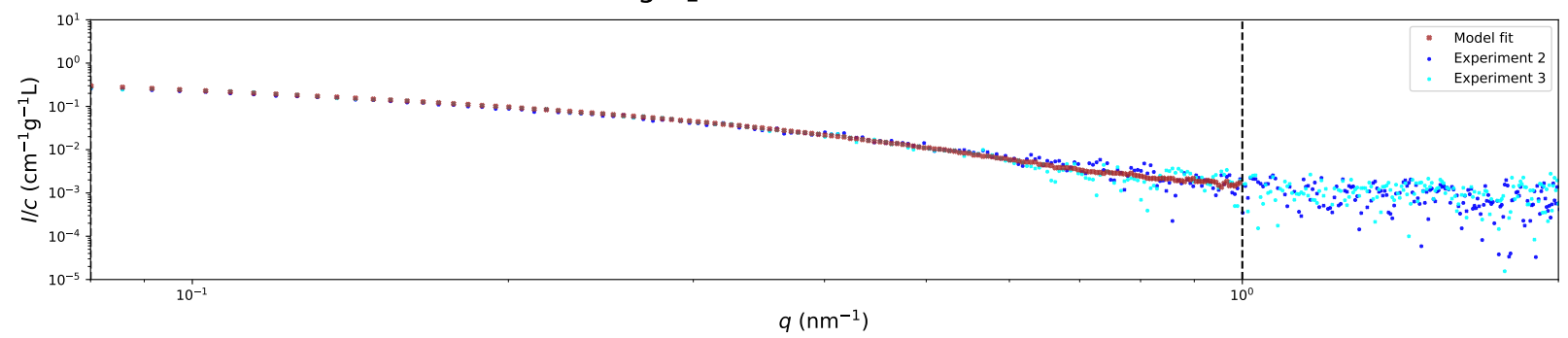

Individual contributions

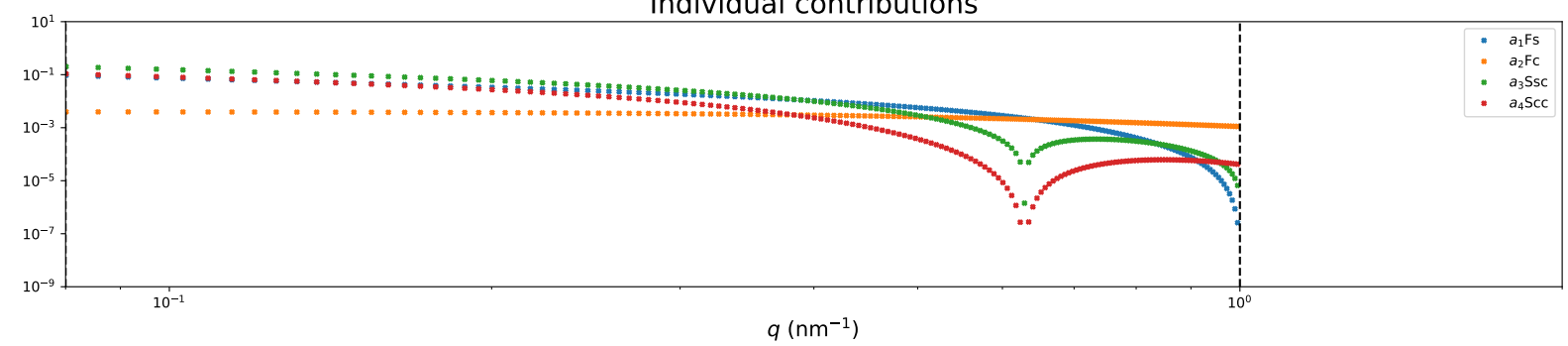

Results from the micelle model fitted to the data. In the upper image, all scattering profiles of the individual experiments plus the fit curve are shown. In the lower graphic, the individual contributions of the four terms $\left(F_{c}, F_{s}, S_{s c}\right.$ and $S_{c c}$ ) are displayed.

Salt: $\mathrm{MgCl}_{2}$ concentration: $1.0 \mathrm{mM}$

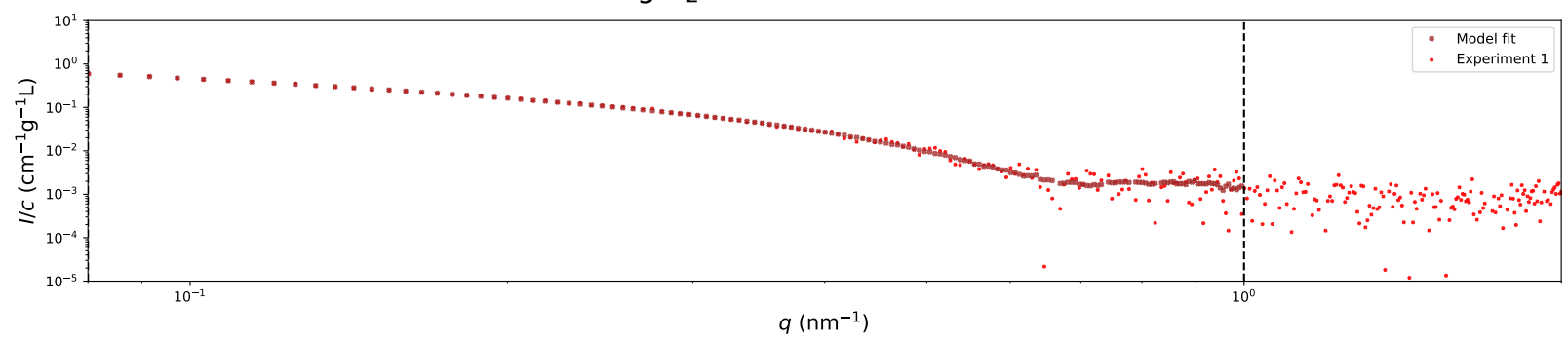

Individual contributions

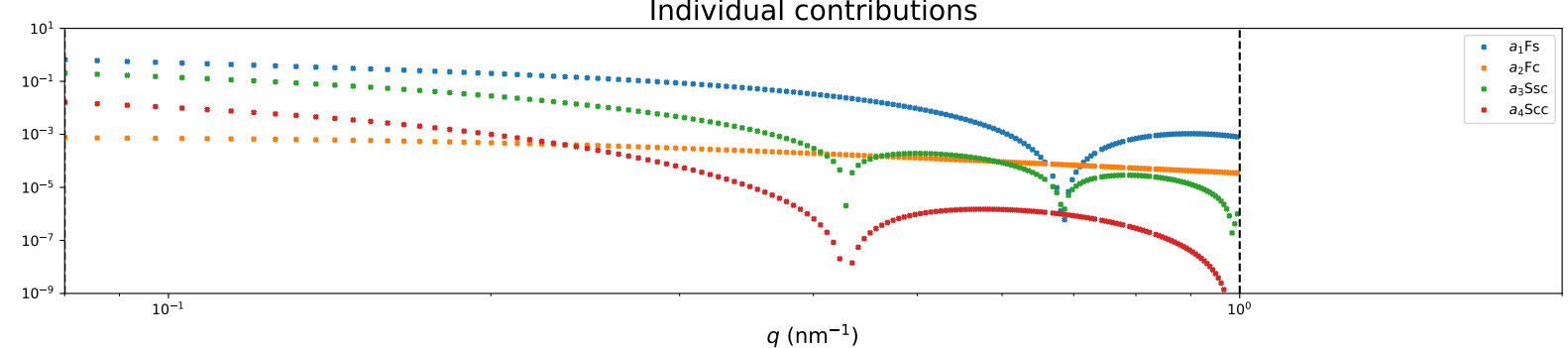

Results from the micelle model fitted to the data. In the upper image, all scattering profiles of the individual experiments plus the fit curve are shown. In the lower graphic, the individual contributions of the four terms $\left(F_{c}, F_{s}, S_{s c}\right.$ and $S_{c c}$ ) are displayed. 
Salt: $\mathrm{MgCl}_{2}$ concentration: $1.5 \mathrm{mM}$

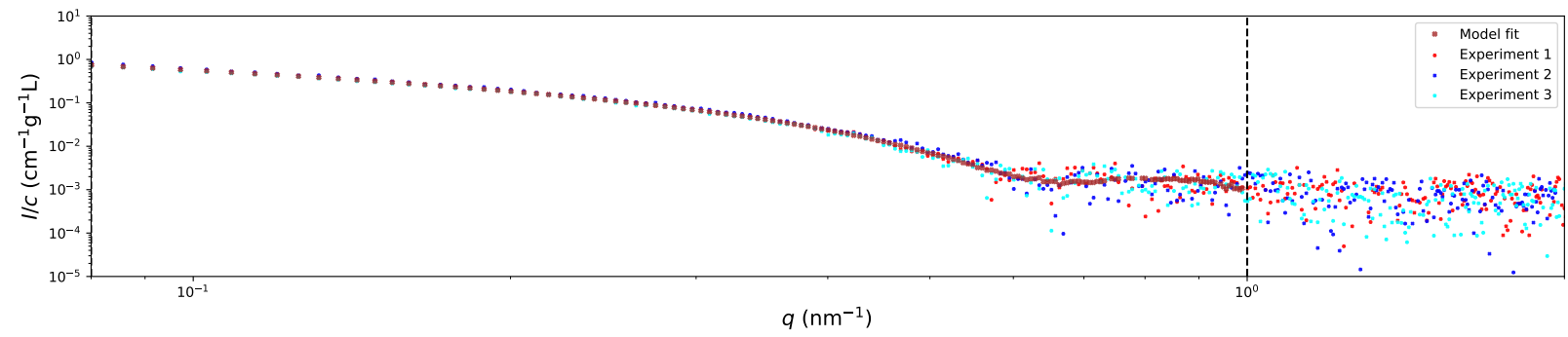

Individual contributions

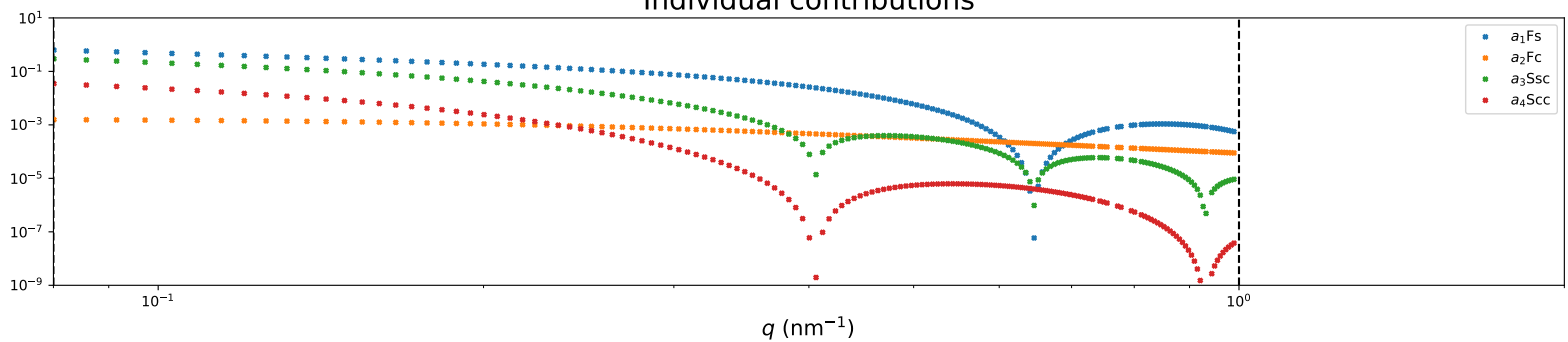

Results from the micelle model fitted to the data. In the upper image, all scattering profiles of the individual experiments plus the fit curve are shown. In the lower graphic, the individual contributions of the four terms $\left(F_{c}, F_{s}, S_{s c}\right.$ and $S_{c c}$ ) are displayed.

Salt: $\mathrm{MgCl}_{2}$ concentration: $2.0 \mathrm{mM}$

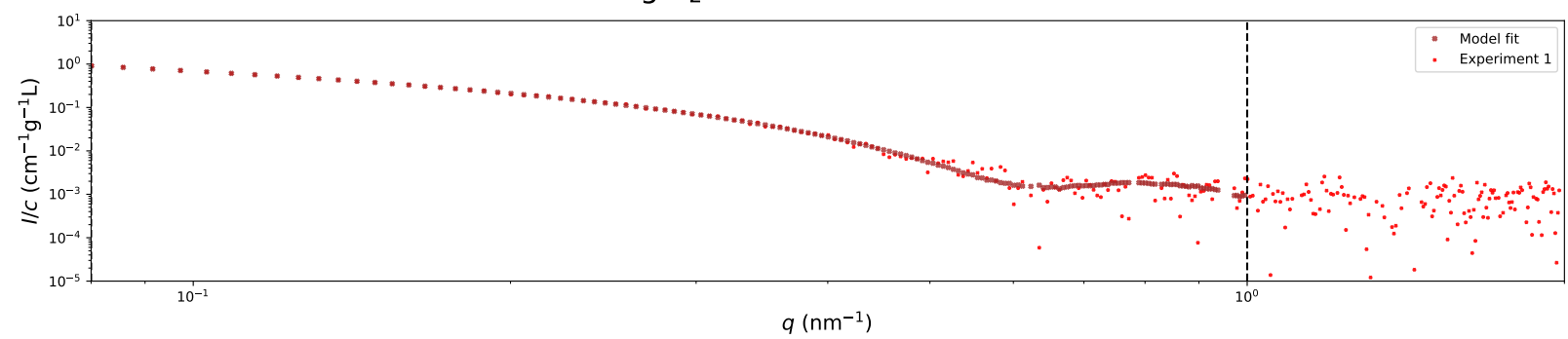

Individual contributions

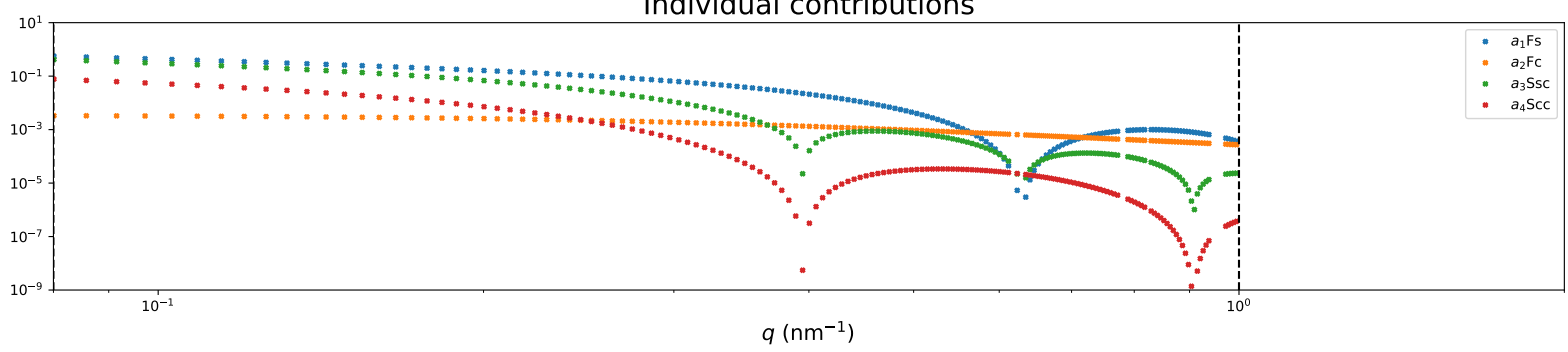

Results from the micelle model fitted to the data. In the upper image, all scattering profiles of the individual experiments plus the fit curve are shown. In the lower graphic, the individual contributions of the four terms $\left(F_{c}, F_{s}, S_{s c}\right.$ and $S_{c c}$ ) are displayed. 
Salt: $\mathrm{MgCl}_{2}$ concentration: $2.5 \mathrm{mM}$

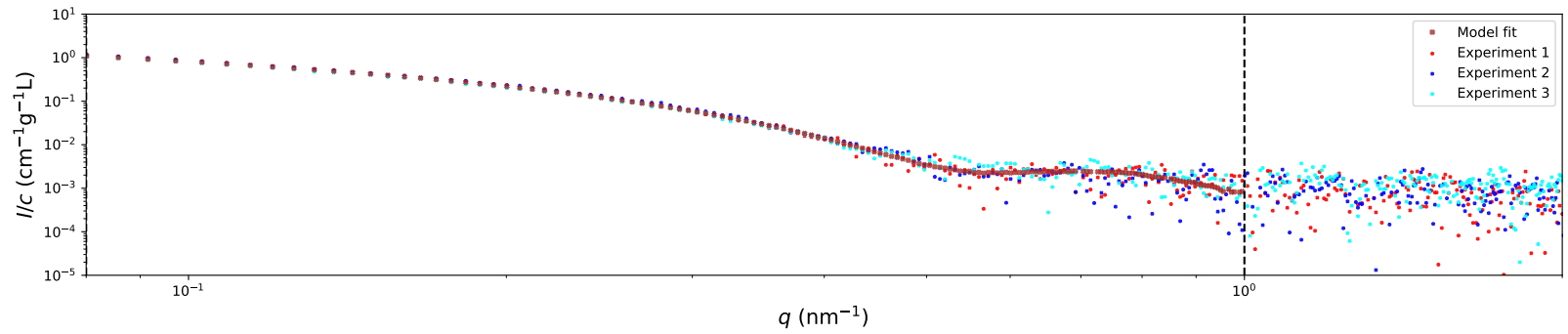

Individual contributions

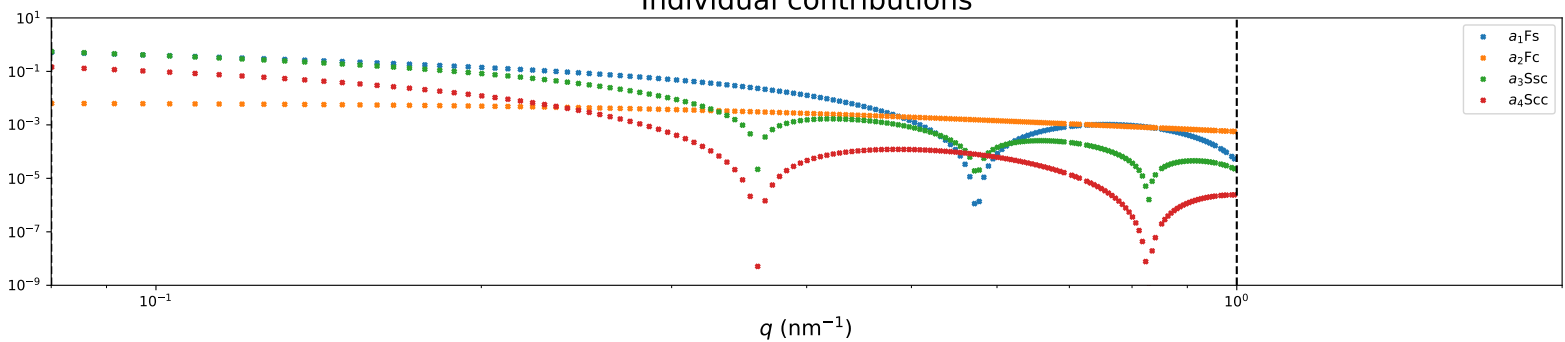

Results from the micelle model fitted to the data. In the upper image, all scattering profiles of the individual experiments plus the fit curve are shown. In the lower graphic, the individual contributions of the four terms $\left(F_{c}, F_{s}, S_{s c}\right.$ and $S_{c c}$ ) are displayed.

Salt: $\mathrm{MgCl}_{2}$ concentration: $4 \mathrm{mM}$
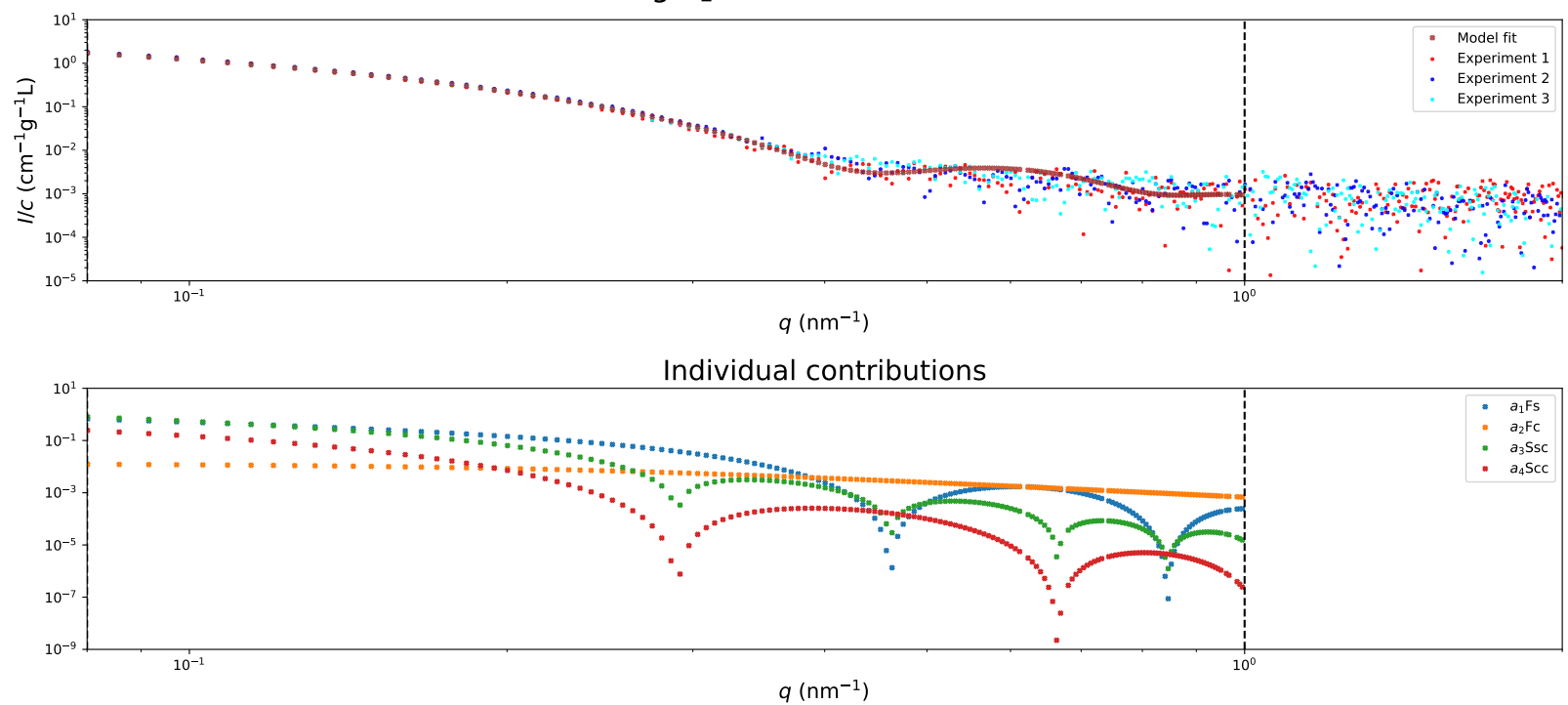

Results from the micelle model fitted to the data. In the upper image, all scattering profiles of the individual experiments plus the fit curve are shown. In the lower graphic, the individual contributions of the four terms $\left(F_{c}, F_{s}, S_{s c}\right.$ and $S_{c c}$ ) are displayed. 
Salt: $\mathrm{MgCl}_{2}$ concentration: $5 \mathrm{mM}$

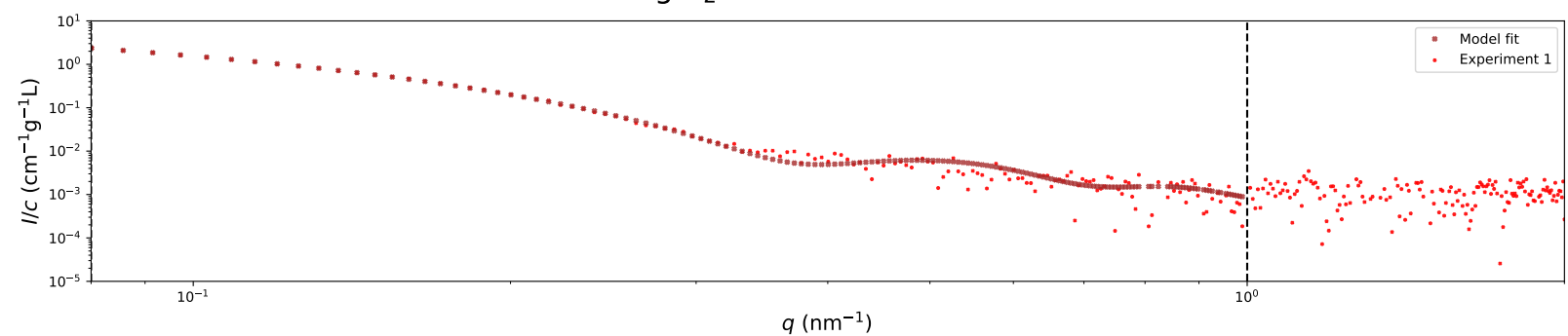

Individual contributions

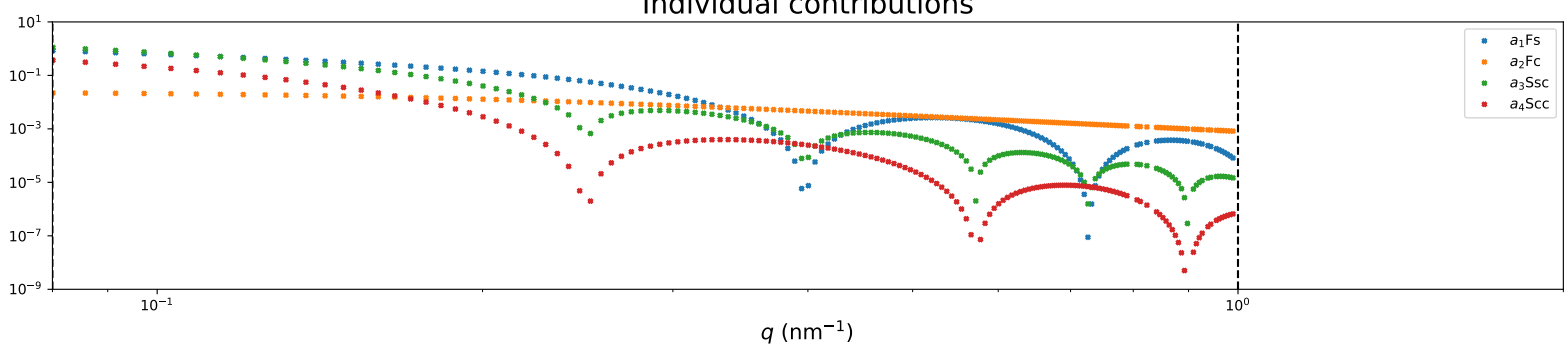

Results from the micelle model fitted to the data. In the upper image, all scattering profiles of the individual experiments plus the fit curve are shown. In the lower graphic, the individual contributions of the four terms $\left(F_{c}, F_{s}, S_{s c}\right.$ and $S_{c c}$ ) are displayed.

Salt: $\mathrm{MgCl}_{2}$ concentration: $10 \mathrm{mM}$

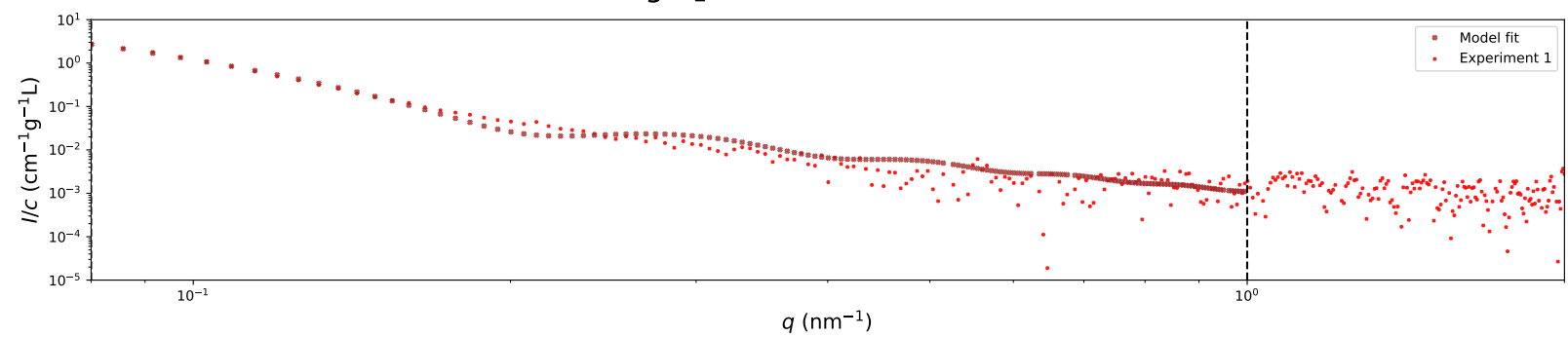

Individual contributions

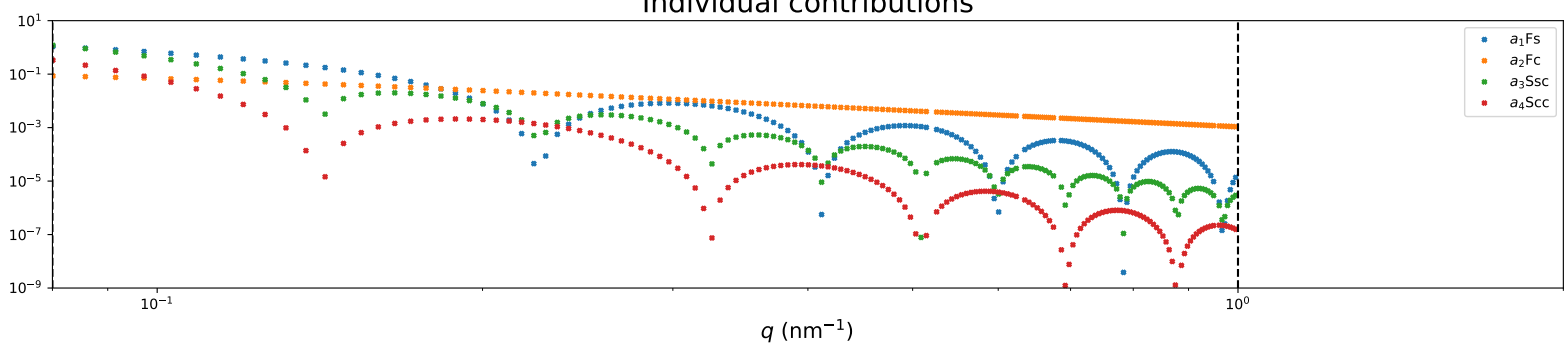

Results from the micelle model fitted to the data. In the upper image, all scattering profiles of the individual experiments plus the fit curve are shown. In the lower graphic, the individual contributions of the four terms $\left(F_{c}, F_{s}, S_{s c}\right.$ and $S_{c c}$ ) are displayed. 


\section{B.4 Model Fits of Vimentin Assembled in the Presence of $\mathrm{CaCl}_{2}$}

Salt: $\mathrm{CaCl}_{2}$ concentration: $0.5 \mathrm{mM}$

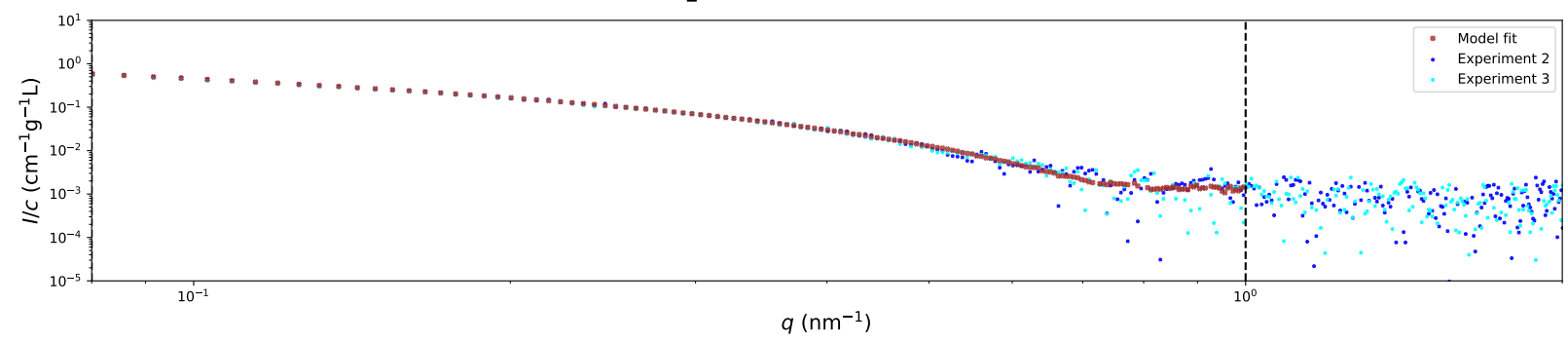

Individual contributions

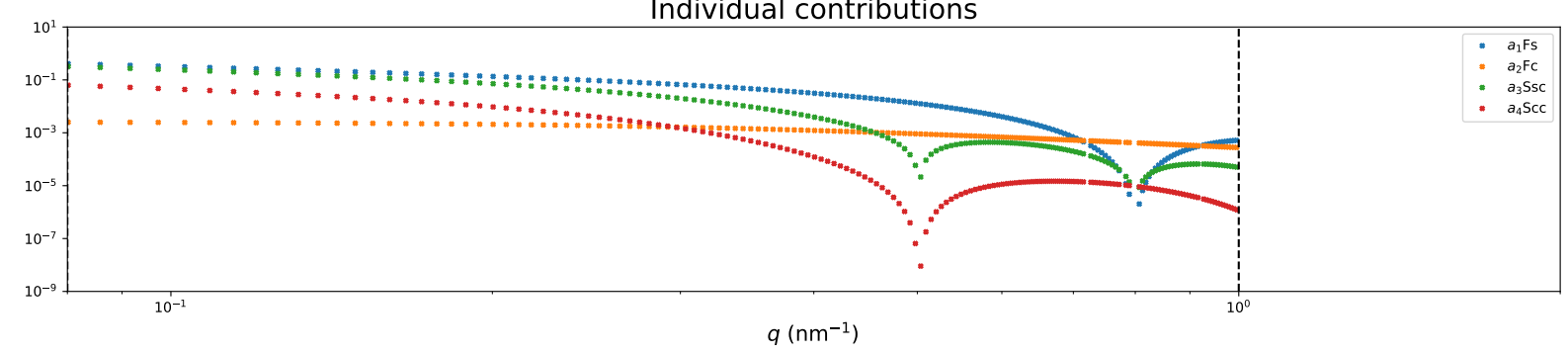

Results from the micelle model fitted to the data. In the upper image, all scattering profiles of the individual experiments plus the fit curve are shown. In the lower graphic, the individual contributions of the four terms $\left(F_{c}, F_{s}, S_{s c}\right.$ and $S_{c c}$ ) are displayed.

Salt: $\mathrm{CaCl}_{2}$ concentration: $1 . \mathrm{mM}$

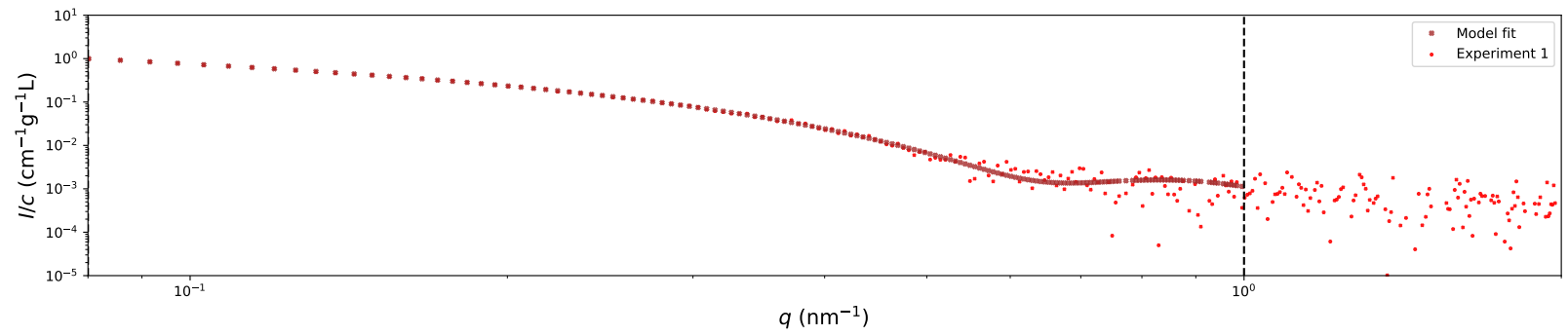

Individual contributions

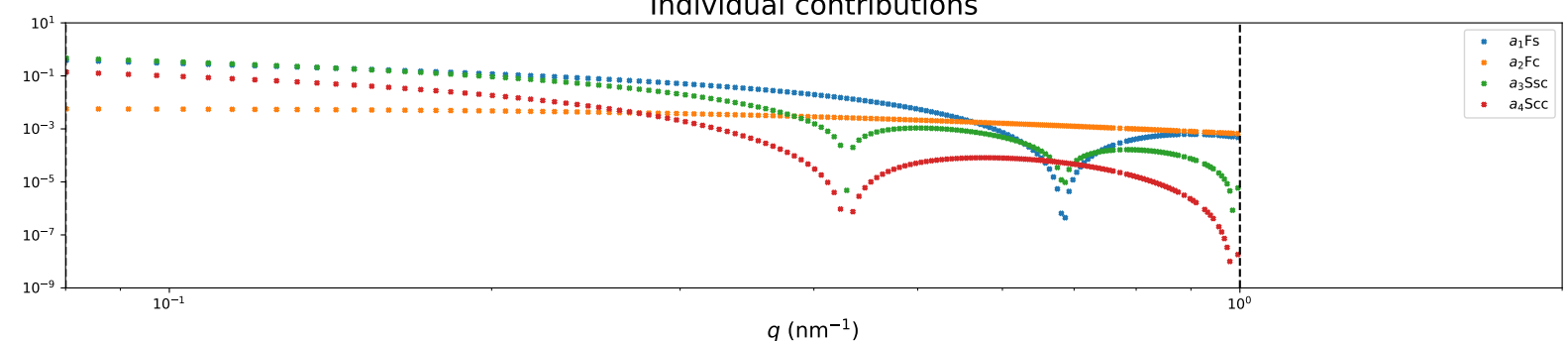

Results from the micelle model fitted to the data. In the upper image, all scattering profiles of the individual experiments plus the fit curve are shown. In the lower graphic, the individual contributions of the four terms $\left(F_{c}, F_{s}, S_{s c}\right.$ and $S_{c c}$ ) are displayed. 
Salt: $\mathrm{CaCl}_{2}$ concentration: $1.5 \mathrm{mM}$

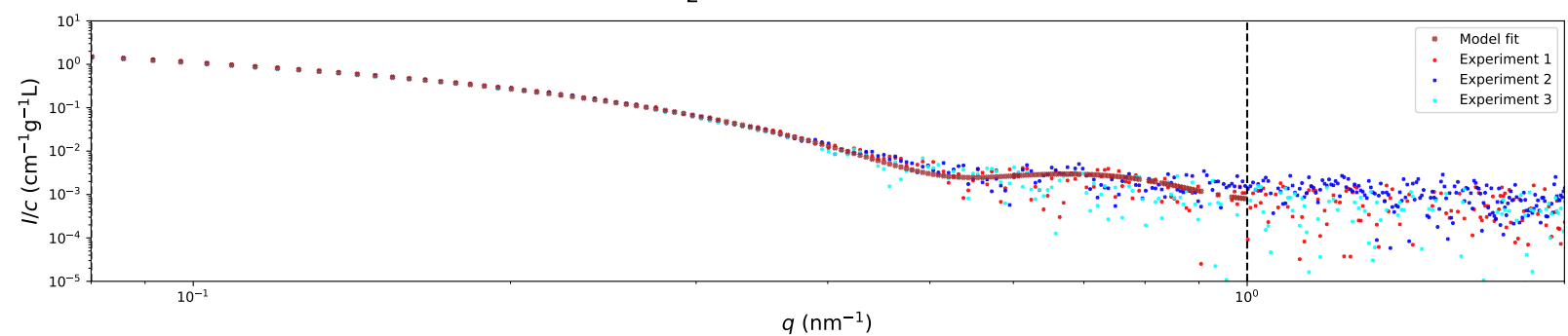

Individual contributions

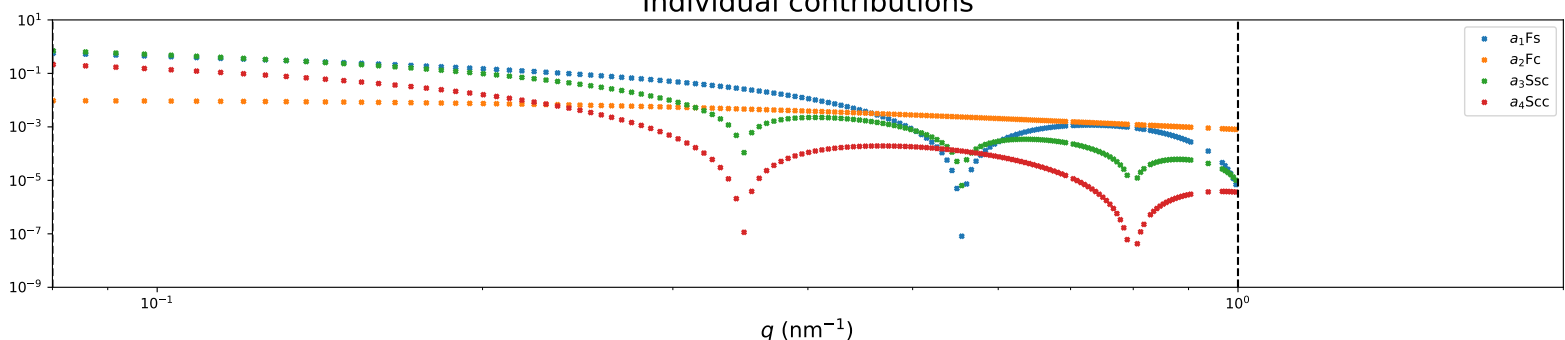

Results from the micelle model fitted to the data. In the upper image, all scattering profiles of the individual experiments plus the fit curve are shown. In the lower graphic, the individual contributions of the four terms $\left(F_{c}, F_{s}, S_{s c}\right.$ and $S_{c c}$ ) are displayed.

Salt: $\mathrm{CaCl}_{2}$ concentration: $2.0 \mathrm{mM}$

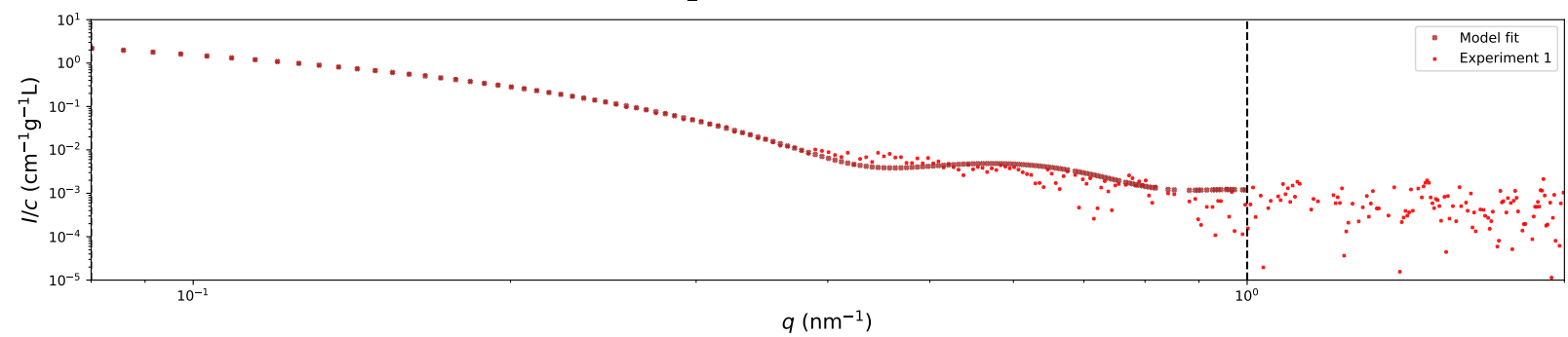

Individual contributions

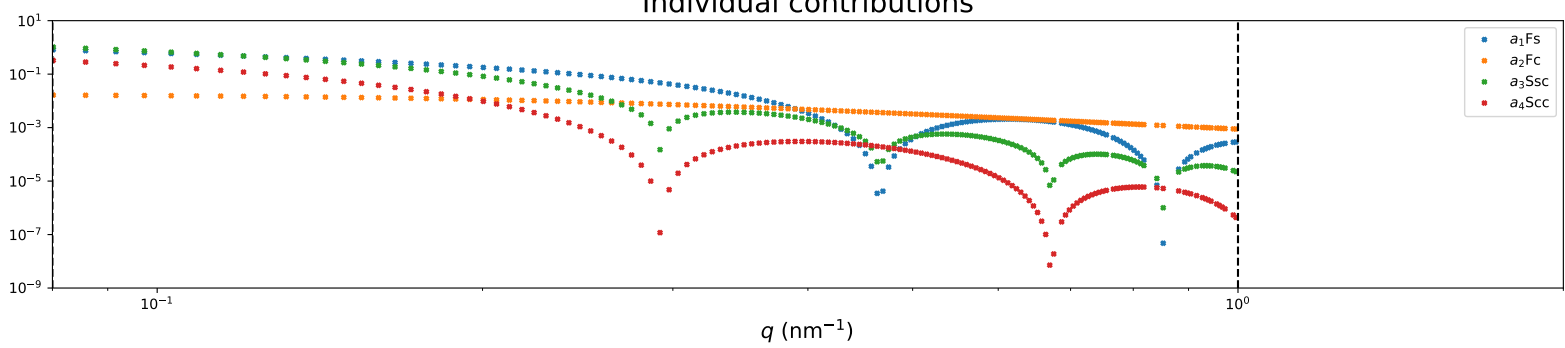

Results from the micelle model fitted to the data. In the upper image, all scattering profiles of the individual experiments plus the fit curve are shown. In the lower graphic, the individual contributions of the four terms $\left(F_{c}, F_{s}, S_{s c}\right.$ and $S_{c c}$ ) are displayed. 
Salt: $\mathrm{CaCl}_{2}$ concentration: $2.5 \mathrm{mM}$

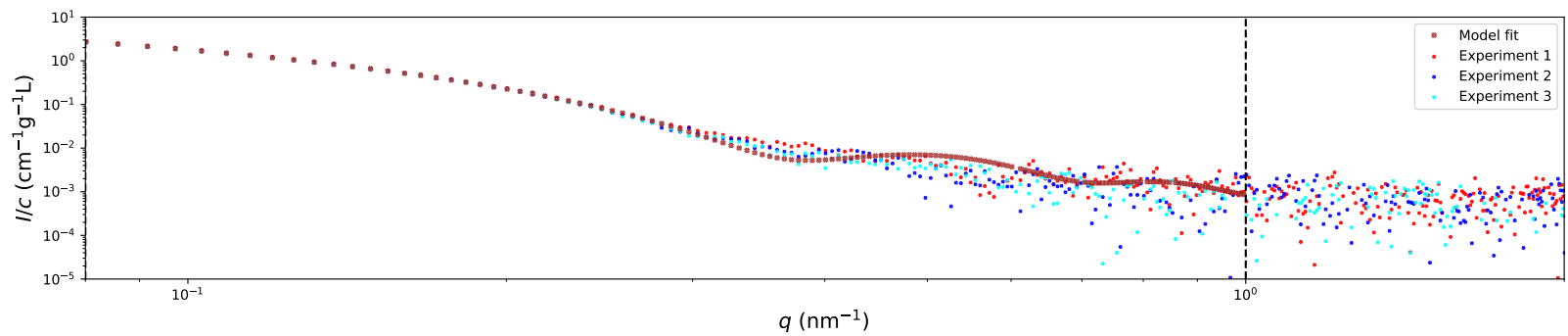

Individual contributions

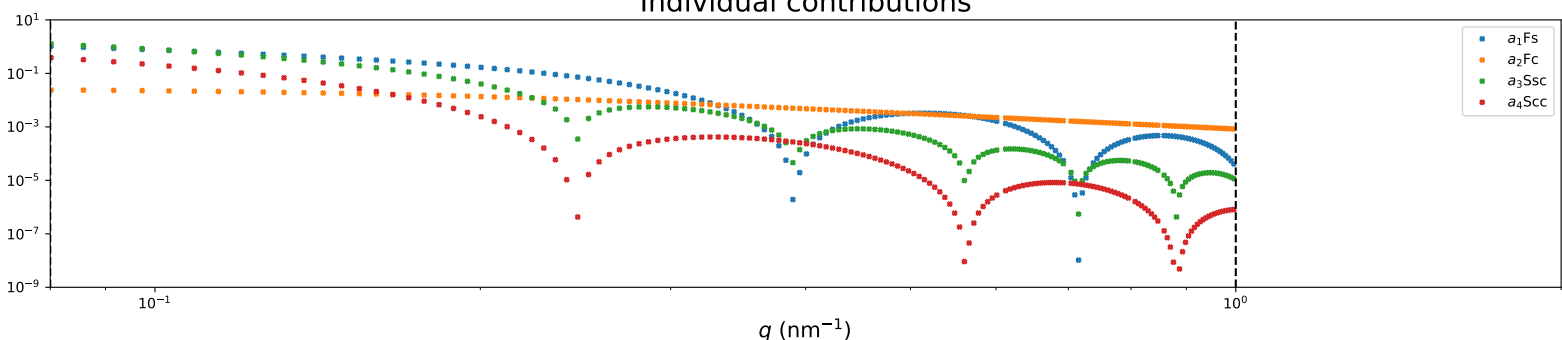

Results from the micelle model fitted to the data. In the upper image, all scattering profiles of the individual experiments plus the fit curve are shown. In the lower graphic, the individual contributions of the four terms $\left(F_{c}, F_{s}, S_{s c}\right.$ and $S_{c c}$ ) are displayed.

Salt: $\mathrm{CaCl}_{2}$ concentration: $4 \mathrm{mM}$

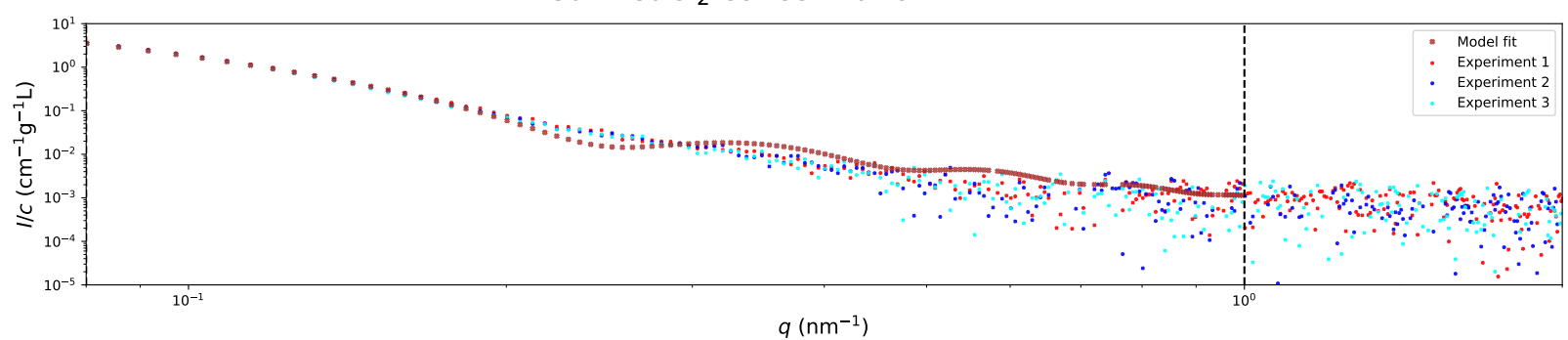

Individual contributions

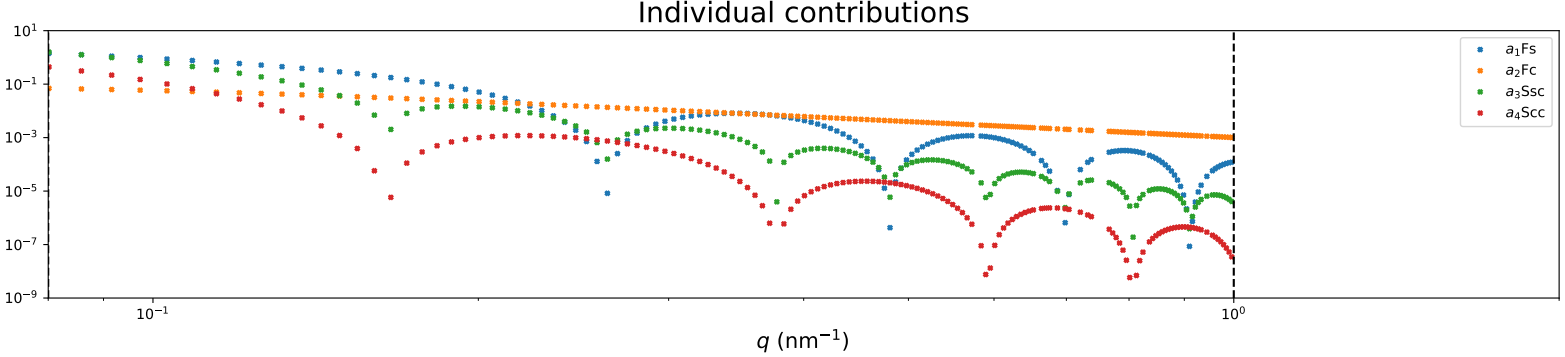

Results from the micelle model fitted to the data. In the upper image, all scattering profiles of the individual experiments plus the fit curve are shown. In the lower graphic, the individual contributions of the four terms $\left(F_{c}, F_{s}, S_{s c}\right.$ and $S_{c c}$ ) are displayed. 
Salt: $\mathrm{CaCl}_{2}$ concentration: $5 \mathrm{mM}$

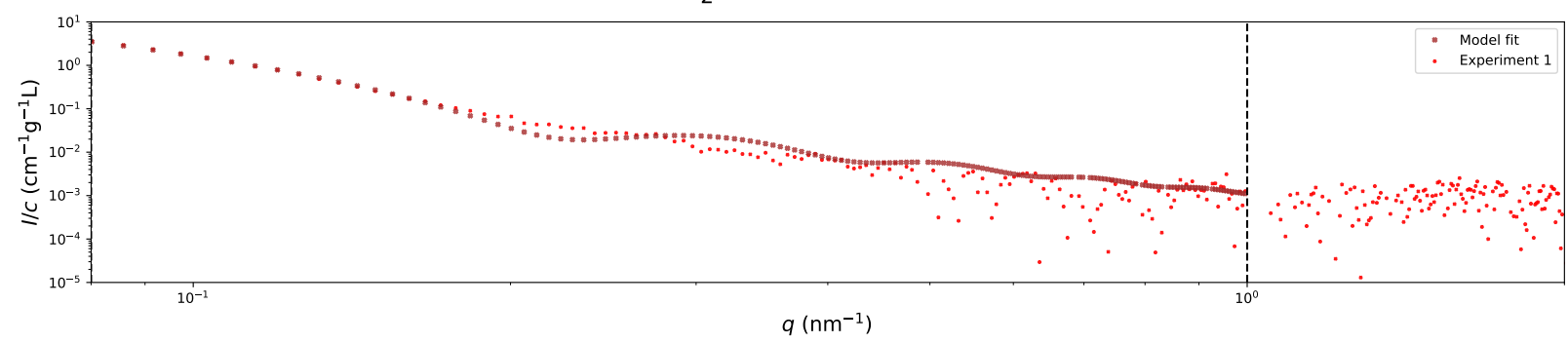

Individual contributions

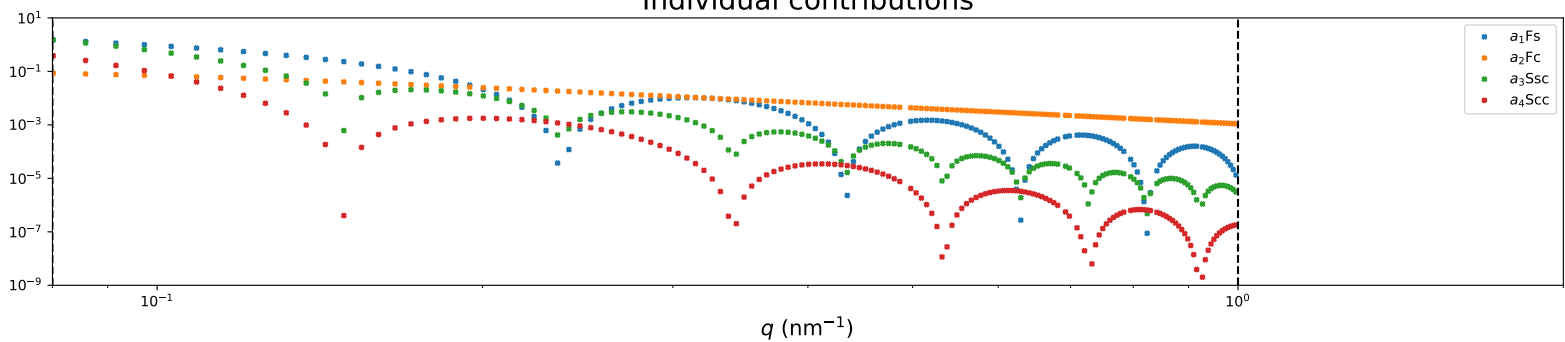

Results from the micelle model fitted to the data. In the upper image, all scattering profiles of the individual experiments plus the fit curve are shown. In the lower graphic, the individual contributions of the four terms $\left(F_{c}, F_{s}, S_{s c}\right.$ and $S_{c c}$ ) are displayed.

Salt: $\mathrm{CaCl}_{2}$ concentration: $10 \mathrm{mM}$

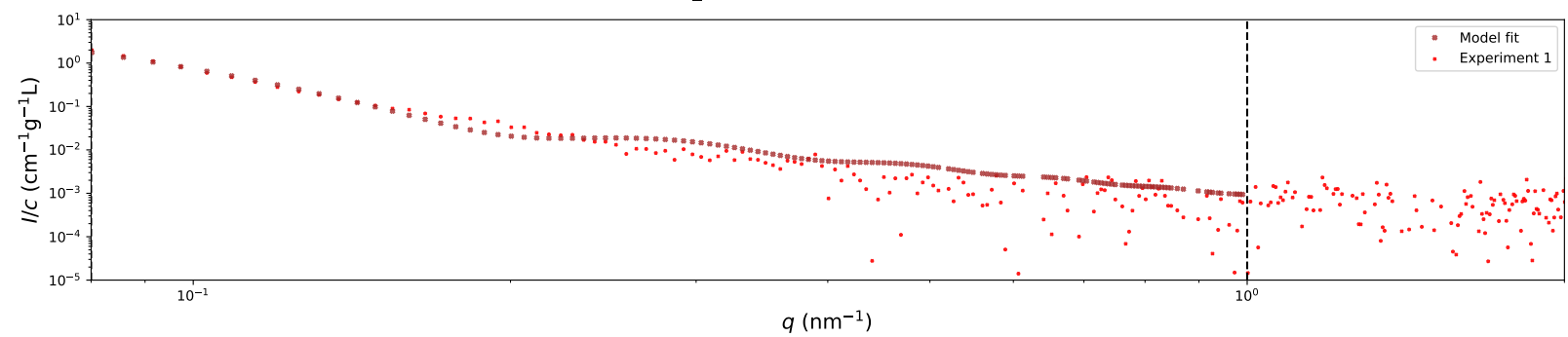

Individual contributions

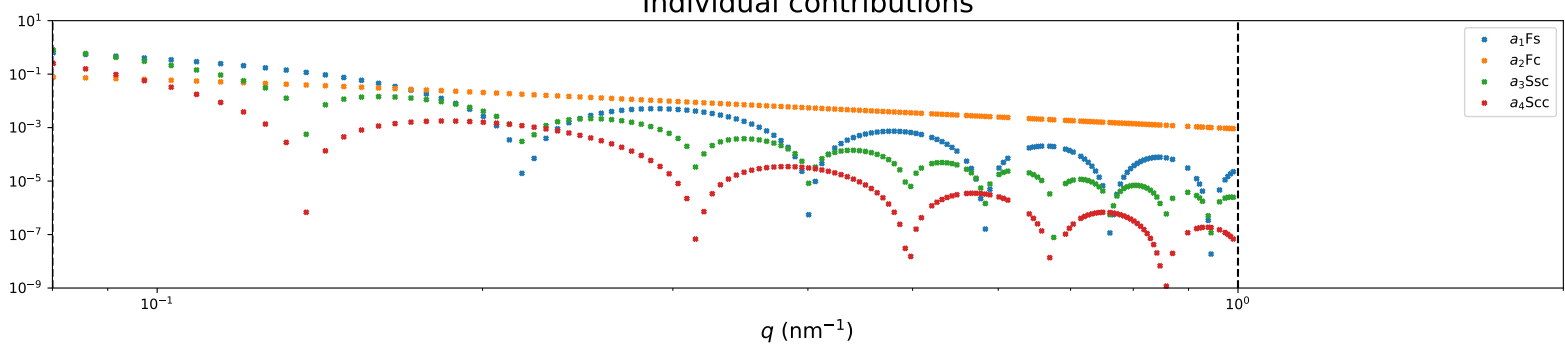

Results from the micelle model fitted to the data. In the upper image, all scattering profiles of the individual experiments plus the fit curve are shown. In the lower graphic, the individual contributions of the four terms $\left(F_{c}, F_{s}, S_{s c}\right.$ and $S_{c c}$ ) are displayed. 


\section{B.5 Model Fits of Vimentin Assembled in the Presence of Hexammine-cobalt(III) chloride}

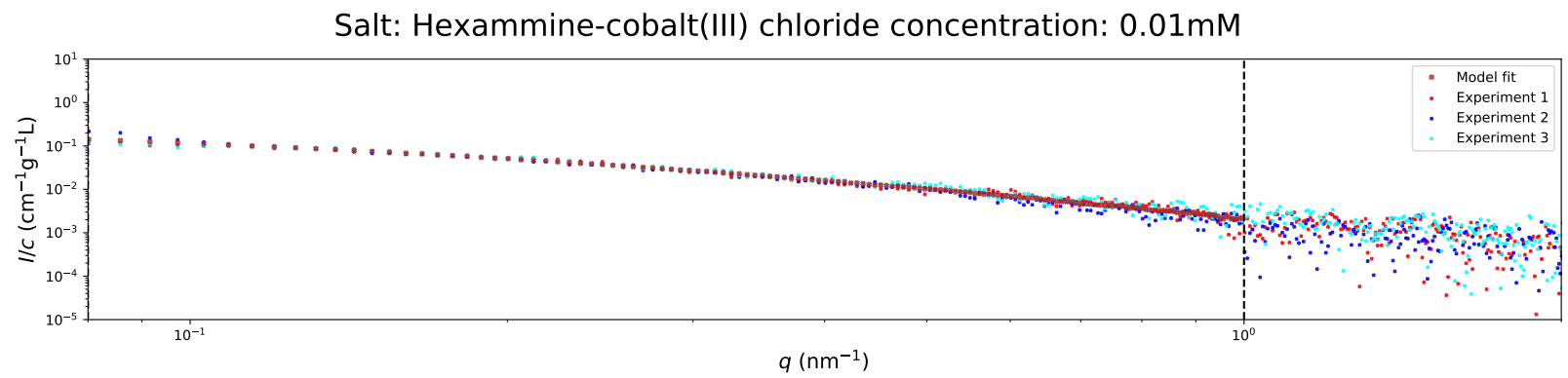

Individual contributions

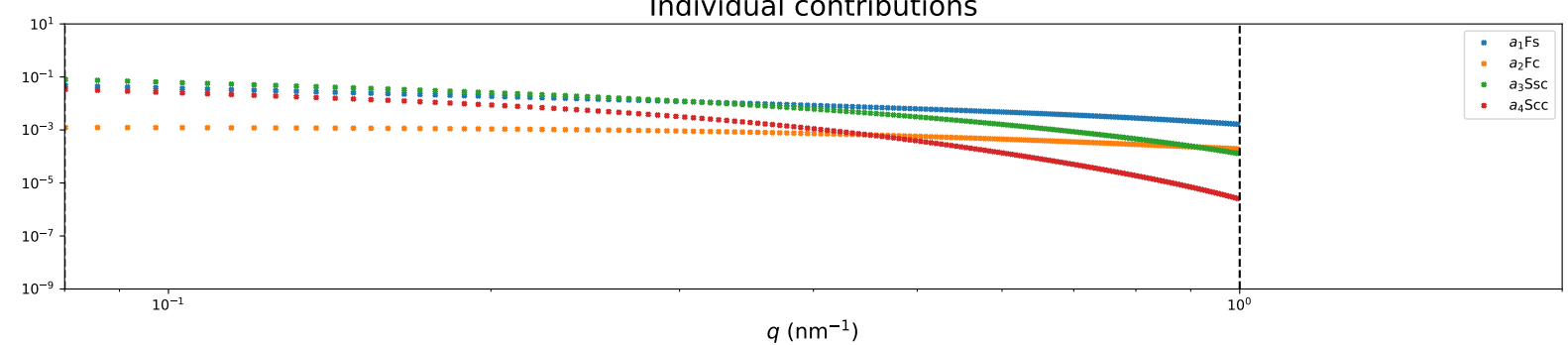

Results from the micelle model fitted to the data. In the upper image, all scattering profiles of the individual experiments plus the fit curve are shown. In the lower graphic, the individual contributions of the four terms $\left(F_{c}, F_{s}, S_{s c}\right.$ and $S_{c c}$ ) are displayed. 
Salt: Hexammine-cobalt(III) chloride concentration: $0.02 \mathrm{mM}$

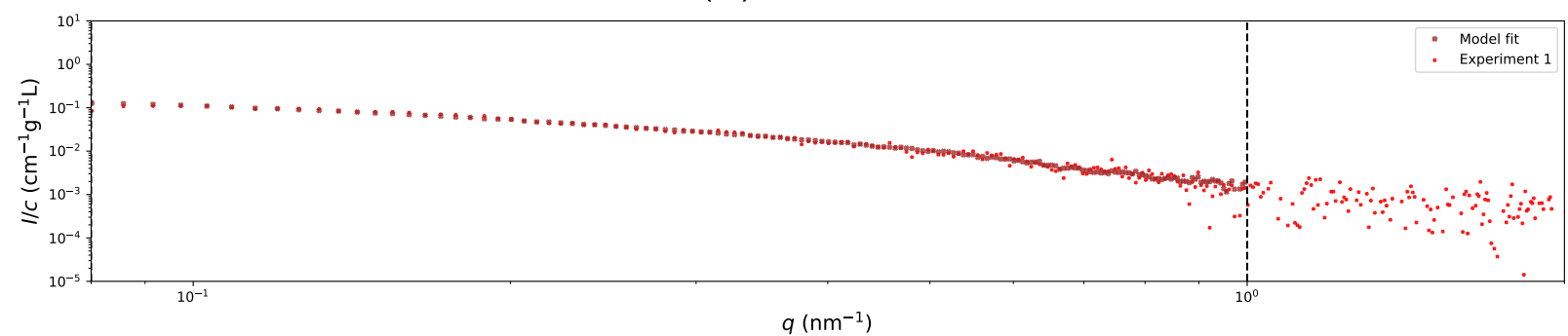

Individual contributions

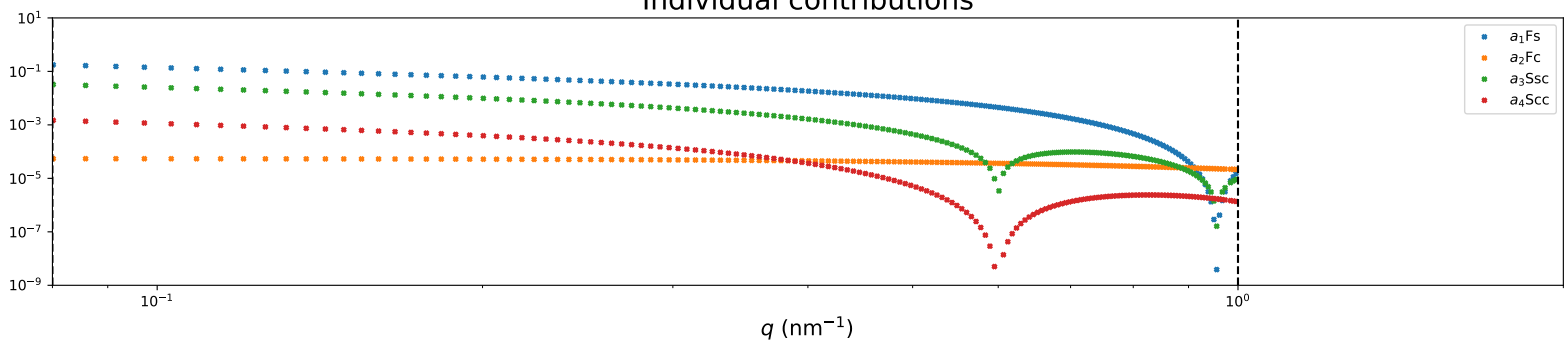

Results from the micelle model fitted to the data. In the upper image, all scattering profiles of the individual experiments plus the fit curve are shown. In the lower graphic, the individual contributions of the four terms $\left(F_{c}, F_{s}, S_{s c}\right.$ and $S_{c c}$ ) are displayed.

Salt: Hexammine-cobalt(III) chloride concentration: $0.03 \mathrm{mM}$

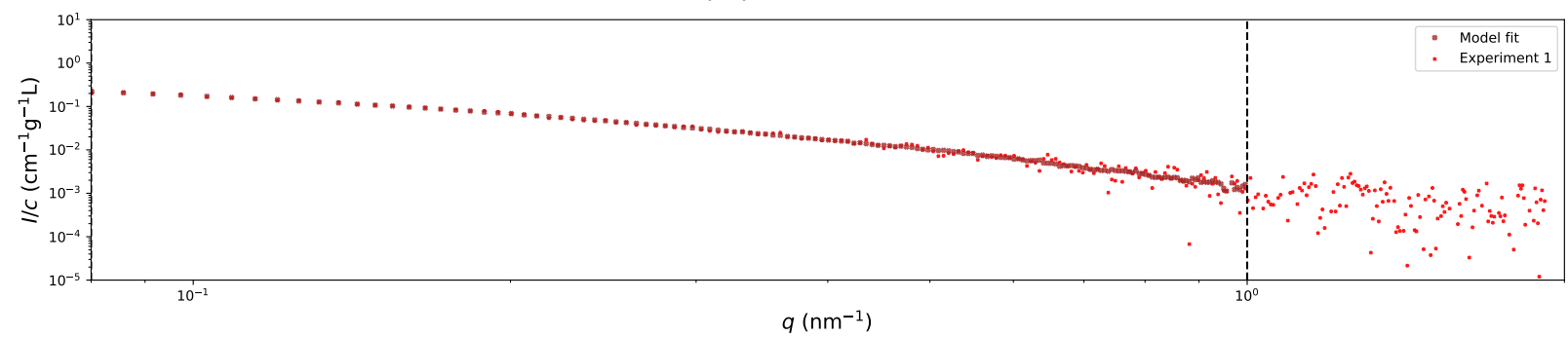

Individual contributions

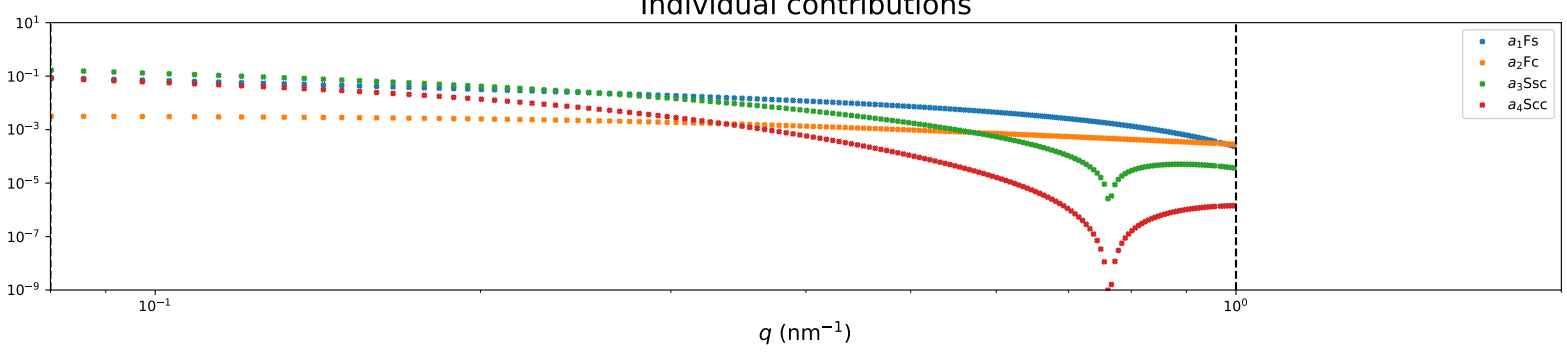

Results from the micelle model fitted to the data. In the upper image, all scattering profiles of the individual experiments plus the fit curve are shown. In the lower graphic, the individual contributions of the four terms $\left(F_{c}, F_{s}, S_{s c}\right.$ and $S_{c c}$ ) are displayed. 
Salt: Hexammine-cobalt(III) chloride concentration: $0.05 \mathrm{mM}$

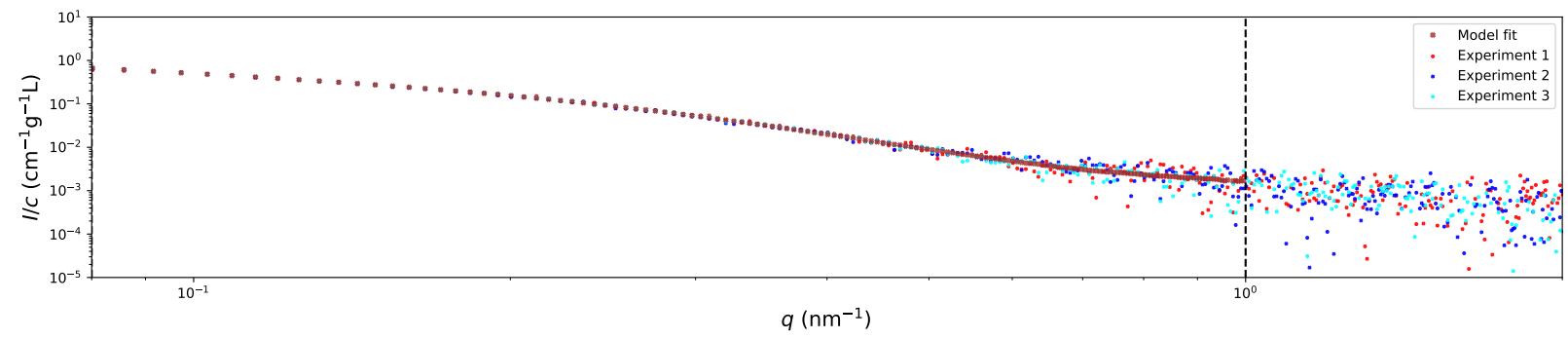

Individual contributions

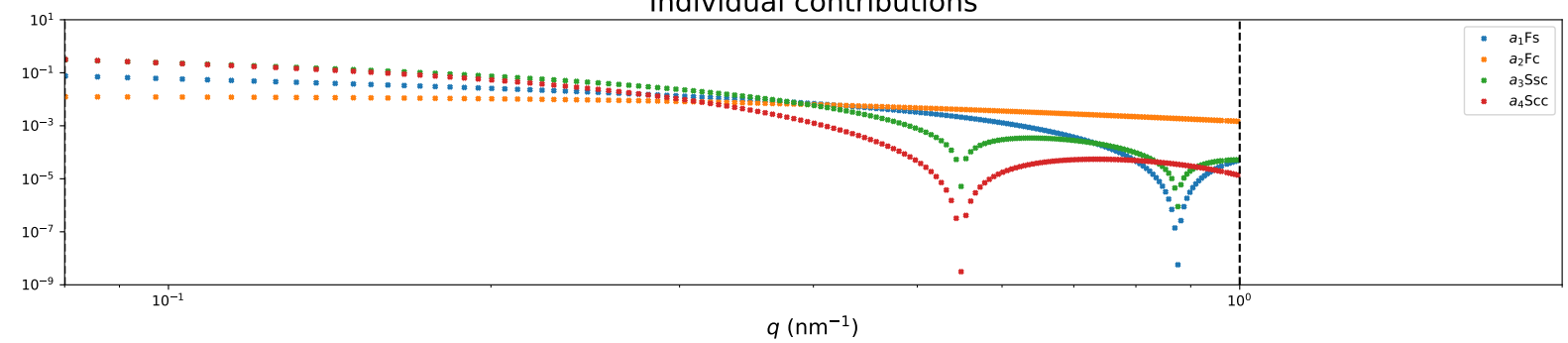

Results from the micelle model fitted to the data. In the upper image, all scattering profiles of the individual experiments plus the fit curve are shown. In the lower graphic, the individual contributions of the four terms $\left(F_{c}, F_{s}, S_{s c}\right.$ and $S_{c c}$ ) are displayed.

Salt: Hexammine-cobalt(III) chloride concentration: $0.08 \mathrm{mM}$

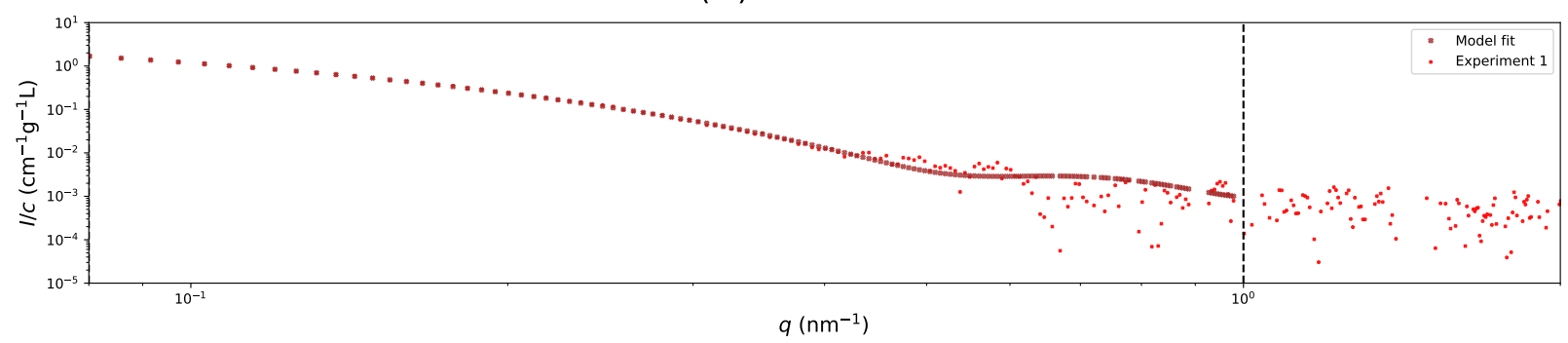

Individual contributions

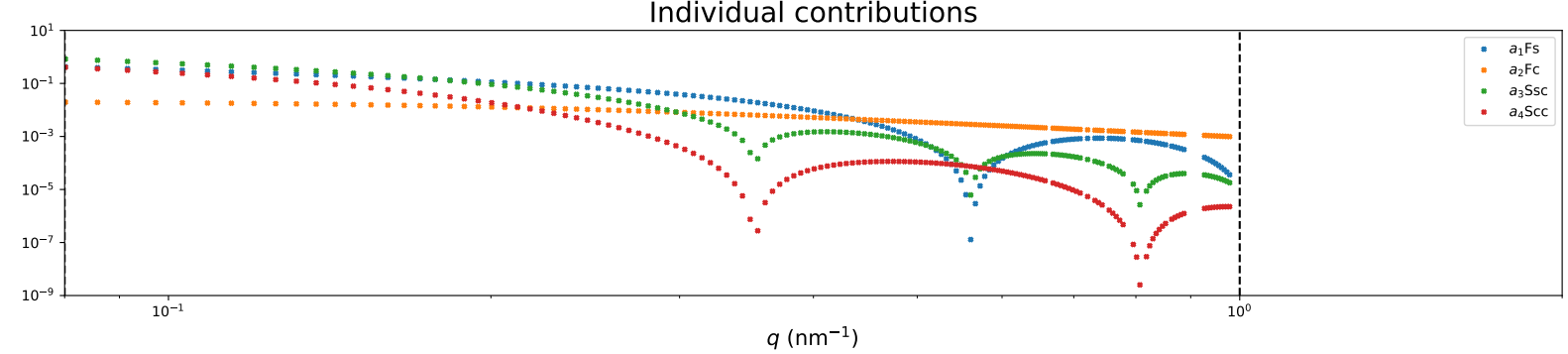

Results from the micelle model fitted to the data. In the upper image, all scattering profiles of the individual experiments plus the fit curve are shown. In the lower graphic, the individual contributions of the four terms $\left(F_{c}, F_{s}, S_{s c}\right.$ and $S_{c c}$ ) are displayed. 
Salt: Hexammine-cobalt(III) chloride concentration: $0.1 \mathrm{mM}$

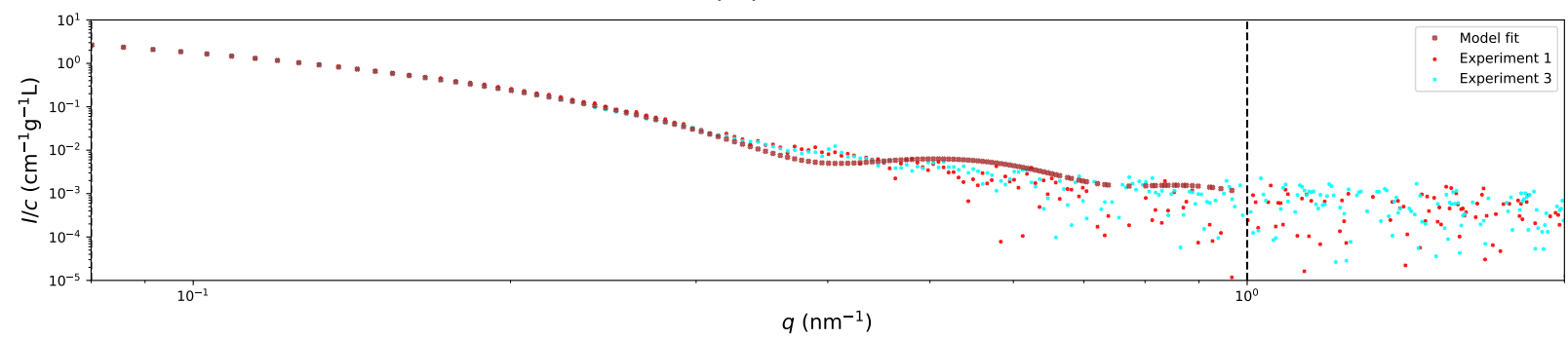

Individual contributions

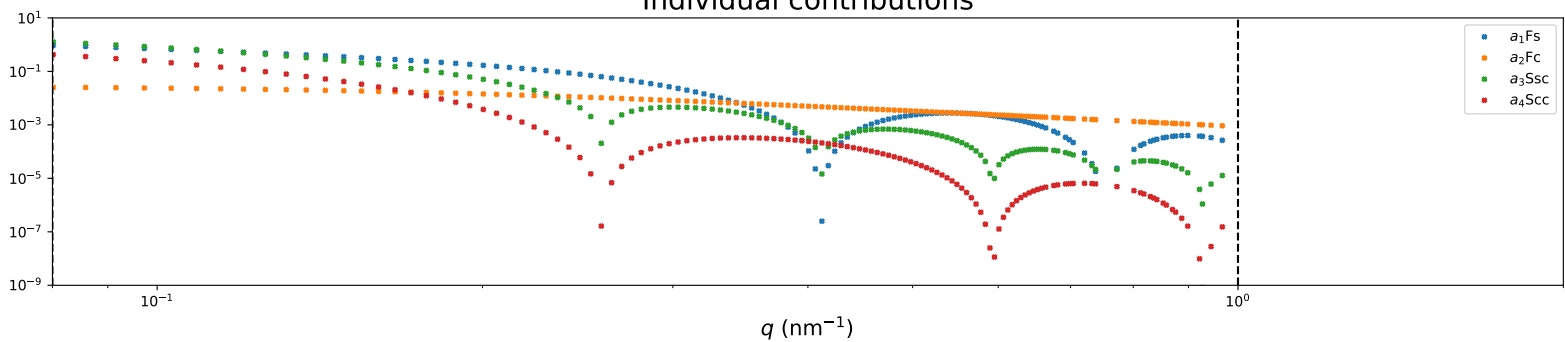

Results from the micelle model fitted to the data. In the upper image, all scattering profiles of the individual experiments plus the fit curve are shown. In the lower graphic, the individual contributions of the four terms $\left(F_{c}, F_{s}, S_{s c}\right.$ and $S_{c c}$ ) are displayed.

Salt: Hexammine-cobalt(III) chloride concentration: $0.2 \mathrm{mM}$

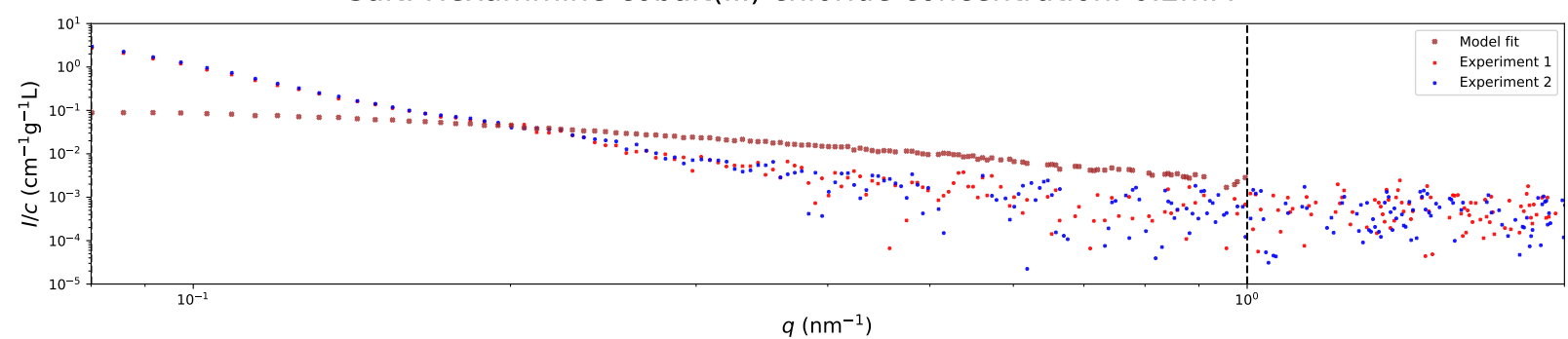

Individual contributions

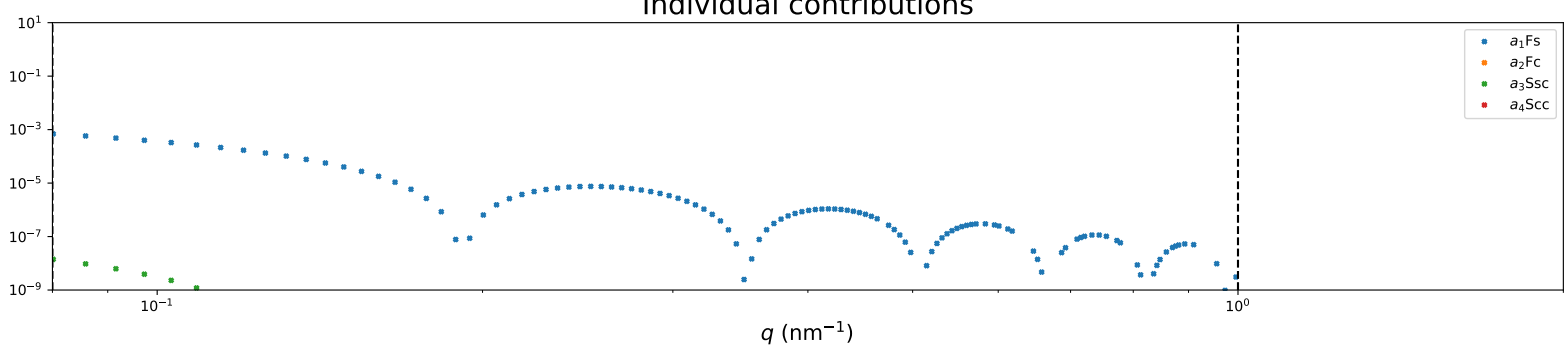

Results from the micelle model fitted to the data. In the upper image, all scattering profiles of the individual experiments plus the fit curve are shown. In the lower graphic, the individual contributions of the four terms $\left(F_{c}, F_{s}, S_{s c}\right.$ and $S_{c c}$ ) are displayed. 
Salt: Hexammine-cobalt(III) chloride concentration: $0.5 \mathrm{mM}$
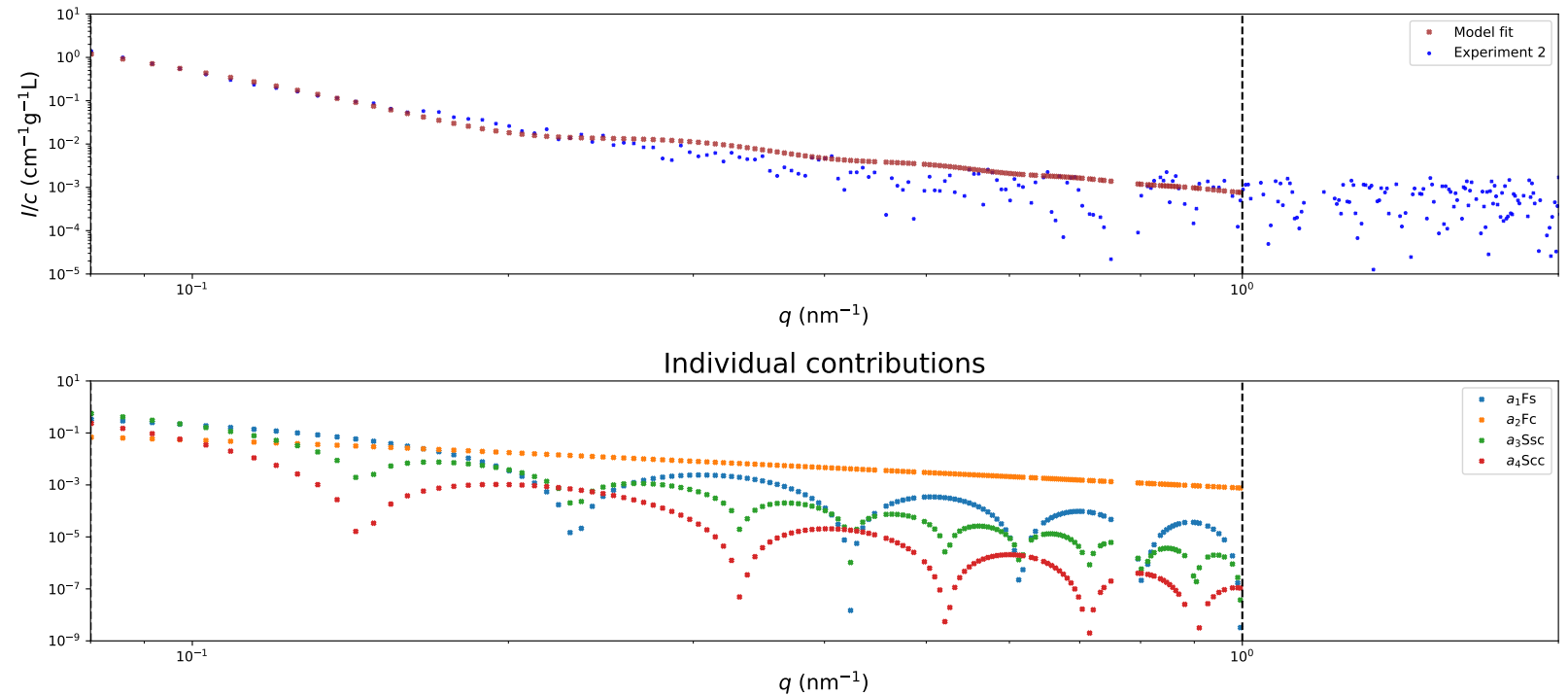

Results from the micelle model fitted to the data. In the upper image, all scattering profiles of the individual experiments plus the fit curve are shown. In the lower graphic, the individual contributions of the four terms $\left(F_{c}, F_{s}, S_{s c}\right.$ and $S_{c c}$ ) are displayed. 


\section{B.6 Model Fits of Vimentin Assembled in the Presence of Sper- mine}

Salt: Spermine concentration: $0.01 \mathrm{mM}$

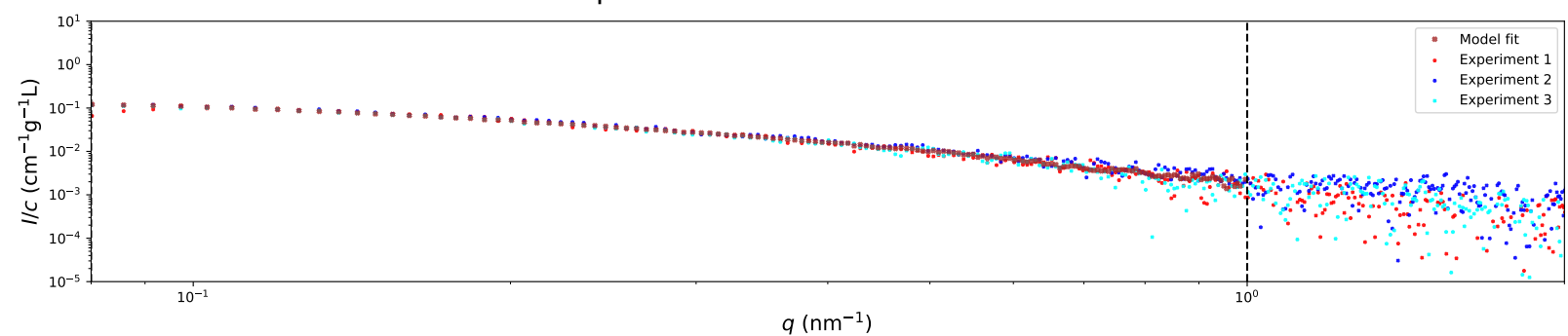

Individual contributions

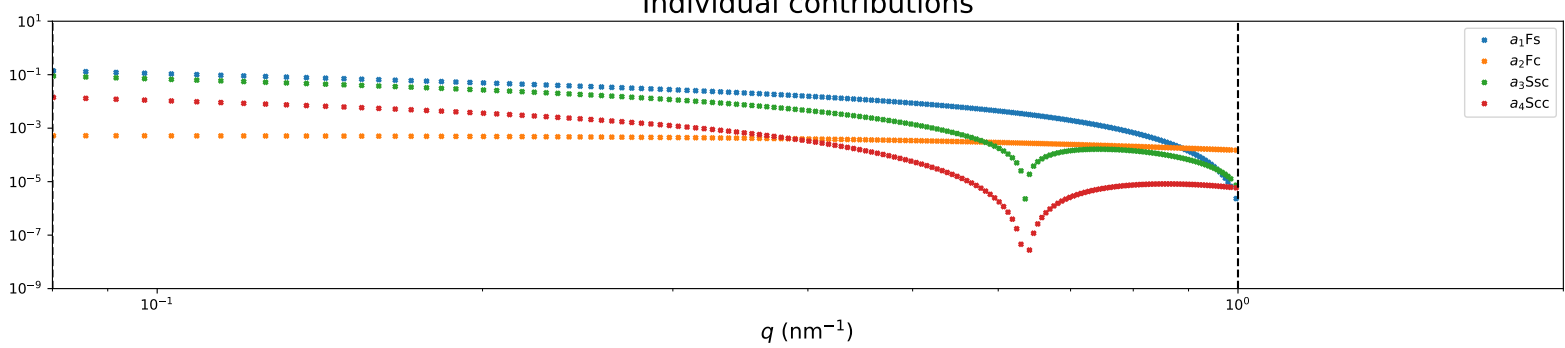

Results from the micelle model fitted to the data. In the upper image, all scattering profiles of the individual experiments plus the fit curve are shown. In the lower graphic, the individual contributions of the four terms $\left(F_{c}, F_{s}, S_{s c}\right.$ and $S_{c c}$ ) are displayed.

Salt: Spermine concentration: $0.02 \mathrm{mM}$

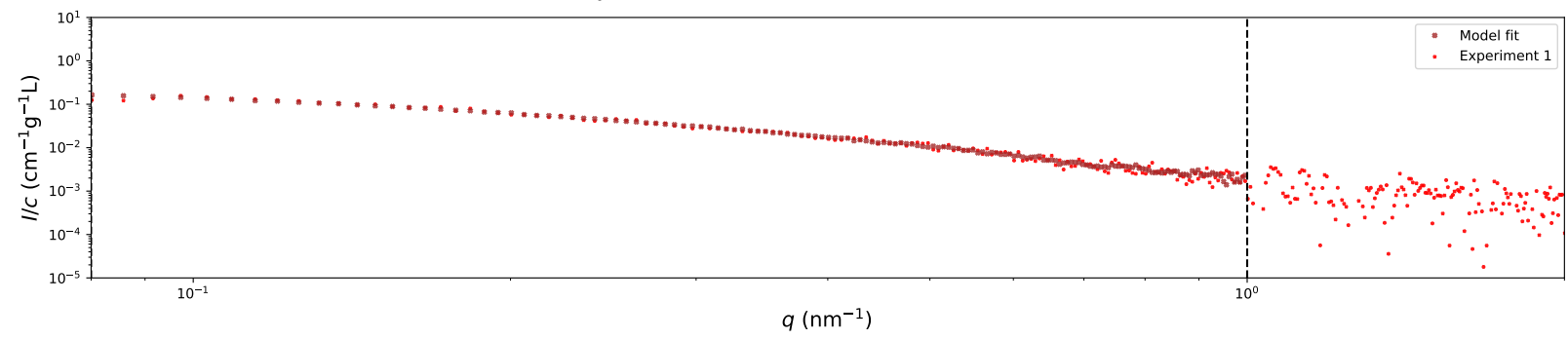

Individual contributions

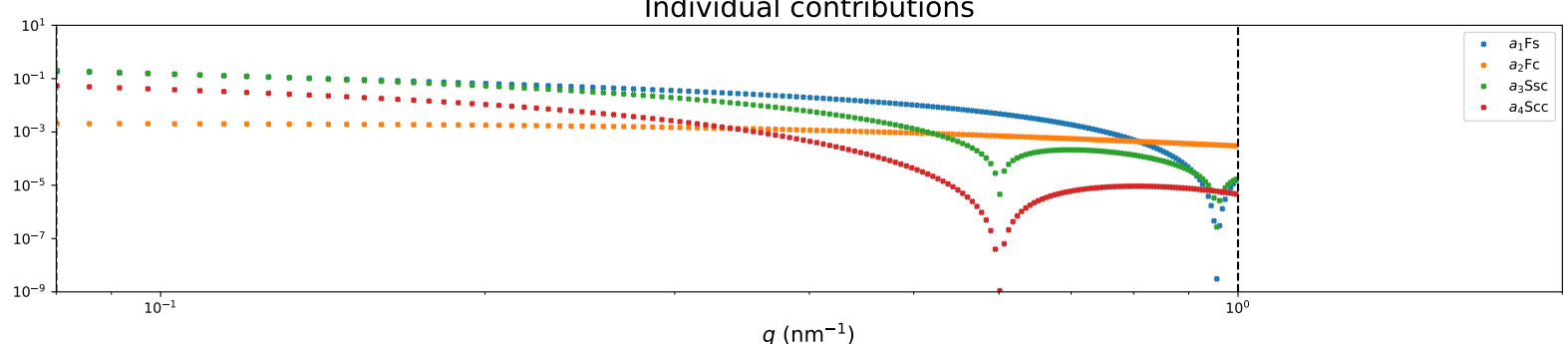

Results from the micelle model fitted to the data. In the upper image, all scattering profiles of the individual experiments plus the fit curve are shown. In the lower graphic, the individual contributions of the four terms $\left(F_{c}, F_{s}, S_{s c}\right.$ and $S_{c c}$ ) are displayed. 
Salt: Spermine concentration: $0.03 \mathrm{mM}$

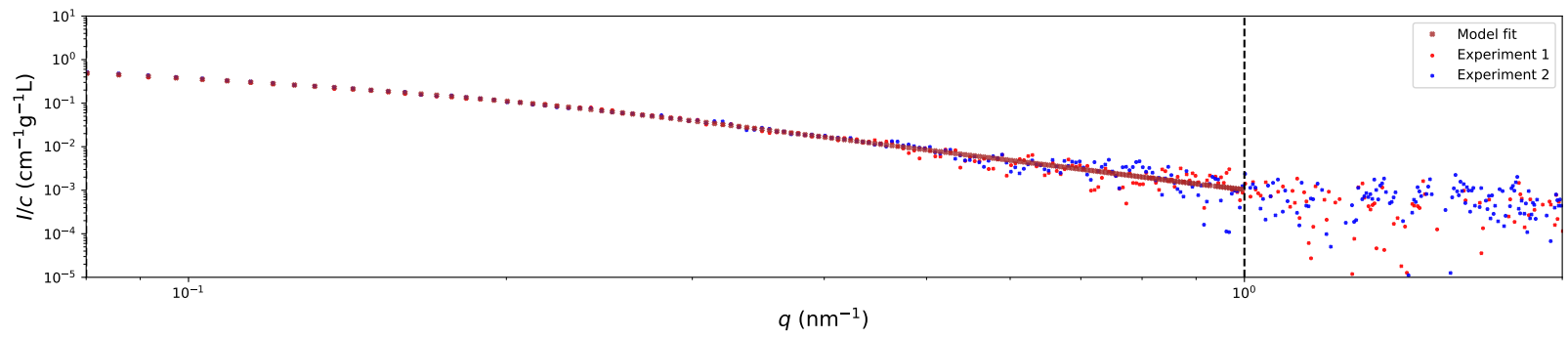

Individual contributions

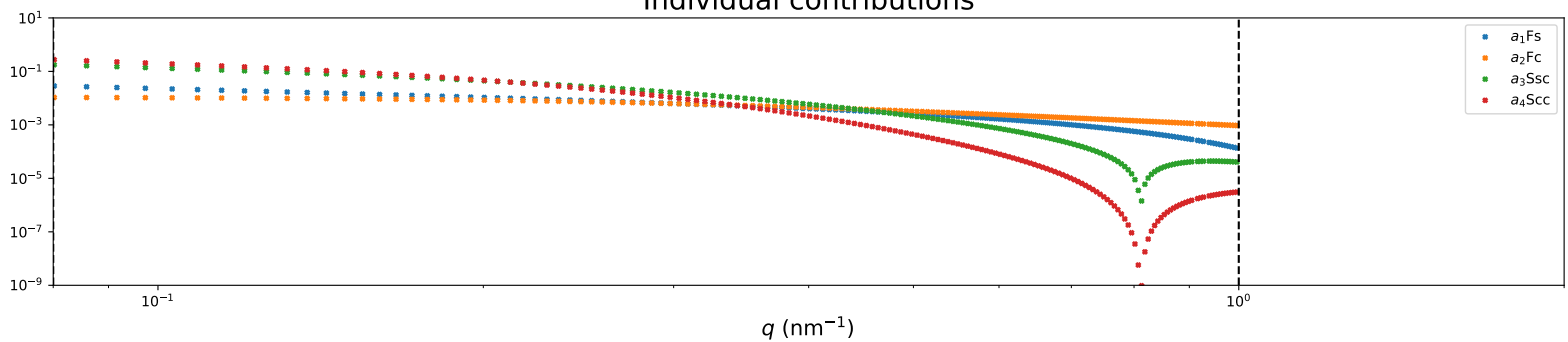

Results from the micelle model fitted to the data. In the upper image, all scattering profiles of the individual experiments plus the fit curve are shown. In the lower graphic, the individual contributions of the four terms $\left(F_{c}, F_{s}, S_{s c}\right.$ and $S_{c c}$ ) are displayed.

Salt: Spermine concentration: $0.04 \mathrm{mM}$

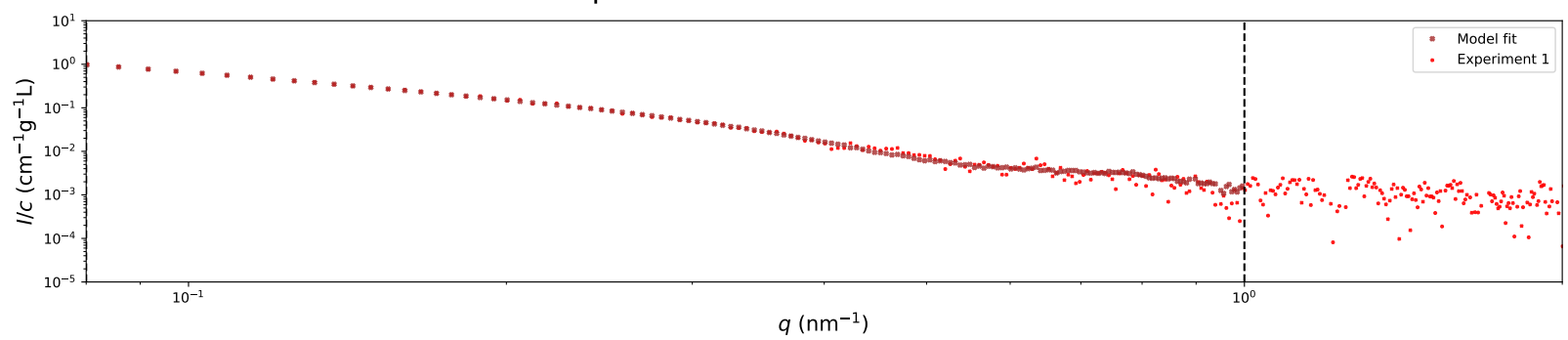

Individual contributions

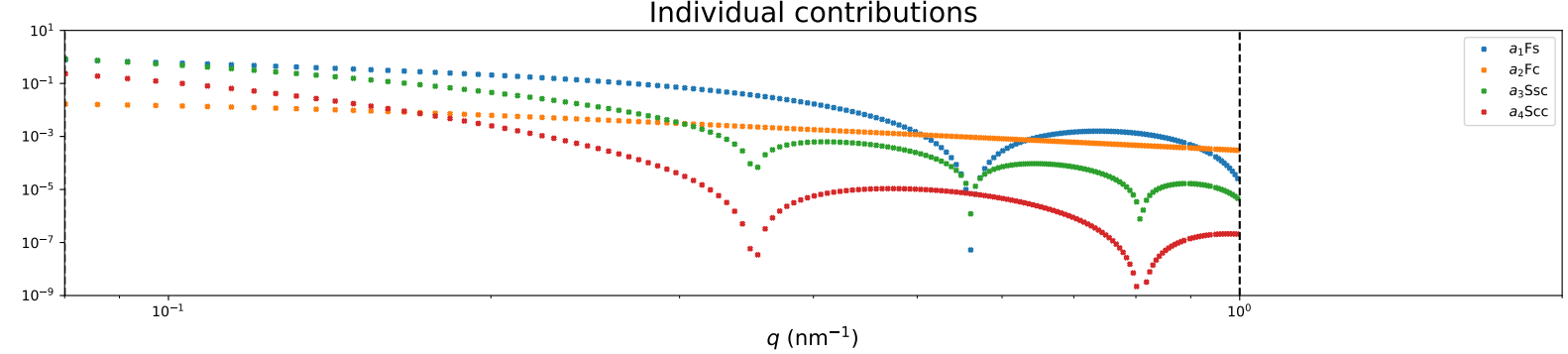

Results from the micelle model fitted to the data. In the upper image, all scattering profiles of the individual experiments plus the fit curve are shown. In the lower graphic, the individual contributions of the four terms $\left(F_{c}, F_{s}, S_{s c}\right.$ and $S_{c c}$ ) are displayed. 
Salt: Spermine concentration: $0.05 \mathrm{mM}$

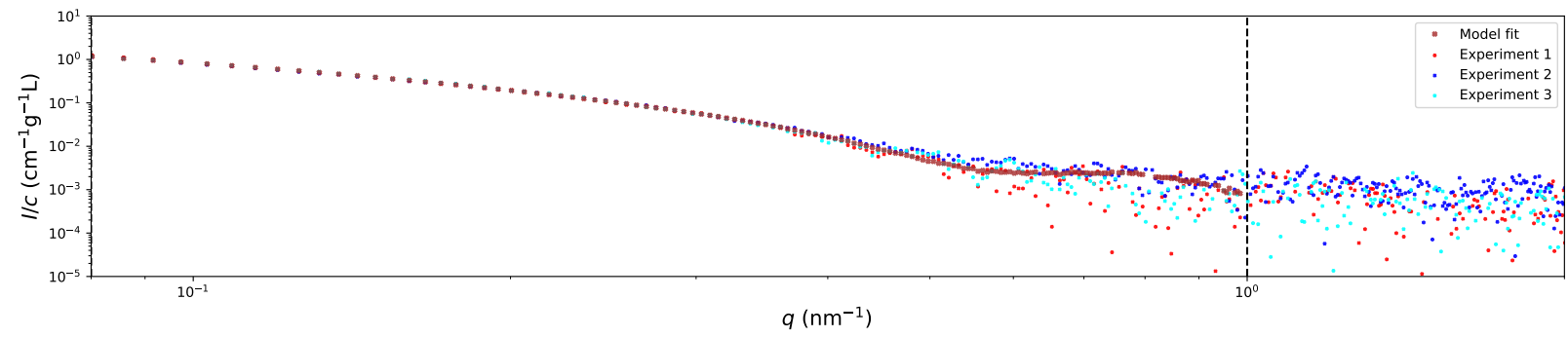

Individual contributions

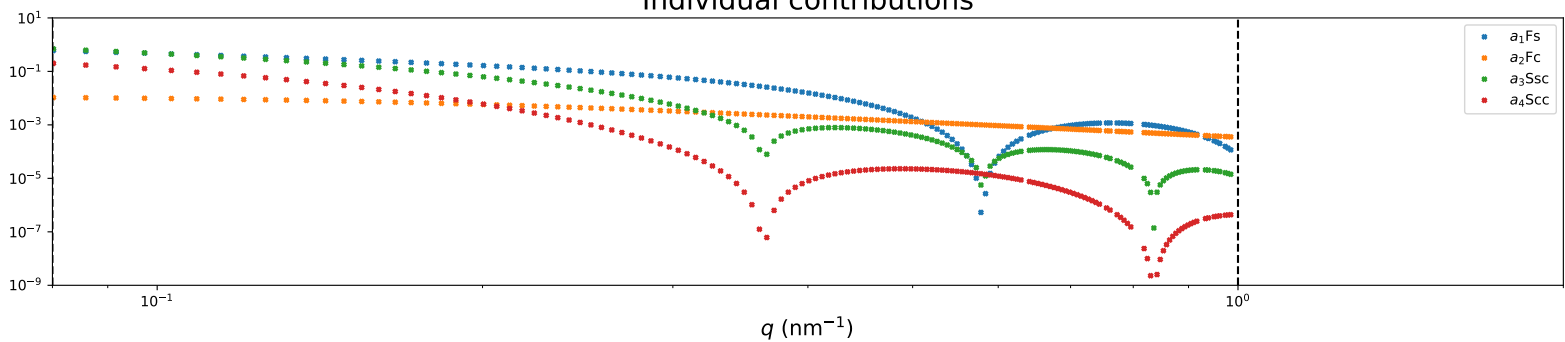

Results from the micelle model fitted to the data. In the upper image, all scattering profiles of the individual experiments plus the fit curve are shown. In the lower graphic, the individual contributions of the four terms $\left(F_{c}, F_{s}, S_{s c}\right.$ and $S_{c c}$ ) are displayed.

Salt: Spermine concentration: $0.08 \mathrm{mM}$

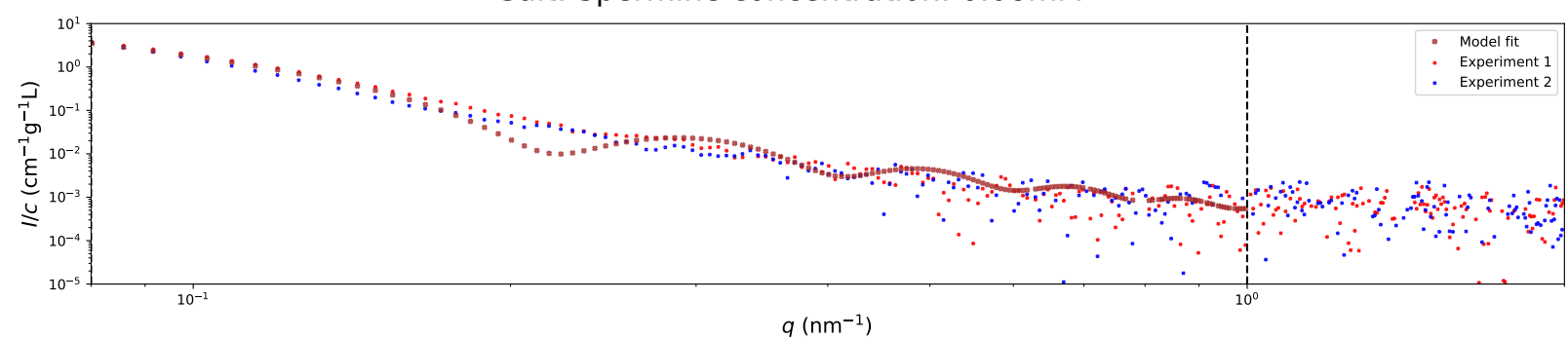

Individual contributions

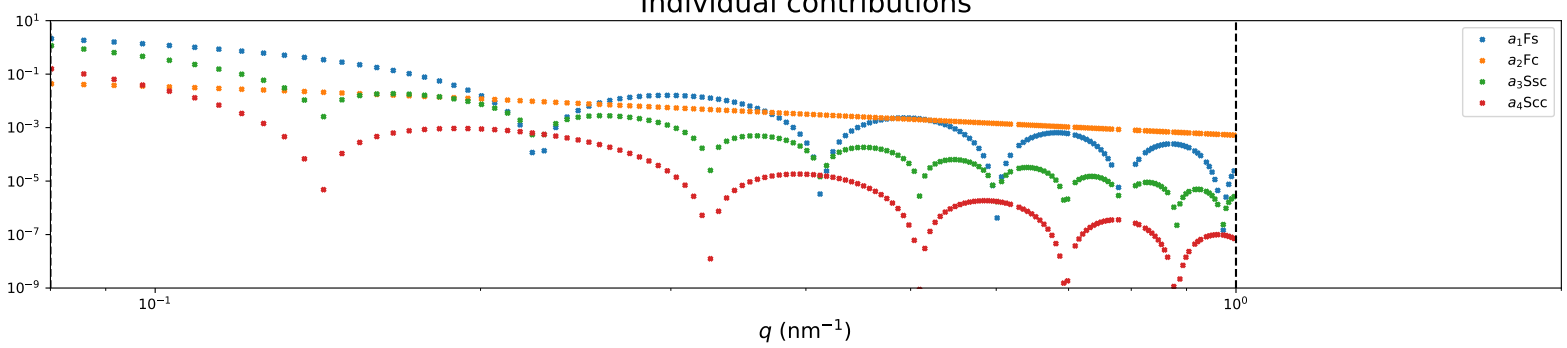

Results from the micelle model fitted to the data. In the upper image, all scattering profiles of the individual experiments plus the fit curve are shown. In the lower graphic, the individual contributions of the four terms $\left(F_{c}, F_{s}, S_{s c}\right.$ and $S_{c c}$ ) are displayed. 
Salt: Spermine concentration: $0.09 \mathrm{mM}$

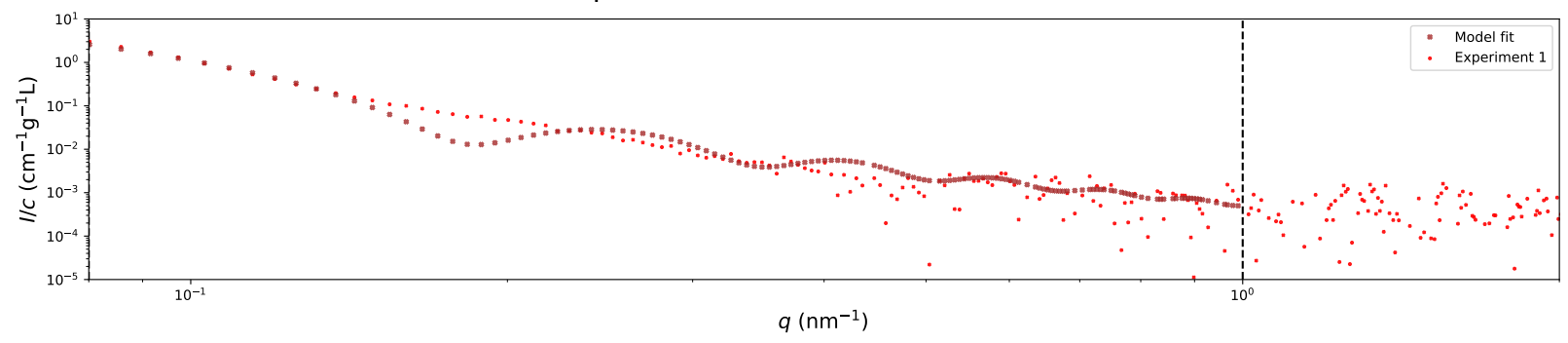

Individual contributions

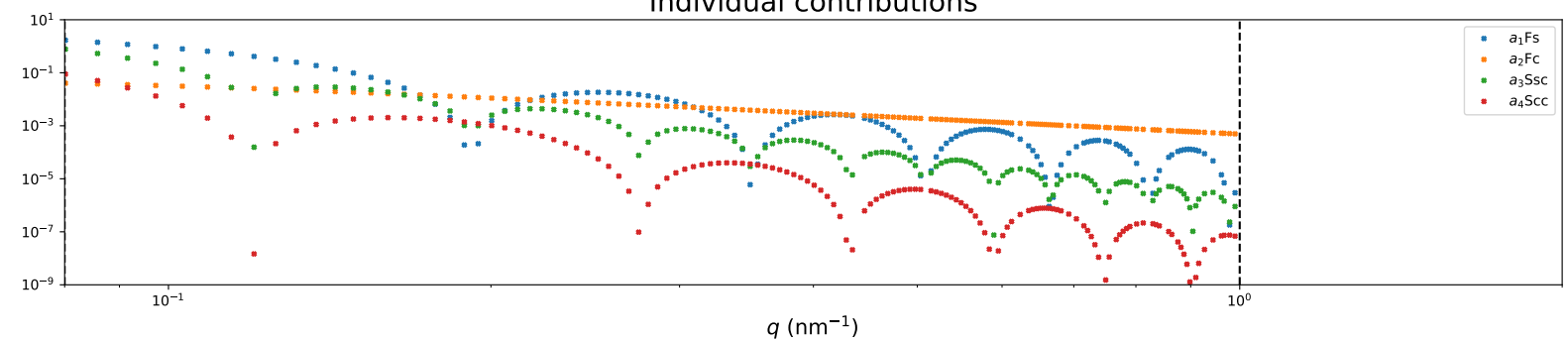

Results from the micelle model fitted to the data. In the upper image, all scattering profiles of the individual experiments plus the fit curve are shown. In the lower graphic, the individual contributions of the four terms $\left(F_{c}, F_{s}, S_{s c}\right.$ and $S_{c c}$ ) are displayed.

Salt: Spermine concentration: $0.1 \mathrm{mM}$

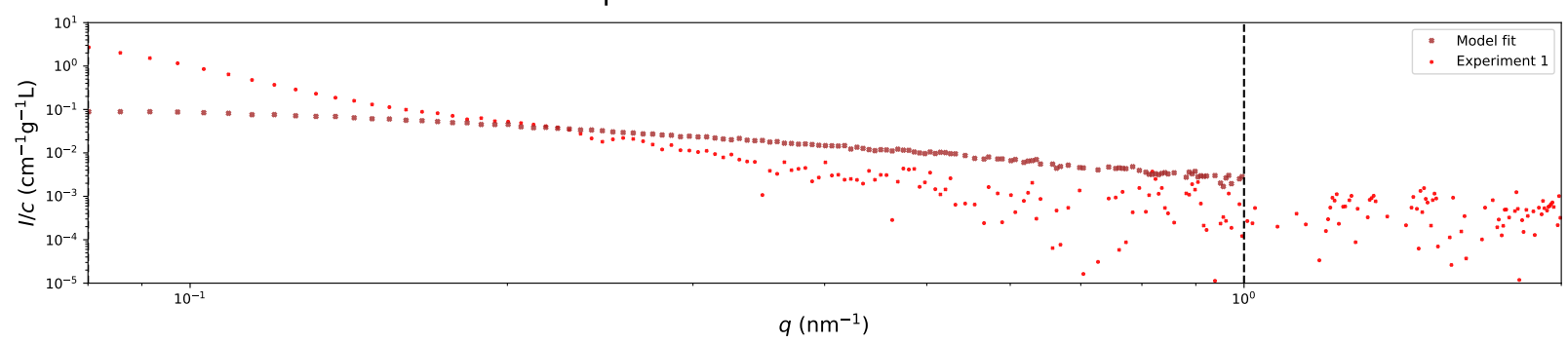

Individual contributions

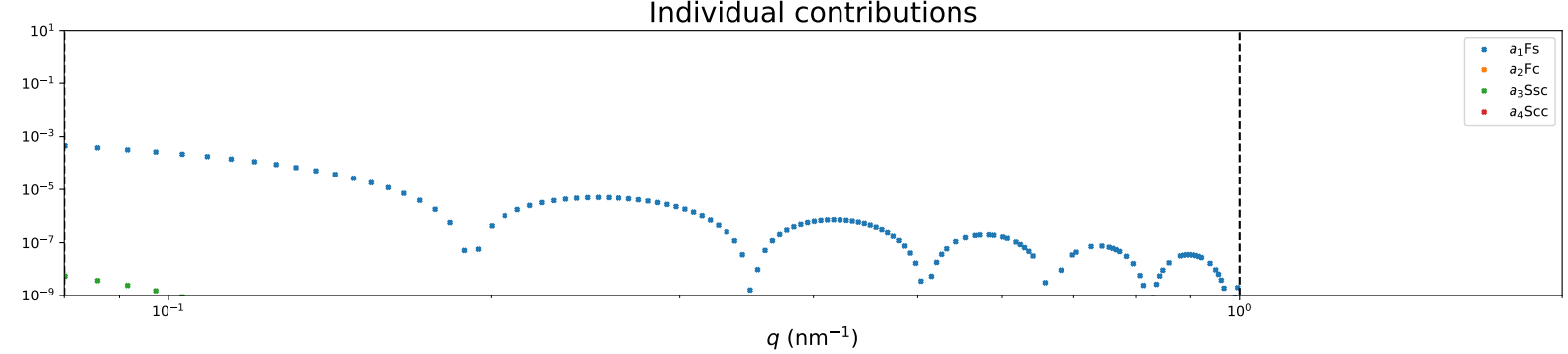

Results from the micelle model fitted to the data. In the upper image, all scattering profiles of the individual experiments plus the fit curve are shown. In the lower graphic, the individual contributions of the four terms $\left(F_{c}, F_{s}, S_{s c}\right.$ and $S_{c c}$ ) are displayed. 


\section{List of Publications}

- Manuela Denz, Gerrit Brehm, Clément Y. J. Hémonnot, Heidi Spears, Andrew Wittmeier, Chiara Cassini, Oliva Saldanha, Eleonora Perego, Ana Diaz, Manfred Burghammer and Sarah Köster. Cyclic olefin copolymer as an X-ray compatible material for microfluidic devices. Lab Chip, 18, 171-178 (2018). DOI: 10.1039/C7LC00824D 


\section{Acknowledgement}

Without the help of many, I would not have been able to make this work as it is. I would like to thank all the contributors, collaborators and friends.

First of all, I would like to thank Prof. Sarah Köster, who gave me the opportunity to work on this project in her group and present it at several conferences. Thanks for the fruitful discussions and all the support during my PhD time. I thank Prof. Thomas Burg for being part of my Thesis Advisory Committee and for being the second reviewer of my thesis. For all the helpful suggestions and comments regarding my project I am very thankful. Furthermore, I would like to thank Prof. Ralf Ficner for being the third member of my Thesis Advisory Committee and a member of my examination board. Thank you for all the interesting discussions during the meetings. I would like to thank Prof. Tim Salditt, Prof. Andreas Janshoff and Prof. Helmut Grubmüller for being part of my examination board

Many thanks to Prof. Harald Herrmann and Dr. Norbert Mücke for all the useful discussions during the IF meetings and also for providing the vimentin plasmids. A huge thanks to the super support, the long discussions and help at the beamlines from Dr. Ana Diaz (cSAXS, PSI) and Dr. Manfred Burghammer (ID13, ESRF). For the kind introduction to the AFM, I would like to thank the whole group of Andreas Janshoff.

A big thanks to Manuel Marschall. Not only for being the friend he is, but also for his help on the model-implementation for the vimentin fits and the improvements of the code.

My deepest gratitude to everybody from the IRP, who made the time in Göttingen a memorizable time. Thank you all for the great discussions. Furthermore, I would like to thanks Christine Wilke-Feist, Eva Hetzel, Kerstin Pluschke, Sabine Balder and Michaela Ständer for the absolute wonderful job you did when it came to extending contracts, sick leaves, Dienstreiseanträgen or Reisekostenabrechnungen. To Jochen Herbst, Peter Luley, Susanne Hengst, Ulrike Rölleke, Kamila Sabagh and Jan Goeman my gratitude for all their help regarding vacuum pumps and chillers, clean-room assistance, noise cancelling issues, labstuff or IT problems.

A great thanks to all the members of the group.

I furthermore want thank Susanne Bauch, a very joyful and always helpful person. She did not 
only purify the vimentin which was used in this thesis and help me in the lab, but she also recommended a lot of 'must have been to' sights in Göttingen and the surrounding. I'm very grateful that during the 4 long years I always had Eleonora Perego at my side through this roller-coaster ride called PhD. Many thanks to Gerrit Brehm, who always had an open ear to all concerns I had. Without his work as a master student on the COC devices and the discussions, the microfluidics project would not have come that far. I would like to give my special thanks to Julia Kraxner, a wonderful person who brightened up my last three years. Thanks for all the helpful discussions about vimentin during our chocolate breaks. Special thanks to Anna Zelená, who was always able to make me laugh. For the help with Illustrator I am very thankful. Special thanks to Chiara Cassini, who was not only the best beamtime partner I had but who always had the right words and hugs to cheer me up. To Anna Schepers the best motivator, my deepest gratitude. She made me see the glitter and the Strassteinchen in my work. Thank you for all the helpful discussions. Many thanks to Johanna Forsting, who always had an open ear for me. She showed me the handling of vimentin and I am thankful for all the profitable discussions about my project. A special thanks to Jana Hanke for the discussions and the 3 o'clock tea times. Thanks to Clément Hémonnot and Oliva Saldanha for helping me getting started with my project. For the kind introduction to IFs and X-rays. Thanks to Clément for helping me getting started with Matlab and debugging my first codes. My gratitude to Martha Brennich for helping me understand the code for the micelle model better. I would furthermore like to thank Lotta Lorenz, Andrew Wittmeier, Sophie-Charlotte August, Jan-Philipp Burchert, Heidi Somsel and Anna Blob for all your help and beneficial discussions. I would like to thank, Julia, Chiara, Anna S., Anna Z., Eleonora, Heidi, Johanna, Jana, Michael and Janine for proof reading parts of my thesis. Thanks to Gerrit, Chiara, Andrew, Eleonora and Oliva for the help during the beamtimes.

Meinen Eltern, Renate und Werner, danke ich für die vielen Jahre der Unterstützung. Ohne ihre Hilfe wäre ich nie so weit gekommen. Auch einen besonderen Dank für die vielen Diskussionen und Anregungen zu meiner Arbeit. Meinem Bruder Michael und seiner Frau Janine, danke ich für die Diskussionen und die Unterstützung, besonders für die last-minute Hilfe mit Latex. Meinem Partner Christoph danke ich für alles was er mit mir in den letzten 4 Jahren durchgemacht hat. Dass er die Geduld mitgebracht hat, auch an einem Wochenende anstelle eines schönen Ausflugs mit mir in die Uni gekommen ist, damit ich meine Experimente machen konnte. Meinen Freunden danke ich besonders für die Unterstützung jeglicher Art, sowie die Nachsicht, wenn ich mal wieder einen Geburtstag verpasst habe. 